\title{
Efimov Physics: a review
}

\author{
Pascal Naidon ${ }^{1}$ and Shimpei Endo ${ }^{2}$ \\ ${ }^{1}$ RIKEN Nishina Centre, RIKEN, Wako, 351-0198 Japan. pascal@riken.jp \\ ${ }^{2}$ School of Physics and Astronomy, Monash University, Clayton, VIC, 3800, \\ Australia.shimpei.endo@monash.edu
}

Published on March 28 ${ }^{\text {th }}, 2017$ in Rep. Prog. Phys. 80, 056001, at the invitation of Professor Gordon Baym. Revised on October 3rd, 2022 (see list of changes at the end).

\begin{abstract}
This article reviews theoretical and experimental advances in Efimov physics, an array of quantum fewbody and many-body phenomena arising for particles interacting via short-range resonant interactions, that is based on the appearance of a scale-invariant three-body attraction theoretically discovered by Vitaly Efimov in 1970. This three-body effect was originally proposed to explain the binding of nuclei such as the triton and the Hoyle state of carbon-12, and later considered as a simple explanation for the existence of some halo nuclei. It was subsequently evidenced in trapped ultra-cold atomic clouds and in diffracted molecular beams of gaseous helium. These experiments revealed that the previously undetermined threebody parameter introduced in the Efimov theory to stabilise the three-body attraction typically scales with the range of atomic interactions. The few- and many-body consequences of the Efimov attraction have been since investigated theoretically, and are expected to be observed in a broader spectrum of physical systems.
\end{abstract}

\section{Contents}

\section{Introduction}

1 What is Efimov physics?

2 Why is it important? For which systems?

3 A short history of Efimov physics

\section{Three particles}

4 Three identical bosons

4.1 The Efimov universal theory . . . . . . . 7

4.2 Finite-range interactions . . . . . . . . 11

4.3 Other interactions . . . . . . . . . 13

4.3.1 Coulomb interactions . . . . . 13

4.3.2 Dipolar interactions . . . . . . . 14

4.3.3 Inverse-square interactions and generalised Efimov effect . . . . . 15
4.4 Relativistic case . . . . . . . . . . . 16

4.5 What is an Efimov state? . . . . . . 17

4.5.1 Energy............ . . 18

4.5.2 Structure ......... . . . 18

4.6 Observations in nuclear physics . . . . 19

4.6.1 The Hoyle state of carbon-12 . . 20

4.7 Observations with atoms . . . . . . . . 21

4.7.1 Helium-4 .......... . 21

4.7.2 ultra-cold atoms under Feshbach resonances . . . . . . . . 23

4.8 Prospects for observation in condensed matter ............. 25

4.8.1 Efimov states in quantum spin systems . . . . . . . . . 26

4.8.2 Universal few-body physics with excitons .......... 26

5 Three identical fermions 26

5.1 Resonant $p$-wave interactions . . . . . . 27

5.2 The super-Efimov effect . . . . . . . . 27

6 Multi-component systems 28 
6.1 Three distinguishable particles . . . . . 29

6.1.1 Three resonantly interacting pairs 29

6.1.2 Two resonantly interacting pairs 29

6.1.3 One resonantly interacting pair . 29

6.22 Identical particles +1 particle . . . . 30

6.2.1 2 bosons +1 particle with $\ell=0 \quad 31$

6.2.2 2 fermions +1 particle with $\ell=132$

6.2.3 Trimers with higher-angular momenta .......... 32

6.2.4 The Born-Oppenheimer picture . 32

6.2.5 Kartavtsev-Malykh universal trimers .......... 34

6.3 Particles with spin . . . . . . . . . 36

6.3.1 Rotationally invariant systems . 36

6.3.2 Polarised systems . . . . . . . . . 36

6.3.3 Spin-orbit interaction . . . . . 36

6.4 Observations in nuclear physics . . . . . 36

6.4.1 Triton ........... . . 37

6.4.2 Two-neutron halo nuclei . . . . . 40

6.5 Observations with atoms . . . . . . . . 42

6.5.1 Two-component trimers . . . . . 42

6.5.2 Three-component trimers ... . 43

\section{Dimensionality}

7 Situation in 1D and 2D

7.1 One dimension . . . . . . . . . . . . . 45

7.1.1 Identical bosons . . . . . . . . 45

7.1.2 2 particles +1 particle . . . . . 45

7.2 Two dimensions . . . . . . . . . . . . 46

7.2.1 Identical bosons . . . . . . . . 46

7.2.2 2 particles +1 particle . . . . . 46

8 Effects of confinement

8.1 From 3D to quasi-2D . . . . . . . . . . 47

9 Mixed dimensions $\quad 48$

9.1 The specificity of $D=3 \ldots \ldots$. . . . . 48

9.2 Interactions with three relative coordinates . . . . . . . . . . 5 50

9.3 Confinement-induced Efimov effect . . . 50

9.4 Stable Efimov trimers in bilayer or biwire geometries . . . . . . . . 50

9.5 Observations with ultra-cold atoms . . . 51

\section{The three-body parameter}

10 What is the three-body parameter? 52

10.1 In the zero-range theory . . . . . . . . 52

10.2 In systems with finite-range interactions 52

10.3 In systems with loss . . . . . . . . 52

11 What sets the three-body parameter? 53

11.1 First calculations . . . . . . . . . . . 53

11.2 Van der Waals universality . . . . . . . 54

11.2.1 Three identical bosons . . . . . . . 54

11.2.2 2 bosons +1 particle . . . . 58
11.3 Other types of short-range interactions . 59

11.3.1 Two-body correlation and effective range .......... 60

11.3.2 Deep-potential limits . . . . . . . . 61

11.3.3 Classes of universality . . . . . . 61

11.4 Coupled-channel interactions . . . . . . 61

11.4.1 Feshbach resonances ...... . . 61

11.4.2 Broad resonances . . . . . . . . 62

11.4.3 Narrow resonances . . . . . . . 62

11.4.4 Intermediate resonances . . . . . 63

11.4.5 Experimental observations . . . . 64

\section{More than three particles $\quad 65$}

12 Bosons $\quad 65$

12.1 Tetramers tied to Efimov trimers . . . 65

12.1.1 Four identical bosons . . . . . . 65

12.1.2 3 bosons +1 particle. . . . . . 66

12.2 Universal clusters . . . . . . . . . . . 67

12.2.1 Clusters below the ground-state trimer . . . . . . . . . 67

12.2.2 Universal $N$-body clusters . . . 68

12.3 Observation with atoms . . . . . . 68

13 Mass-Imbalanced Fermi mixtures $\quad 69$

13.12 fermions +2 fermions . . . . . . . . 69

13.23 fermions +1 particle . . . . . . . . 69

13.2.1 Four-body Efimov effect . . . . . 70

13.2.2 Universal four-body bound state 70

13.2.3 Five bodies and beyond . . . . . 70

\section{Many-body systems 71}

14 Many-body background 71

14.1 Efimov states in a Fermi sea . . . . . . 71

14.1.1 Two bosons and a fermion in a Fermi sea . . . . . . . . . 71

14.1.2 Three fermions in a Fermi sea . . 71

14.2 Efimov states in a condensate . . . . . . 72

14.2.1 Two impurities and a boson from a BEC . . . . . . . . . 72

14.2.2 One impurity and two bosons from a BEC . . . . . . . . 72

15 Many-body phases 72

15.1 Identical bosons. . . . . . . . . . . . . 73

15.1.1 Three-body contact in a Bose gas 73

15.1.2 The non-degenerate unitary

Bose gas . . . . . . . . . . 73

15.1.3 The Efimov liquid phase . . . . . 74

15.2 Trimer phases in Fermi mixtures . . . . 75

15.2.1 Three-component Fermi mixtures 75

15.2.2 Two-component Fermi mixtures $\quad 76$

VII Conclusion 


\section{Part I}

\section{Introduction}

\section{What is Efimov physics?}

In 1970, Vitaly Efimov found a remarkable effect in the quantum spectrum of three particles $[1,2]$. He considered particles interacting through short-range attractive interactions that are nearly resonant. By short range, one means interactions decaying faster than $1 / r^{3}$ where $r$ is the interparticle distance, and by nearly resonant, one means attractive interactions that can almost or just barely support a weakly two-body bound state. The fact that, in quantum mechanics, an attractive interaction may be too weak to bind two particles is due the quantum fluctuations of the kinetic energy (also known as the zero-point energy) that competes with the attractive interaction. When the interaction is just strong enough to cancel the repulsive effect of the kinetic energy, the interaction is said to be resonant because two particles scattering at low energy are very close to binding during their collision: they spend a long time together (they "resonate") before separating, which is characterised in scattering theory by an $s$-wave scattering length that is much larger than the range of the interactions.

Under these conditions, Vitaly Efimov found that an effective long-range three-body attraction arises, and this attraction may support an infinite family of three-body bound states (called Efimov states or Efimov trimers), in which the three particles are bound at larger and larger distances, beyond the range of the interactions. The Efimov effect, as it became known, is striking in several aspects:

Induced long-range interaction Even though the interactions are short-ranged, the three particles feel a long-range three-body attraction. This seemingly counter-intuitive situation can be explained by the fact that an effective interaction is mediated between two particles by the third particle moving back and forth between the two. It is thus possible for the three particles to feel their influence at distances much larger than the range of interactions, typically up to distances on the order of the scattering length.

Discrete scale invariance Right at the resonance, the scattering length is infinite and the effective attraction extends to infinite distances. Being of kinetic origin (the exchange of a particle between two others), the attraction scales like the kinetic energy of the particles and brings no characteristic length scale. As a result, the three-body system is scale invariant. Quantisation in this attractive potential gives an infinite series of bound states, the Efimov trimers, whose properties such as size and energy are related to each others' by a scale transformation with a universal scaling factor.
The energy spectrum, for instance, forms a geometric series with an accumulation at the zero energy threshold, corresponding to infinitely weakly bound states. This situation is referred to as the "discrete scale invariance" of Efimov states. Efimov states thus look like a infinite family of matryoshka, the Russian wooden dolls that can be nested inside each other. This image was originally given to describe renormalisation-group limit cycles [3, 4], which constitute a possibility among the general classification of renormalisation-group limits, originally proposed by Kenneth G. Wilson [5]. This possibility, which exhibits discrete scale invariance, is indeed realised in systems exhibiting the Efimov effect $[6]^{1}$.

Borromean binding When the interaction is not strong enough to support a two-body bound state, it may nonetheless support one, up to infinitely many, Efimov trimers. This possibility of binding $N$ particles, while the $N-1$ subsystems are unbound is called "Borromean" binding ${ }^{2}$. This denomination derives from the ancient symbol of intricated circles called "Borromean rings", which have been used, among others, by the Borromeo family in their coat of arms. Borromean rings are arranged in such a way that they cannot be separated, although cutting one of them sets the others free. They therefore constitute a classical example of Borromean binding. In this case, the binding is due to their specific topology. In the case of quantum particles, however, Borromean binding is possible even if the interparticle interaction is isotropic and does not enjoy such topological properties. Although it is counterintuitive from a classical point of view, it may be understood by considering that the number of degrees of freedom providing a zero-point kinetic energy scales like $N$ whereas the number of pairwise interactions scales like $N^{2}$, making the interactions win for sufficiently large $N$. Efimov trimers are an example of this phenomenon for $N=3$.

More difficult to interpret is the fact that when the interaction is strong enough to support a two-body bound state, further increasing the interaction reduces the binding of the three-body bound state with respect to that of the two-body bound state.

In recent years, it has been realised that the Efimov effect gives rise to a broad class of phenomena that have been referred to as Efimov physics. Consequences and extensions of the Efimov effect have indeed been found in systems of various kinds of particles, from three to many particles, with various kinds of interactions and in various mixtures of dimensions. The denomination

\footnotetext{
${ }^{1}$ Other examples of systems exhibiting the renormalisationgroup limit cycle are systems with $1 / r^{2}$ two-body interactions $[7,3]$ such as an electron scattering off an excited hydrogen atom. A more general discussion on discrete scale invariance is given in reference [8].

${ }^{2}$ Some authors $[9,10]$ reserve the term "Borromean" for $N=3$ and use the term "Brunnian" for larger $N$.
} 
"Efimov physics" is however not clearly defined and somewhat subjective. Even the notion of what constitutes an Efimov state has been debated and considerably extended by some authors - see section 4.5. In the strictest sense, "Efimov physics" designates physics that is a direct consequence of the occurence of the Efimov effect. More generally speaking, one may say that a system exhibits Efimov (or Efimov-like) physics when a three-or-more-body attraction emerges from shortrange interactions and possibly exhibits some kind of discrete scale invariance.

The purpose of this review is to cover the recent theoretical and experimental advances in Efimov physics, taken in its broadest sense.

\section{Why is it important? For which systems?}

Efimov physics is not only remarkable for its distinctive properties, it is also part of what is often referred to as the universality of low-energy physics. When a physical system with short-range interaction has a sufficiently low energy, its wave function is so delocalised that many microscopic details of the interactions become irrelevant, and most of its properties can be effectively described by a few parameters. Such physics is universal as it can be applied to many different systems, regardless of their microscopic details. Efimov physics is an example of such a situation, as it involves states in which the particles are on average at larger separations than the range of their interactions. For instance the discrete scale invariance of Efimov states is a universal feature that depends only on a few general properties such as the particles' masses and quantum statistics. As a result of this universality, Efimov physics applies to virtually any field of quantum physics, be it atomic and molecular physics [11, 12], nuclear physics [13], condensed matter [14] or even highenergy physics $[15,16]$. Interestingly, thanks to the formal connection between quantum theory and statistical physics, it may also apply to the thermal equilibrium of classical systems, such as three-stranded DNA [17].

The universality of Efimov physics does not mean that it occurs in any system. It means that any system meeting the conditions for its appearance exhibits the same universal features. These conditions turn out to be quite restrictive, which is why it has taken around forty years since the original theoretical prediction of the Efimov effect to obtain convincing experimental confirmations. Generally speaking, the Efimov effect requires resonant short-range interactions. Such interactions are rare, because they require a bound or virtual state to exist accidentally just below the scattering threshold of two particles. This situation turns out to be common in nuclear physics, but most nuclear particles obey Fermi statistics, and the Pauli exclusion between fermions overcomes the Efimov attraction in most cases, preventing the Efimov effect from occurring. On the other hand, bosonic particles or excitations are common in various fields of physics, but their interaction is rarely resonant. Nevertheless, there are now a significant number of physical systems where Efimov physics has been observed or is expected to be observed. In particular, with the advent of controllable Feshbach resonances in ultra-cold atomic gases it has become possible to fulfill at will the conditions for the occurence of Efimov physics, and study it extensively.

Since there have been many theoretical developments in Efimov physics recently, this review is organised from the theoretical point of view in terms of physical situations leading to Efimov or Efimov-like physics. For each situation, the current state of experimental observation in different fields of physics is presented. Although this choice of presentation requires the reader to read different sections to know about the experimental achievements in a particular field, it should give a comprehensive overview of what has, and what has not yet, been observed in Efimov physics. The sections are relatively independent, so that the reader can jump directly to the situation of their interest. As for the readers who desire to grasp the bare essentials of the Efimov effect, we have included a concise derivation of Efimov theory in section 4.1 and discussed the main features of Efimov states in section 4.5. In addition, we give in the following section a short history of the development of Efimov physics underlining the landmarks contributions.

\section{A short history of Efimov physics}

In 1970, Vitaly Efimov was working as a junior researcher at the Ioffe Institute in Leningrad, where he had completed his doctoral thesis four years earlier. Following the seminal work of Llewellyn H. Thomas in 1935 [18] and later works by G. V. Skorniakov and Karen A Ter-Martirosian [19], he was interested in the three-body problem in quantum mechanics to describe nuclear systems such as the triton (the nucleus of tritium, made of one proton and two neutrons). Thomas had shown that three particles with a symmetric wave function, unlike two particles, can be bound with arbitrarily large binding energy for sufficiently small range of the interparticle attractive force. This finding, referred to as the "Thomas collapse" or "fall to the centre" seemed somewhat peculiar, but allowed Thomas to estimate a lower bound for the range of nuclear forces from the measured energy of tritium, before it was confirmed by neutron-proton scattering experiments.

Using the hyper-spherical coordinates, Efimov found that when two of the particles can nearly bind, the three particles actually admit an infinite series of bound states of ever-increasing sizes, instead of just one as previously anticipated. This was due to an effective three-body attractive force, which gave a simple interpretation for the Thomas collapse. He published his 


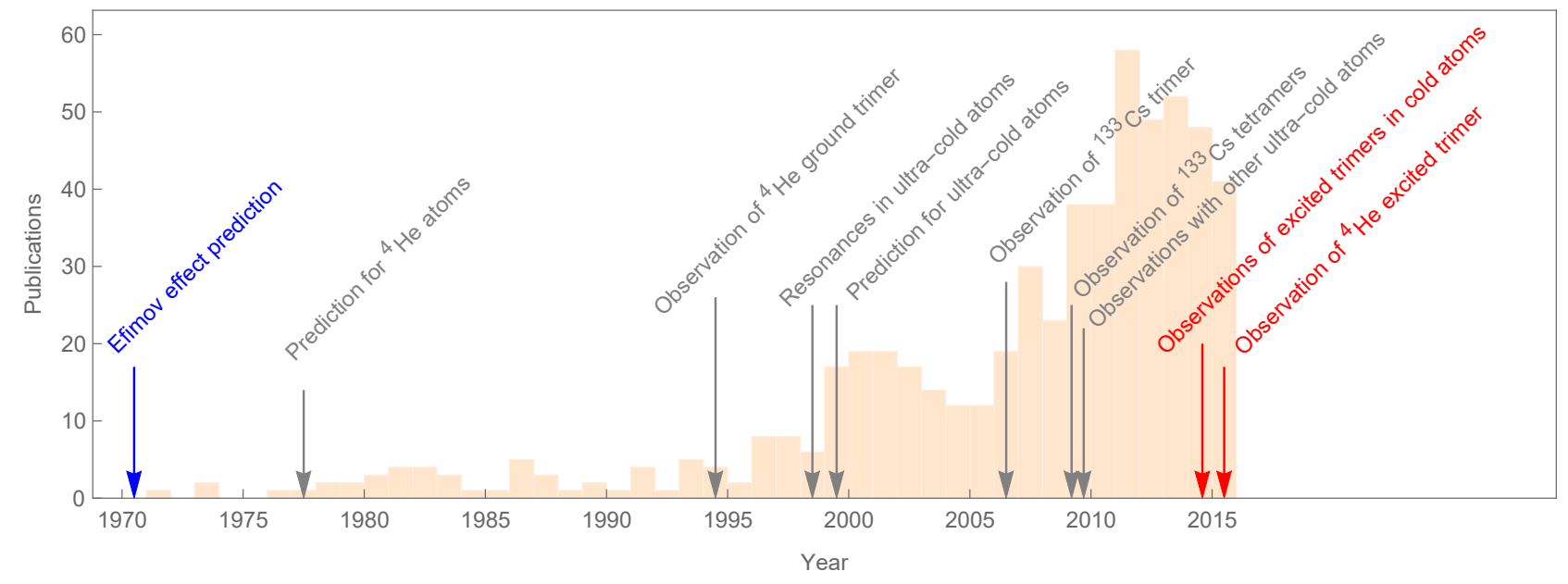

Figure 3.1: History of Efimov physics from the original theoretical prediction by Vitaly Efimov to the latest experimental observations, along with the number of related publications (source: Web of Knowledge).

result in both the Soviet literature $[1,20]$ and Western journals $[2,21]$ where it became known as the "Efimov effect". The first publication in English did not provide the derivation and the effect was thus met with scepticism. However, it prompted some theorists to look into the problem and soon after, the validity of Efimov's result was confirmed both analytically and numerically by R. D. Amado and J. V. Noble [22]. For a long time, however, the Efimov effect was regarded by many as a theoretical peculiarity of the formal three-body problem that would have little to virtually no observable consequences on real physical systems. On the other hand, some people took the effect seriously and tried to find physical systems where it could be observed.

Vitaly Efimov proposed in his original papers that the Efimov effect could describe nuclear systems such as the triton and the famous Hoyle state of carbon-12. Subsequently, it was suggested that the Efimov effect may be revealed in some hypernuclei by T. K. Lim in 1986 [23], and in halo nuclei by Dmitri V. Fedorov, Aksel S. Jensen, and Karsten Riisager in 1994 [24]. The proposed nuclear systems indeed feature resonant twobody subsystems, which is a requirement for the Efimov effect to occur. The closer to resonance the twobody subsystems are, the larger the number of threebody bound states. However, having more than one three-body bound state requires a very close tuning near the resonance, something that happens only accidentally in nature. As a result, the proposed nuclear systems allow only one three-body bound state to exist, and do not reveal the infinity of other states predicted by the Efimov theory closer to resonance. Moreover, it is difficult to show that such a single three-body bound state originates from the Efimov effect for two reasons. First, Efimov's theory relies on an unknown three-body parameter to describe the three-body states, and is thus not quite predictive for the properties of a single three-body state, whereas it makes definite and universal predictions (independent of the three-body pa- rameter) for the relative properties of two three-body states. Second, the first three-body state is the smallest of the Efimov series and is significantly affected by the details of the interparticle forces, to the point that it is debatable to call it Efimov state. Because of these ambiguities, and despite the experimental observations of the proposed nuclear systems, it has been difficult to prove or disprove that they are indeed Efimov states.

To obtain better experimental evidence of the Efimov effect, researchers turned to other kinds of particles for which the two-body resonance condition could be more closely fulfilled. Seven years after Efimov's theory, T. K. Lim already pointed out the particular case of helium-4 atoms [25], whose interatomic interaction is close enough to resonance to admit two threebody bound states, as was checked subsequently by many few-body theorists. This prompted a decadelong experimental search for these two helium- 4 trimer states by the group of Jan Peter Toennies in Göttingen, by analysing diffracted beams of helium-4 clusters [26, 27]. While the ground-state trimer could be observed, the excited trimer state, which is regarded as a true Efimov trimer and an evidence of the Efimov effect, could not be observed.

The breakthrough that established Efimov physics came from the field of ultra-cold atoms. In the 1990s, it was predicted [28] and demonstrated experimentally $[29,30]$ that the interactions between atoms could be controlled and brought to resonance by applying a magnetic field. This led to the proposal by Brett D. Esry, Chris H. Greene, and James P. Burke Jr [31] to observe the signatures of Efimov states in such systems. Such experimental signature of a three-body state near the two-body resonance of caesium-133 atoms was obtained in 2002 in the group of Hanns-Christoph Nägerl and Rudolf Grimm in Innsbruck, and after careful analysis reported in 2006 [32]. Although it revealed only one trimer, as in the nuclear systems or the previous experiments on helium, its Efimovian nature ap- 
peared more convincing due to its Borromean nature (the trimer exists in a region where two-body subsystems are known to be unbound). This landmark experiment opened the way for a systematic investigation of Efimov physics, because the interaction could now be controlled. This led to many similar experimental results from various laboratories around the world using other species of ultra-cold atoms (in particular during the year 2009) as well as an intense theoretical activity to understand and explore various aspects of Efimov physics in ultra-cold atoms. In the same year, universal four-body bound states tied to Efimov states were evidenced in the caesium experiment in Innsbruck by Francesca Ferlaino and co-workers [33], just after being predicted by theorists $[34,35]$. The year 2009 culminated with the ITAMP workshop in Rome entitled "Efimov 2009", where the wealth of new experimental and theoretical results was presented.

With the accumulation of experimental results in ultra-cold atoms, the theoretically unknown threebody parameter of the Efimov theory could be obtained from experimental measurements for many different Efimov states. In particular, it could be compared for different two-body resonances in the lithium-7 experiments by the group of Lev Khaykovich at Bar-Ilan University [36], and in the caesium experiments by the group of Innsbruck [37]. To everyone's surprise, the three-body parameter was found to be nearly the same for all the resonances of a given atomic species. It even appeared to be universally correlated to the van der Waals length of the atoms, while it was thought to depend on many other microscopic details. This so-called "van der Waals universality" of the three-body parameter was later explained by theoretical works [38, 39], which showed that a sudden deformation of the trimer configuration prevents the three atoms from reaching separations smaller than the van der Waals length, making the trimers insensitive to more microscopic features of the interatomic interaction.

While different measurements of three-body recombination and atom-dimer relaxation provided several experimental points in the three-body spectrum confirming the "scenario" obtained by Vitaly Efimov, the most striking aspect of this scenario, namely the discrete scale invariance leading to the geometric series of three-body bound states was not confirmed clearly since the experiments did not reveal consecutive threebody bound states. Observing consecutive Efimov states is an experimental challenge since each new state is by definition much larger in size with a much weaker binding energy. This endeavour was ultimately successful in 2014, when the experimenters in Innsbruck managed to observe a second Efimov state of caesium atoms by pushing the limits of their experiment [40], while the groups of Cheng Chin at the University of Chicago [41] and Matthias Weidemüller at the University of Heidelberg [42] independently observed up to three Efimov states of two caesium and one lithium atoms, whose en- ergy levels were predicted to be closer to each other due to the large mass imbalance between these two atomic species. The same year, outside the ultra-cold atom community, the group of Reinhard Dörner in Frankfurt could finally observe the long-sought second trimer of helium- 4 by the Coulomb explosion imaging technique, a result published the following year [12]. Not only this brought further experimental confirmation of the Efimov effect, it also provided the first spatial imaging of an Efimov state. One may say that the year 2014 concluded a 44-year-long search for a full confirmation of the Efimov effect. The history of this search is summarised in figure 3.1 where landmark contributions are indicated. 


\section{Part II}

\section{Three particles}

\section{Three identical bosons}

The simplest situation for which Efimov physics occurs corresponds to three identical bosons interacting via resonant short-range interactions. In section 4.1, we briefly present the corresponding theory originally proposed by Vitaly Efimov and its various extensions in sections 4.2-4.4. In section 4.5, we look into the question of what constitutes an Efimov state, before reviewing in sections 4.6-4.8 the experimental observations and prospects for observations of bosonic Efimov states in nuclear, atomic, and condensed matter systems.

\subsection{The Efimov universal theory}

We consider identical bosonic particles of mass $m$, with no internal degree of freedom, interacting via shortrange two-body (and possibly three-body) interactions. Here, short-range interactions means that the interaction potentials decay faster than $1 / r^{3}$, where $r$ is the separation between two particles. In this situation, there exists a separation $b$, called the range of the interaction, beyond which the relative motion of two particles is almost free. It is in this asymptotically free region where the particles' energy is purely kinetic that the Efimov effect takes its roots, and that is why it is universal.

Although the relative motion of two particles is free in this region, each angular partial wave of the wave function $\psi(\vec{r})$ describing the two-body relative motion has a phase shift $\delta_{\ell}$ with respect to the non-interacting wave function, as a result of the particles interacting at shorter separation. Namely, in the partial-wave expansion of $\psi(\vec{r})$,

$$
\psi(\vec{r})=\psi(r, \theta, \phi)=\sum_{\ell=0}^{\infty} \frac{f_{\ell}(r)}{r} P_{\ell}(\cos \theta),
$$

where $P_{\ell}$ are the Legendre polynomials, the partial wave component $f_{\ell}(r)$ has the form,

$f_{\ell}(r)= \begin{cases}\text { complicated } & \text { for } r \lesssim b \text { (interaction) } \\ \propto \sin \left(k r-\ell \frac{\pi}{2}+\delta_{\ell}\right) & \text { for } r \gg b \text { (free region) }\end{cases}$

where $k$ is the relative wave number between the two particles. In the absence of interaction, the phase shift $\delta_{\ell}=0$ (no scattering occurs). On the opposite, the strongest dephasing the interaction can induce is $\delta_{\ell}=$ $\pi / 2(\operatorname{modulo} \pi)$, in which case the interaction is said to be resonant in that partial wave.

Efimov physics arises when the two-body interaction is near-resonant in the $s$-wave partial wave $(\ell=0)$, which means that the phase shift $\delta_{0}$ of the $s$ wave is close to $\pi / 2$ (modulo $\pi)$.
The scattering length It is well-known from scattering theory [43] that at low scattering energy $\left(k \ll b^{-1}\right)$, only the $s$ wave is scattered, i.e. has a non-zero phase shift. Moreover, the phase shift can be written as

$$
\delta_{0} \sim-\arctan (k a) \quad \text { for } k \ll b^{-1},
$$

where $a$ defines the scattering length ${ }^{3}$. Therefore, for the two-body interaction to be resonant at low energy, the scattering length $a$ has to be much larger than $b$ :

$$
|a| \gg b
$$

In particular, the limit $a \rightarrow \pm \infty$ is sometimes called the unitary limit or unitarity, because in this limit the factor $\sin ^{2} \delta_{0}$ in the expression of the scattering cross section $\sigma=\frac{4 \pi}{k^{2}} \sin ^{2} \delta_{0}$, approaches its maximal value $\sin ^{2} \delta_{0}=1$. This maximum of the scattering cross section is the consequence of a fundamental property of quantum mechanics, the unitarity of the S-matrix. It can be reached precisely for resonant interactions.

Near unitarity, the scattering length $a$ is the only parameter that controls the physics of two particles at low energy, either positive or negative: it determines the cross section for scattering states (positive energy), and the binding energy of a weakly bound state below the break-up threshold (negative energy). This bound state, also called dimer, exists only for a positive scattering length and its binding energy is close to

$$
\frac{\hbar^{2}}{m a^{2}}
$$

where $m$ is the mass of the particles and $\hbar$ is the reduced Planck constant. The resonance of the interaction is therefore related to the appearance of the twobody bound state from below the scattering threshold exactly at the unitary limit $a \rightarrow \pm \infty$. It is represented by a black line in figure 4.2 .

Zero-range theory Short-range near-resonant interactions at low energy constitute a limit that can be treated by the zero-range theory. This theory assumes that the short-range region where the interaction directly affects the wave function can be neglected and only the asymptotically free region that is parameterised by the scattering length is relevant. This amounts to saying that the range $b$ of the interaction is vanishingly small compared to the scattering length $a$ or wave length $k^{-1}$ of the particles. This can be implemented in various ways.

A first way is to consider a simple interaction potential with a finite range $b$, calculate observables, and take the limit $b \rightarrow 0$ for a fixed scattering length $a$. Another way is to consider a zero-range pseudopotential, such as a contact interaction represented by a Dirac delta function potential, sometimes referred to

\footnotetext{
${ }^{3}$ Despite its name, the scattering length can be positive or negative.
} 
as a "Fermi pseudopotential" [44]. This introduces ultraviolet divergences in exact calculations which need to be renormalised to obtain observables [45]. Renormalisation can be implemented by introducing a cutoff in momentum space $[46,47,6]$, or regularising the delta function using the Lee-Huang-Yang pseudopotential [48]:

$$
\hat{V}(r)=\frac{4 \pi \hbar^{2} a}{m} \delta^{3}(\vec{r}) \frac{\partial}{\partial r}(r \cdot)
$$

Yet another way is to consider the system as free (no interaction) and impose the so-called Bethe-Peierls boundary condition [49] on the many-particle wave function $\Psi$ when any two particles separated by $r$ come in contact:

$$
-\frac{1}{r \Psi} \frac{\partial}{\partial r}(r \Psi) \underset{r \rightarrow 0}{\longrightarrow} \frac{1}{a}
$$

The essence of all these methods is to correctly reproduce the form of the two-body wave function in the region $b \ll r \ll k^{-1}$,

$$
\psi(\vec{r}) \propto \frac{1}{r}-\frac{1}{a},
$$

which can be obtained from equations (4.1), (4.2) and (4.3). The zero-range methods make the simplification that this form remains true down to $r=0$, although this is unphysical for $r \lesssim b$.

Such zero-range methods can be directly implemented in the Schrödinger equation describing the three-boson system [50], or alternative formalisms such as integral equations [19], functional renormalisation equations [51, 52], and effective field theory [53, 6]. Here, we will make use of the Schrödinger equation along with the Bethe-Peierls boundary condition (4.7).

We should note that Vitaly Efimov's original derivation [1] did not invoke explicitly a zero-range interaction, but instead considered equation (4.8), i.e. the effect of the resonant interaction outside its range $b$, without requiring $b \rightarrow 0$. As we shall see, the zerorange theory for three particles is in fact ill-defined. The more physical approach of Efimov avoids this difficulty and naturally introduces the three-body parameter. It should thus be referred to as a universal theory, instead of a zero-range theory. Nevertheless, it is essentially equivalent to the zero-range theory cured by a three-body boundary condition. For the sake of simplicity, we will take this path, which formally follows very closely Efimov's original derivation.

Derivation of the Efimov attraction For three bosons located at $\vec{x}_{1}, \vec{x}_{2}$ and $\vec{x}_{3}$, one can eliminate the centre of mass, and the system can be described by two vectors, called Jacobi coordinates:

$$
\begin{aligned}
\vec{r}_{i j} & =\vec{x}_{j}-\vec{x}_{i} \\
\vec{\rho}_{i j, k} & =\frac{2}{\sqrt{3}}\left(\vec{x}_{k}-\frac{\vec{x}_{i}+\vec{x}_{j}}{2}\right)
\end{aligned}
$$

where $(i, j, k)$ are to be chosen among $(1,2,3)$. There are thus three possible Jacobi coordinate sets, shown in figure 4.1, which are related as follows:

$$
\begin{aligned}
\vec{r}_{23} & =-\frac{1}{2} \vec{r}_{12}+\frac{\sqrt{3}}{2} \vec{\rho}_{12,3} \\
\vec{\rho}_{23,1} & =-\frac{\sqrt{3}}{2} \vec{r}_{12}-\frac{1}{2} \vec{\rho}_{12,3} \\
\vec{r}_{31} & =-\frac{1}{2} \vec{r}_{12}-\frac{\sqrt{3}}{2} \vec{\rho}_{12,3} \\
\vec{\rho}_{31,2} & =\frac{\sqrt{3}}{2} \vec{r}_{12}-\frac{1}{2} \vec{\rho}_{12,3}
\end{aligned}
$$

Choosing one set of Jacobi coordinates, the timeindependent three-body wave function satisfies the free Schrödinger equation at total energy $E=\hbar^{2} k^{2} / m$ :

$$
\left(-\nabla_{r_{12}}^{2}-\nabla_{\rho_{12,3}}^{2}-k^{2}\right) \Psi=0
$$

along with the Bethe-Peierls boundary condition (4.7) for all pairs of bosons. Because of the bosonic exchange symmetry, the wave function $\Psi$ can be decomposed as follows:

$$
\Psi=\chi\left(\vec{r}_{12}, \vec{\rho}_{12,3}\right)+\chi\left(\vec{r}_{23}, \vec{\rho}_{23,1}\right)+\chi\left(\vec{r}_{31}, \vec{\rho}_{31,2}\right)
$$

where the function $\chi$ (known as Faddeev component $[54,50])$ satisfies the equation:

$$
\left(-\nabla_{r}^{2}-\nabla_{\rho}^{2}-k^{2}\right) \chi(\vec{r}, \vec{\rho})=0
$$

Applying the Bethe-Peierls boundary condition (4.7) for the pair $(1,2)$ to equation $(4.16)$, one obtains:

$$
\begin{aligned}
& {\left[\frac{\partial}{\partial r}(r \chi(\vec{r}, \vec{\rho}))\right]_{r \rightarrow 0}+\chi\left(\frac{\sqrt{3}}{2} \vec{\rho},-\frac{1}{2} \vec{\rho}\right)+\chi\left(-\frac{\sqrt{3}}{2} \vec{\rho},-\frac{1}{2} \vec{\rho}\right) } \\
= & {\left[-\frac{r}{a}\left(\chi(\vec{r}, \vec{\rho})+\chi\left(\frac{\sqrt{3}}{2} \vec{\rho},-\frac{1}{2} \vec{\rho}\right)+\chi\left(-\frac{\sqrt{3}}{2} \vec{\rho},-\frac{1}{2} \vec{\rho}\right)\right)\right]_{r \rightarrow 0} }
\end{aligned}
$$

where $\vec{r} \equiv \vec{r}_{12}$ and $\vec{\rho} \equiv \vec{\rho}_{12,3}$. From the bosonic exchange symmetry, the same equation is obtained by applying the Bethe-Peierls boundary condition for the other two pairs. In the right-hand side of equation (4.18), only the first term remains when $r \rightarrow 0$, because $\chi(\vec{r}, \vec{\rho})$ diverges for $r \rightarrow 0$ but is finite elsewhere. The function $\chi$ can be expanded in partial waves, which can be shown to be independent in the zero-range theory. The Efimov effect for bosons occurs in the partial-wave channel with total angular momentum $L=0$. In this channel, $\chi$ is independent of the directions of $\vec{r}$ and $\vec{\rho}$ and can be written as

$$
\chi(\vec{r}, \vec{\rho})=\frac{\chi_{0}(r, \rho)}{r \rho} .
$$

$\chi_{0}$ is finite for $r \rightarrow 0$, consistent with the divergence of $\chi$, but must satisfy:

$$
\chi_{0}(r, \rho) \underset{\rho \rightarrow 0}{\longrightarrow} 0
$$



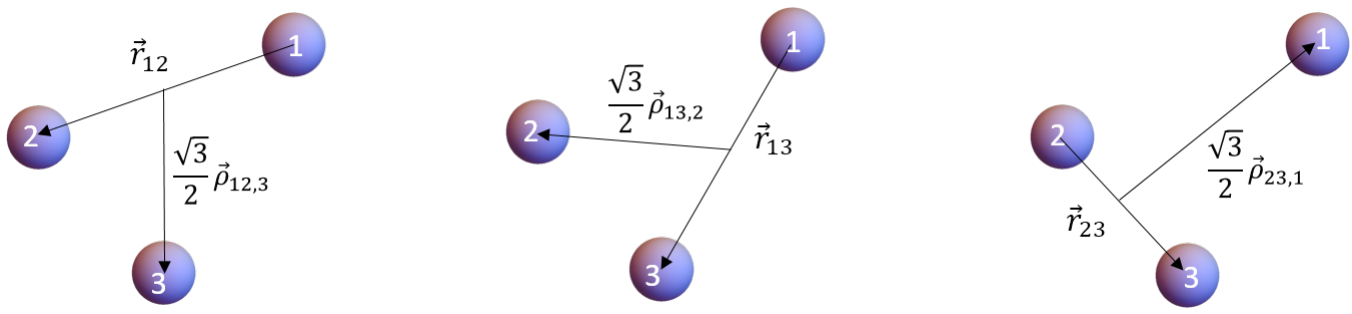

Figure 4.1: The three sets of Jacobi coordinates describing the relative positions of three identical particles.

to keep $\chi$ finite in this limit. Inserting equation (4.19) into equations (4.17) and (4.18) yields the equation

$$
\left(-\frac{\partial^{2}}{\partial r^{2}}-\frac{\partial^{2}}{\partial \rho^{2}}-k^{2}\right) \chi_{0}(r, \rho)=0
$$

and the boundary condition for $r \rightarrow 0$ :

$$
\left[\frac{\partial}{\partial r}\left(\chi_{0}(r, \rho)\right)\right]_{r \rightarrow 0}+2 \frac{1}{\frac{\sqrt{3}}{4} \rho} \chi_{0}\left(\frac{\sqrt{3}}{2} \rho, \frac{1}{2} \rho\right)=-\frac{1}{a} \chi_{0}(0, \rho)
$$

One can finally perform a transformation of the coordinates $(r, \rho)$ to the polar coordinates $(R, \alpha)$ known as hyper-spherical coordinates $[55,50]$ :

$$
\begin{aligned}
& r=R \sin \alpha \\
& \rho=R \cos \alpha
\end{aligned}
$$

where $R$ is the hyper-radius satisfying

$$
R^{2}=r^{2}+\rho^{2}=\frac{2}{3}\left(r_{12}^{2}+r_{23}^{2}+r_{31}^{2}\right)
$$

and $\alpha$ is the Delves hyper-angle. In these coordinates, one obtains the equation:

$$
\left(-\frac{\partial^{2}}{\partial R^{2}}-\frac{1}{R} \frac{\partial}{\partial R}-\frac{1}{R^{2}} \frac{\partial^{2}}{\partial \alpha^{2}}-k^{2}\right) \chi_{0}(R, \alpha)=0
$$

with the boundary condition for $\alpha \rightarrow 0$ :

$$
\left[\frac{\partial}{\partial \alpha}\left(\chi_{0}(R, \alpha)\right)\right]_{\alpha \rightarrow 0}+\frac{8}{\sqrt{3}} \chi_{0}\left(R, \frac{\pi}{3}\right)=-\frac{R}{a} \chi_{0}(R, 0)
$$

The problem then becomes separable in $R$ and $\alpha$, for the case $a \rightarrow \pm \infty$ corresponding to the unitary limit. Indeed, in this limit the right-hand side of equation (4.26) vanishes and one is left with a boundary condition at $\alpha=0$ that is independent of $R$. On the other hand, the other boundary condition (4.20) corresponds to $\chi_{0}\left(R, \frac{\pi}{2}\right)=0$, which is a boundary condition at $\alpha=\frac{\pi}{2}$ that is also independent of $R$. One can thus find a solution of equation (4.25) in the form:

$$
\chi_{0}(R, \alpha)=F(R) \phi(\alpha)
$$

where $\phi$ satisfies $-\frac{d^{2}}{d \alpha^{2}} \phi(\alpha)=s_{n}^{2} \phi(\alpha)$ with the boundary conditions at $\alpha=0$ and $\alpha=\pi / 2$. This gives the following solutions:

$$
\phi_{n}(\alpha)=\sin \left(s_{n}\left(\frac{\pi}{2}-\alpha\right)\right)
$$

where $s_{n}$ is a solution of the equation:

$$
-s_{n} \cos \left(s_{n} \frac{\pi}{2}\right)+\frac{8}{\sqrt{3}} \sin \left(s_{n} \frac{\pi}{6}\right)=0 .
$$

Each solution labelled by $n$ constitutes a channel for the hyper-radial motion. That is to say, for each solution $\phi_{n}$ there is a corresponding hyper-radial function $F_{n}(R)$ such that $F_{n}(R) \phi_{n}(\alpha)$ is a solution of equation (4.25). It satisfies the equation:

$$
\left(-\frac{\partial^{2}}{\partial R^{2}}-\frac{1}{R} \frac{\partial}{\partial R}+\frac{s_{n}^{2}}{R^{2}}-k^{2}\right) F_{n}(R)=0
$$

which can be written as a one-dimensional Schrödinger equation:

$$
\left(-\frac{\partial^{2}}{\partial R^{2}}+V_{n}(R)-k^{2}\right) \sqrt{R} F_{n}(R)=0
$$

with the hyper-radial potential,

$$
V_{n}(R)=\frac{s_{n}^{2}-1 / 4}{R^{2}}
$$

All solutions of equation (4.29) are real, except one denoted as $s_{0} \approx \pm 1.00624 i$ which is purely imaginary. As a result, the effective $\propto R^{-2}$ potential in equation (4.30) is attractive for the channel $n=0$. This is in contrast with the non-interacting three-body problem, where the boundary condition (4.26) is replaced by $\chi_{0}(R, \alpha) \underset{r \rightarrow 0}{\longrightarrow} 0$, leading to equation (4.28) with eigenvalues $s_{n}=2(n+1)$ that are all real. In this case, the effective $\propto R^{-2}$ potential of equation (4.32) is repulsive for all $n$. This repulsion is interpreted as a generalised centrifugal barrier due to the free motion of deformation of the three-body system. In the interacting problem at unitarity however, the channel $n=0$ leads to an effective three-body attraction

$$
V_{0}(R)=-\frac{\left|s_{0}\right|^{2}+1 / 4}{R^{2}} \text {. }
$$

This unexpected attraction is the basis for Efimov physics and is referred to as the Efimov attraction. It can be interpreted as the result of a mediated attraction between two particles by exchange of the third particle. 
The existence of this attraction shows that the zerorange theory for three bosons is not well defined. Indeed, equation (4.30) for $n=0$ is a Schrödinger equation for an attractive $1 / R^{2}$ potential, which is scale invariant since a $\propto 1 / R^{2}$ potential scales as the kinetic energy $\propto d^{2} / d R^{2}$ under a scaling transformation $R \rightarrow \lambda R$. It is known that such an equation admits a solution at any energy, and its spectrum is therefore not bounded from below $[56,57]$. Indeed, if the equation admits a solution at energy $E<0$, making the scaling transformation $R \rightarrow \lambda R$ with an arbitrary scaling factor $\lambda$ gives another solution at energy $\lambda^{2} E<0$. This means that under the Efimov attraction the threeboson system collapses on itself, a phenomenon discovered long ago by Llewellyn H. Thomas [18] and referred to as the "Thomas collapse" or "fall of the particles to the centre". The same problem was found [58] in the formulation of the zero-range theory for three particles by an integral equation, known as the Skorniakov and Ter-Martorisian equation [19]. This is of course a shortcoming of the zero-range theory, since the finite-range effects of the interaction can no longer be neglected when the distance between the three bosons becomes comparable with the finite range of interactions ${ }^{4}$.

A practical solution to this problem, originally suggested by Vladimir N. Gribov and demonstrated by G. S. Danilov [59], consists in imposing a condition on the solutions of the three-body equation, or a momentum cut-off on the equation [60], in order to reproduce a known three-body observable, such as a three-body bound state energy or particle-dimer scattering property. For instance, fixing the triton energy to the observed value, and solving the three-body equation with that condition enables the prediction of the neutrondeuteron scattering length [61].

In Vitaly Efimov's formulation of the three-body problem in terms of equation (4.30), a similar procedure can be achieved by imposing a boundary condition below some arbitrarily small hyper-radius $R_{0}$. Thus, in addition to the Bethe-Peierls two-body boundary condition (4.7), the three-body problem in the zero-range theory requires an extra three-body boundary condition. This boundary condition can be implemented in various ways, for example setting a hard wall at the hyper-radius $R_{0}$ where $F_{0}$ has to vanish, or imposing the value of the logarithmic derivative of $F_{0}$ at $R_{0}$, by analogy to the Bethe-Peierls condition (4.7). Note that these two implementations are not strictly equivalent: a hard wall prevents the fall to the centre and sets a ground-state energy, whereas a logarithmic derivative condition only makes the spectrum discrete but still unbounded from below (states below the physically relevant energy are therefore unphysical features). In any case, both implementations introduce a new length scale in the problem, which is referred to as the three-body parameter. It is this parameter that

\footnotetext{
${ }^{4}$ Throughout this article and much of the cited literature, the expression "finite range" means a range that is not zero.
}

fixes the three-body observables. The necessity to introduce this parameter may be regarded as a quantum anomaly in the scaling symmetry of the system [62]. Physically, the three-body parameter encapsulates the effects of the two-body (and possibly three-body) interactions at short distance.

To see how the three-body parameter arises, let us consider the solutions of equation (4.30) for $n=0$. Near the small hyper-radius $R_{0}$, any solution with sufficiently small energy $\left|k^{2}\right| \ll\left|s_{0}\right|^{2} / R_{0}^{2}$ is of the form:

$$
F_{0}(R)=\alpha R^{i\left|s_{0}\right|}+\beta R^{-i\left|s_{0}\right|} \quad \text { for } R \gtrsim R_{0}
$$

Imposing a boundary condition at $R_{0}$ imposes a specific ratio $\beta / \alpha$. From dimensional analysis, this ratio has units of inverse length $\Lambda$ to the power $-2 i\left|s_{0}\right|$. Thus, we can write $F_{0}(R)=\alpha\left(R^{i\left|s_{0}\right|}+\Lambda^{-2 i\left|s_{0}\right|} R^{-i\left|s_{0}\right|}\right)$, which can be further expressed as

$$
F_{0}(R) \underset{R \gtrsim R_{0}}{\propto} e^{i\left|s_{0}\right| \ln \Lambda R}+e^{-i\left|s_{0}\right| \ln \Lambda R}=\cos \left(\left|s_{0}\right| \ln \Lambda R\right) .
$$

The three-body wave function therefore shows logperiodic ocillations in the hyper-radius, and the phase of these oscillations

$$
\Phi=\left|s_{0}\right| \ln \left(\Lambda / \Lambda_{0}\right)
$$

is given by the new scale $\Lambda$ (expressed in some previously fixed unit $\Lambda_{0}$ ), which is a possible representation of the three-body parameter.

One of the fundamentally new findings of Vitaly Efimov is that the three-body problem with the threebody boundary condition does not only yield just one three-body bound state, as previously thought, but infinitely many bound states. This is a simple consequence of the effective attractive $1 / R^{2}$ potential in equation (4.30). Indeed, although the boundary condition (4.35) breaks the scale invariance of the system under arbitrary scale transformations, one can easily check that equation (4.35) is still invariant under a discrete set of scale transformations $R \rightarrow \lambda_{0}^{n} R$, with scaling factors that are integral powers of $\lambda_{0}=e^{\pi /\left|s_{0}\right|} \approx$ 22.7. Thus, if the boundary condition gives a solution at some energy $E<0$, it also gives solutions with energies $E / \lambda_{0}^{2 n}<0$. There is therefore an infinite number of bound states, forming a geometric series of energies accumulating at zero energy, with scaling factor $\lambda_{0}^{2} \approx 515$. This situation is referred to as the discrete scale invariance.

Remarkably, Vitaly Efimov has shown that this discrete scale invariance not only holds at unitarity $(a \rightarrow$ $\pm \infty$ ) but also at finite scattering length $a$, when one considers the spectrum in the polar coordinates $(h, \xi)$ of the inverse scattering length $a^{-1}$ and the wave number $\kappa=E \sqrt{m /\left(\hbar^{2}|E|\right)}$ :

$$
\begin{aligned}
a^{-1} & =h \cos \xi \\
\kappa & =h \sin \xi
\end{aligned}
$$




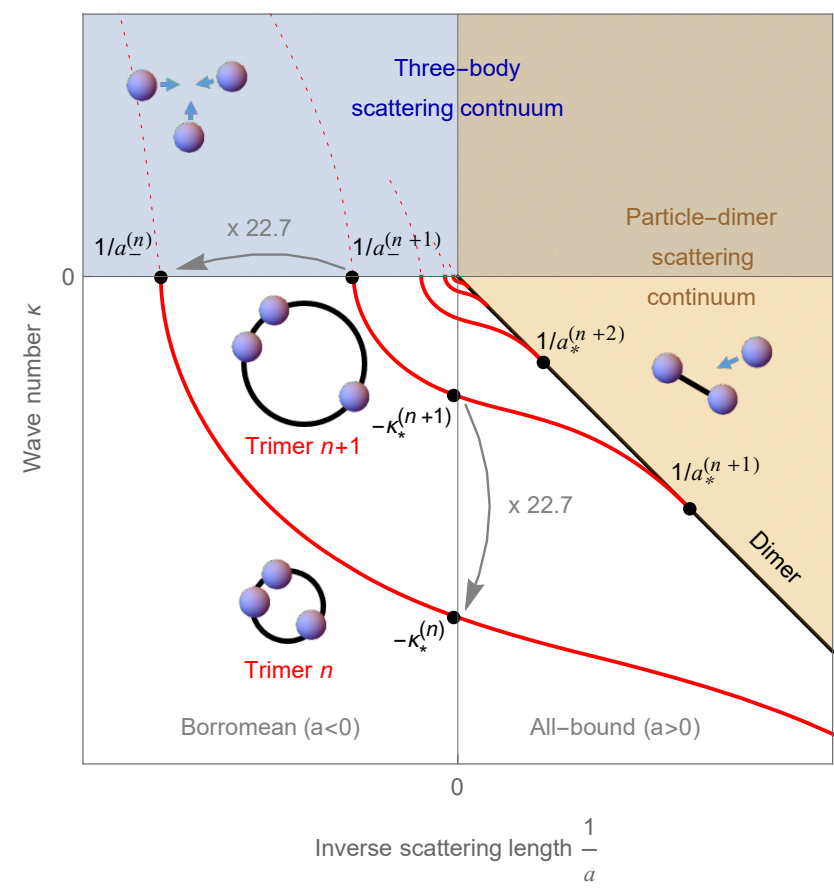

Figure 4.2: Schematic representation of the so-called "Efimov plot" or "Efimov scenario" showing the discrete scale invariance of the three-body spectrum for identical bosons in the zero-range theory. The wave number $\kappa=E \sqrt{m /\left(\hbar^{2}|E|\right)}$ associated with the energy $E$ of the dimer (black) and trimers (red) is plotted against the inverse scattering length $1 / a$. The blue and orange filled regions represent the three-body scattering continuum and the particle-dimer scattering continuum, respectively. Note that these continua overlap for $1 / a>0$ and $E>0$. Special values of $\kappa$ and $1 / a$ are indicated by the dots: a trimer appears from the three-body scattering threshold at $1 / a_{-}$, has a binding wave number $\kappa_{*}$ at unitarity, and disappears below the particle-dimer scattering threshold at $1 / a_{*}$. Trimer resonances in the three-body continuum are indicated by dotted curves. The discrete scale invariance of the spectrum is indicated by the grey arrows showing the universal scaling ratio between consecutive levels. For clarity, the value of the strength $s_{0}$ has been artificially set to 3 , instead of 1.00624 , thus reducing the spacings between the trimer levels to make them more visible.

The spectrum along a line at a fixed angle $\xi$ has the discrete scale invariance with the scaling factor $\lambda_{0}=e^{\pi /\left|s_{0}\right|} \approx 22.7$. This property can be checked by scaling $k, a^{-1}$, and $R^{-1}$ by $\lambda_{0}$ in equations (4.254.26). As a result, all the three-body bound states show the same trajectory in the $\left(a^{-1}, \kappa\right)$ plane up to a scale transformation, as shown in figure 4.2. The infinite series of bound-states energies $E^{(n)}$ can therefore be described by the discrete scaling of a single universal function $\Delta(\xi) \equiv 2\left|s_{0}\right| \ln h(\xi)$ through the formula

$$
\left|E^{(n)}\right|+\frac{\hbar^{2}}{m a^{2}}=\frac{\hbar^{2} \kappa_{*}^{2}}{m} e^{-2 \pi n /\left|s_{0}\right|} e^{\Delta(\xi) /\left|s_{0}\right|},
$$

where $n$ is an integer labelling the states, and $\kappa_{*}$ is the binding wave number at unitarity of the state $n=0$. The value of $\kappa_{*}$ is set by the three-body boundary condition, so that it may be regarded as a representation of the three-body parameter. A change in the value of $\kappa_{*}$ simply scales the curves in figure 4.2 inwards or outwards from the accumulation point $\left(a^{-1}=0, E=0\right)$.

The universal function $\Delta(\xi)$ has been determined numerically and approximated by analytical expressions in reference [63]. This numerical approximation has inaccuracies on the order of $3 \%$ for $\xi$ close to $-\pi$, and we give here an improved version:

$$
\Delta(\xi)=\left\{\begin{aligned}
-0.825-0.05 z-0.77 z^{2} & \text { for } \xi \in\left[-\pi,-\frac{5}{8} \pi\right] \\
+1.26 z^{3}-0.37 z^{4} & \\
2.11 y+1.96 y^{2}+1.38 y^{3} & \text { for } \xi \in\left[-\frac{5}{8} \pi,-\frac{3}{8} \pi\right] \\
6.027-9.64 x+3.14 x^{2} & \text { for } \xi \in\left[-\frac{3}{8} \pi,-\frac{\pi}{4}\right]
\end{aligned}\right.
$$

where $z=\xi+\pi, y=\xi+\pi / 2$, and $x=\sqrt{-\xi-\pi / 4}$.

The discrete scale invariance not only holds for the three-body bound states, but for the whole three-body spectrum including the scattering continua $[64,65]$. In the three-body scattering continuum for $a<0$, resonances that arise at threshold from the three-body bound states and persist up to energies $\sim 2 \hbar^{2} /\left(m a^{2}\right)$ also exhibit a discrete-scale-invariant pattern with the scaling factor $\lambda_{0}$ [65]. These Efimov resonances have been used to evidence Efimov states in experiments with ultra-cold atoms, as discussed in section 4.7.2. On the other hand, for $a>0$, the disappearance of the three-body bound states below the particle-dimer threshold does not lead to trimer resonances but trimer virtual states, similarly to the disappearance of the two-body bound state below the two-body threshold.

\subsection{Finite-range interactions}

The Efimov effect has been confirmed by many calculations using finite-range interactions [66, 67]. In particular, a series of trimer states is obtained in these calculations. One important aspect of systems with finiterange interactions is that the discrete scale invariance is necessarily broken below some distance comparable with the range of interactions. As a result, the spectrum is bounded from below, as it should be physically: the series of trimer states starts from a ground state.

Finite-range calculations show that in the universal window where the scattering length $a$ is much larger than the range $b$ of interactions, and the wave number $k$ associated with the three-body energy $\hbar^{2} k^{2} / m$ is much smaller than the inverse range $b^{-1}$, the dimer and trimers follow closely the zero-range theory predictions, in particular the energy spectrum follows the discrete scale-invariant Efimov spectrum given by equation (4.39). Outside this window, however, the spectrum deviates from the universal predictions of the zero-range theory. Typically, the ground-state trimer shows marked deviations from the universal spectrum and does not meet the particle-dimer threshold, which 
can be understood from a variational argument [68]. The first excited state may also show some deviations near the particle-dimer threshold, which it approaches closely, following the Efimov scenario, but does not necessarily meet [69]. These features are summarised in figure 4.3 for a three-body system whose two-body attractive potential $V(r)$ is gradually scaled by a strength factor $g$, enabling to change the scattering length and make it resonant for certain values of $g$.

Finite-range corrections Experimental observations often lie on the border of the universal window, where the zero-range theory may not be accurate enough. Some efforts have therefore been devoted to understanding the finite-range corrections to the zero-range theory.

A first line of approach is based on the effective range theory [70]. At the two-body level, deviations from the zero-range theory involve the effective range $r_{e}$, which appears in the low-energy expansion of the $s$ wave phase shift [70]:

$$
\frac{k}{\tan \delta_{0}(k)}=-\frac{1}{a}+\frac{1}{2} r_{e} k^{2}+o\left(k^{2}\right)
$$

The effective range $r_{e}$ is typically, but not always, on the order of the range $b$ of the interactions. It is relatively straightforward to take into account this two-body range correction into the three-body problem. In the three-body Schrödinger formalism, one can apply a generalised Bethe-Peierls boundary condition that replaces the scattering length by the energydependent quantity $-k / \tan \delta_{0}(k)$. This quantity also appears explicitly in the integrated Schrödinger equation of the zero-range three-body problem, known as the Skorniakov- Ter-Martirosian equation [19]. One can therefore use the low-energy expression of the phase shift given by equation (4.41) into these formalisms [71, 72, 73, 74]. This brings out a correction $\propto r_{e} / R^{3}$ to the Efimov attraction of equation (4.33) [71, 72, 73]. One can use more elaborate expressions describing the energy dependence of the phase shift over a wider range of energy $[75,76]$.

It would be tempting to think that such a procedure regularises the Thomas collapse problem of the zero-range theory and sets the three-body parameter through the new length scale given by $r_{e}$ [76]. It is indeed the case for a large and negative effective range, a situation that arises in the case of narrow Feshbach resonances [77] - see section 11.4.3. However, in general the procedure does not regularise the equations, and one still has to impose a regularisation of the equations that introduces a three-body parameter. Such an approach with a fixed three-body parameter has not been quite successful in reproducing experimental data and theoretical calculations with finite-range interactions; an energy dependence of the three-body parameter is needed to reproduce these results [75, 78, 79]. A likely reason is that equation (4.41) only accounts for the range corrections of the phase shift, i.e. the on-the-energy-shell scattering properties, which correspond to asymptotic properties of two-body systems, but not the off-the-energy-shell properties which correspond to their short-range correlations. In this respect, separable potentials [80] are useful tools to account for finite-range effects, since they can reproduce both onand off-the-energy-shell finite-range effetcs, while keeping the simplicity of the zero-range theory [81, 82] - see Appendix for details.

An alternative and more systematic approach to range corrections is based on the effective field theory $[83,84]$. Effective field theory [53] is the effective theory that one can write at low energy respecting the basic symmetries of the systems. In this framework, the ratio $b /|a|$ of the range of interaction over the scattering length can be treated as an expansion parameter. The leading order in this expansion reproduces the zero-range theory [53]. Calculations to the next-to-leading order have been performed in Refs. [85, 86, 84, 87] and show the necessity to introduce a second three-body parameter to renormalise the equation at this order.

A more recent approach $[88,89,90]$ based on numerical calculations with model potentials has provided an empirical way to reproduce range corrections to the zero-range theory. These works show that finiterange deviations from universal formulas such as equation (4.39) can be accounted for to a good accuracy over a wide range of scattering length and energy by simply replacing the scattering length $a$ by a length $a_{B}$, and shifting the three-body parameter by a quantity inversely proportional to $a$. The length $a_{B}$ is defined as the value $\kappa^{-1}$ that is the solution of $\tan \delta_{0}(i \kappa)=-i$, corresponding to the pole of the scattering amplitude $f(k)=\left(k / \tan \delta_{0}(k)-i k\right)^{-1}$, provided that an analytic continuation to imaginary $k$ is possible. For $a>0$, the energy $-\frac{\hbar^{2}}{m a_{B}^{2}}$ therefore coincides with the two-body bound-state energy, while for $a<0$ it corresponds to the energy of a virtual bound state, since there is no physical bound state. This procedure has been used to fit theoretical results obtained with finite-range interactions, as well as experimental data obtained for lithium-7 [90, 36]. According to this procedure, the universal formula (4.39) for the trimer energy is modified as follows (changes are emphasised in red),

$$
\left|E^{(n)}\right|+\frac{\hbar^{2}}{m a_{B}^{2}}=\frac{\hbar^{2}\left(\kappa_{*}+\Gamma_{n} / a\right)^{2}}{m} e^{-2 \pi n /\left|s_{0}\right|} e^{\Delta(\xi) /\left|s_{0}\right|} .
$$

Equivalently, the finite-range energy curve can be mapped to the original Efimov curve by plotting the renormalised energy $E^{\prime(n)}=\lambda_{n}^{2} E^{(n)}$ (or wave number $\left.\kappa^{\prime(n)}=\lambda_{n} \kappa^{(n)}\right)$ as a function of the renormalised inverse scattering length $a^{\prime-1}=\lambda_{n} a_{B}^{-1}$ with the $a$-dependent renormalisation coefficient $\lambda_{n}=$ $\left(1+\Gamma_{n} /\left(\kappa_{*} a\right)\right)^{-1}$. An example of such mapping will be shown in the case of helium- 4 in section 4.7.1. 


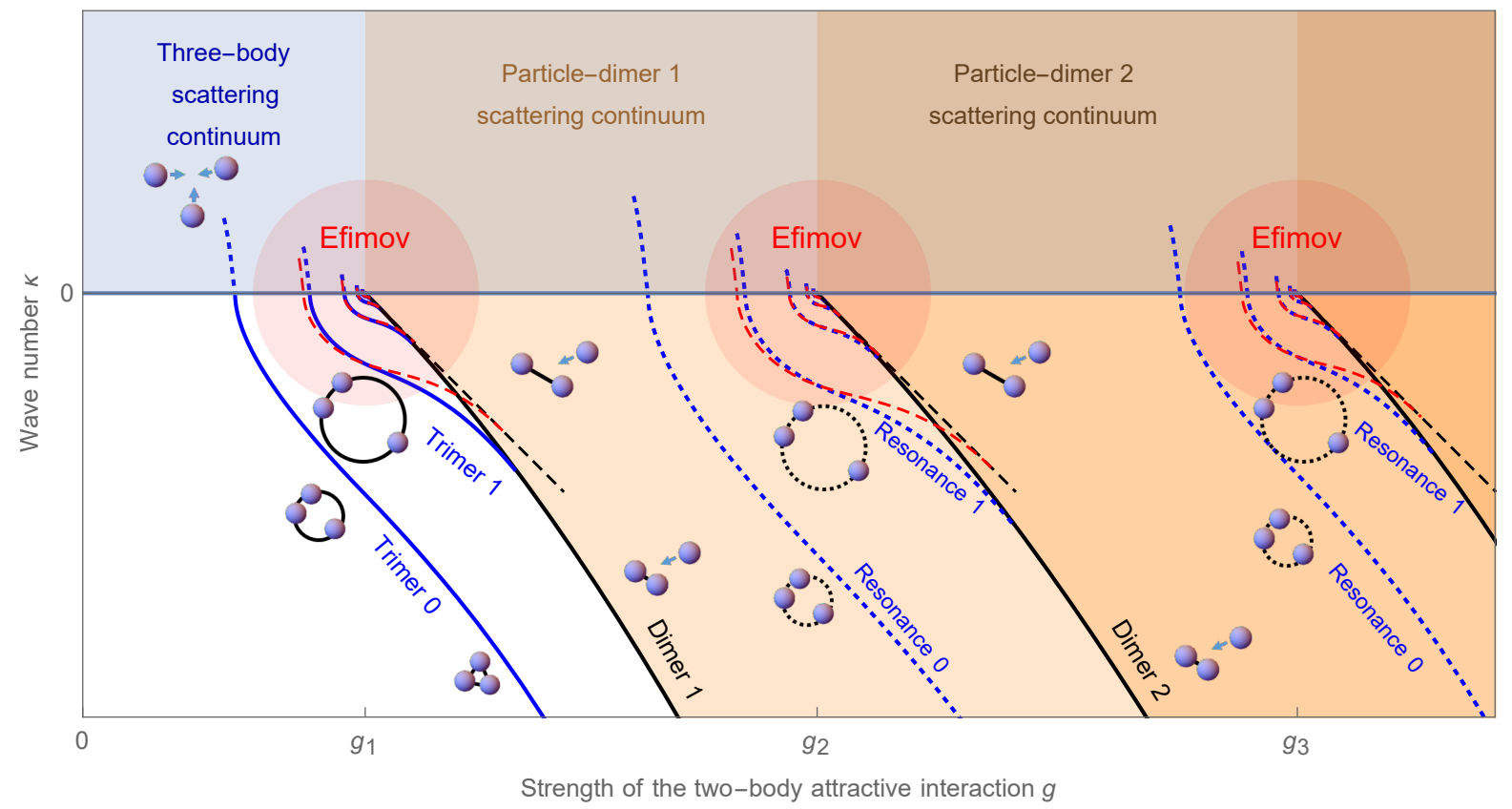

Figure 4.3: Schematic plot of the three-body spectrum of three identical bosons of mass $m$, as the strength $g$ of their two-body attractive interaction $g V(r)$ is increased. For clarity, the wave number $\kappa=E \sqrt{m /(\hbar|E|)}$ is represented instead of the energy $E$, and only states with zero angular momentum are shown. At a certain interaction strength $g_{1}$, an $s$-wave two-body bound state (solid black curve labelled as dimer 1) appears, whose binding energy increases with increasing strength. At larger strengths $g_{2}, g_{3}$, etc., a second, third, etc., $s$-wave two-body bound states (solid black curves labelled as dimer 2, etc.) appear. Just before the appearance of the first two-body bound state, an infinite set of three-body bound states emerge, indicated by solid blue curves. Just before the appearance of the other two-body bound states, a similar set of three-body states appear, indicated by dotted curves. These states are not true bound states, but resonant states embedded in the continua of scattering states between a particle and a deeper two-body bound state. These continua are indicated by the shaded areas above the curves corresponding to the two-body bound states. Around each appearance of a twobody bound state (the "two-body resonances"), the inverse scattering length $1 / a$ is proportional to $g-g_{i}$. As a result, the three-body states follow the Efimov spectrum of figure 4.2, here shown in dashed red curves. These regions of good agreement with the zero-range theory are called "Efimov windows of universality" and indicated by red discs. Away from these regions, the two-body and three-body bound states significantly deviate from the ideal Efimov spectrum.

The replacement $a \rightarrow a_{B}$ is related to the two-body range correction given by equation (4.41). Indeed, according to the definition of $a_{B}$ and to equation (4.41), one has:

$$
\begin{aligned}
\frac{1}{a_{B}} & \approx r_{e}^{-1}\left(1-\sqrt{1-2 r_{e} / a}\right) \\
& \approx \frac{1}{a}\left(1+\frac{1}{2} \frac{r_{e}}{a}+\ldots\right)
\end{aligned}
$$

In contrast, the shift $\Gamma_{n} / a$ is a range correction to the three-body parameter,

$$
\kappa_{*}^{\prime}=\kappa_{*}\left(1+\frac{\Gamma_{n} / \kappa_{*}}{a}+\ldots\right)
$$

that is likely associated with two- and three-body short-range correlations. The form of this shift was recently justified from effective-field theory [91], but the value of $\Gamma_{n}$ has so far been determined only numerically for each value of $n$ to reproduce finite-range calculations. These results suggest that, with the introduction of the parameters $r_{e}$ and $\Gamma_{n}$ characterising finite-range corrections, the universality of Efimov physics may be extended beyond the window of validity of the zero-range theory.

\subsection{Other interactions}

\subsubsection{Coulomb interactions}

Electrically charged particles are subjected to the Coulomb interaction. It is a long-range interaction, whose potential decays as $1 / r$, thus more slowly than $1 / r^{3}$. For such interactions, there is no range beyond which the particles effectively cease to interact. Therefore, there is no Efimov physics associated with Coulomb interactions themselves. However, particles interacting with short-range interactions may also interact with additional Coulomb interactions due to their electric charge. Such is the case of protons or 
nuclei, which interact through the short-range nuclear forces as well as the repulsive Coulomb interaction. If the short-range interactions are resonant, there is an expected interplay between the $1 / R^{2}$ Efimov attraction (4.33) and Coulomb forces.

To our knowledge, this interplay has not been studied explicitly, due to the technical difficulties in solving the three-body problem with Coulomb interactions [92]. Nevertheless, some simple considerations can be made, as discussed by Vitaly Efimov in his original paper [1]. Since the Coulomb potential decays as $1 / r$ and the Efimov attraction decays as $1 / R^{2}$, the latter dominates at short distances and the former dominates at large distances, breaking the scaling invariance. The distance where this transition occurs is given by the Bohr radius

$$
a_{C}=\frac{\hbar^{2}}{m k_{e} q^{2}},
$$

where $m$ is the mass of particles, $q$ is their electric charge, and $k_{e}=1 / 4 \pi \epsilon_{0}$ is Coulomb's constant. On the other hand, the Efimov attraction exists only beyond the range $b$ of the short-range resonant interaction. Therefore, the window of existence for the Efimov attraction is delimited by the range $b$ of the short-range forces and the Bohr radius $a_{C}$. Within this window, Efimov states can be bound by the Efimov attraction, and their number scales as $\ln \left(a_{C} / b\right)$. A necessary condition for the existence of Efimov states in these systems is thus

$$
b<a_{C} .
$$

The single-particle problem in a sum of $1 / r^{2}$ and $1 / r$ potentials was treated quantitavely in reference [93], and corroborates these qualitative considerations.

\subsubsection{Dipolar interactions}

Even if particles are electrically neutral, they may possess an electric or magnetic dipole moment that creates a dipole-dipole interaction between them. In the ultra-cold atom research community, there has been a growing interest in studying particles interacting via dipole-dipole interactions. Prime examples are atoms with a large magnetic dipole moment $\mu$, such as ${ }^{52} \mathrm{Cr}$ $\left(\mu=6 \mu_{B}\right.$, where $\mu_{B} \approx 9.274 \times 10^{-24} \mathrm{~J} \mathrm{~T}^{-1}$ is the Bohr magneton) $[94,95],{ }^{164} \mathrm{Dy}\left(\mu=10 \mu_{B}\right)[96]$, and ${ }^{168} \mathrm{Er}$ $\left(\mu=7 \mu_{B}\right)[97,98]$. Systems with dipolar interactions can also be realised with polar molecules that possesses a permanent electric dipole moment $d$ [99, 100, 101]. For these atoms and molecules, the strength and the polarisation direction of the dipole interaction can be controlled by external magnetic or electric fields, aligning the dipoles in certain directions.

For two particles with dipole moments induced by an external field and aligned along the vertical direction, the dipole-dipole interaction potential at large distance has the form

$$
V(r, \theta)=d^{2} \frac{1-3 \cos ^{2} \theta}{r^{3}}
$$

where $d$ is the dipole moment (expressed in units of $\left.\mathrm{m}^{5 / 2} \cdot \mathrm{kg}^{1 / 2} \cdot \mathrm{s}^{-1}\right), r$ is the distance, and $\theta$ is the polar angle between the two particles. It is thus an anisotropic interaction with a long-range tail. While it is seemingly more complicated than isotropic short-range interactions discussed in sections 4.1 and 4.2, the two-body physics of dipoles turns out to exhibit the same universal behaviour as that of short-range interactions around the threshold regime at which two dipoles are about to form an $s$-wave dominated bound state [102, 103, 104]. Close to such $s$-wave dominated resonances, the coupling between different partial waves induced by the dipole interaction occurs at a distance much smaller than the spatial extent of the bound state since the coupling decays as $1 / r^{6}$. One can therefore essentially consider a single channel scattering in the $s$-wave channel, in which the dipole interaction averages out to zero and one is left with a short-range interaction in this channel. The calculation of this interaction in secondorder perturbation theory (through the coupling of the $s$ wave to the $d$ wave) shows that it decays as $-C_{4} / r^{4}$ with $C_{4}=4 \hbar^{2} \ell_{4}^{2} / m$ and $\ell_{4} \approx 0.365 \ell_{d}[105]^{5}$, where $\ell_{d}$ is the dipole length defined as

$$
\ell_{d}=\frac{m d^{2}}{2 \hbar^{2}}
$$

Because of this similarity of the dipole-dipole interaction with a $1 / r^{4}$ short-range attraction in the $s$ channel, the two- and three-body physics near an $s$-wave dominated two-body resonance shows the same universal behaviour as that of short-range interactions. Namely, near an $s$-wave dominated resonance, the dimer energy scales with the scattering length according to the universal formula (4.5) [104], and the Efimov effect occurs [106]. In reference [106], Yujun Wang and co-workers have considered the three-body problem of identical bosons with the dipole-dipole interaction in the proximity of the $s$-wave dominated resonance. They have found the appearance of Efimov states with the same universal scaling factor 22.7 as that of systems with short-range interactions. One notable feature of the dipolar Efimov states is that their three-body parameter is universally set by the dipole length,

$$
\kappa_{*}^{(0)}=0.173(2) \ell_{d}^{-1},
$$

in the absence of other forces at distances comparable with the dipole length. The insensitivity of the threebody parameter to forces at shorter distances than the dipole length is due to the strong repulsion created by the partial wave couplings induced by the dipole interaction. This repulsion appears at a distance on the order of the dipole length. It prevents the three dipoles from getting closer than the dipole length and renders the three-body parameter universal.

\footnotetext{
${ }^{5}$ The value of $\ell_{4} \approx 0.365 \ell_{d}$ is derived from equation (34) in reference [105], as we could not reproduce the value $2 \ell_{4}=1.09 \ell_{d}$ given after equation (34) of the same reference.
} 
As we shall see in sections 11.2 and 11.3, a threebody repulsion also appears in systems with isotropic short-range interactions, in particular power-law decaying interactions, and makes in some limit the threebody parameter universally determined by the effective range. It would be therefore tempting to think that the three-body universality of the dipole-dipole interaction is related to the three-body universality of its effective $1 / r^{4}$ interaction in the $s$ wave channel. However, the universal three-body parameter for such an $1 / r^{4}$ interaction has been estimated to $\kappa_{*}^{(0)} \approx 0.174 r_{4}^{-1} \approx$ $0.48 \ell_{d}^{-1}$ [82], which is almost a factor of three different from the value in equation (4.49). This indicates that the dipole-dipole interaction belongs to a different class of three-body universality that involves the explicit partial wave coupling at short distance. We will discuss the universality classes of the three-body parameter for isotropic short-range interactions in more detail in section 11.3.

The dipolar three-body physics leads to an even more interesting behaviour when the particles are identical fermions. While identical fermions do not exhibit Efimov physics (see section 5), Yujun Wang and coworkers have found in reference [107] that there exists a new type of three-body bound state, which is universally described by the dipole interaction. The size of this bound state is of the order of the dipole length, and it has a shape of an obtuse isosceles triangle, whose longer side is parallel to the polarisation axis of the dipoles. This particular shape originates from a competition between the dipole interaction and the Pauli exclusion principle, maximising the attraction between the dipoles by aligning them in parallel while preserving the antisymmetrisation condition by having nodes between them.

\subsubsection{Inverse-square interactions and generalised Efi- mov effect}

The scale invariance of Efimov physics comes from the $1 / r^{2}$ dependence of the Efimov attraction, which scales like the kinetic energy. This long-range three-body attraction is remarkable because it originates from shortrange two-body interactions. If the two-body interactions are not short-ranged but have an attractive $1 / r^{2}$ dependence themselves, then the three-body system is also expected to feature an effective scale-invariant three-body attraction. In such systems, however, the long-range nature of the forces is set by construction, and does not emerge from short-range forces, as in the Efimov effect. In this sense, they may not be considered to be related to the Efimov effect. On the other hand, an interesting question about these systems is whether they exhibit a discrete scale invariance that requires an extra length scale, as in the Efimov effect. Concretely, the question is whether the $-\alpha / r^{2}$ two-body potentials are strong enough to support two-body and three-body bound states. For two-body systems, $\alpha$ must be larger than $\alpha_{2}=\hbar^{2} /(8 \mu)$, where $\mu$ is the particles' reduced mass. In this case, there is an infinity of two-body bound states whose energy spectrum forms a geometric series as in the Efimov effect. This breaks the scaling invariance into a discrete scaling invariance and requires the knowledge of a microscopic length scale ${ }^{6}$. Likewise, the three-body spectrum features an infinite number of bound states or resonances below each twobody bound state. The problem becomes particularly interesting for $\alpha<\alpha_{2}$, for which the attraction is not strong enough to bind two particles, but may be strong enough to bind three particles. In this case, the energy spectrum of these systems resembles that of Efimov systems, in the sense that an infinity of discretescale-invariant three-body states may exist despite the absence of two-body bound states. For this reason, Sergej Moroz, José D'Incao and Dmitry Petrov [108] have advocated a generalised definition of the Efimov effect, as "the emergence of discrete scaling symmetry in a three-body problem if the particles attract each other via a two-body scale-invariant potential", regardless of the short- or long-range nature of this potential. Situation in 3D The situation of three identical particles interacting via inverse-square potentials was studied by Nicolais L. Guevara, Yujun Wang, and Brett D. Esry [109]. For identical bosons, they found that the generalised Efimov effect can occur slightly below the critical strength required for binding two particles, namely for $\alpha_{3}<\lesssim \alpha<\alpha_{2}$, with $\alpha_{3}=0.97 \alpha_{2}$. In this range, an infinite family of three-body bound states exists. Their energies form an almost geometric series, as the effective three-body potential turns out to be almost but not exactly an inverse-square potential. One must note that these states are extremely weakly bound, and their scaling ratio is typically on the order of $10^{10}$. For $\alpha>\alpha_{2}$, an infinite number of twobody bound states arise, and the three-body bound states remain below the lowest two-body bound state, while new families of three-body resonances exist below each two-body bound state. Interestingly, similar results were obtained for three identical fermions in the $1^{+}$symmetry, with $\alpha_{2}=9 \hbar^{2} /(8 \mu)$ and $\alpha_{3}=0.82 \alpha_{2}$, whereas the standard Efimov effect with short-range interactions does not apply to identical fermions (see section 5).

Situation in 1D In one dimension, the problem of three identical particles interacting via attractive inverse square potentials was solved analytically by $\mathrm{F}$. Calogero [110] who found that the system remains scale-invariant for $\alpha<\alpha_{2}$, i.e. there is no generalised Efimov effect. However, the situation changes if one considers a particle interacting via an inverse square two-body potential with two identical particles.

\footnotetext{
${ }^{6} \mathrm{As}$ in the Efimov effect, this length scale originates from the short-range details of the interactions, which inevitably depart from the inverse square form assumed at larger distance, since the purely inverse-square potential has no ground state in this case and is therefore unphysical.
} 
In this case, Sergej Moroz, José D'Incao and Dmitry Petrov [108] found that for any value of $\alpha_{2} / 2<\alpha<\alpha_{2}$, the generalised Efimov effect occurs for a sufficiently large mass ratio. It can also occur for smaller values of $\alpha$ by a fine tuning of the short-range details of the two-body interactions. As a possible physical realisation, the authors have proposed a system of two polar molecules interacting with an electron, all confined along a line.

Connection with the standard Efimov effect In addition to the generalised definition of the Efimov effect, systems with $1 / r^{2}$ interactions may also be interesting from the point of view of the standard Efimov effect. Indeed, $N$-body systems with $1 / r^{2}$ pairwise interactions could constitute in some limit an approximation of systems of $N$ particles undergoing a $1 / r^{2}$ Efimov attraction that is induced by their resonant (short-range) interaction with a lighter particle (see section 6.2). This limit requires that the $N+1$ system may be treated in the Born-Oppenheimer approximation (the light particle being much lighter than the $N$ particles) and that the resulting Born-Oppenheimer potential between the $N$ particles may be approximated by a sum of pairwise $1 / r^{2}$ attractive potentials. This idea was introduced in reference [109], where the authors argued that the four-body Efimov states found in a 3 heavy fermions +1 particle system (see reference [111] and section 13.2) may be described by the $1 / r^{2}$ interaction model of three identical fermions. As mentioned above, this model indeed predicts the existence of a geometric series of three-body bound states in the $1^{+}$symmetry above a critical strength $\alpha_{3}$, which would correspond to a mass ratio of 11.58 in the $3+1$ system. This is qualitatively similar to the appearance of four-body bound states in the $3+1$ system above the critical mass ratio 13.384 [111]. The authors thus interpret the fourbody Efimov effect found in reference [111] as a threebody generalised Efimov effect for inverse-square interactions, that originate themselves from the three-body Efimov effect. It is however unclear to what extent this appealing picture is valid, since the Born-Oppenheimer potential between the three heavy particles is, strictly speaking, different from the sum of pair-wise $1 / r^{2}$ interaction. In particular, applying the same approximation to the 3 heavy bosons +1 particle system, one expects an infinite number of four-body bound states tied to each Efimov trimer state, whereas numerical studies have so far found at most two four-body bound states (see section 12.1 and figure 12.2).

\subsection{Relativistic case}

The Efimov effect, seen as the infinite accumulation of three-body bound states with smaller and smaller binding energies in the three-body spectrum, is by definition a low-energy phenomenon. It is thus not directly affected by relativity. Nevertheless, if the range of inter- actions between particles is smaller than their Compton wave length, relativistic corrections may affect the most deeply bound Efimov states and the three-body parameter. In particular, when the range of interactions is so small that they can be approximated by contact interactions, there is still a length scale in the relativistic theory, the Compton wave length, that may prevent the Thomas collapse and set the three-body parameter, instead of the interaction itself.

The first authors to look at the Efimov effect in a relativistic framework were James V. Lindesay and $\mathrm{H}$. Pierre Noyer in the 1980s [112, 113]. They considered three bosons of rest mass $M$, interacting with attractive contact interactions such that the total energy $M_{2}$ of two particles may be less than $2 M$. They obtained the following integral equation ${ }^{7}$ for the three-body en$\operatorname{ergy} M_{3}$ :

$$
\begin{aligned}
W(k)= & -\frac{1}{\pi} \int_{0}^{\frac{M_{3}^{2}-M^{2}}{2 M_{3}}} \frac{d k^{\prime}}{\varepsilon^{\prime}} \frac{k^{\prime}}{k} \frac{\sqrt{s^{\prime}}}{\frac{1}{a}-\sqrt{M^{2}-\frac{s^{\prime}}{4}}} \\
& \times \ln \left(\frac{\sqrt{M^{2}+\left(k+k^{\prime}\right)^{2}}+\varepsilon+\varepsilon^{\prime}-M_{3}}{\sqrt{M^{2}+\left(k-k^{\prime}\right)^{2}}+\varepsilon+\varepsilon^{\prime}-M_{3}}\right) W\left(k^{\prime}\right)
\end{aligned}
$$

where $\varepsilon=\sqrt{k^{2}+M^{2}}, \varepsilon^{\prime}=\sqrt{k^{\prime 2}+M^{2}}, s^{\prime}=M_{3}^{2}+$ $M^{2}-2 M_{3} \varepsilon^{\prime}$, and the scattering length $a$ is given by $1 / a= \pm \sqrt{\left|M^{2}-M_{2}^{2} / 4\right|}$ where \pm is the sign of $2 M-$ $M_{2}$. For $k / M \ll 1$ and $\left|3 M-M_{3}\right| \ll M$, one retrieves the non-relativistic integral equation. However, unlike the nonrelativistic equation, the integral above has a finite upper limit of integration, which comes from the relativistic kinematics. This prevents the Thomas collapse and the three-body energy is set by the rest mass $M$ (or equivalently the Compton wavelength $h / M c$ ). From this equation, the authors concluded that the Efimov scenario is qualitatively unchanged. The groundstate trimer appears for $M_{2} \approx 2.006 M$ and its energy at unitarity $\left(M_{2}=2 M\right)$ is $\approx 2.988 M$, i.e. it is bound by an energy $\approx 0.0122 M$ with respect to the threebody threshold $3 M$. It disappears below the particledimer threshold at $M_{2} \approx 1.03 M$. The first excited trimer is bound by $2.41 \times 10^{-5} \mathrm{M}$ at unitarity, which is about a factor 507 from the ground state, relatively close to the non-relativistic scaling ratio $e^{2 \pi /\left|s_{0}\right|} \approx 515$ for excited states.

The same problem was independently addressed a few years later by Tobias Frederico [114] in the lightfront dynamics formalism [116]. The author derived

\footnotetext{
${ }^{7}$ Note that in both references $[112,113]$, the equation has the wrong factor $4 \pi$ instead of $1 / \pi$. In the first paper [112], the numerical calculations were performed with this wrong factor and are therefore incorrect for identical bosons.
} 


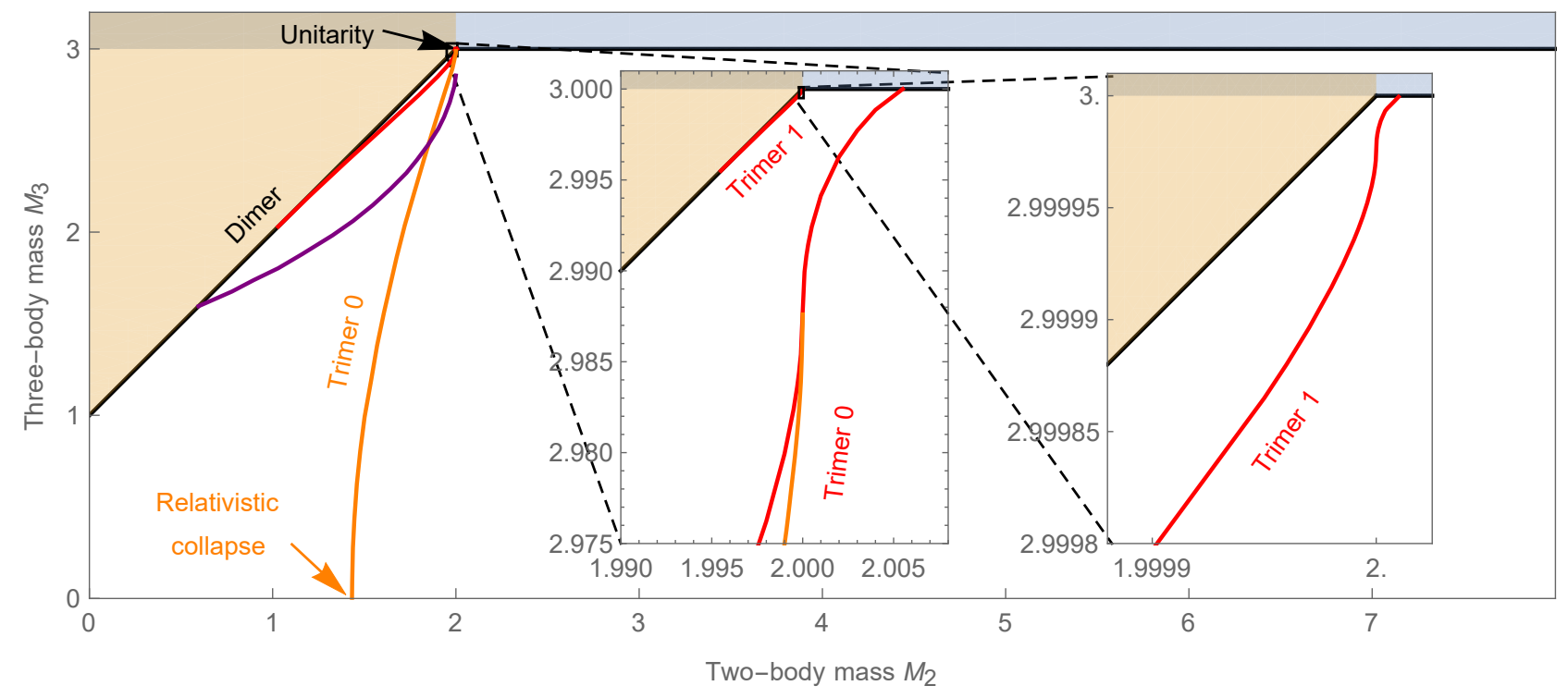

Figure 4.4: Relativistic Efimov spectrum: mass $M_{3}$ of the three-body state as a function of the mass $M_{2}$ of the two-body state in units of the particles' mass $M$. The two-body state is bound for $M_{2}<2 M$. Note that the direction of the horizontal axis is inverted with respect to that of Figs. 4.2 and 4.3. The horizontal line shows the three-body threshold at $M_{3}=M+M+M$ and the diagonal line shows the dimer-particle threshold $M_{3}=M_{2}+M$. The three-body states are bound below these two thresholds. At their intersection is the unitary point $\left(M_{2}=2 M, M_{3}=3 M\right)$ below which the Efimov states accumulate, as shown in the insets. The curve in red shows the results of reference [113], based on equation (4.50). The curve in purple shows the results of reference [114], based on equation (4.51) with $x_{\min }=M^{2} / M_{3}^{2}$ and $q_{\max }=\sqrt{\left(1-x^{\prime}\right)\left(M_{3}^{2} x^{\prime}-M^{2}\right)}$. The curve in orange shows the result of reference [115], based on equation (4.51) with $x_{\min }=0$ and $q_{\max }=\infty$. In the insets, only the excited trimer obtained from equation (4.50) is shown.

the following integral equation,

$$
\begin{aligned}
\Gamma(q, x)= & F\left(M_{12}\right) \frac{1}{(2 \pi)^{3}} \int_{x_{\min }}^{1-x} \frac{d x^{\prime}}{x^{\prime}\left(1-x-x^{\prime}\right)} \\
& \times \int_{0}^{q_{\max }} \frac{d^{2} q^{\prime}}{\mathcal{M}^{2}-M_{3}^{2}} \Gamma\left(q^{\prime}, x^{\prime}\right)
\end{aligned}
$$

where $F\left(M_{12}\right)=8 \pi^{2}\left(\frac{\arctan y_{M_{12}}}{y_{M_{12}}}-\frac{\arctan y_{M_{2}}}{y_{M_{2}}}\right)^{-1}$ with $y_{m}=\frac{m}{\sqrt{4 M^{2}-m^{2}}}$ and $M_{12}^{2}=(1-x) M_{3}^{2}-\frac{q^{2}+(1-x) M^{2}}{x}$, and $\mathcal{M}^{2}=\frac{q^{\prime 2}+M^{2}}{x^{\prime}}+\frac{q^{2}+M^{2}}{x}+\frac{\left(\vec{q}^{\prime}+\vec{q}\right)^{2}+M^{2}}{1-x-x^{\prime}}$. Here, the integral boundaries are set to $x_{\min }=M^{2} / M_{3}^{2}$ and $q_{\max }=\sqrt{\left(1-x^{\prime}\right)\left(M_{3}^{2} x^{\prime}-M^{2}\right)}$. Like equation (4.50), this equation also reduces to the non-relativistic integral equation in the non-relativistic limit. Solving this equation, the author reached conclusions similar to those of Refs. [112, 113], with relatively different numerical results ${ }^{8}$. In particular, the ground-state trimer is bound by about $\approx 0.2 M$ at unitarity and disappears below the particle-dimer threshold at $M_{2} \approx 0.6 M$.

In a more recent work using a similar formalism [115], Jaume Carbonell and V. A. Karmanov argued that for zero-range interactions the boundaries of the integrals assumed in the previous work [114]

\footnotetext{
${ }^{8}$ Note that the numerical results of reference [114] are not converged, as mentioned in reference [115]. We give here the numerical values from reference [115]
}

should be changed to $x_{\min }=0$ and $q_{\max }=\infty$. This results in a drastically smaller binding energy at unitariy $\approx 0.012 M$ for the ground state, which is remarkably close to the results of Lindesay and Pierre Noyer in reference [113]. In addition, the ground-state trimer does not disappear below the particle-dimer threshold. Instead, its energy vanishes at $M_{2} \approx 1.43 M$, which the authors called the "relativistic Thomas collapse". For a smaller mass $M_{2}$ than this critical value (i.e. a stronger two-body attraction), the three-body energy $M_{3}$ is formally imaginary, making it unphysical.

The results of these works are summarised in figure 4.4 .

\subsection{What is an Efimov state?}

Before we address the observations of Efimov states, we have to ask ourselves what is an Efimov state, and what constitutes an experimental evidence of such a state. The answer to these questions varies somewhat from one person to the other. The major issue is whether the ground-state trimer, which is the most likely to exist and most easily observable, should be included or not in the series of Efimov states. 


\subsubsection{Energy}

Let us review some proposed definitions of Efimov states based on their energy spectrum.

1. In the strictest sense, Efimov states are evidence of the Efimov effect, i.e. an accumulation point in the three-body spectrum at zero energy as shown in figure 4.2. In this sense, one cannot evidence a single Efimov state, one needs to exhibit several (at least two) of these states and show that they follow the predicted discrete scale invariant pattern. In this definition, the observation of the ground-state trimer is not an evidence of an Efimov state.

2. In another definition, one may allow a single state to qualify as an Efimov state, if it can be shown that its variation with scattering length follows qualitatively the universal curve obtained in the zero-range theory and shown in figure 4.2, sometimes referred to as the "Efimov scenario". Namely, the trimer has to appear from the threebody threshold at some negative scattering length, and disappear in (or approach closely) the particle+dimer threshold at some positive scattering length. The ground-state trimer often remains far below the particle+dimer threshold [68, 117, 118], and thus does not qualify as an Efimov state in this definition [119].

3. In their review article [4], Eric Braaten and HansWerner Hammer advocated a broader definition: "a trimer is defined to be an Efimov state if a deformation that tunes the scattering length to $\pm \infty$ moves its binding energy along the universal curve". In this definition, the trimer does not have to meet the particle+dimer threshold on the positive scattering length side. The ground state trimer is therefore usually an Efimov state according to this definition.

4. The results of Refs. [88, 89] suggest a somewhat related definition: a trimer is defined to be an Efimov state if the trimer energy as a function of $a_{B}$ can be fitted by the modified universal formula (4.42). The authors of Refs. [88, 89] have presented numerical evidence that close to unitarity the ground state is expected to be an Efimov state in this definition.

The first two definitions are qualitative: a given state is either an Efimov state or not. The last two definitions are less restrictive and can be made quantitative: by comparing the energy with the universal or modified universal formulas, one may quantify how much of an Efimov state a given trimer is. The disadvantage of definitions 2,3 , and 4 is that they require the variation of the two-body scattering length, which is not always possible experimentally if the interactions cannot be controlled but are just set by nature.
From a physical point of view, it would be natural to say that a trimer is an Efimov state if the Efimov attraction is present and necessary to ensure its binding, although this point is difficult to characterise experimentally, and even theoretically for real systems.

We should mention that the notion of Efimov trimer is not restricted to bound states and can be extended to resonant states. When the two-body interaction potential supports several two-body bound states and one of them has zero angular momentum and is very close to threshold, it leads to a situation similar to what the Efimov theory predicts for just one two-body bound state, except that the Efimov trimers are resonant states that can dissociate into a particle and a deeper two-body bound state. Such resonant states are shown as dotted curves in figure 4.3. As long as this dissociation is weak, the trimer resnonances are narrow and follow the Efimov scenario. They can therefore be qualified as Efimov states. In fact, it is the situation encountered in ultra-cold atomic gases - see section 4.7.2. For very strong losses by dissociation, the trimer resonances are broad and a theoretical study indicates that the Efimov spectrum is rotated in the complex energy plane [120], where the imaginary part of the energy correspond to the resonance width.

\subsubsection{Structure}

Although the peculiar energy spectrum of Efimov states is often presented as their defining characteristic, their spatial structure is also worth considering, as it makes them very different from other three-body bound states such as water or ozone molecules. Ozone molecules, for instance, have a relatively well defined geometry, with an O-O length of about $0.127 \mathrm{~nm}$ and an O-O-O angle of about $117^{\circ}$. Although the electrons are delocalised around the oxygen nuclei, the relative positions of the nuclei are quite localised, as a result of the strong binding interaction provided by the bonding electrons. Efimov states, on the other hand, are very diffuse objects without a well defined geometry.

In the intuitive picture of the Efimov attraction, the particles keep moving back and forth between one another, thereby inducing the Efimov attraction that keeps them together. Thus, for an Efimov state made of atoms, one may say that the atoms themselves play the role of bonding electrons by performing an exchange motion. The electrons play a role only when the atoms come in contact, within the radius of their electronic cloud, which is on the order of a few tenths of nanometre for light atoms. The motion of the atoms, on the other hand, occurs at distances larger than the size of their electronic cloud, around three times for the ground state and much more for excited states. As result, Efimov states of atoms are much larger and diffuse than usual molecules. This is shown in figure 4.5 for the case of helium- 4 trimers, whose excited state is typically fifty times or more larger than ozone molecules.

In addition to the broad distribution of sizes, there 


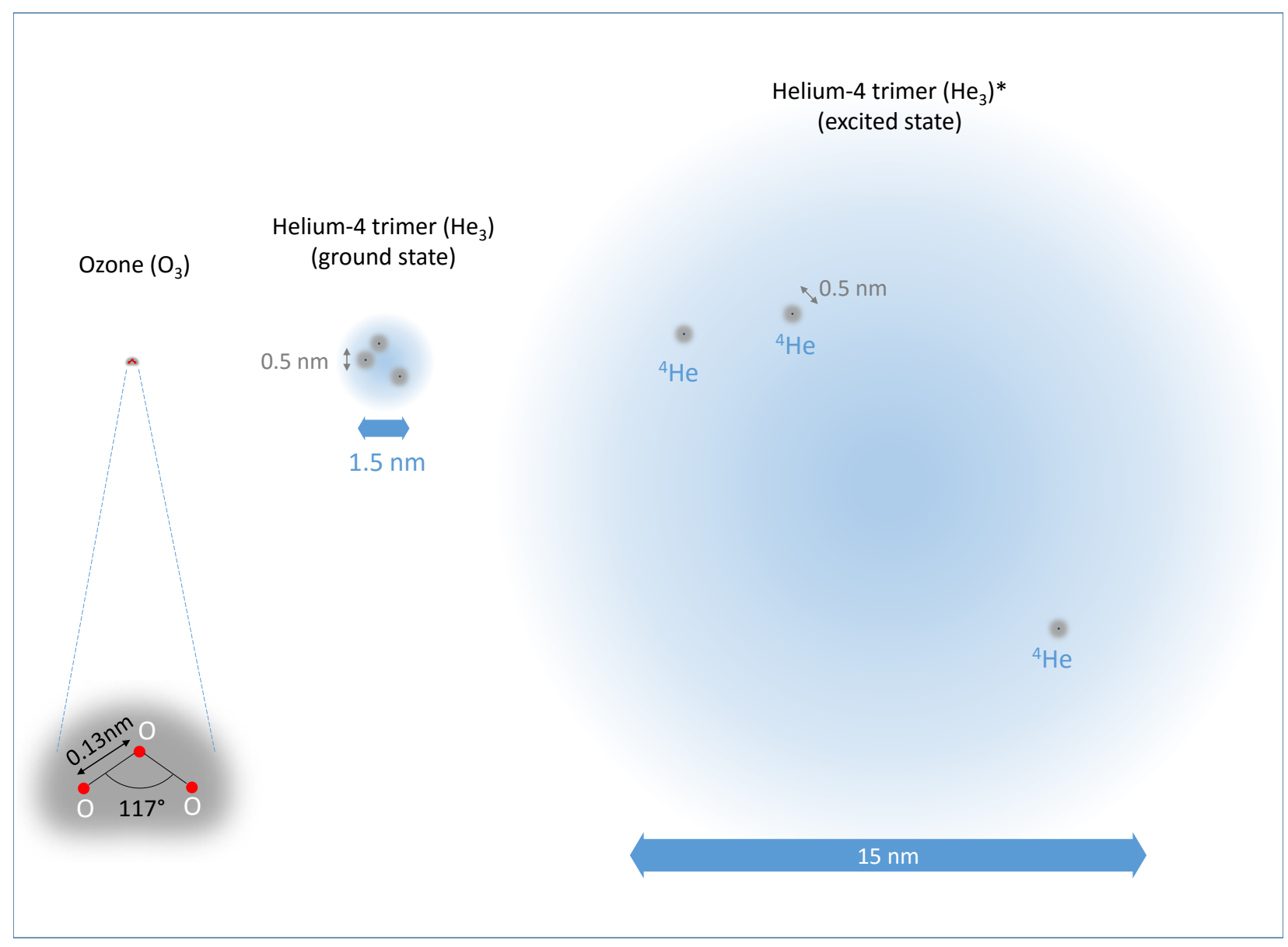

Figure 4.5: Ozone molecule $\left(\mathrm{O}_{3}\right)$ compared to Efimov states of helium-4 atoms $\left(\mathrm{He}_{3}\right)$ on the same scale. Each molecule is represented schematically as a typical snapshot of its geometry. The ozone molecule is enlarged to show its structure (readers of the electronic version are invited to zoom in). The nuclei are represented by small dots (red for oxygen, blue for helium), and the electronic cloud is shown as a grey halo. For the helium trimers, the atomic cloud formed by the motion of the helium atoms is shown as a light blue halo. The structure of the excited helium trimer is closest to that of an ideal Efimov state, which is typically described by an elongated triangle configuration. The structural properties of the ground helium trimer do not conform fully to those of Efimov states, but are nonetheless very different from more compact molecules like $\mathrm{O}_{3}$.

is also a broad distribution of geometries for three particles forming an Efimov state. The most probable geometries in this distribution correspond to elongated triangles, where two particles are relatively close and the third one is farther away. This can be checked at unitarity from the hyper-angular wave function in equation (4.28), which peaks at $\alpha=0$, corresponding to two particles in contact with the third particle away.

These distinctive structural properties can be used to experimentally characterise a trimer as an Efimov state, when structural properties can be measured. This was recently demonstrated in the Coulomb explosion imaging of helium-4 trimers [121, 12] - see section 4.7.1. This experiment revealed that the distribution of geometries for the excited trimer state of helium-4 conforms to the distribution of an Efimov state favouring elongated triangles, whereas the distribution for the ground state is broader and does not seem to favour any particular geometry. This is due to the fact that finite-range effects are more important for the ground state and tend to push the system to a more equilateral configuration (see a discussion of this effect in section 11.2.1). For this reason, groundstate Efimov trimers are in general not expected to exhibit the distribution of geometries of an ideal Efimov state. Nevertheless, the absence of a well-defined geometry make even the ground-state Efimov trimers very distinct from conventional molecules, as figure 4.5 illustrates.

\subsection{Observations in nuclear physics}

Bosonic particles in nuclear physics are compounds of fermions. In the case of nucleons, since neutrons do not bind, bosonic clusters have to involve protons. This introduces Coulomb interactions which do not qualify as short-range interactions. Nevertheless, as discussed in section 4.3.1, Efimov physics can survive in this con- 


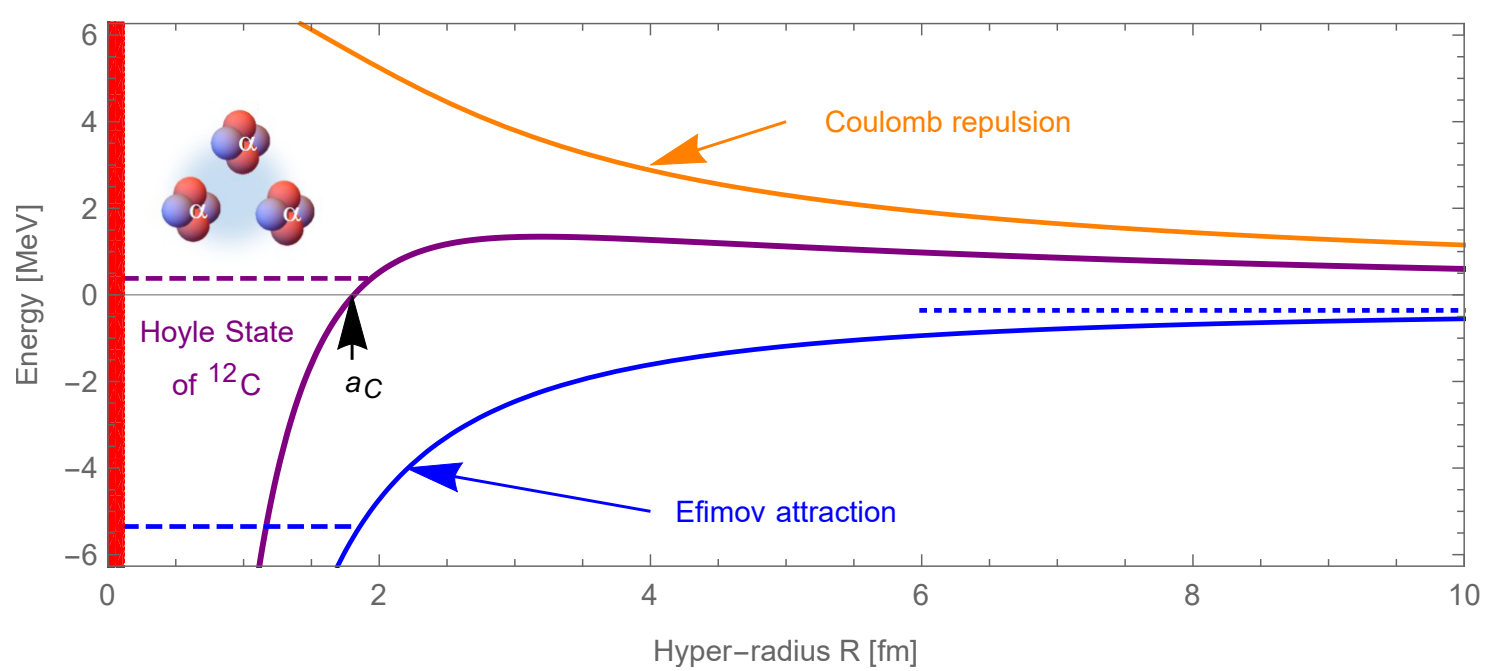

Figure 4.6: Schematic picture of the conjecture that the Hoyle state of carbon-12 is bound by the Efimov attraction. In this picture, the Hoyle state appears as a resonant state supported by the sum of two potentials as a function of the hyper-radius between the three alpha particles. The first potential is a Coulomb repulsive potential, here taken to be $-\alpha(2 e)^{2} k_{e} \operatorname{erf}(R / \beta) / R$ where $\alpha=2$ and $\beta=1.66 \mathrm{fm}$, and represented in orange. The second potential is an Efimov attractive potential shown in blue, asymptoting at small hyper-radius to equation (4.33) and at large hyper-radius to the dimer energy $-\hbar^{2} / m a^{2}$ shown by the dotted blue line), where the alpha-alpha scattering length $a=5.4 \mathrm{fm}$. The sum of the two potential is shown in purple. A three-body repulsive hard wall shown in red is set at $R_{0}=0.122 \mathrm{fm}$ to fix the three-body parameter and reproduce the energy of the Hoyle state at $0.38 \mathrm{MeV}$, shown by the dashed purple line. The energy of that state without Coulomb repulsion is shown by the dashed blue line. Note that the value of $R_{0}$ is much smaller than the range of the alpha-alpha interaction, and thus quite unlikely.

text: if the short-range nuclear interactions are resonant, they create a three-body Efimov attraction that competes with the Coulomb repulsion to form bound states. Although these states do not necessary follow the definitions of Efimov states put forward in section 4.5.1, they could still be regarded as Efimov states in the sense of being bound by the Efimov attraction. A necessary condition for the Efimov attraction to survive is given by equation (4.46). This condition can only be satisfied by light nuclei.

\subsubsection{The Hoyle state of carbon-12}

The Hoyle state is an excited resonant state of carbon12 predicted by Fred Hoyle in 1954. It plays a crucial role in the stellar nucleosynthesis of carbon. In his original papers [1, 2], Vitaly Efimov suggested that the Hoyle state may be viewed as a trimer of alpha particles (i.e. helium nuclei, which are bosons) bound by the Efimov attraction. The works of Renato Higa and Hans-Werner Hammer [122, 93] based on EffectiveField Theory looks into the effect of the Coulomb interactions on alpha systems close to unitarity. They conjectured that the Hoyle state is indeed a remnant of the Efimov spectrum broken by the Coulomb interaction, surviving as a resonance above the three-alpha scattering threshold. The corresponding picture of the Hoyle state would be a resonant state resulting from the balance between the Efimov attraction and the Coulomb repulsion. This picture is shown in figure 4.6.

Although this picture of the Hoyle state is quite appealing, there are two points that make it questionable. First, excluding the Coulomb repulsion, the nuclear force between two alpha particles does not seem to be resonant, as the scattering length of the model potentials [124] for the alpha-alpha interaction is about $5 \mathrm{fm}$, which is similar to the range $b$ and effective range $r_{e} \approx 3.4 \mathrm{fm}$ of these potentials. The resonance condition (4.4) may therefore not be satisfied. This would suggest that the attraction between alpha particles is directly due to the nuclear force rather than the Efimov attraction. Second, even if the alpha-alpha interaction is resonant, the Efimov attraction seems too weak to overcome the Coulomb repulsion and support a resonant state at distances larger than the range $b$. Indeed, the value of the Bohr radius given by equation (4.45) for alpha particles is $a_{C} \approx 1.8 \mathrm{fm}$. The condition $b<a_{C}$ of equation (4.46) therefore does not appear to be satisfied.

These conclusions rely on rough estimates, and only a full treatment of the three-body problem with shortrange and Coulomb interactions can give a definite answer. The three-body model calculation of the Hoyle state by Hiroya Suno, Yasuyuki Suzuki, and Pierre Descouvemont [125] gives a preliminary answer. In their work, they show the contributions from the Coulomb, nuclear and centrifugal (kinetic) energies as a function of the hyper-radius. Although an attractive well (pre- 


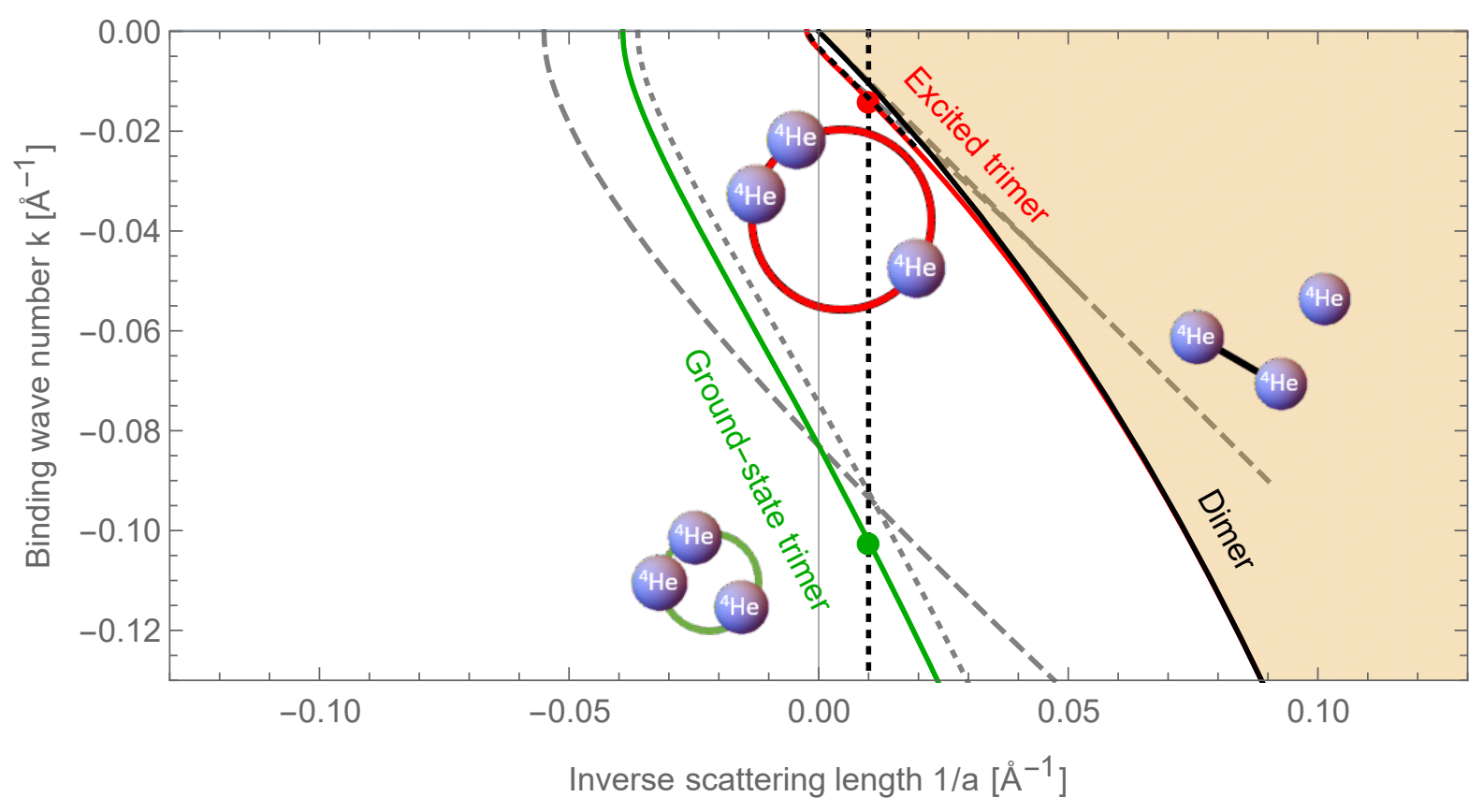

Figure 4.7: Efimov plot for three helium-4 atoms, showing the wave number $k=E\left(\frac{m}{|E| \hbar^{2}}\right)^{1 / 2}$ as a function of the inverse scattering length $1 / a$ artificially varied by scaling the interaction potential for two helium- 4 atoms (the potential used is the so-called LM2M2 potential of reference [123]). The dimer state is shown in solid black, while two trimer states are shown in green (ground) and red (excited). The vertical dotted line shows the value of the scattering length for the unscaled potential, i.e. the physical value for the true helium- 4 system, and the corresponding trimer energies are shown by the dots. They roughly follow the structure of the zero-range Efimov spectrum, shown by the dashed curves. The dotted curves show the trimer energies obtained for a separable interaction reproducing exactly the two-body wave function at zero energy of the scaled helium-4 potential (see Appendix).

sumably due to the Efimov attraction) can be seen in the centrifugal energy, it appears that it is not enough to overcome the Coulomb repulsion, and it is the nuclear force that is responsible for the stability of the Hoyle state in this model. It is therefore likely that the Hoyle state may not be considered as an Efimov state.

\subsection{Observations with atoms}

Most atomic species have bosonic isotopes, and interactions between neutral atoms is of the short-range type, decaying with a $1 / r^{6}$ van der Waals tail. They therefore appear as ideal systems for the observation of the Efimov physics of identical bosons described previously. However, the remaining condition that the interaction be resonant is more difficult to satisfy.

In most cases, the scattering length is on the order of the range of the atomic interaction. Only in accidental cases does the scattering length happen to be much larger than the range of the interaction. Such is the case of helium-4 atoms discussed below. For a long time, helium- 4 appeared as the only promising atomic species for observing Efimov states.

The situation changed drastically with the implementation of magnetic Feshbach resonances in ultracold atoms experiments [126]. Thanks to these res- onances, it has been possible to change at will the scattering length between various species of atoms by simply varying the strength of an applied magnetic field. This has enabled a detailed exploration of Efimov physics in these atomic systems.

\subsubsection{Helium-4}

Theoretical predictions Because of its simplicity, the interaction potential of atomic helium has been widely studied. The range of this potential can be characterised by its van der Waals length, which is about $0.27 \mathrm{~nm}$ and its scattering length turns out to be about 20 times larger than this range, around $10 \mathrm{~nm}$. This fact makes helium-4 systems close to the resonance condition (4.4) for Efimov physics. It was first noted by T. K. Lim and co-workers [25] who suggested that helium-4 atoms may exhibit the first example of Efimov trimer in nature, based on approximate threebody calculations. This triggered many subsequent three-body calculations using refined methods and helium potentials $[127,117,128]$ (see also references in [128]). These calculations predicted the existence of two helium-4 trimers. The one with lowest binding energy (the excited trimer) was identified as an Efimov trimer, in accordance with the definitions of sec- 


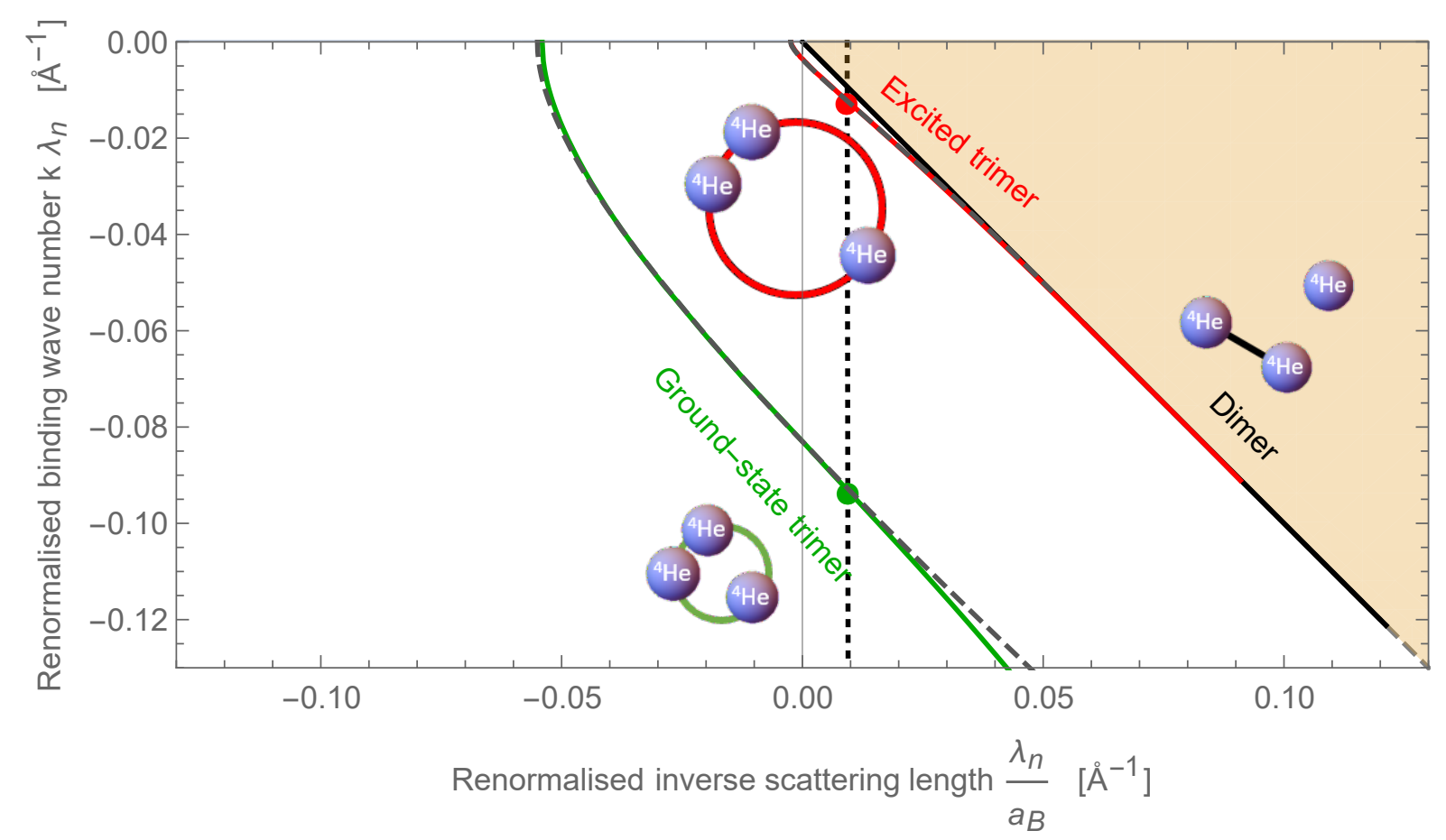

Figure 4.8: Same figure as figure 4.7, where the scattering $a$ is length is replaced by the length $a_{B}$, and both axes are renormalised by the $a$-dependent factor $\lambda_{n}=\left(1+\frac{\Gamma_{n}}{\kappa_{*} a}\right)^{-1}$. The values of $\Gamma_{n}$ are adjusted to $\Gamma_{0}=0.79$ and $\Gamma_{1}=0.99$ to best reproduce the Efimov spectrum curves (shown by the dashed curves).

tion 4.5. The ground-state one has not been regarded as an Efimov trimer, because its energy does not fit well in the discrete scale-invariant pattern of the Efimov universal theory. According to definitions 3 and 4 of section 4.5, however, the ground-state trimer is also an Efimov state.

To appreciate how the two trimer states of helium-4 fit in the Efimov picture, one can change the scattering length between two helium atoms by scaling their interaction potential by some variable factor, and plot the trimer energies as a function of the varied scattering length. This is represented in Fig. 4.7. When the scattering length is tuned to infinity, the Efimov effect occurs and an infinite number of trimer states exist. At the physical value of the scattering length, only two trimer states remain, below the atom-dimer threshold. As the scattering length is varied, these two trimer states roughly follow the Efimov scenario, although none of them dissociate into the atom-dimer threshold for the helium potential used in figure 4.7. As expected, the ground-state trimer shows the most marked deviations. The excited trimer energy comes very close but always remains below the dimer energy, which cannot be seen in figure 4.7 .

The significant deviations of the dimer and trimer energies with respect to the ideal Efimov spectrum can be accounted for by finite-range corrections. One can for instance replace the zero-range interaction by a separable interaction parameterised to reproduce exactly the scattering length and effective range of the helium potential (see Appendix for details). This interaction indeed reproduces the scattering length dependence of the deviations, although the trimer energies are off by a small shift that can be removed by adding a threebody force [81]. Alternatively, one can use the finiterange corrected universal formula (4.42). This formula allows one to map the helium trimer energies to the ideal Efimov spectrum by plotting the renormalised wave number $\kappa^{\prime}=\lambda_{n} \kappa$ as a function of the renormalised inverse scattering length $1 / a^{\prime}=\lambda_{n} / a_{B}$, where $\lambda_{n}=\left(1+\Gamma_{n} /\left(\kappa_{*} a\right)\right)^{-1}$. The resulting plot is shown in figure 4.8 .

Experimental observations The first experimental investigation was carried out by the group of Jan Peter Toennies in Göttingen [27]. A beam of helium clusters was generated by cryogenic expansion and diffracted through a nanostructured transmission grating. The mass and spatial extent of diffracted clusters were measured, revealing the existence of the ground-state helium trimer with spatial extent of $\langle r\rangle=1.1_{-0.5}^{+0.4} \mathrm{~nm}$, in agreement with theoretical predictions. The excited trimer was not observed in that experiment. Both trimers were eventually observed in the group of Reinhard Dörner in Frankfurt [121, 12] by Coulomb explosion imaging. Although the trimer energies have not been measured, the Coulomb explosion imaging technique enables to measure structural properties of the trimers and to some extent reconstruct their wave functions, in very good agreement with the theoretical cal- 
culations. From these observations, the authors concluded that the ground trimer's structural properties are very different from those of an ideal Efimov trimer, whereas the excited trimer conforms relatively well to the structure of an Efimov trimer.

\subsection{2 ultra-cold atoms under Feshbach resonances}

The field of ultra-cold atomic gases has developed from laser-cooling experiments in the 1980s. It consists in magnetically or optically trapping inside a vacuum chamber a cloud of atoms cooled to ultra-low temperatures, from microkelvins down to a few nanokelvins. In this setting, it was realised that the scattering length between the atoms could be changed through a Feshbach resonance by applying a magnetic field to the cloud [28, 126].

A Feshbach resonance $[129,130,131]$ is a general resonance phenomenon of particles with different possible internal states (for instance, hyperfine states in the case of atoms). The various pair combinations of internal states constitute different two-body scattering channels. These channels are in general coupled at short distance by some interactions (for example, the hyperfine interaction in the case of atoms). If the scattering threshold of some incoming channel (the "open" channel) is close to the energy level of a twobody bound state in another or several other channels (the "closed" channel), the coupling between these two channels makes the scattering in the open channel resonant, i.e. the scattering length can become much larger than the range of the interaction potentials in these various channels. If the spacing between the threshold and bound-state energy can be controlled (for instance by applying a magnetic field and shifting by the Zeeman effect the threshold and bound-state energy by different amounts), the resonance condition and therefore the scattering length can be tuned. This technique has been extremely successful for controlling interactions in ultra-cold atomic gases, and studying Efimov physics in particular.

Observation through loss Atomic Efimov trimers arise when the scattering length of the interatomic potentials is tuned to a large value. This corresponds to the presence of an $s$-wave two-body bound state or virtual state near the two-body scattering threshold. However, for atoms commonly used in ultra-cold gases, these potentials also support many other twobody bound states that are more deeply bound, i.e. diatomic molecular states of various rotational symmetries. This introduces two complications with respect to the ideal Efimov scenario.

The first one is that the many diatomic molecular levels experience different Zeeman shifts as the magnetic field is varied to tune the scattering length. This can result in a second molecular state reaching the threshold and creating a Feshbach resonance overlaping with the resonance of interest, thus complicating the relationship between the magnetic field and the scattering length. This situation is illustrated by the many overlapping Feshbach resonances of caesium-133 ground-state atoms [132]. However, when the two resonances have very different widths, the Efimov physics associated with one resonance may not be significantly affected by the presence of the other. A second consequence of the different Zeeman shifts of the molecular levels is that there can be many avoided crossings between these levels. Sometimes such a crossing may strongly affect the $s$-wave two-body bound state associated with the Feshbach resonance, resulting in a quick departure from the zero-range picture. This is the case, for instance, for the caesium dimer associated with the Feshbach resonance near $800 \mathrm{G}$, which remains very close to the threshold due to an avoided crossing with a weakly bound molecular level of the open channel [119]. Nevertheless, the Efimov effect still occurs close to resonance.

The second complication is that Efimov trimers are not true bound states, since they have a much smaller binding energy than the many diatomic molecular states. Instead, these trimers exist as resonances embedded in the continua formed by the scattering of one atom and one diatomic molecule, as shown by the dotted curves in figure 4.3. As a result, these trimers have a finite lifetime as they can decay in these continua by dissociating into an atom and diatomic molecule. This situation is different from that of helium-4, for which the potential supports only one two-body bound state and Efimov trimers are true bound states that are infinitely long lived. Nevertheless, the dissociation processes are weak enough to allow to resolve the trimer resonances, which were found to conform to the general Efimov scenario. It is for this reason that these resonances are thought to be a good approximation of Efimov states, even though they are not true bound states.

Ultra-cold atom experiments typically start with a gas of unbound atoms, dimers, or atom-dimer mixtures. Three-body bound states can be formed through inelastic collisions [133] but these states are difficult to observe in the standard setups. Nevertheless, these collisions tend to deplete the gas of atoms and this loss can be monitored by imaging the gas. This can be used to indirectly observe the three-body bound states through their influence on inelastic collisions. For example, when three atoms collide in an ultra-cold gas, two of them may recombine into a diatomic molecule. The energy gained by this binding is then redistributed as kinetic energy between the formed molecule and third atom. This inelastic process can be strongly enhanced at the low collisional energy of ultra-cold atoms by Efimov resonances that occur whenever an Efimov trimer lies just below the three-body scattering threshold. As one varies the scattering length through the values $a_{-}$ shown in figure 4.2 , one therefore expects to see peaks in the loss rate. The existence of these peaks were first 
predicted in 1999 by Brett D. Esry, Chris H. Greene, and James P. Burke Jr in reference [31]. Eric Braaten and Hans-Werner Hammer subsequently derived from the zero-range theory a simple analytical formula for the three-body recombination loss rate coefficient $L_{3}$ at zero temperature $[134,135]$ :

$$
L_{3}=\frac{C \sinh (2 \eta)}{\sin ^{2}\left[\left|s_{0}\right| \ln \left(a / a_{-}\right)\right]+\sinh ^{2} \eta} \frac{\hbar a^{4}}{m},
$$

as a function of the scattering length $a$, the threebody parameter characterised by $a_{-}$, and an inelasticity parameter $\eta$ describing the decay to diatomic molecules. $C \approx 4590$ is a numerical constant. This formula shows that $L_{3}$ has a local maximum when the $\sin ^{2}$ term vanishes, corresponding to scattering lengths that are multiples of $a_{-}$with the Efimov scaling factor, i.e. $a=e^{\pi n /\left|s_{0}\right|} a_{-} \approx(22.7)^{n} a_{-}$.

Such a peak was seen in the early experiments on Feshbach resonances and subsequently interpreted as an Efimov resonance. The first observation was made in 2002 and the final results were reported in 2006 [32] by the group led by Hanns-Christoph Nägerl and Rudolf Grimm in Innsbruck for an ultra-cold gas of caesium-133 atoms in their hyperfine ground state $\left|F=3, m_{F}=3\right\rangle$, where $F$ and $m_{F}$ designate the hyperfine quantum numbers. The Feshbach resonance used to tune the scattering length in this experiment is a bit particular, because it occurs at a "negative value of the magnetic field" $B \approx-10 \mathrm{G}$, which physically corresponds to the excited hyperfine state $\mid F=3, m_{F}=$ $-3\rangle$ of caesium-133. This excited state is not stable against two-body decay, so the experiment was limited to the positive values of $B$. Although the dimer causing the resonance exists only for $B \leq-10 \mathrm{G}$, the resonance makes the scattering length very large and negative for $0 \leq B \lesssim 10 \mathrm{G}$, making it possible to observe Efimov physics. In this range, however, only the ground Efimov state exists [119], and was revealed by a peak in the measured three-body loss rate.

This allowed not only to prove the existence of an Efimov trimer, but also measure the value $a_{-}$which is related to Efimov's three-body parameter. Since the ground-state Efimov trimer does not completely follow the Efimov universal scenario, some objections on the terminology of "Efimov state" were raised [119]. Indeed, the caesium trimer evidenced in the experiment, when followed to the negative side of magnetic field values where the dimer appears from the two-body threshold, is not expected to approach the atom-dimer threshold, and therefore does not conform to definition 2 given in section 4.5. However, it is expected to be an Efimov state according to definition 3 and 4 . Moreover, unlike the ground-state trimer of helium-4, which occurs in the region of positive scattering length where deviations from the Efimov scenario are significant, the loss peak observed for caesium corresponds to the region of negative scattering length, where the groundstate trimer is borromean and follows more closely the
Efimov scenario. For these reasons, this experiment may be thought to constitute the first experimental demonstration of a borromean Efimov state.

In the following years, many similar observations of Efimov resonances were made with various kinds of atomic species. For positive scattering lengths, the trimer energy is below the dimer energy. At some value $a_{*}$ of the scattering length, the trimer energy can reach, or approach closely, the atom-dimer threshold, creating a low-energy resonance in atom-dimer scattering, which can be seen as a peak in the rate of losses associated with inelastic atom-dimer scattering. Efimov physics can also be seen in the three-body inelastic scattering for positive scattering length. In this case, recombination to dimers can take two paths, and as a result of quantum interference between the two paths, a minimum in the three-body recombination loss rate is expected for a value $a_{+}$of the scattering length ${ }^{9}$. The zero-range theory for these Efimov features in the loss measurement at zero temperature were done in several theoretical contributions $[134,135,11]$ (see references in [11]). The influence of temperature has appeared to play an important role for the identification and interpretation of the resonance position, and was treated theoretically in Refs. [136, 137].

Studying the inelastic collisions between weakly bound dimers and unbound atoms of caesium-133 [138], the Innsbruck group observed an atom-dimer resonance as predicted by the Efimov scenario and measured the value of $a_{*}$ of figure 4.2. The group of Giovanni Modugno and Massimo Inguscio in Florence [139] observed both a local maximum and local minima in the threebody recombination rate of potassium-39, thus determining the values of $a_{-}$and $a_{+}$, as well as inferring the atom-dimer resonance position $a_{*}$ from a small peak of the three-body loss rate appearing on the positive scattering length side. A similar experiment was performed for lithium-7 atoms by the group of Randall G. Hulet at Rice University [140]. From these experiments, the following measured ratios were reported: $a_{-} / a_{+}=-6.7 \pm 0.6\left[{ }^{39} \mathrm{~K}\right],-2.5 \pm 0.2\left[{ }^{7} \mathrm{Li}\right]$ and $a_{-} / a_{*}=-50 \pm 3\left[{ }^{39} \mathrm{~K}\right], 10.4 \pm 1.5\left[{ }^{7} \mathrm{Li}\right]$. These results were later modified in 2013 in Refs. [141] and [142] due to a misassignment of the three-body loss peak $^{10}$ and to a recalibration of the Feshbach resonance parameters, respectively. The updated values $a_{-} / a_{+}=-3.1 \pm 0.3\left[{ }^{39} \mathrm{~K}\right],-2.8 \pm 0.3\left[{ }^{7} \mathrm{Li}\right]$ and $a_{-} / a_{*}=-22 \pm 2\left[{ }^{7} \mathrm{Li}\right]$ are in fair agreement with the values predicted by the universal Efimov theory

\footnotetext{
${ }^{9}$ Such mininum was already seen in the original experiment of reference [32], although it is located at higher magnetic fields, accross a zero of the scattering length; it therefore corresponds to an adjacent Feshbach resonance, i.e. a different window of universality than that of the observed peak

${ }^{10}$ In reference[139], two peaks were found at $a=$ $-1500 a_{0},-650 a_{0}$, which were interpreted as signatures of an Efimov trimer $\left(a_{-}=-1500 a_{0}\right)$ and an associated tetramer. Later however, the same group could not find the peak at $a=-1500 a_{0}$ [141], and reassigned the other peak to a trimer, with the updated value $a_{-}=-690(40) a_{0}$.
} 
$a_{-} / a_{+} \approx-4.9$ and $a_{-} / a_{*} \approx-22.0$ [4]. The group of Lev Khaykovich [143] performed a similar experiment using lithium-7 atoms, and measured $a_{-}$and $a_{*}$. The ratio $a_{*} / a_{-}=-1.01(15)$ obtained in the experiment agrees with the theoretical value above if $a_{-}$and $a_{*}$ are interpreted as corresponding to the ground and first-excited Efimov trimers and the universal scaling factor 22.7 is multiplied. The groups from Florence [139] and Rice University [140] have both reported the observation of two dips in the loss rate for positive scattering lengths, i.e. two values of $a_{+}$corresponding to a ground-state and an excited Efimov trimers. They found the ratio between these two values to be 25(4) and 22.5(3) respectively, roughly confirming the discrete scale invariance of Efimov trimers with the universal ratio 22.7. The universal scaling was reported to be observed for negative scattering lengths as well in reference [140], although a subsequent recalibration of the scattering length with respect to the magnetic field dismissed the second value of $a_{-}$and corrected the values of $a_{+}$, giving a updated ratio 16(2) [142].

More recently, the second Efimov trimer could be observed for negative scattering lengths with caesium133 atoms near a 800 G Feshbash resonance [40], as originally proposed in reference [119]. The scaling factor between the ground state Efimov trimer and second Efimov trimer was found to be 21.0(1.3), close to the universal ratio 22.7. The experimental data for the two Efimov resonances are shown in figure 4.9. This is so far the most convincing experimental evidence of Efimov states of identical bosons, according to definition 1 of section 4.5 .

Bound-state spectroscopy Before the evidence of Efimov states through three-body and two-body losses, the association of three colliding atoms in an Efimov trimer was theoretically proposed [144]. Such an association, although different from the original proposal, could be achieved experimentally and allowed the direct spectroscopy of Efimov trimers. Although the first demonstration was done with three distinguishable atoms (see section 6.5), the association of three bosons into an Efimov trimer was later achieved with lithium-7 atoms in the group of Lev Khaykovich [145]. The experiment consists in driving a transition between three colliding atoms and the underlying Efimov trimer state by applying a radio-frequency (rf) modulation of the magnetic field. When the frequency of the modulation matches the energy difference between the energy of the colliding atoms and the trimer energy, the three atoms are associated in trimers, resulting in a loss of the atoms from the imaged cloud. The resonance could be seen as a small dip in the number of atoms as the frequency of the modulation is varied, on the shoulder of a broader dip due to the association of two atoms into a dimer.

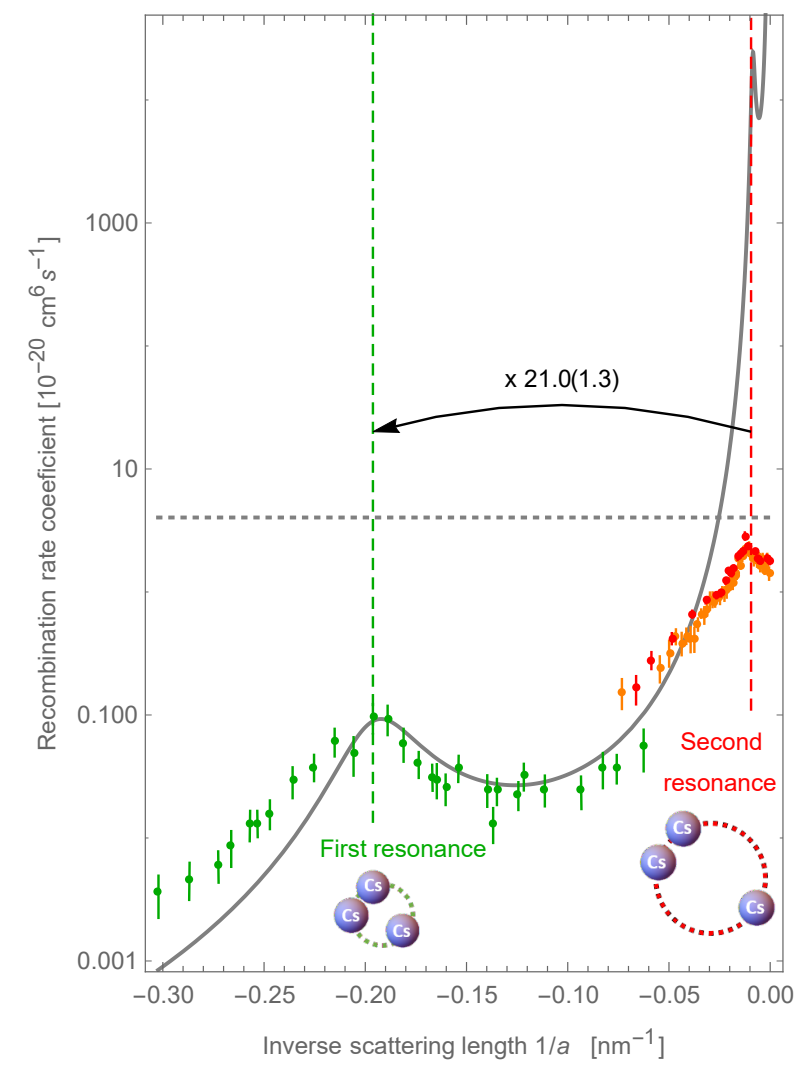

Figure 4.9: Efimov resonances in an ultra-cold gas of caesium-133 atoms in their lowest hyperfine state, whose scattering length is varied by applying a magnetic field on the order of 800 gauss (adapted from reference [40]). The resonances appear as peaks in the three-body recombination rate as a function of scattering length. The experimental data of reference [40] is shown with different colours corresponding to different data sets, all taken at a temperature of about $10 \mathrm{nK}$. The data around the first resonance is fitted by the zero-temperature universal formula (4.52) with $a_{-}=$ $-51.0(0.6) \mathrm{nm}$ and $\eta=0.10(1)$. The formula predicts a second resonance at $a=22.7 \times-51=-1158 \mathrm{~nm}$. The second resonance was observed at $a=-1068(63) \mathrm{nm}$, corresponding to a factor 21(1.3) indicated by the arrow. The height of the peak is much smaller than the zero-temperature prediction, due to the saturation effect of finite temperature [136, 137], indicated by the horizontal dotted line for $10 \mathrm{nK}$.

\subsection{Prospects for observation in condensed matter}

Most studies of Efimov physics have been done in the fields of nuclear physics and atomic physics, but recently there have been works seeking the possibility of Efimov physics in other physical systems. Here, we present the current prospects for quantum spin systems $[146,147]$ and excitons [148]. 


\subsubsection{Efimov states in quantum spin systems}

Quantum spin systems have constituted an important and active field of research in condensed matter physics for more than 70 years. They have been been used with some success to explain and predict various magnetic phenomena in insulating solids. A quantum spin system is a system of spins fixed on the sites of a lattice, which can interact with each other through an exchange interaction. Depending on the geometry of the lattice, spatial dimension, and the sign and the spatial extent of the exchange interaction, quantum spin systems can exhibit various magnetic phases.

When the ground state of a quantum spin system shows a non-trivial magnetic phase, such as the ferromagnetic or anti-ferromagnetic phases, the symmetry of the Hamiltonian is spontaneously broken. The Nambu-Goldstone theorem dictates that there must exist gapless low-energy excitations, called magnons, in such broken-symmetry systems. For a ferromagnet system, the magnons have the dispersion relation $\varepsilon(k) \propto k^{2}$ [149]. This dispersion is similar to that of a non-relativistic particle in the vacuum.

From this similarity between the magnons and the non-relativistic particles, Yusuke Nishida and coworkers [14] have sought the possibility of finding Efimov states in a ferromagetic quantum spin system. To achieve the resonant-interaction condition (4.4) necessary for the appearance of the Efimov states, they considered the Heisenberg model in three spatial dimensions with an anisotropic exchange coupling and the single-ion anisotropy. These terms originate from the anisotropy in the lattice and the crystal field effect that are present in real materials described by quantum spin systems. In the presence of these terms, the magnons no longer behave as independent quasi-particles, and start to interact. This interacting problem is greatly simplified from the fact that the number of magnons is a conserved quantity in the anisotropic Heisenberg model. It is therefore justified to consider the fewbody problem for magnons with a well-defined number of magnons. Y. Nishida and co-workers have shown by analytically solving the two-body problem of magnons that the magnons can form a bound state when the anisotropies are increased. As a result, the scattering length between the magnons diverges at some critical values of the anisotropies at which the bound state of magnons appears, a necessary condition for the onset of Efimov physics. Y. Nishida and co-workers have solved numerically the three-magnon problem at the resonant point. For different values of the spin quantum number and critical anisotropies, the three-body energy spectrum of magnons is found to be in good agreement with the universal Efimov spectrum, and to exhibit a discrete scale invariance with a scaling factor close to 22.7

It is important to note that the following features of the anisotropic Heisenberg model are the same as the resonantly interacting non-relativistic particles V. Efi- mov considers in his original argument: (a) magnons have a low-energy dispersion $\varepsilon(k) \propto k^{2}$, (b) the number of magnons is conserved, (c) the interaction between magnons is short-ranged. These sufficient conditions seem to be a useful guideline for searching Efimov states in other systems.

To achieve the Efimov states of magnons, one needs a fine tuning of the parameters of the system close to the resonantly interacting regime. In the above case, one needs to increase the anisotropy of the exchange interaction or the single-ion anisotropy term, such that the bound state of two magnons is about to appear. Although such a fine tuning is rather challenging, it is known that in organic crystals, the exchange coupling can be modified by applying an external pressure. This may be one route to realise Efimov states in quantum spin systems. Once one finds a suitable system, Y. Nishida and co-workers [14] have argued that the appearance of Efimov states can be tested experimentally from the electron spin resonance signal [146].

\subsubsection{Universal few-body physics with excitons}

An exciton is a bound state of an electron and a hole which appears in a semiconductor excited by a laser. Because it is an association of two fermions, it behaves as a boson. While there has been a long history of exciton studies since its first theoretical prediction [150, 151], there has been a revival of interest recently by the realisation of the Bose-Einstein condensates of the excitons [152], which had been one of the holy grails in the research on excitons.

Recently, a possible signature of Efimov physics in an excitonic system has been claimed to be observed [148]. In reference [148], $N$-body bound states of excitons, called poly-excitons, have been observed up to $N=6$ by a photoluminescence measurement in a diamond crystal. The binding energies of these poly-exciton states were measured, and compared with those found in other crystals $[153,154]$. They have been found to agree excellently between different crystals if normalized by the binding energy of an exciton, suggesting that the poly-exciton states behave universally, i.e., they are independent of the details of the crystal and the energy scale of the system. The binding energies are reminiscent of the series of universal binding energies for the $N$-body clusters of resonantly interacting bosons discussed in section 12.2, although it is not clear whether they are related since the exciton-exciton interaction is typically non-resonant.

\section{Three identical fermions}

Identical fermions (i.e., fermions in the same internal state) cannot interact in the $s$-wave channel due to the antisymmetrisation, so that the conventional Efimov physics appearing for the $s$-wave resonance (see section 4) does not occur for such Fermi systems. Two 
identical fermions can only scatter in the odd angular momentum channels, and among them, the most dominant channel at low energy is the $p$-wave channel. Therefore, the scattering amplitude at low energy is written as

$$
f(k, \theta)=\frac{k^{2} \cos \theta}{-1 / a_{p}+\frac{1}{2} r_{p} k^{2}-i k^{3}},
$$

where $a_{p}$ is the $p$-wave scattering volume, $r_{p}$ is the $p$ wave inverse effective range. Note that, although the notations are similar to those of equation (4.41), $a_{p}$ is a volume and $r_{p}$ is the inverse of a length. A natural step is to investigate universal three-body physics of identical fermions in the vicinity of resonant $p$-wave interaction, i.e., when the moduli of $p$-wave scattering volume becomes divergently large. Although such $p$-wave resonances are experimentally challenging because they are much narrower than the $s$-wave ones and subject to larger atomic number losses, they have been successfully realised in ultra-cold atom experiments with Feshbach resonances $[155,156,157,158]$. In this section, we review the recent theoretical progress on the fewfermion physics in the vicinity of a $p$-wave resonance in three dimensions (see 5.1), and two dimensions (see $5.2)$.

\subsection{Resonant $p$-wave interactions}

In three dimensions, the possibility of Efimov trimers has been investigated for three fermions with $p$-wave resonant interactions $[159,160,161]$. If one takes $1 / a_{p}=0$ and $r_{p}=0$ simultaneously in equation (5.1), the moduli of the scattering amplitude takes its maximum value allowed by the unitary bound and the system becomes scale invariant, similarly to the unitary limit in the $s$-wave case: $1 / a=0$ and $r_{e}=0$. In this unitary limit $1 / a_{p}=0$ and $r_{p}=0$, Macek and Sternberg have shown, using a pseudo-potential method, that the Efimov effect occurs for the identical fermions for a spin $1 / 2$ system (i.e., among three identical fermions, two are in the same internal state, while the other is in another internal state) [159]. The same conclusion was obtained in reference [160].

However, this unitary limit is unphysical, violating the positivity of probability $[160,161,162,163]$. Indeed, if one believes the $p$-wave form of the scattering amplitude in equation (5.1) for any $k<\Lambda$, where $\Lambda$ is a cutoff momentum $\Lambda \gg\left|a_{p}\right|^{-1 / 3},\left|r_{p}\right|$, then the probability of the bound-state wave function outside the range $b$ of the potential exceeds unity, suggesting a negative probability at short distance [160, 161, 162, 163]. The positivity of the probability thus dictates the inverse range $r_{p}$ to have a finite negative value, satisfying the Wigner bound $[164,165]$.

$$
r_{p}<-\frac{2}{b}
$$

Equivalently, the scattering amplitude equation (5.1) is valid only for $k \ll\left|r_{p}\right|$ at the $p$-wave resonance, with $r_{p}<0$ satisfying the Wigner bound.

In Refs. [162, 166], spin-polarised three identical fermions around the $p$-wave resonance have been studied, keeping the Wigner bound. Three-body bound states with threefold rotational degeneracy have been found, one state for each channel in $L=1^{-}$channel $[162,166]$ and in $L=1^{+}$channel [162]. Both three-body bound states have larger binding energies than that of the $p$-wave dimer around the $p$-wave resonance. This suggests that the $p$-wave molecular BEC phase predicted in the studies of the $p$-wave resonant two-component Fermi system [167, 168, 169] is not a genuine ground state, but can be subject to trimer formation instability. The recombination rate to the trimers in a gas of $p$-wave dimers has been estimated in Refs. [166, 170], while the three-body recombination to the $p$-wave dimer state in a gas of identical fermions has been studied in Refs. [162, 171]. The trimer states found here are Borromean states [162]: they have finite binding energies at the $p$-wave resonance $1 / a_{p}=0$, and they persist for $1 / a_{p}<0$, where no $p$-wave dimer exists. As one moves further away from the resonance towards the negative scattering volume side, the trimers finally dissociate into three fermions at three-body continuum $E=0$. On the positive scattering volume side, the trimer energy becomes equal to the dimer energy, so that the trimers dissociate into a particle plus a $p$ wave dimer.

\subsection{The super-Efimov effect}

In two dimensions, more exotic few-body states emerge. In reference [147], Yusuke Nishida and coworkers have found that there exists an infinite series of three-body bound states in $\ell= \pm 1$ channels with discrete scale invariance. These states, unlike the Efimov states, show a double exponential scaling of their energies

$$
E^{(n)}=E_{*} \exp \left[-2 \exp \left(\frac{3 \pi n}{4}+\theta\right)\right] .
$$

For this reason, they have been dubbed "super-Efimov states". Here, $\theta$ is a parameter determined by the shortrange part of the interaction (see the final paragraph of this section for more details). The super-Efimov states, in addition to a non-super-Efimovian $\ell=0$ ground state, have been found to be Borromean [172, 173], i.e., they remain bound even in the absence of a two-body $p$ wave dimer. We note that the three-fermion problem in two dimensions at the $p$-wave resonance was also solved in reference [170] before the super-Efimov states have been found by Yusuke Nishida and co-workers in reference [147], and the on-shell $T$-matrix at the threshold energy was found to show the same double exponential scaling behavior as equation (5.3).

While Yusuke Nishida and co-workers have found the super-Efimov states with the renormalization group 
analysis, as well as by solving a momentum-space integral equation for a separable potential [147], they can also be found by other formalisms [172, 173, 174, 175]. A rigorous mathematical study based on a spectral analysis has proved the presence of the super-Efimov effect [174]. The super-Efimov states can also be demonstrated by the hyper-spherical formalism, but some remarks are in order. In the hyper-spherical formalism, the diagonal adiabatic potential in the super-Efimovian channel $\ell= \pm 1$ is found to be $\left(V_{n}\right.$ here has the same notation as in equation (4.31)) [172, 175]

$$
\begin{aligned}
V_{0}(R)= & -\frac{1}{4 R^{2}}-\frac{Y}{R^{2} \ln \left(R / R_{0}\right)}-\frac{16 / 9}{R^{2} \ln ^{2}\left(R / R_{0}\right)} \\
& +O\left(\frac{1}{R^{2} \ln ^{3}\left(R / R_{0}\right)}\right)
\end{aligned}
$$

where $R_{0}$ is the range of the interaction, and $Y$ is a parameter dependent on the short-range part of the interaction. If $Y=0$ and one can neglect the second term, one arrives at the super-Efimov states with a double exponential scal$\operatorname{ing} E^{(n)} \propto \exp \left[-2 \exp \left(\pi n / \sqrt{\frac{16}{9}-\frac{1}{4}}\right)\right]$, which is slightly different from equation (5.3) [172]. However, $Y$ turns out to be positive definite $[172,173]$, and the second term is relevant at large $R$. Due to the second term, equation (5.4) leads to a different scaling behavior $E^{(n)} \propto \exp \left(-\frac{\pi^{2} n^{2}}{2 Y}\right)$ at low energy [175]. The discrepancy between these results can be ascribed to the non-adiabatic term $Q_{n m}(R)[172,173]$. The diagonal non-adiabatic term in the super-Efimovian channel $Q_{00}(R)$ has been found to behave in the leading order as $[172]$

$$
Q_{00}(R)=\frac{Y}{R^{2} \ln \left(R / R_{0}\right)}+O\left(\frac{1}{R^{2} \ln ^{2}\left(R / R_{0}\right)}\right) .
$$

One can see that the first term cancels the second term in equation (5.4). In reference [173], Chao Gao and co-authors have studied this system numerically for various classes of potentials, and the next leading order of $Q_{00}(R)$ has been found to be consistent with $-\frac{1}{4 R^{2} \ln ^{2}\left(R / R_{0}\right)}$. One thus obtains a correct hyperradial potential in the super-Efimovian channel as

$$
\begin{aligned}
V_{0}(R)+Q_{00}(R)= & -\frac{1}{4 R^{2}}-\frac{16 / 9+1 / 4}{R^{2} \ln ^{2}\left(R / R_{0}\right)} \\
& +O\left(\frac{1}{R^{2} \ln ^{3}\left(R / R_{0}\right)}\right),
\end{aligned}
$$

which reproduces the double exponential scaling of equation (5.3) found in reference [147].

While the double exponential scaling $\ln \left|E^{(n+1)}\right| / \ln \left|E^{(n)}\right|=\exp \left(\frac{3 \pi}{4}\right) \approx 10.6$ seems too large to be observed in a realistic system, it can be reduced significantly for mass-imbalanced two-component systems [176]. It has been found that the super-Efimov effect also occurs in a 2 identical fermions +1 particle system or 2 identical bosons +1 particle system when the inter-species $p$-wave interaction is resonant. The scaling factor of the super-Efimov states decreases as the inter-species mass ratio is increased towards a 2 heavy +1 light configuration. For instance, for a mixture of ${ }^{6} \mathrm{Li}$ and ${ }^{133} \mathrm{Cs}$ atoms, corresponding to the mass ratio 22.1 , it reaches a reasonable value $\ln \left|E^{(n+1)}\right| / \ln \left|E^{(n)}\right| \approx 1.3$. Note that the adiabatic approximation, i.e., the Born-Oppenheimer approximation, also fails for the mass-imbalanced system even for large mass ratios [176], leading to a similarly wrong energy spectrum as mentioned above [177].

Interestingly, Chao Gao and co-authors have found that the three-body parameters $E_{*}$ and $\theta$ are universal for the class of van der Waals potentials [173], in similarity with the van der Waals universality of the three-body parameter found for the Efimov states of three identical bosons (see section 11.2). This similarity is reinforced by the fact that four-body bound states ( $\ell= \pm 2$ channels) exist and are tied to each super-Efimov states, showing the same double exponential scaling, in the same way four-body bound states are associated with the Efimov trimers (see section 12). Although the super-Efimov trimers have yet to be observed in experiments, it is of interest to investigate the universality of the super-Efimov states in the $N$ body sector $(N \geq 4)$ and understand differences and similarities with the conventional Efimov physics for three bosons in three dimensions. We also note that a Borromean three-body bound state, possibly related to the super-Efimov states, has also been found in a two dimensional Hubbard model in the $S=3 / 2$ channel, which corresponds to a spin-polarised three-fermion system [178]. It is an interesting avenue to search for super-Efimov physics in such condensed-matter systems.

\section{Multi-component systems}

Systems with different kinds of particles, or particles with different internal states (either referred to as "components") exhibit an even richer Efimov physics than systems of identical particles. These systems have more parameters: the different kinds of particles may have different masses, quantum statistics, and different interactions between them. This situation introduces a few general facts:

- For a given three-particle system, there are three inter-particle interactions. At least two of these interactions should be resonant for the Efimov effect to occur. This can be understood simply from the picture of mediated interaction: in order for one particle to mediate an effective long-range interaction between two other particles, it must interact 
resonantly with these two particles. If it interacts resonantly with only one particle, then the mediation to another particle is not possible.

- Generally speaking, bosonic particles are favourable to the Efimov effect, whereas fermionic particles tend to prevent the Efimov effect, since their Pauli exclusion may overcome the Efimov attraction.

- The lighter a particle is, the better it mediates interaction between other particles. Thus, mass-imbalanced systems tend to enhance the Efimov attraction, and enable the Efimov effect in fermionic systems.

In the following, we review various situations.

\subsection{Three distinguishable particles}

The general treatment of three different kinds of particles with different masses and interacting with different scattering lengths was first addressed by Amado and Noble [22] and Efimov [20, 21]. Here, we consider the simpler case of three different particles with equal masses but different scattering lengths $a_{12}, a_{23}, a_{31}$. A concrete example of this situation is given by particles of the same kind, polarised in three different internal states - see section 6.3.2.

The general form of the Faddeev decomposition used in equation (4.16) involves three different Faddeev components:

$\Psi(1,2,3)=\chi^{(1)}\left(\vec{r}_{23}, \vec{\rho}_{23,1}\right)+\chi^{(2)}\left(\vec{r}_{31}, \vec{\rho}_{31,2}\right)+\chi^{(3)}\left(\vec{r}_{12}, \vec{\rho}_{12}\right.$

Following the derivation of section 4.1, we apply the Bethe-Peierls condition (4.7) for each pair and obtain:

$\frac{\partial \chi_{0}^{(i)}}{\partial \alpha}(R, 0)+\frac{4}{\sqrt{3}}\left(\chi_{0}^{(j)}\left(R, \frac{\pi}{3}\right)+\chi_{0}^{(k)}\left(R, \frac{\pi}{3}\right)\right)=-\frac{R}{a_{j k}} \chi_{0}^{(i)}(R, 0)$

Using the expansion $\chi_{0}^{(i)}(R, \alpha)=\sum_{n} F_{n}^{(i)}(R) \phi_{n}^{(i)}(\alpha ; R)$ where $\phi_{n}^{(i)}(\alpha ; R)$ has the form:

$$
\phi_{n}^{(i)}(\alpha ; R)=\sin \left(s_{n}(R)\left(\frac{\pi}{2}-\alpha\right)\right)
$$

the conditions can be written in a matrix form [4]:

$$
\begin{array}{r}
{\left[-\cos \left(s_{n} \frac{\pi}{2}\right)\left(\begin{array}{ccc}
1 & 0 & 0 \\
0 & 1 & 0 \\
0 & 0 & 1
\end{array}\right)+\frac{4}{\sqrt{3}} \frac{\sin \left(s_{n} \frac{\pi}{6}\right)}{s_{n}}\left(\begin{array}{lll}
0 & 1 & 1 \\
1 & 0 & 1 \\
1 & 1 & 0
\end{array}\right)\right.} \\
\left.+\frac{\sin \left(s_{n} \frac{\pi}{2}\right)}{s_{n}} R\left(\begin{array}{ccc}
a_{23}^{-1} & 0 & 0 \\
0 & a_{31}^{-1} & 0 \\
0 & 0 & a_{12}^{-1}
\end{array}\right)\right] \cdot\left(\begin{array}{l}
F_{n}^{(1)} \\
F_{n}^{(2)} \\
F_{n}^{(3)}
\end{array}\right)=0
\end{array}
$$

To obtain a non-trivial solution $F_{n}^{(i)} \neq 0$, the determinant of the matrix should be zero.

\subsubsection{Three resonantly interacting pairs}

For three resonantly large scattering lengths $\left|a_{i j}\right| \gg b$ and hyper-radius $b \ll R \ll\left|a_{i j}\right|$, the terms $R a_{i j}^{-1}$ can be neglected and the determinant becomes:

$\left(\cos \left(s_{n} \frac{\pi}{2}\right)+\frac{4}{\sqrt{3}} \frac{\sin \left(s_{n} \frac{\pi}{6}\right)}{s_{n}}\right)^{2}\left(\cos \left(s_{n} \frac{\pi}{2}\right)-\frac{8}{\sqrt{3}} \frac{\sin \left(s_{n} \frac{\pi}{6}\right)}{s_{n}}\right)$

whose second factor admits the same imaginary root $s_{0}$ as that of equation (4.29) for three identical bosons. This could be anticipated from the fact that the form of the wave function in equation (6.1) can reduce to the bosonic case of equation (4.16) with $\chi^{(i)}=\chi$, when all scattering lengths are equally large. Therefore, the same Efimov effect that occurs for three bosons also occurs for three distinguishable particles. In particular, this systems exhibits discrete scaling invariance with the same scaling ratio $e^{\pi /\left|s_{0}\right|} \approx 22.7$ at unitarity $a_{12}=a_{23}=a_{31}= \pm \infty$. As in the bosonic case, the discrete scaling invariance persists away from unitarity. However, it requires the simultaneous scaling of all scattering lengths. It may therefore not be apparent if only one or two scattering lengths are scaled.

\subsubsection{Two resonantly interacting pairs}

We now consider only two resonantly large scattering length, say $\left|a_{23}\right|,\left|a_{31}\right| \gg b$, and one non-resonant scattering length $a_{12}$ on the order of the interaction range $b$. In the region of hyper-radius $b \ll R \ll\left|a_{23}\right|,\left|a_{31}\right|$, the terms $R a_{23}^{-1}, R a_{31}^{-1} \sim 0$ in equation (6.2) can be neglected, whereas the term $R a_{12}^{-1}$ is large and imposes $F_{n}^{(3)} \approx 0$. We are thus left with the equations for $F_{n}^{(1)}$ and $F_{n}^{(2)}$. The determinant of the corresponding matrix is

$\left(\cos \left(s_{n} \frac{\pi}{2}\right)+\frac{4}{\sqrt{3}} \frac{\sin \left(s_{n} \frac{\pi}{6}\right)}{s_{n}}\right)\left(\cos \left(s_{n} \frac{\pi}{2}\right)-\frac{4}{\sqrt{3}} \frac{\sin \left(s_{n} \frac{\pi}{6}\right)}{s_{n}}\right)$, whose second factor admits an imaginary root $s_{0} \approx$ i0.4137. The Efimov effect therefore occurs in this case as well, although it is weaker. The corresponding discrete scaling invariance ratio is $e^{\pi /\left|s_{0}\right|} \approx 1986.12$, implying a very sparse Efimov spectrum.

\subsubsection{One resonantly interacting pair}

Finally, if there is only one resonantly large scattering length, say $a_{23}$, only $F_{n}^{(1)}$ contributes in equation (6.2) and one is left with:

$$
\cos \left(s_{n} \frac{\pi}{2}\right)=0
$$

which admits only real roots $s_{n}$. There is therefore no Efimov effect in this case. This is consistent with the physical picture that the Efimov effect is due to the exchange of a particle resonantly interacting with two other particles, which is not possible if only one pair is resonantly interacting. 


\subsection{Identical particles +1 particle}

We now consider the case when two of the three particles are identical, either identical bosons or identical fermions. Here, "identical particles" means that they are in the same internal states (for a treatment of identical particles with different internal states, see section 6.3). The mass $M$ of these two particles is in general different from the mass $m$ of the third particle.

The two identical particles are located at $\vec{x}_{2}$ and $\vec{x}_{3}$ and the light particle at $\vec{x}_{1}$. The three sets of Jacobi coordinates for this system read:

$$
\begin{aligned}
\vec{r}_{1 i} & =\vec{x}_{i}-\vec{x}_{1} \\
\vec{\rho}_{1 i, j} & =\frac{1}{\cos \gamma}\left(\vec{x}_{j}-\frac{M \vec{x}_{i}+m \vec{x}_{1}}{M+m}\right)
\end{aligned}
$$

for $\{i, j\}=\{1,2\}$, and

$$
\begin{aligned}
\vec{r}_{23} & =\frac{1}{2 \sin \gamma^{\prime}}\left(\vec{x}_{3}-\vec{x}_{2}\right) \\
\vec{\rho}_{23,1} & =\frac{1}{\cos \gamma^{\prime}}\left(\vec{x}_{1}-\frac{\vec{x}_{2}+\vec{x}_{3}}{2}\right),
\end{aligned}
$$

where the angles $\gamma \in[0, \pi / 2]$ and $\gamma^{\prime} \in[0, \pi / 4]$ are defined by:

$$
\begin{aligned}
\gamma & =\arcsin \frac{M}{M+m} \\
\gamma^{\prime} & =\arcsin \sqrt{\frac{m}{2(M+m)}}
\end{aligned}
$$

The three sets are shown in figure 6.1. They all satisfy $r_{i j}^{2}+\rho_{i j, k}^{2}=R^{2}$, where $R$ is the hyper-radius, and they are related to each other by the following rotation transformations:

$$
\begin{aligned}
\vec{r}_{13} & =\sin \gamma \vec{r}_{12}+\cos \gamma \vec{\rho}_{12,3} \\
\vec{\rho}_{13,2} & =\cos \gamma \vec{r}_{12}-\sin \gamma \vec{\rho}_{12,3} \\
\vec{r}_{32} & =\sin \gamma^{\prime} \vec{r}_{12}-\cos \gamma^{\prime} \vec{\rho}_{12,3} \\
\vec{\rho}_{32,1} & =-\cos \gamma^{\prime} \vec{r}_{12}-\sin \gamma^{\prime} \vec{\rho}_{12,3}
\end{aligned}
$$

Using the general Faddeev decomposition of equation (6.1), and taking into account the symmetry or antisymmetry of the wave function under the exchange of the identical particles 2 and 3, one obtains the following form of the wave function:

$$
\begin{aligned}
\Psi(1,2,3)= & \chi^{(1)}\left(\vec{r}_{32}, \vec{\rho}_{32,1}\right) \\
& +\chi^{(2)}\left(\vec{r}_{13}, \vec{\rho}_{13,2}\right) \pm \chi^{(2)}\left(\vec{r}_{12}, \vec{\rho}_{12,3}\right)
\end{aligned}
$$

The sign \pm corresponds to a plus sign in the case of identical bosons, and to a minus sign in the case of identical fermions.

Following the derivation of section 4.1, we apply the Bethe-Peierls boundary condition (4.7) for pair 12 and
32, and obtain:

$$
\begin{array}{r}
{\left[\left(\frac{\partial}{\partial r}+\frac{1}{a_{12}}\right) \chi_{0}^{(2)}(\vec{r}, \vec{\rho})\right]_{r \rightarrow 0} \pm 2 \frac{\chi_{0}^{(2)}(\cos \gamma \vec{\rho},-\sin \gamma \vec{\rho})}{\sin 2 \gamma \rho}} \\
+2 \frac{\chi_{0}^{(1)}\left(-\cos \gamma^{\prime} \vec{\rho},-\sin \gamma^{\prime} \vec{\rho}\right)}{\sin 2 \gamma^{\prime} \rho}=0 \\
{\left[\left(\frac{\partial}{\partial r}+\frac{1}{a_{32}}\right) \chi_{0}^{(1)}(\vec{r}, \vec{\rho})\right]_{r \rightarrow 0}+2 \frac{\chi_{0}^{(2)}\left(-\cos \gamma^{\prime} \vec{\rho},-\sin \gamma^{\prime} \vec{\rho}\right)}{\sin 2 \gamma^{\prime} \rho}} \\
\pm 2 \frac{\chi_{0}^{(2)}\left(-\cos \gamma^{\prime} \vec{\rho},-\sin \gamma^{\prime} \vec{\rho}\right)}{\sin 2 \gamma^{\prime} \rho}=0
\end{array}
$$

where $\chi_{0}^{(k)}(\vec{r}, \vec{\rho})=\chi^{(k)}(\vec{r}, \vec{\rho}) /(r \rho)$. The Faddeev components can be expressed as a function of the hyperspherical coordinates,

$$
\begin{aligned}
R & =\sqrt{r_{i j}^{2}+\rho_{i j, k}^{2}}, \\
\alpha_{k} & =\arctan \frac{r_{i j}}{\rho_{i j, k}},
\end{aligned}
$$

and the orientations $\hat{r}_{i j}$ and $\hat{\rho}_{i j, k}$ of $\vec{r}_{i j}$ and $\vec{\rho}_{i j, k}$. Since the particles interact in the $s$ wave, we consider the case when $\chi_{0}^{(k)}$ is independent of the orientation $\hat{r}_{i j}$. In these new coordinates, the Bethe-Peierls boundary conditions (6.3) become:

$$
\begin{array}{r}
{\left[\left(\frac{\partial}{\partial \alpha}+\frac{R}{a_{12}}\right) \chi_{0}^{(2)}(R, \alpha, \hat{\rho})\right]_{\alpha \rightarrow 0} \pm 2 \frac{\chi_{0}^{(2)}\left(R, \frac{\pi}{2}-\gamma,-\hat{\rho}\right)}{\sin 2 \gamma}} \\
+2 \frac{\chi_{0}^{(1)}\left(R, \frac{\pi}{2}-\gamma^{\prime},-\hat{\rho}\right)}{\sin 2 \gamma^{\prime}}=0 \\
{\left[\left(\frac{\partial}{\partial \alpha}+\frac{R}{a_{32}}\right) \chi_{0}^{(1)}(R, \alpha, \hat{\rho})\right]_{\alpha \rightarrow 0}+2 \frac{\chi_{0}^{(2)}\left(R, \frac{\pi}{2}-\gamma^{\prime},-\hat{\rho}\right)}{\sin 2 \gamma^{\prime}}} \\
\pm 2 \frac{\chi^{(2)}\left(R, \frac{\pi}{2}-\gamma^{\prime},-\hat{\rho}\right)}{\sin 2 \gamma^{\prime}}=0
\end{array}
$$

One can then expand $\chi_{0}^{(k)}$ as follows:

$$
\chi_{0}^{(k)}(R, \alpha, \hat{\rho})=\sum_{n, \ell, m} F_{n, \ell, m}^{(k)}(R) \phi_{n, \ell}^{(k)}(\alpha ; R) Y_{\ell m}(\hat{\rho})
$$

where $Y_{\ell m}$ are the spherical harmonics, and $\phi_{n, \ell}$ are the solutions of the eigenvector equation:

$$
\left(-\frac{\partial^{2}}{\partial \alpha^{2}}+\frac{\ell(\ell+1)}{\cos ^{2} \alpha}\right) \phi_{n, \ell}^{(k)}(\alpha ; R)=s_{n, \ell}^{2}(R) \phi_{n, \ell}^{(k)}(\alpha ; R)
$$

with the boundary conditions $\phi_{n, \ell}^{(i)}\left(\frac{\pi}{2}\right)=0$ and the Bethe-Peierls boundary conditions from equation (6.4). The eigenvalues $s_{n, \ell}^{(k)}(R)$ and eigenvectors $\phi_{n, \ell}(\alpha ; R)$ determine a set coupled of coupled equations satisfied by $F_{n, \ell, m}^{(k)}(R)$, from which the three-body problem can be solved. 

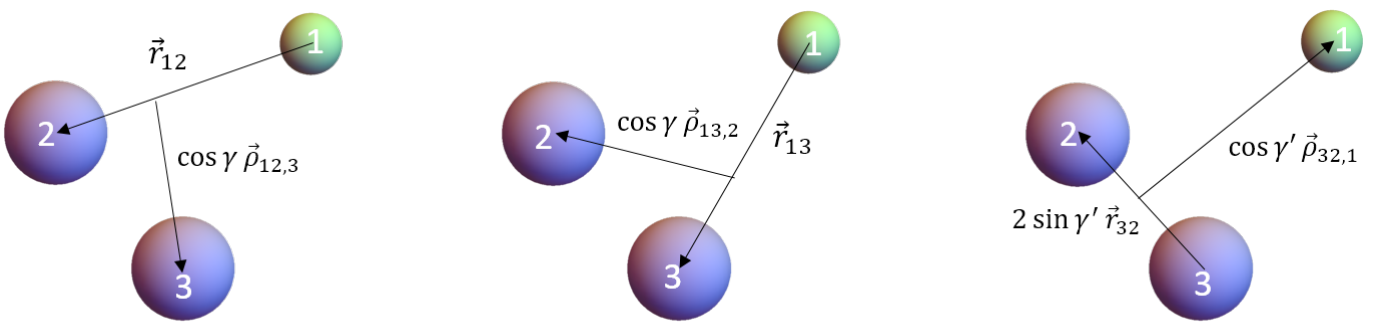

Figure 6.1: The three sets of Jacobi coordinates for two identical particles (dark) plus one particle (pale).

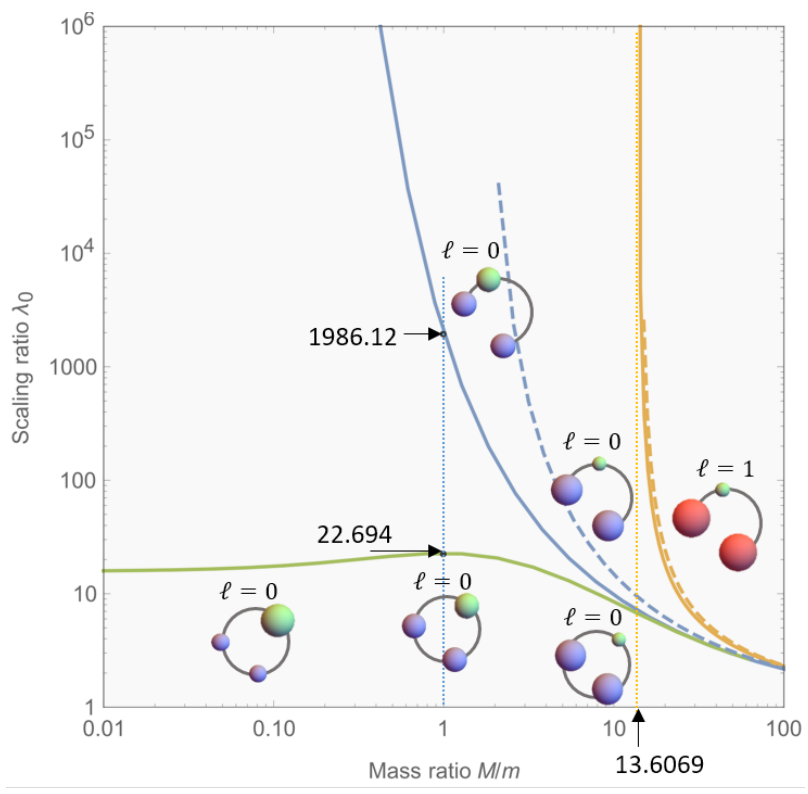

Figure 6.2: Efimov scaling ratio $\lambda_{0}=e^{\pi /\left|s_{0}\right|}$ as a function of the mass ratio for Efimov states of two identical particles and another particle. The lower curve (green) corresponds to two identical bosons and one particle resonantly interacting with each other. The middle curve (blue) corresponds to two identical bosons interacting resonantly only with the other particle. The upper curve (orange) corresponds to two identical fermions resonantly interacting with another particle. The dashed curves show the results obtained from the Born-Oppenheimer approximation for large mass ratios.

\subsubsection{2 bosons +1 particle with $\ell=0$}

If the two identical particles are bosonic, then $\pm=+$. For $\ell=0$, the solutions of equation (6.5) are given by $\phi_{n, 0}^{(k)}(\alpha ; R)=\sin \left(s_{n}\left(\frac{\pi}{2}-\alpha\right)\right)$. The conditions resulting from equation (6.4) can be written in a matrix form

$$
\begin{array}{r}
{\left[-\cos \left(s_{n} \frac{\pi}{2}\right)\left(\begin{array}{ll}
1 & 0 \\
0 & 1
\end{array}\right)+\frac{2}{s_{n}}\left(\begin{array}{cc}
\frac{\sin \left(s_{n} \gamma\right)}{\sin 2 \gamma} & \frac{\sin \left(s_{n} \gamma^{\prime}\right)}{\sin 2 \gamma^{\prime}} \\
2 \frac{\sin \left(s_{n} \gamma^{\prime}\right)}{\sin 2 \gamma^{\prime}} & 0
\end{array}\right)\right.} \\
\left.+\frac{\sin \left(s_{n} \frac{\pi}{2}\right)}{s_{n}} R\left(\begin{array}{cc}
a_{12}^{-1} & 0 \\
0 & a_{32}^{-1}
\end{array}\right)\right] \cdot\left(\begin{array}{l}
F_{n}^{(2)} \\
F_{n}^{(1)}
\end{array}\right)=0
\end{array}
$$

Three resonantly-interacting pairs We first consider the case when all scattering lengths are resonant, $\left|a_{12}\right|,\left|a_{32}\right| \gg b$. In the region of hyper-radius $b \ll$ $R \ll\left|a_{12}\right|,\left|a_{32}\right|$, the terms $R a_{12}^{-1}, R a_{32}^{-1} \sim 0$ in equation (6.6) can be neglected, and the determinant of the matrix in that equation is:

$$
\left(\cos \left(s_{n} \frac{\pi}{2}\right)-\frac{2}{s_{n}} \frac{\sin \left(s_{n} \gamma\right)}{\sin 2 \gamma}\right) \cos \left(s_{n} \frac{\pi}{2}\right)-2\left(\frac{2}{s_{n}} \frac{\sin \left(s_{n} \gamma^{\prime}\right)}{\sin 2 \gamma^{\prime}}\right)^{2} .
$$

As in the case of three identical particles, this determinant admits an imaginary root $s_{0}$. Hence, the Efimov effect occurs in this case, and the scaling ratio between Efimov states at unitarity $\left(a_{12}, a_{32} \rightarrow \pm \infty\right)$ is shown in figure 6.2. In the case of equal mass $M=m$, one retrieves the scaling ratio $e^{\pi /\left|s_{0}\right|} \approx 22.7$ obtained for three identical bosons. This ratio is decreased by either decreasing the mass ratio $M / m$ (it tends to 15.74 in the limit $M / m \rightarrow 0$ ) or increasing the mass ratio (it tends to 1 in the limit $M / m \rightarrow \infty$ ).

Two resonantly-interacting pairs We now consider the case when only the interaction between the particles of mass $M$ and mass $m$ is resonant, $\left|a_{12}\right| \gg b$, while the interaction between identical particles is nonresonant, $\left|a_{32}\right| \sim b$. In the region of hyper-radius $b \ll R \ll\left|a_{12}\right|$, the term $R a_{12}^{-1}$ in equation (6.6) may be neglected, whereas the term $R a_{32}^{-1}$ is very large and imposes $F_{n}^{(1)} \approx 0{ }^{11}$. The remaining condition reads:

$$
-\cos \left(s_{n} \frac{\pi}{2}\right)+\frac{2}{s_{n}} \frac{\sin \left(s_{n} \gamma\right)}{\sin 2 \gamma}=0,
$$

\footnotetext{
${ }^{11}$ The corrections due to the non-zero scattering length $a_{32}$ and the effective range between the non-identical particles were recently addressed in the framework of effective-field theory by Bijaya Acharya and co-workers [179]. It was shown that both corrections can be accounted for by a single additional threebody parameter.
} 
which admits one imaginary solution $s_{0}$. The Efimov effect occurs in this case too, and the scaling ratio between Efimov states at unitarity $\left(a_{12} \rightarrow \pm \infty\right)$ is shown in figure 6.2. Since there are only two resonant pairs, the Efimov attraction is weaker than for three resonant pairs, and for equal masses $M=m$, one retrieves the scaling ratio $e^{\pi /\left|s_{0}\right|} \approx 1986.12$ obtained in section 6.1 .

For large mass ratios, the scaling ratio of trimer energies becomes the same as in the case of three-resonantly interacting pairs. This large-mass-ratio limit is interesting because the trimer spectrum is denser than that of identical bosons, allowing to more easily observe several Efimov trimers. These trimers may be evidenced from the change in particle-dimer scattering length and relaxation rate, or three-body recombination rate [180, 181, 182].

\subsubsection{2 fermions +1 particle with $\ell=1$}

If the two identical particles are fermionic $( \pm=-)$, there cannot be any $s$-wave interaction between the two, which can be seen from the boundary condition (6.3), which imposes $\phi^{(1)}=0$. For $\ell=0$, the solutions of equation (6.5) are given by $\phi_{n, 0}^{(2)}(\alpha ; R)=$ $\sin \left(s_{n}\left(\frac{\pi}{2}-\alpha\right)\right)$, with the following condition resulting from equation (6.4),

$$
-\cos \left(s_{n} \frac{\pi}{2}\right)-\frac{2}{s_{n}} \frac{\sin \left(s_{n} \gamma\right)}{\sin 2 \gamma}=0
$$

This equation only admits real solutions, and thus there is no Efimov attraction in this case.

For $\ell=1$, however, solutions of equation (6.5) are given by [183]

$\phi_{n, 0}^{(2)}(\alpha ; R)=s_{n} \cos \left(s_{n}\left(\frac{\pi}{2}-\alpha\right)\right)-\tan \alpha \sin \left(s_{n}\left(\frac{\pi}{2}-\alpha\right)\right)$

with the following condition resulting from equation (6.4)

$$
\frac{1-s_{n}^{2}}{s_{n}} \tan \left(s_{n} \frac{\pi}{2}\right)-\frac{2 \cos \left(s_{n} \gamma\right)}{\sin 2 \gamma \cos \left(s_{n} \frac{\pi}{2}\right)}-\frac{\sin \left(s_{n} \gamma\right) / s_{n}}{\sin ^{2} \gamma \cos \left(s_{n} \frac{\pi}{2}\right)}
$$

This condition admits one imaginary solution $s_{0}$ for a mass ratio $\frac{M}{m}>\kappa_{c}$, where the critical mass ratio $\kappa_{c} \approx 13.6069657$. Although there is no Efimov effect for mass ratios smaller than $\kappa_{c}$ (in particular no Efimov effect in the equal-mass case), it occurs for mass ratios larger than the critical mass ratio $\kappa_{c}$. The corresponding scaling ratio at unitarity $\left(a_{12}=a_{13} \rightarrow \pm \infty\right)$ is shown in figure 6.2. It is infinitely large at the critical mass ratio and rapidly decreases to approach the scaling ratio for two bosons and one particle as the mass ratio is increased.

\subsubsection{Trimers with higher-angular momenta}

From the preceding discussion, it appears that there is in general a competition between the Efimov attraction and the centrifugal repulsion due to the angular momentum $\ell$. For large enough mass ratios, the Efimov attraction can overcome the centrifugal repulsion, gradually allowing the binding of Efimov trimers with higher angular momentum [21, 184, 185, 186, 187]. Using the same approach as in the preceding discussion, one can determine from equation (6.4) the critical mass ratios for the appearance of Efimov trimers of increasing angular momenta. In the case of bosons, trimers with even angular momentum appear at the following critical mass ratios $[188,186]$ :

$$
\begin{gathered}
\ell=2, \quad \kappa>38.630 \ldots \\
\ell=4, \quad \kappa>125.765 \ldots \\
\ldots
\end{gathered}
$$

In the case of fermions, trimers with odd angular momentum appear at the following mass ratios:

$$
\begin{gathered}
\ell=1, \quad \kappa>13.607 \ldots \\
\ell=3, \quad \kappa>75.994 \ldots \\
\ldots
\end{gathered}
$$

\subsubsection{The Born-Oppenheimer picture}

In all three cases presented above and shown in figure 6.2, the Efimov effect is strengthened as the mass ratio $M / m$ is increased. This can be simply understood from the Born-Oppenheimer approximation [189]. This approximation exploits the fact that $M$ is much larger than $m$ in this limit: the heavy particles of mass $M$ can thus be treated as slow particles, and the particle of mass $m$ as a fast particle.

The Born-Oppenheimer approximation consists in first solving the motion of the fast particle, for fixed positions of the heavy particles. Let us call $\vec{R}$ the relative position between the two heavy particles, and $\vec{r}$ the relative position between their centre of mass and the light particle. The three-body wave function is approximated by the form:

$$
\Psi(\vec{R}, \vec{r})=F(\vec{R}) \phi(\vec{r} ; \vec{R})
$$

where $\phi(\vec{r} ; \vec{R})$ is a solution of the problem for the light particle in presence of the heavy particles at a fixed separation $\vec{R}$. In the zero-range theory, this solution is a free wave satisfying

$$
-\frac{\hbar^{2}}{2 m} \nabla_{r}^{2} \phi(\vec{r} ; \vec{R})=\epsilon \phi(\vec{r} ; \vec{R})
$$

with the Bethe-Peierls boundary condition (4.7) between the heavy and light particles. The solution with the lowest energy $\epsilon<0$ is obtained with the following linear combination of two free waves originating from two sources at the locations of the heavy particles:

$$
\phi(\vec{r} ; \vec{R})=\frac{\exp (-\kappa|\vec{r}-\vec{R} / 2|)}{|\vec{r}-\vec{R} / 2|}+\frac{\exp (-\kappa|\vec{r}+\vec{R} / 2|)}{|\vec{r}+\vec{R} / 2|}
$$




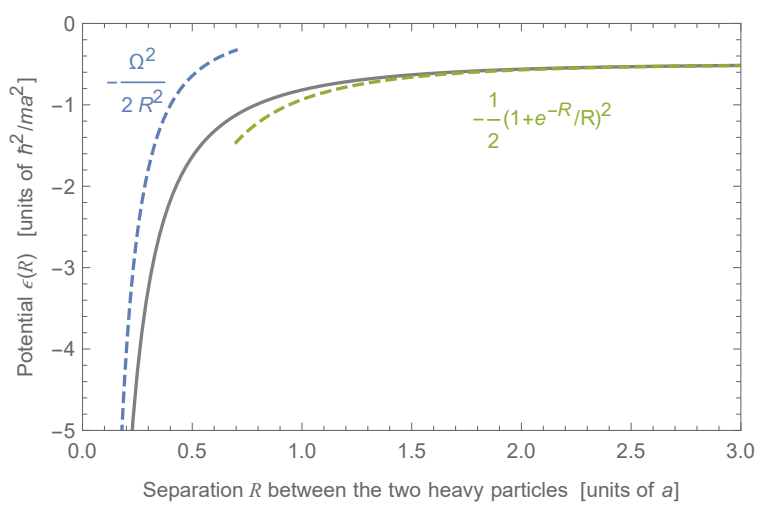

Figure 6.3: Bonding potential $\epsilon(R)$ in the BornOppenheimer approximation between two heavy particles separated by $R$ and both resonantly interacting with a light particle, with scattering length $a>0$. At separation much smaller than the scattering length $a$ between the heavy and light particles, the heavy particles experience an Efimov $R^{-2}$ attraction.

where $\kappa=\frac{1}{\hbar} \sqrt{-2 m \epsilon}$. Applying the Bethe-Peierls boundary condition (4.7) to equation (6.10), one gets an equation for $\kappa$ :

$$
\kappa-\frac{e^{-\kappa R}}{R}=\frac{1}{a} .
$$

The wave number $\kappa$, and thus the energy $\epsilon$, are therefore functions of the separation $R=|\vec{R}|$ between the two heavy particles. The $R$-dependent energy $\epsilon(R)$ constitutes a potential energy for the relative motion of the two heavy particles, which obeys the following Schrödinger equation,

$$
\left(-\frac{\hbar^{2}}{M} \nabla_{R}^{2}+\epsilon(R)\right) F(\vec{R})=E F(\vec{R}) .
$$

In addition to the potential $\epsilon(R)$ induced by the light particle, there should also be a short-range interaction potential between the two heavy particles, but it is neglected here. The potential energy $\epsilon(R)$ can be calculated analytically from equation (6.11) using the Lambert function and is shown in figure 6.3 for a positive scattering length. It is an attractive potential, whereas the potentials obtained for larger eigenvalues $\epsilon$ of equation (6.9) are repulsive. For this reason, the lowest-eigenvalue solution given by equation (6.10) is called a bonding orbital, to reflect the fact that the light particle in such a state acts as a glue between the two heavy particles.

For large $R \gg a$, one finds from equation (6.11) that

$$
\kappa \sim \frac{1}{a}+\frac{e^{-R / a}}{R},
$$

showing the bonding potential $\epsilon(R)$ asymptotes to the energy $-\frac{\hbar^{2}}{2 m a^{2}}$ corresponding to a two-body bound state of light and heavy particles scattering with a free heavy particle at zero energy. Moreover, the tail of the potential is as an attractive Yukawa potential whose range is the scattering length $a$.

For small $R \ll a$, on the other hand, one finds from equation (6.11) that $\kappa R$ approaches the Omega constant $\Omega \approx 0.567143$, solution of the equation $\Omega=e^{-\Omega}$. The bonding potential is therefore $\epsilon(R) \sim-\frac{\hbar^{2}}{2 m} \frac{\Omega^{2}}{R^{2}}$. This reproduces the $1 / R^{2}$ Efimov attraction. Hence, the Born-Oppenheimer approximation shows that the Efimov attraction can indeed be interpreted as resulting from the exchange of the light particle between the two heavy particles. Moreover, since $\epsilon(R)$ is proportional to $1 / m$ and the relative kinetic energy of the two heavy particles is proportional to $1 / M$, one can see from equation (6.12) that the Efimov attraction is more effective for large mass ratios $M / m$.

More precisely, for a given partial wave $F_{L M}(\vec{R})=$ $R^{-1} f_{L}(R) Y_{L M}(\hat{R})$ with angular quantum number $L$, this equation can be rewritten in a form similar to equation (4.31),

$$
\left(-\frac{d^{2}}{d R^{2}}+V(R)-\frac{M E}{\hbar^{2}}\right) f_{L}(R)=0,
$$

with the potential

$$
V(R)=\frac{L(L+1)}{R^{2}}+\frac{M}{\hbar^{2}} \epsilon(R)
$$

Identification of this potential at small $R \ll a$ with the form of equation (4.33) gives

$$
\left|s_{0}\right|^{2}=\frac{M}{2 m} \Omega^{2}-L(L+1)-\frac{1}{4}
$$

from which one can calculate the scaling factor $\lambda_{0}=$ $e^{\pi /\left|s_{0}\right|}$ associated with the $1 / R^{2}$ attraction.

If the two heavy particles are distinguishable or identical bosons, one can take $L=0$. The corresponding potential $V(R) \propto \epsilon(R)$ is purely attractive, as shown in figure 6.3. If the two heavy particles are identical fermions, there should be at least one unit of angular momentum between the two heavy particles to respect the antisymmetry of their wave function. There is therefore a competition between the Efimov attraction and the centrifugal repulsion. The resulting potential $V(R)$ is represented in figure 6.4 for different values of the mass ratio. According to equation (6.14), the Efimov attraction wins for large enough mass ratio $M / m$. For $\left|s_{0}\right|=0$, one obtains the critical mass ratio $\kappa_{c} \approx 13.990296$ at which the potential becomes purely attractive, which is very close to the exact result $\kappa_{c} \approx 13.6069657$ presented in section 6.2.2. Above this critical mass ratio, the potential is dominated by the $R^{-2}$ Efimov attraction for $R \ll a$, which leads to the Efimov effect.

The Efimov scaling factor $\lambda_{0}$ obtained from equation (6.14) is shown by dashed curves in figure 6.2 for both the bosonic $L=0$ and fermionic $L=1$ cases, and is in good agreement with the exact results for large mass ratios. The Born-Oppenheimer approximation thus gives a simple account of the Efimov effect for $2+1$ particles. 


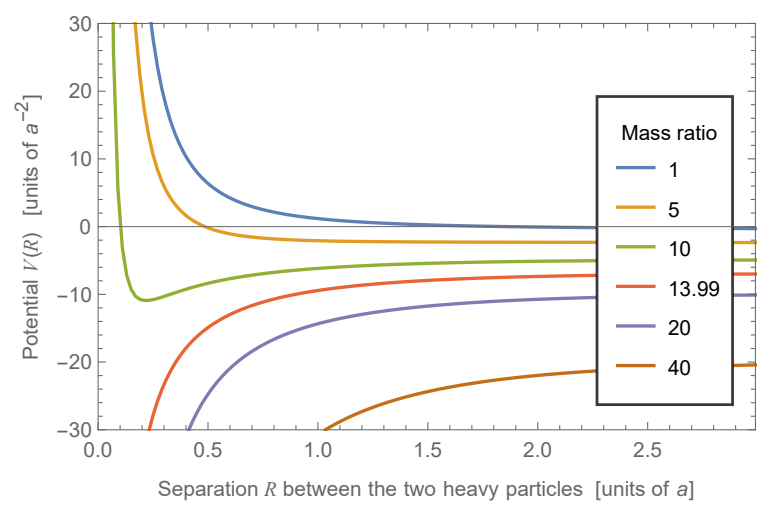

Figure 6.4: Effective potential $V(R)$ in the BornOppenheimer approximation between two identical fermions separated by $R$ and resonantly interacting (with a scattering length $a>0$ ) with a light particle, for various values of their mass ratio. This potential results from the competition between the bonding potential $\epsilon(R)$ and the centrifugal repulsion with one unit of angular momentum - see equation (6.13). At short separations $R \ll a$, the potential exhibits the $\propto R^{-2}$ Efimov attraction for a mass ratio larger than the critical value $\kappa_{c} \approx 13.99$ (corresponding to the red curve), while it is repulsive for smaller mass ratios.

\subsubsection{Kartavtsev-Malykh universal trimers}

As we saw in the preceding sections, a system of two identical fermions resonantly interacting with a light particle can be bound by the Efimov effect when the mass ratio between the fermions and the light particle is larger than a critical value $\kappa_{c}$. Nevertheless, even below the critical mass ratio, it is possible for the system to form universal three-body bound states with the same quantum numbers for $a>0$. This fact was pointed out by Oleg I. Kartavtsev and Anastasia V. Malykh [183]. It can be seen from the BornOppenheimer potential $V(R)$ between the two fermions shown in figure 6.4. For a mass ratio smaller than $\kappa_{c}$, even though the centrifugal repulsion wins over the Efimov attraction, making the potential repulsive at short separation, there can nonetheless be an attractive part at larger distances, thereby creating a potential well. This potential well can be seen in the curve corresponding to a mass ratio of 10 in figure 6.4. The potential well deepens as the mass ratio increases, until it becomes purely attractive at the critical mass ratio. Kartavtsev and Malykh have shown by solving the three-body problem exactly (using the hyperspherical formalism sketched at the beginning of this section) that a ground three-body bound state appears at the critical mass ratio $\kappa_{1} \approx 8.17260$, and an excited one appears at the mass ratio $\kappa_{2} \approx 12.91743$. Since there is no Efimov attraction at short distance for these states, there is no need to introduce a three-body boundary condition, and thus the states are universally determined by the scattering length $a>0$ between a fermion and the light particle. Unlike Efimov trimers, the energy of these trimer simply scales with the universal dimer energy. For this reason, they are called "universal trimers". Similar states appear for higher angular momenta at some critical mass ratios [188, 186], connecting to the non-zero angular-momentum Efimov trimers of section 6.2.3 appearing at larger mass ratios.

The existence of these states have important consequences for inelastic three-body collisions by recombination into dimers [190] and the scattering of dimers of fermions with another fermion [191], even at mass ratios slightly lower than $\kappa_{1}$, as was confirmed experimentally [192]. However these states have not been directly observed yet. It should be noted that the universality of these states is in practice limited to very large scattering lengths [193, 194]. Although there is formally no need to introduce a short-range three-body boundary condition for the universal states, such condition exists physically at a separation of the particles on the order of the interaction range $b$. Even when the scattering length is more than ten times that range, the trimers may be significantly affected by the three-body boundary condition.

In reference [193] the $2+1$ fermions problem was solved with a three-body boundary condition implemented by imposing a cutoff at some momentum $b^{-1}$. For mass ratio $\kappa_{1}<\kappa<\kappa_{2}$, a trimer was found to exist on the positive scattering length side and shows the universal features predicted by Kartavtsev and Malykh when the scattering length is very large $a / b \gg 1$ (see figure 6.5). As the scattering length is tuned away from unitarity, however, the binding energy of the trimer gets smaller than that of the universal trimer, due to the three-body boundary condition. A similar behaviour is found for mass ratios $\kappa_{2}<\kappa<\kappa_{c}$, where two universal trimers appear near unitarity (see the central column of figure 6.5) but gradually turn into what the authors of reference [193] called "crossover trimers", which end up dissociating into a fermion and a fermion-light-particle dimer as the scattering length is varied further. The crossover trimers depend on the three-body parameter set by the three-body bounary condition and smoothly connect the universal trimers to the Efimov trimers appearing at larger mass ratio $\kappa \geq \kappa_{c}$ : as the mass ratio is increased from below with a fixed positive scattering length, the universal trimers become more and more sensitive to the three-body parameter, turning into the crossover trimer states, and then at $\kappa \geq \kappa_{c}$ turn into the ground and first excited states of the Efimov trimers (the second and higher excited Efimov trimers start to appear at $\kappa>\kappa_{c}$ ). This scenario illustrated in figure 6.5 describes how the two universal trimers for $\kappa<\kappa_{c}$ smoothly connect to an infinite series of Efimov states for $\kappa>\kappa_{c}$.

The $2+1$ fermions problem was subsequently studied by Arghavan Safavi-Naini and co-workers in reference [194] with a general three-body boundary condition implemented by setting the logarithmic derivative 


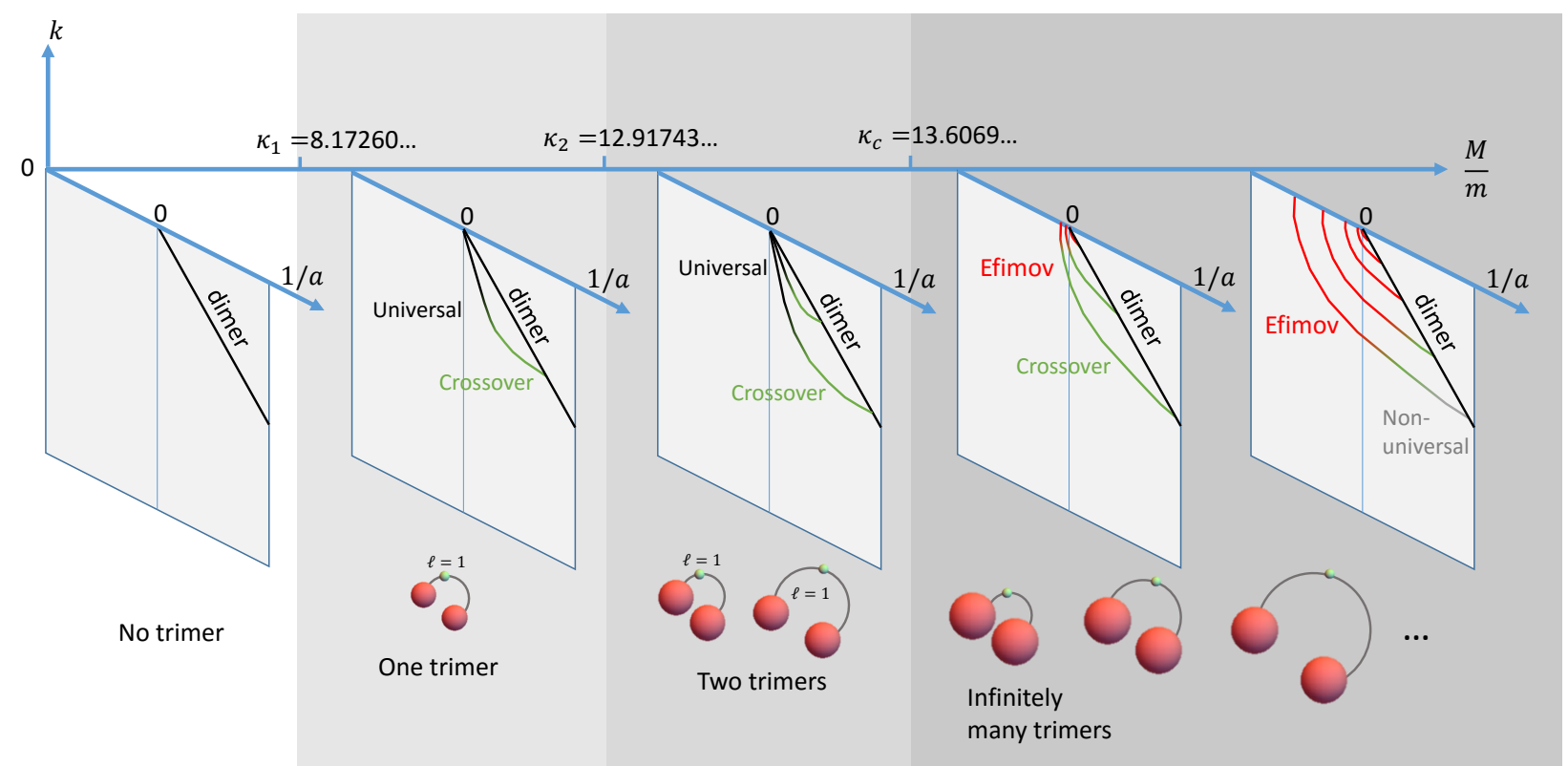

Figure 6.5: Schematic three-body energy spectrum (scaled as a wave number $k$ ) of two identical heavy fermions resonantly interacting with a light particle, as a function of inverse scattering length $1 / a$ between the heavy and light particles, and mass ratio $M / m$ between heavy and light particles. The three-body boundary condition is set by a cutoff in momentum. A trimer appears above the critical mass ratio $\kappa_{1}=8.17260 \ldots$, and a second trimer appears above the critical mass ratio $\kappa_{2}=12.91743 \ldots$. The wave number $k$ of these trimers universally scales with the inverse scattering length $1 / a$ near the unitarity limit $1 / a \rightarrow 0$ (as shown in black) but deviates from this ideal behaviour for larger $1 / a$ (as shown in green). Above the critial mass ratio $\kappa_{c}=13.6069 \ldots$, the Efimov occurs and there is an infinite number of trimers. These trimers exhibit the Efimov discrete scale invariance in the region where the trimer curves are red. The trimer curves shown in green break the discrete scale invariance but are characterised only by the scattering length and a three-body parameter. They are referred to as "crossover trimers" because they dominate at the critical mass ratio $\kappa_{c}$ and make a smooth connection between the universal and Efimov trimers. The trimer curves shown in grey depend on other microscopic details and are referred to as non-universal.

of the hyper-radial wave function at distance on the order of $b$. While this work confirmed that the trimers behave universally when $a / b \gg 1$ and that they tend to become more sensitive to the three-body parameter as the mass ratio is increased towards $\kappa_{c}$, there are some notable differences compared to the above work [193]. In addition to the universal trimers, an additional "nonuniversal" trimer may exist. Here, non-universal means that it strongly depends on the three-body boundary condition in addition to the scattering length $a$. As the value of the three-body boundary condition is varied, the non-universal trimer makes avoided crossings with the universal trimers, and shifts their energies away from the universal predictions. In contrast to the momentum cutoff method of reference [193], the shift can be either positive or negative depending on binding energy of the non-universal state. When it is bound deeper than the universal trimers, it pushes up their energies. On the contrary, it pushes down the energies of the universal trimers when it lies above them.

In the limit where the scattering length $a$ is much larger than $b$, the three-body boundary condition can be implemented as a zero-range boundary condition parameterised by a three-body parameter $\Lambda$ (as in equation (4.35)) for mass ratios larger than $\kappa_{r} \approx 8.619$, because both the regular solution (which vanishes at small hyper-radius) and the irregular solution (which diverges at small hyper-radius) are square-integrable in this case $[195,194,196]$. The precise energy spectrum in this limit for all possible values of $\Lambda|a|$ and all mass ratios $\kappa_{r}<\kappa<\kappa_{c}$ has recently been calculated by Kartavtsev and Malykh [196] for both $a>0$ and $a<0$. In some range of $\Lambda|a|$, the trimers may appear, like Efimov states, at a negative scattering length instead of being restricted to $a>0$. For $\Lambda^{-1} \ll a$, one retrieves the universal results.

To answer which kind of three-body boundary condition should be taken for a given physical system, one needs further knowledge on the microscopic details of the system in question. Indeed, recent works on the microscopic origin of the three-body parameter suggest that pairwise interactions inducing a significant drop of 
probability at short pair separation create an effective repulsive barrier that serves as a three-body boundary condition. This subtle effect is discussed in detail in section 11. Whether it is also relevant here is an open question.

\subsection{Particles with spin}

In many systems occurring in nature, particles have an internal spin that complicates somewhat their description.

\subsubsection{Rotationally invariant systems}

In many cases, the interaction between two particles 1 and 2 is rotationally invariant, and thus does not depend on the spin projection numbers but only on the spin quantum number $s$ for the total spin of the two particles. For example, for particles with spin $1 / 2$, there are two interaction potentials: the singlet potential (for $s=0$ ) and the triplet potential (for $s=1$ ). A general treatment of the resonantly-interacting threebody problem with spin has been given by Bulgac and Efimov [197]. In general, for a given total spin $S$ of the three-body system, one can generalise the Faddeev decomposition of equation (4.16) as follows [4]:

$$
\begin{aligned}
\Psi_{S}(1,2,3)= & \sum_{s} \chi_{s}^{(1)}\left(\vec{r}_{23}, \vec{\rho}_{23,1}\right)|s\rangle_{23} \\
& +\sum_{s} \chi_{s}^{(2)}\left(\vec{r}_{31}, \vec{\rho}_{31,2}\right)|s\rangle_{31} \\
& +\sum_{s} \chi_{s}^{(3)}\left(\vec{r}_{12}, \vec{\rho}_{12,3}\right)|s\rangle_{12}
\end{aligned}
$$

The states $|s\rangle_{i j}$ denote three-spin states of total spin quantum number $S$ and spin quantum number $s$ for the pair $i j$. For a given pair $i j$, these states form a basis of the three-spin space of total spin $S$. One can thus express the states for the other pairs as a linear combination of the states for that particular pair. In section 6.4 .1 , we illustrate this by treating the specific example of the triton.

\subsubsection{Polarised systems}

In some other cases, such as in the presence of a magnetic field that strongly breaks the rotational invariance, the pairwise interaction can be assumed to depend only on the spin projections (magnetic quantum number $m$ ) of the two particles. Then, each spin projection can be regarded as a different kind of particle, and the situation is equivalent to the cases discussed in sections 6.1, 6.2.1, and 6.2.2. For instance, in the experiments discussed in section 6.4 of lithium- 6 atoms in a magnetic field, polarised in three different hyperfine states $|a\rangle,|b\rangle$, and $|c\rangle$, the pairwise interaction just depends on the hyperfine states of the pair [79]. Although the atoms are fermionic, the fully antisym- metrised wave function for three atoms in three different spin states reads:

$$
\begin{aligned}
\Psi(1,2,3)= & \phi(1,2,3)|a, b, c\rangle-\phi(1,3,2)|a, c, b\rangle \\
+ & \phi(2,3,1)|b, c, a\rangle-\phi(2,1,3)|b, a, c\rangle \\
+ & \phi(3,1,2)|c, a, b\rangle-\phi(3,2,1)|c, b, a\rangle
\end{aligned}
$$

and therefore is equivalent to a single wave function $\phi$ describing a system of three distinguishable particles, with identical masses but three different scattering lengths $a_{12}, a_{23}$, and $a_{31}$ - see section 6.1 for the derivation of the Efimov effect in this system.

\subsubsection{Spin-orbit interaction}

Spin-orbit interaction is the coupling between a particle's spin and its motion. It occurs for charged particles such as electrons in atoms and solids, as well as nucleons inside a nucleus. In the recent years, it has also been possible to create artificial spin-orbit interaction for neutral atoms using laser techniques [198]. The influence of the spin-orbit interaction on Efimov physics was addressed by Zhe-Yu Shi, Xiaoling Cui, and Hui Zhai [199, 200]. They considered a system of two heavy fermions resonantly interacting with one light particle, in which the light particle is a spin- $1 / 2$ particle subject to an isotropic spin-orbit interaction of the form $\lambda \vec{p} \cdot \vec{\sigma}$, where $\vec{p}$ is the particle's momentum and $\vec{\sigma}$ its spin. From their calculations, some general conclusions can de drawn.

First of all, the spin-orbit interaction lowers the energy of the heavy-light dimer and heavy-heavy-light trimers. However, the spin-orbit interaction competes with the Efimov attraction and reduces the binding energy of Efimov trimers with respect to the particledimer threshold. Since the spin-orbit interaction only affects large distances, while the Efimov attraction persists at shorter distances, the critical mass ratio $\kappa_{c}$ for the onset of Efimov states, the discrete scale invariance and scaling ratio $\lambda_{0}$ remain unchanged. The discrete scale invariance is however broken below a certain energy scale associated with the coupling strength $\lambda$, making the number of trimers finite, as the excited trimers are pushed into the particle-dimer threshold.

On the other hand, below the critical mass ratio, the spin-orbit interaction favours the appearance of Kartavtsev-Malykh-like universal trimers in a broader range of scattering lengths and mass ratios. In the presence of spin-orbit coupling, these universal trimers not only exist for positive scattering lengths but in a range of negative scattering lengths as well, and the ground-state trimer appears at mass ratio 2.68 (for the state with total angular momentum $J=1 / 2$ ) and 5.92 (for $J=3 / 2$ ).

\subsection{Observations in nuclear physics}

So far, all known multicomponent systems in nuclear physics related to Efimov physics involve two neutrons 
as two of the three particles. Neutrons are favourable for the following reasons:

- First of all, the interaction between two neutrons is resonant, the basic requirement for Efimov physics, since the neutrons can almost form a two-body bound state.

- Second, they carry a spin $1 / 2$, and can therefore be in two distinguishable states, or more precisely they can form a spin singlet state. This particular configuration is free of the centrifugal repulsion that is otherwise present for fermions in a triplet state (such as two identical fermions) and would suppress the Efimov attraction.

- Third, neutrons having no electrical charge, unlike protons, they do not have any Coulomb repulsion between themselves and other particles, that would compete with the Efimov attraction.

\subsubsection{Triton}

The simplest case is the third particle being another nucleon. It cannot be a neutron, since it would have to be in the same spin state as one of the two others, and the resulting Fermi repulsion would suppress the Efimov attraction. As seen in section 6.2.2, the Efimov attraction for two identical fermions and one particle of equal mass is not strong enough to overcome the Fermi repulsion. As a matter of fact, there is no bound state of three neutrons. The third nucleon therefore has to be a proton, and the three-body system corresponds to the triton, the nucleus of tritium ${ }^{3} \mathrm{H}$.

The triton was considered in Vitaly Efimov's original work as a possible candidate for Efimov state. Here, we show how the Efimov attraction explains the binding of the triton and roughly reproduces its binding energy. We first derive the Efimov attraction in the zero-range theory of two neutrons and one proton, and show that is the same as for three identical bosons. Then, we take into account the finite-range corrections, and show how the triton fits in the Efimov spectrum.

Efimov attraction in the triton Systems of nucleons are often described as identical particles with an internal property called isospin, which distinguishes between the neutron state and proton state as different projections of a formal spin $1 / 2$. This is possible because protons and neutrons have nearly the same mass, and nuclear interactions are approximately the same for protons and neutrons, and thus nearly isospinsymmetric. The isospin symmetry has been further confirmed to originate from the symmetries of quantum chromodynamics (QCD) describing nucleons as made of quarks. In the zero-range theory, however, the isospin formalism is not essential, as the nuclear interactions turn out to depend only on spin at this simple level of description. For the sake of readers unfamiliar with isospin, we shall simply describe the triton as two identical fermions (two neutrons) and a distinguishable fermion (proton), and assume that the pairwise nuclear interaction depends only on the total spin quantum number of two nucleons. We also assume the neutron and the proton to have the same mass $m$. For a treatment with isospin, we refer the readers to Refs. [19, 201].

Each nucleon carries a spin $1 / 2$, whose projection on a fixed axis can be either up $|\uparrow\rangle$ or down $|\downarrow\rangle$. The triton is characterised by a total spin $S$ equal to $1 / 2$, and a projection $S_{z}=1 / 2$. Therefore, its wave function can be expressed on the spin basis states,

$$
\begin{aligned}
|0\rangle_{23}= & |\uparrow\rangle_{1} \frac{1}{\sqrt{2}}\left(|\uparrow\rangle_{2}|\downarrow\rangle_{3}-|\downarrow\rangle_{2}|\uparrow\rangle_{3}\right) \\
|1\rangle_{23}= & \frac{1}{\sqrt{3}}|\uparrow\rangle_{1} \frac{1}{\sqrt{2}}\left(|\uparrow\rangle_{2}|\downarrow\rangle_{3}+|\downarrow\rangle_{2}|\uparrow\rangle_{3}\right) \\
& -\sqrt{\frac{2}{3}}|\downarrow\rangle_{1}|\uparrow\rangle_{2}|\uparrow\rangle_{3}
\end{aligned}
$$

which are obtained by standard summation of spins using Clebsh-Gordan coefficients. Here, we assume that particle 1 is the proton, and particles 2 and 3 are the two neutrons. As can be seen from the above expressions, $|0\rangle_{23}$ corresponds to a spin singlet state of the two neutrons, whereas $|1\rangle_{23}$ corresponds to a spin triplet state. The total wave function of the triton is thus:

$$
\Psi(123)=\psi_{S}|0\rangle_{23}+\psi_{A}|1\rangle_{23}
$$

and has to be antisymmetric under the exchange of the two neutrons, particles 2 and 3 . Since the singlet and triplet states are respectively antisymmetric and symmetric under such exchange, as can be checked from equations (6.16-6.17), $\psi_{S}$ and $\psi_{A}$ have to be symmetric and antisymmetric, respectively. The Faddeev decompositions of $\psi_{S}$ and $\psi_{A}$ that preserve their symmetries are:

$\psi_{S}=\chi\left(\vec{r}_{23}, \vec{\rho}_{23,1}\right)+\phi\left(\vec{r}_{12}, \vec{\rho}_{12,3}\right)+\phi\left(\vec{r}_{13}, \vec{\rho}_{13,2}\right)$
$\psi_{A}=\xi\left(\vec{r}_{23}, \vec{\rho}_{23,1}\right)+\zeta\left(\vec{r}_{12}, \vec{\rho}_{12,3}\right)-\zeta\left(\vec{r}_{13}, \vec{\rho}_{13,2}\right)$

where $\chi$ and $\xi$ are respectively even and odd functions of $\vec{r}_{23}$. One can see that the spin singlet configuration allows the spatial configuration $\psi_{S}$ to have the bosonic exchange symmetry when $\phi=\chi$, as in equation (4.16), which in turn allows the Efimov effect to occur.

In the zero-range theory, $\psi_{S}$ and $\psi_{A}$ each satisfy the free Schrödinger equation - see equation (4.15) - with the Bethe-Peierls boundary conditions for the contact of two nucleons, either in the singlet or triplet spin state. In the case of the neutron-neutron interaction, these conditions are readily expressed as:

$$
\begin{aligned}
& \left(\frac{\partial}{\partial r_{23}} r_{23} \psi_{S}\right)_{r_{23} \rightarrow 0}=-\frac{1}{a_{s}}\left(r_{23} \psi_{S}\right)_{r_{23} \rightarrow 0} \\
& \left(\frac{\partial}{\partial r_{23}} r_{23} \psi_{A}\right)_{r_{23} \rightarrow 0}=-\frac{1}{a_{t}}\left(r_{23} \psi_{A}\right)_{r_{23} \rightarrow 0}
\end{aligned}
$$


where $a_{s}$ and $a_{t}$ are the nucleon singlet and triplet $s$-wave scattering lengths. One can then proceed as in section 4.1, retaining only the zero-angular momentum contribution of the Faddeev components of $\psi_{S}$ and $\psi_{A}$, i.e. assuming the form of equation (4.19), and using the hyper-spherical coordinates of equations (4.224.23). This gives a boundary condition analogous to equation (4.26) for the singlet interaction,

$$
\left[\frac{\partial}{\partial \alpha}\left(\chi_{0}(R, \alpha)\right)\right]_{\alpha \rightarrow 0}+\frac{8}{\sqrt{3}} \phi_{0}\left(R, \frac{\pi}{3}\right)=-\frac{R}{a_{s}} \chi_{0}(R, 0)
$$

For the triplet interaction, equation (6.21) gives no constraint on $\psi_{A}$ because it is antisymmetric under the exchange of the two neutrons, i.e. $\psi_{A} \rightarrow 0$ when $r_{23} \rightarrow 0$. This expresses the fact that for zero-range interactions neutrons interact only in the singlet state. For this reason, we can simply set $\xi=0$.

In the case of proton-neutron interactions, we first have to rotate the spin basis to obtain states that are singlet and triplet states of the proton-neutron subsystem (say, particles 1 and 2):

$$
\left(\begin{array}{l}
|0\rangle_{23} \\
|1\rangle_{23}
\end{array}\right)=\left(\begin{array}{cc}
-1 / 2 & -\sqrt{3} / 2 \\
\sqrt{3} / 2 & -1 / 2
\end{array}\right)\left(\begin{array}{l}
|0\rangle_{12} \\
|1\rangle_{12}
\end{array}\right)
$$

This gives

$$
\Psi=\psi_{0}|0\rangle_{12}+\psi_{1}|1\rangle_{12}
$$

with

$$
\psi_{0}=-\frac{1}{2} \psi_{S}+\frac{\sqrt{3}}{2} \psi_{A} \quad ; \quad \psi_{1}=-\frac{\sqrt{3}}{2} \psi_{S}-\frac{1}{2} \psi_{A} .
$$

The boundary conditions are then expressed as:

$$
\begin{aligned}
& \left(\frac{\partial}{\partial r_{12}} r_{12} \psi_{0}\right)_{r_{12} \rightarrow 0}=-\frac{1}{a_{s}}\left(r_{12} \psi_{0}\right)_{r_{12} \rightarrow 0} \\
& \left(\frac{\partial}{\partial r_{12}} r_{12} \psi_{1}\right)_{r_{12} \rightarrow 0}=-\frac{1}{a_{t}}\left(r_{12} \psi_{1}\right)_{r_{12} \rightarrow 0}
\end{aligned}
$$

This gives

$$
\begin{array}{r}
{\left[\frac{\partial}{\partial \alpha}\left(\phi_{0}-\sqrt{3} \zeta_{0}\right)\right]_{\alpha \rightarrow 0}+\frac{4}{\sqrt{3}}\left(\chi_{0}+\phi_{0}+\sqrt{3} \zeta_{0}\right)\left(R, \frac{\pi}{3}\right)} \\
=-\frac{R}{a_{s}}\left(\phi_{0}-\sqrt{3} \zeta_{0}\right)(R, 0)
\end{array}
$$

$$
\begin{array}{r}
{\left[\frac{\partial}{\partial \alpha}\left(\phi_{0}+\frac{\zeta_{0}}{\sqrt{3}}\right)\right]_{\alpha \rightarrow 0}+\frac{4}{\sqrt{3}}\left(\chi_{0}+\phi_{0}-\frac{\zeta_{0}}{\sqrt{3}}\right)\left(R, \frac{\pi}{3}\right)} \\
=-\frac{R}{a_{t}}\left(\phi_{0}+\frac{\zeta_{0}}{\sqrt{3}}\right)(R, 0)
\end{array}
$$

Combining equations (6.22) and (6.23), one obtains a closed condition

$$
\left[\frac{\partial}{\partial \alpha}\left(\tilde{\chi}_{0}(R, \alpha)\right)\right]_{\alpha \rightarrow 0}-\frac{4}{\sqrt{3}} \tilde{\chi}_{0}\left(R, \frac{\pi}{3}\right)=-\frac{R}{a_{s}} \tilde{\chi}_{0}(R, 0)
$$

for the quantity $\tilde{\chi}_{0}=\chi_{0}-\phi_{0}+\sqrt{3} \zeta_{0}$. Unlike equation (4.26) for the bosonic case, this condition itself only yields real eigenvalues $s_{n}$ which do not lead to the Efimov effect. However, unlike the bosonic case, this condition admits the extra solution $\tilde{\chi}_{0}=0$, which does not set the total wave function to zero. In the language of isospin symmetry, this corresponds to considering states with total isospin $T=1 / 2$. Making this choice, the two remaining conditions are:

$$
\begin{array}{r}
{\left[\frac{\partial}{\partial \alpha} \chi_{0}\right]_{\alpha \rightarrow 0}+\frac{8}{\sqrt{3}} \phi_{0}\left(R, \frac{\pi}{3}\right)=-\frac{R}{a_{s}} \chi_{0}(R, 0)} \\
{\left[\frac{\partial}{\partial \alpha}\left(-4 \phi_{0}+\chi_{0}\right)\right]_{\alpha \rightarrow 0}+\frac{4}{\sqrt{3}}\left(-4 \chi_{0}-2 \phi_{0}\right)\left(R, \frac{\pi}{3}\right)} \\
=-\frac{R}{a_{t}}\left(-4 \phi_{0}+\chi_{0}\right)(R, 0) .
\end{array}
$$

Setting $\chi_{0}=f-\varphi$ and $\phi_{0}=f+\frac{1}{2} \varphi$, one obtains

$$
\begin{aligned}
& \frac{\partial f}{\partial \alpha}(R, 0)+\frac{8}{\sqrt{3}} f\left(R, \frac{\pi}{3}\right)=-R\left(\frac{1}{a_{+}} f+\frac{1}{a_{-}} \varphi\right) \\
& \frac{\partial \varphi}{\partial \alpha}(R, 0)-\frac{4}{\sqrt{3}} \varphi\left(R, \frac{\pi}{3}\right)=-R\left(\frac{1}{a_{-}} f+\frac{1}{a_{+}} \varphi\right)
\end{aligned}
$$

where $a_{ \pm}^{-1}=\frac{1}{2}\left(a_{t}^{-1} \pm a_{s}^{-1}\right)$. These equations were first presented in Efimov's original work [1], although their integral version in momentum space had been derived thirteen years earlier by Skorniakov and TerMartirosian [19]. As shown in section 4.1, the advantage of this form is that one can immediately conclude that the system features the Efimov attraction. Indeed, as anticipated from the symmetry of equation (6.18), when both the singlet and triplet scattering lengths are infinite, equation (6.25) reduces to equation (4.26) for bosons at unitarity. Therefore, there occurs an Efimov attraction of the same strength as that of identical bosons, leading to the same scaling ratio $e^{\pi /\left|s_{0}\right|} \approx 22.7$ in the energy spectrum.

Finite-range corrections The above zero-range theory predicts the existence of a two-body bound state of neutron and proton (the deuteron), since $a_{t}>0$, as well as possible Efimov three-body bound states of two neutrons and one proton. Using the deuteron energy $E_{d} \approx 2.224 \mathrm{MeV}$ and neutron-deuteron spin-doublet scattering length $a_{2} \approx 0.65 \mathrm{fm}$ as experimental inputs to determine respectively the values of $a_{t}$ and the threebody parameter in the zero-range theory, one finds one Efimov trimer, and its energy 8.1 MeV [202, 203, 204] is within $5 \%$ that of the triton, $E_{t} \approx 8.48 \mathrm{MeV}$. However, this theory is only qualitative. For instance, using the value $a_{t}=5.4112 \mathrm{fm}$ [205], the predicted binding energy from equation (4.5) for the deuteron is

$$
\frac{\hbar^{2}}{m a_{t}^{2}} \approx 1.416 \mathrm{MeV}
$$



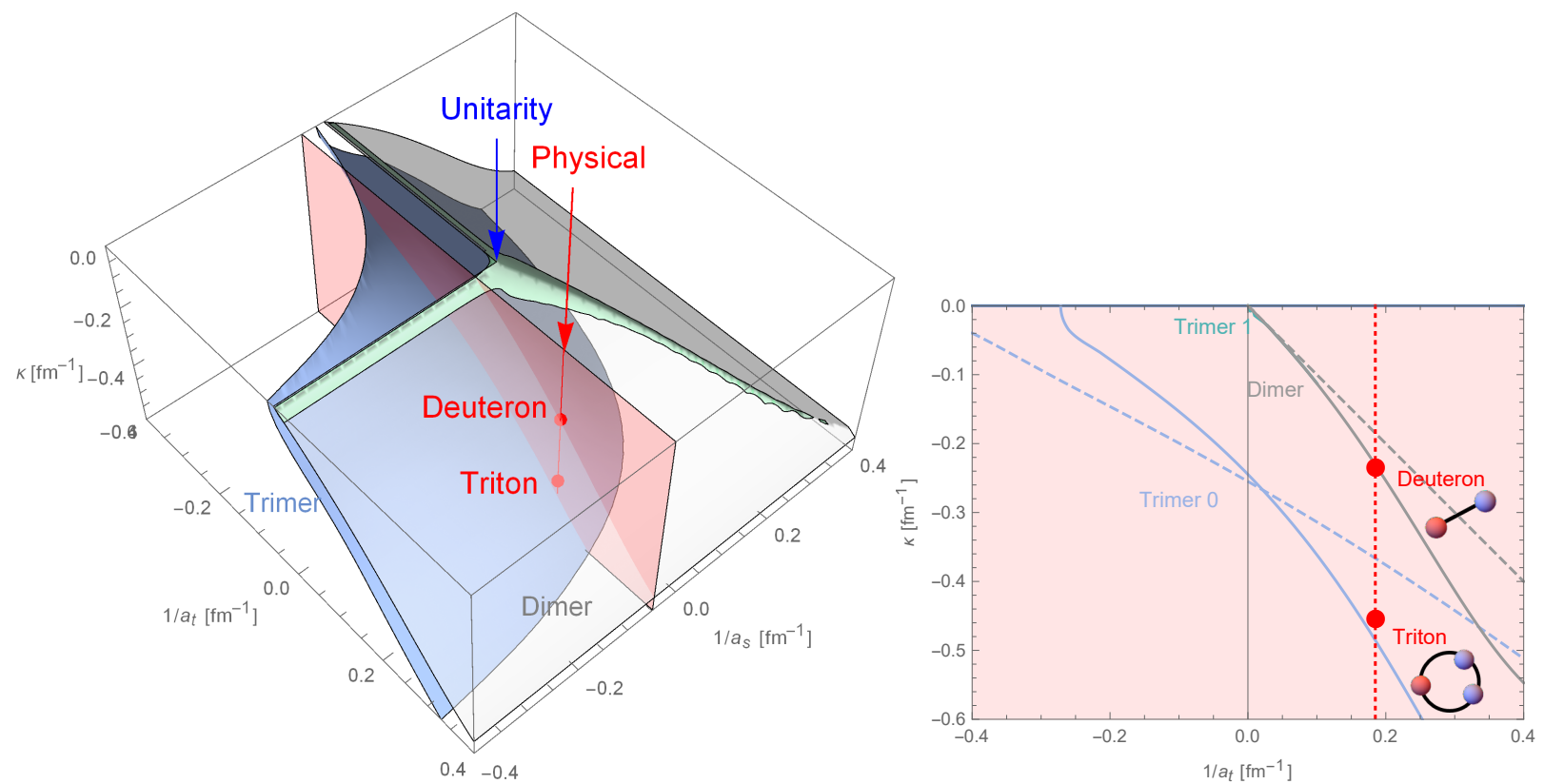

Figure 6.6: (Left) Energy spectrum of two neutrons and one proton, as a function of the inverses of the singlet and triplet scattering lengths $1 / a_{s}$ and $1 / a_{t}$. The energy $E$ (vertical axis) is scaled as a binding momentum $\kappa=-\sqrt{m|E|} / \hbar$. The lowest dimer energy (white transparent surface), the ground-state trimer energy (blue surface), and the first excited trimer energy (green surface) are obtained from a separable model (See Appendix for details). The blue arrow indicates the unitarity point $1 / a_{s}=1 / a_{t}=\kappa=0$ where the Efimov series of trimers accumulate. The physical value of $a_{s}$ is indicated by the red plane, and the physical value of $a_{t}$ is indicated within that plane by the red line. The red dots on that line indicate the experimental values for the energies of the deuteron and the triton.

(Right) Section of left figure in the red plane. The excited trimer is barely visible. The dashed curves show the results for the zero-range theory with a three-body parameter set to match the highly-excited states.

which significantly differs from the experimental value $2.224 \mathrm{MeV}$. This means that non-zero range corrections are important.

The effective range theory is more quantitative. From equation (4.41), with the triplet effective range $r_{e, t}=1.7436 \mathrm{fm}$ [205], one obtains the binding energy in the effective range approximation

$$
\frac{\hbar^{2}}{m}\left(\frac{1-\sqrt{1-2 r_{e, t} / a_{t}}}{r_{e, t}}\right)^{2} \approx 2.223 \mathrm{MeV}
$$

which is remarkably close to the experimental value. Taking the effective range correction to first order only, one finds:

$$
\frac{\hbar^{2}}{m a_{t}^{2}}\left(1+\frac{r_{e, t}}{a_{t}}\right) \approx 1.909 \mathrm{MeV}
$$

which differs by $15 \%$ from the experimental value.

As discussed in section 4.2, the first-order effectiverange correction may also be taken into account in the three-body calculations [71]. Using $a_{2}$ as the threebody input, and $a_{s}=-23.7 \mathrm{fm}[205]$ and $r_{e, s}$ as twobody inputs, V. Efimov and E. G. Tkachenko [203] obtained $8.8 \mathrm{MeV}$ for the energy of the trimer (using $r_{e, t}=1.75 \mathrm{fm}$ and $r_{e, s}=2.67 \mathrm{fm}$ ), while P. .F. Bedaque and co-workers [204] obtained $8.3 \mathrm{MeV}$ (using $r_{e, t}=1.764 \mathrm{fm}$ and $r_{e, s}=2.73 \mathrm{fm}$ ) in the framework of effective field theory. Thus, once the effective-range corrections are taken into account, the predicted trimer energy remains just a few percent away from the triton energy. That is because the main effect of range corrections is to shift both the dimer and trimer energies by a similar amount. These results show that the binding of the triton is consistent with the Efimov scenario.

Explanation of the Phillips line The zero-range theory, with or without finite-range corrections, requires a three-body input to set the three-body energy, such as the neutron-deuteron spin-doublet scattering length $a_{2}$. It therefore implies a correlation between the trimer energy and that scattering length. Such correlation was observed numerically for three-nucleon systems and known as the Phillips line [206]. If one plots the results for the triton energy $E_{t}$ and neutrondeuteron scattering length $a_{2}$ obtained from various nucleon-nucleon potential models, one finds that the points $\left(E_{t}, a_{2}\right)$ tend to form a line. V. Efimov and E. G. Tkachenko [202, 203] pointed out that the zero-range theory gives a natural explanation for this fact. Since different nucleon-nucleon potentials lead to slightly different scattering lenths and three-body parameters, their results sample a small portion of the curve re- 
lating $E_{t}$ to $a_{2}$ in the zero-range theory, thus forming a small line. The fact that they do so shows the relevance of the zero-range picture for these systems.

Triton and deuteron in the Efimov spectrum To visualise how the triton and deuteron fit into the Efimov spectrum, we have represented in figure 6.6 the energy spectrum of two neutrons and one proton as a function of $1 / a_{s}$ and $1 / a_{t}$. To take into account the effect of the effective range, the energy is calculated using a separable interaction model [207] (see Appendix for details), which is parameterised to reproduce the values [205] of the effective ranges $r_{e, t}=1.7436 \mathrm{fm}$ and $r_{e, s}=2.750 \mathrm{fm}$ at the physical values of $a_{t}$ and $a_{s}$. Such a model accurately reproduces the effectiverange approximation of dimer energy given by equation (6.27). Moreover, it determines the trimer energy without requiring to set a three-body parameter from a three-body input. The trimer energy depends on the particular form of the separable interaction [207], but as we shall see in section 11.3 , it is roughly determined by the effective ranges only.

The energy surfaces shown in figure 6.6 are the ground and excited trimers and the singlet and triplet dimers. Near the unitarity point $\left(1 / a_{s}, 1 / a_{t}\right)=(0,0)$, the spectrum is discrete-scale-invariant by simultaneous scaling of $a_{s}$ and $a_{t}$ by a factor of $e^{\pi /\left|s_{0}\right|} \approx 22.7$. The physical values of $a_{s}$ and $a_{t}$ correspond to a vertical line in that figure, where the experimental energies of the deuteron and the triton are indicated. From this figure, one can conclude that the deuteron is the triplet dimer, and the triton connects to the ground state of an Efimov series of trimers which accumulates at $\left(1 / a_{s}, 1 / a_{t}, \kappa\right)=(0,0,0)$.

To reproduce the experimental properties of the deuteron and the triton with a higher accuracy, it is of course necessary to construct more sophisticated models building in the details of nuclear interactions (threebody force in particular), coupling of partial waves, the difference of mass between the proton and the neutron, etc. Nonetheless, the Efimov scenario gives a simple understanding of the deuteron and the triton.

\subsubsection{Two-neutron halo nuclei}

Other candidates for Efimov physics in nuclear systems are halo nuclei $[13,15,16,208]$. Halo nuclei are exotic nuclei discovered from the 1980s, which have an anomalously large mean radius and small binding energy. They usually correspond to neutron-rich or proton-rich nuclei, or excited states of normal nuclei, which means that their lifetime is usually short, on the order of a few up to hundreds of milliseconds. Light halo nuclei are shown in figure 6.7. Numerous experiments and analyses have determined that they can be described as a compact core nucleus surrounded by one or few loosely bound nucleons forming a diffuse halo around the core [209]. Examples of one-neutron ha- los are beryllium-11 and carbon-19. Examples of twoneutron halo nuclei are helium-6, lithium-11, boron-17, boron-19 and carbon-22. Helium- 8 is considered to be a four-neutron halo nucleus.

At first sight, halo nuclei look very similar to the situation described by the zero-range theory of section 4.1. The one-neutron halos can be recognised as the universal dimer supported by a resonant short-range interaction, assuming that the effective interaction between the neutron and the core is resonant in the $s$ wave. It is thus tempting to identify the two-neutron halo nuclei with Efimov states composed of a core and two neutrons, in accordance with the discussion at the beginning of section 6.4. This identification would naturally explain a number of features such as the large extent of halo nuclei and the borromean nature of some of them.

However, there may also be significant differences preventing the identification of halo nuclei with Efimov states. For instance, the two-neutron halo nucleus of helium- 6 is known to feature a $p$-wave resonance between the core and one neutron, in addition to their $s$-wave interaction. As seen in section 5.1, $p$-wave resonances do not lead to the discrete scale invariance of Efimov physics. Therefore, halo nuclei such as helium6 cannot be considered as Efimov states, although they may be described by universal models [210]. Another difficulty is that one would expect to find Efimov states by adding one neutron to the one-neutron halo nuclei ${ }^{11} \mathrm{Be}$ and ${ }^{19} \mathrm{C}$, but ${ }^{12} \mathrm{Be}$ and ${ }^{20} \mathrm{C}$ are not currently recognised as two-neutron halo nuclei. It is usually thought that for these systems, the second neutron forms a Cooper pair with the first neutron, which condenses with the other pairs of the core, making the nucleus more compact. However, it is not excluded that ${ }^{12} \mathrm{Be}$ and ${ }^{20} \mathrm{C}$ may constitute Efimov ground states nonetheless. Indeed, the experimental characterisation of halo nuclei relies on the measurement of anomalously large cross sections and narrow momentum distributions of neutrons. While such features are expected for a ground Efimov state close to the two-body or threebody thresholds where its size becomes very large, the ground Efimov state for interactions near unitarity is relatively compact, with a typical size only marginally larger than the range of interactions. This situation could explain why the nuclei obtained by adding one neutron to a one-neutron halo nucleus are more compact and not recognised as halo states. The assumption that the core remains inert in such compact states is however questionable.

On the other hand, there exist two-neutron halo nuclei, such as ${ }^{11} \mathrm{Li}$ and ${ }^{22} \mathrm{C}$, with no corresponding oneneutron halo nucleus. These nuclei could therefore correspond to borromean Efimov states of a core and two neutrons, for which the core-neutron subsystems are unbound but have a virtual state close to threshold (such as ${ }^{10} \mathrm{Li}$ which is observed as a resonance). Such Efimov states are typically much larger in size than 


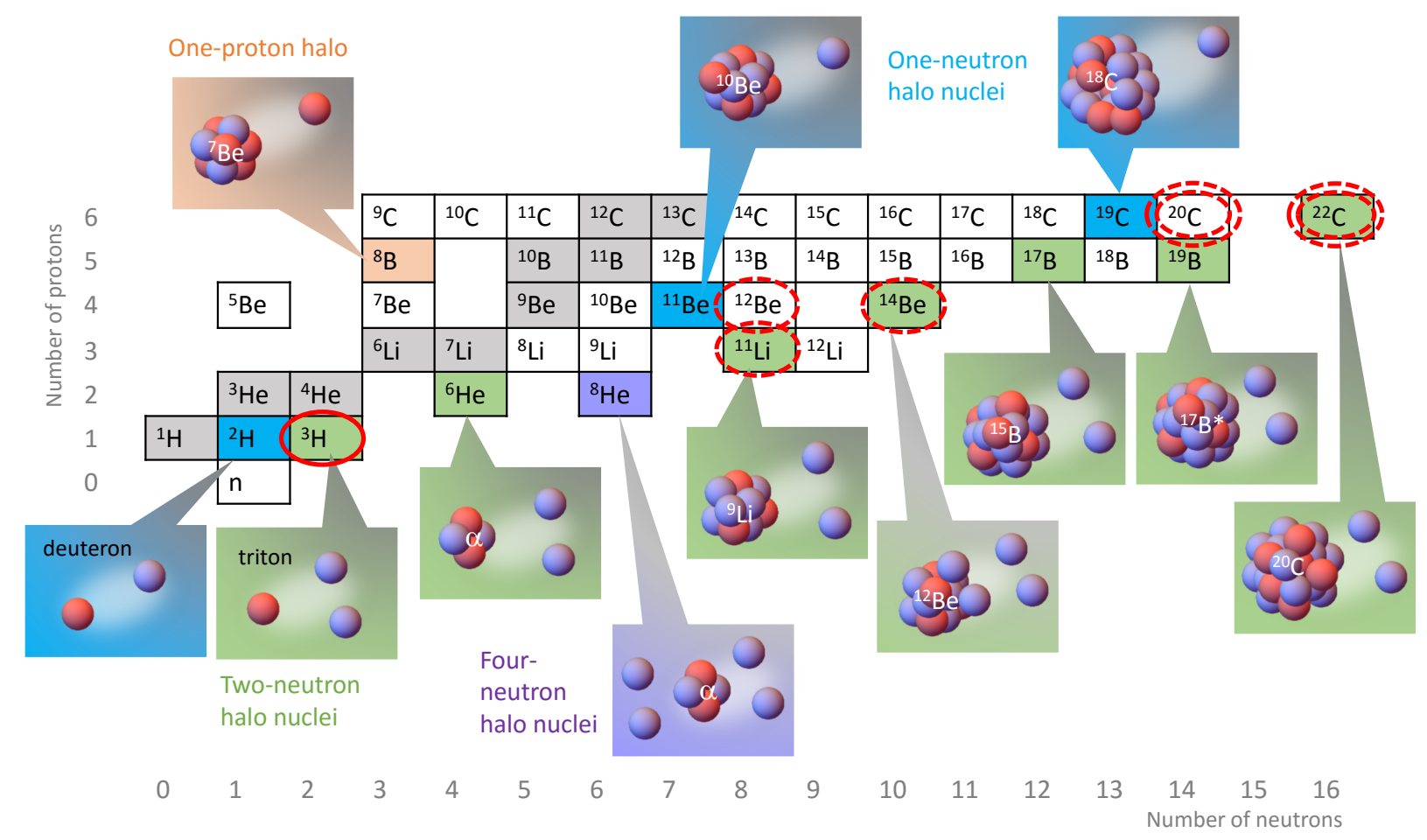

Figure 6.7: Table of nuclides, as a function of the number of protons and number of neutrons, showing stable elements (grey) and observed light halo nuclei: one-proton halo (orange), one-neutron halo (blue), two-neutron halo (green), and four-neutron halo (purple). For each halo nuclei, a schematic representation of their structure is given, where red spheres represent protons and blue spheres represent neutrons. Note that ${ }^{14} \mathrm{Be}$ has been argued to be a four-neutron halo nucleus [13]. Note also that the ${ }^{17} \mathrm{~B}$ core of the two-neutron halo of ${ }^{19} \mathrm{~B}$ does not have the same structure as the halo nucleus of ${ }^{17} \mathrm{~B}$. The red circles indicate possible candidates for ground-state Efimov trimers. The double circle indicates the candidates for excited Efimov trimers. The solid circle denotes a rather established one, while the dashed circles are more conjectural.

the range of interactions, which would explain the halo structure of these nuclei.

In summary, although not all two-neutron halo nuclei are manifestations of Efimov states $\left({ }^{6} \mathrm{He}\right.$ is not), some halo nuclei (such as ${ }^{11} \mathrm{Li}$ and ${ }^{22} \mathrm{C}$ ) are possibly ground-state Efimov trimers in the borromean regime, and other nuclei which are not experimentally regarded as halo nuclei (such as ${ }^{12} \mathrm{Be}$ and ${ }^{20} \mathrm{C}$ ) could be ground-state Efimov trimers close to the unitary limit. For these nuclei, all interactions (core-neutron and neutron-neutron) are assumed to be resonant, and the neutrons can be in a symmetric state (spin singlet). If the core has spin zero, the situation is equivalent to that of two identical bosons and one particle with three resonantly-interacting pairs, as described in section 6.2.1 and illustrated in Fig. 6.2. Since the core is at least nine times heavier than the neutron, the Efimov scaling ratio $\lambda$ when both the neutron-neutron and core-neutron scattering lengths are infinite should be close to the limiting value $\approx 15.74$ for small mass ratios, which is marginally smaller than the scaling ratio for identical bosons $\approx 22.7$. As discussed in section 4.5, the ground state of an Efimov series, though more easily observable, is difficult to characterise as an
Efimov state due to important finite-range corrections. The excited states, on the other hand, conform more clearly to the universal properties of Efimov states, but are less likely to exist and more difficult to observe.

Efimov models of nuclei The first theorists to consider the possibility of Efimov nuclei made of a core plus two neutrons under these assumptions are Dmitri V. Fedorov, Aksel S. Jensen, and Karsten Riisager [24] in 1994. They solved the Faddeev equations for such three-body system, assuming Gaussian potentials for the neutron-neutron and neutron-core interacions, and concluded that ${ }^{14} \mathrm{Be},{ }^{18} \mathrm{C}$ and ${ }^{20} \mathrm{C}$ are possible candidates for Efimov states. The Efimov character of nuclei such as ${ }^{11} \mathrm{Li},{ }^{14} \mathrm{Be}$, and ${ }^{22} \mathrm{C}$ was also investigated using three-body models with separable interactions [211, 212, 213, 214].

In 1997, A. E. A. Amorim, Tobias Frederico, and Lauro Tomio [215] solved the same three-body problem for zero-range interactions, and plotted the region of existence of the $(N+1)$ th Efimov state, which is universally determined by the core-neutron and neutronneutron scattering lengths normalised by the $N$ th Efimov state's energy. Assuming that nuclei such as ${ }^{12} \mathrm{Be}$, 
${ }^{18} \mathrm{C},{ }^{20} \mathrm{C}$ are ground-state Efimov trimers $(N=0)$, and using the measured energies of core-neutron bound states (or virtual states) and neutron-neutron scattering length, the authors could represent the locations of these nuclei in this plot. Only one nucleus, carbon20 , enters the region of existence of the first excited Efimov state $(N=1)$. Carbon-20 lies in fact just at the region boundary, and the large uncertainty on the core-neutron bound state energy (the one-neutron halo nucleus of carbon-19) makes it possible for an excited Efimov trimer state to exist either as a bound state or a virtual state [216, 217].

In 2008, David L. Canham and Hans-Werner Hammer [218] refined the zero-range calculations of Amorim and co-workers in the framework of effective-field theory and estimated the errors due to finite-range corrections. The same authors included the finite-range corrections explicitly in a later work [219]. They reached the same conclusion that among the nuclei ${ }^{11} \mathrm{Li},{ }^{12} \mathrm{Be}$, ${ }^{14} \mathrm{Be},{ }^{18} \mathrm{C}$, and ${ }^{20} \mathrm{C}$, carbon-20 is the only candidate for the existence of an excited Efimov state. As mentioned before, this analysis assumes that the ground state of these nuclei is a ground Efimov state, i.e. the threebody parameter of the zero-range theory is adjusted to reproduce the ground-state energy of these nuclei ${ }^{12}$. Even if the ground state of ${ }^{20} \mathrm{C}$ turns out to be a compact nucleus that has no connection with the Efimov attraction, it could be that an excited state features two neutrons sufficiently far from the core to conform to the structure an Efimov excited state. In this case, however, the ground-state energy of ${ }^{20} \mathrm{C}$ cannot be used to predict the energy of that excited state.

In 2010, carbon-22 was experimentally identified as a two-neutron halo nucleus, exhibiting a root-meansquare matter radius of $5.4 \pm 0.9 \mathrm{fm}$ [220] - recently refined to $3.44 \pm 0.08 \mathrm{fm}$ [221]. Although its two-neutron separation energy has not been measured, the radius value could be used to parameterise three-body models $[222,223]$, constraining the two-neutron separation energy to be smaller than $100 \mathrm{keV}$ (400 keV with the latest radius value [224]). This small two-neutron separation energy imposes the core-neutron scattering length to be very large (corresponding to a virtual state energy well below $1 \mathrm{keV}$ ) in order to allow an excited Efimov state. Experimental data indicates that this is not the case [225], and it is thus likely that carbon-22, like all other borromean two-neutron halo nuclei, does not admit an excited Efimov state.

Comparison with experiments The fitting of a single observable such as the energy or the radius of the nucleus does not of course demonstrate the validity of the three-body models. To confirm the assumption that the ground state of the considered nuclei is an Efimov trimer, the authors of the above-mentioned works have calculated various structural properties from their

\footnotetext{
12 or more precisely, the energy required to separate two neutrons, since the core is considered inert in the three-body model
}

three-body model, in order to compare them with experimental data for these nuclei. In particular, they considered the root mean square radii $r_{n}$ and $r_{c}$ of the neutrons and core from the centre of mass, the root mean square $r_{n n}$ and $r_{n c}$ of the neutron-neutron and neutron-core distances, as well as the opening angle $\theta_{n n}$ between the two neutrons [226, 219]. For ${ }^{11} \mathrm{Li}$, the three-body models (with and without range corrections, as they turn out to be small) give $r_{n} \approx 6.5 \mathrm{fm}$, in fair agreement with the experimental values $r_{n}=$ $6.6 \pm 1.5 \mathrm{fm}$ and $r_{n}=6.1 \mathrm{fm}$ reported in Refs. [227, 228].

These results show that the basic properties of the candidate halo nuclei look indeed consistent with Efimov states. The question whether these nuclei can be considered as Efimov states will be answered more clearly in the coming years as more experimental data becomes available, and more comparison between three-body models and more microscopic models like shell models are developed.

\subsection{Observations with atoms}

\subsubsection{Two-component trimers}

Potassium-Rubidium mixtures Efimov states of a mass-imbalanced system have been observed as atomdimer resonances in ultra-cold mixtures of potassium and rubidium atoms [229, 230, 231, 232], in which the external magnetic field is tuned close to a Feshbach resonance between the potassium and rubidium atoms. If both potassium and rubidium atoms are bosonic, as is the case for ${ }^{39} \mathrm{~K},{ }^{41} \mathrm{~K}$, and ${ }^{87} \mathrm{Rb}$ atoms, two kinds of Efimov trimers are possible: KRbRb and KKRb, corresponding respectively to two heavy bosons plus one light particle, and two light bosons plus one heavy particle. Therefore, the ground-state trimers of these two Efimov series should appear at the three-body threshold at some negative scattering length $a_{-}^{K R b R b}$ and $a_{-}^{K K R b}$. As the inverse of the potassium-rubidium scattering length is continuously varied from negative to positive values, these trimers may break up into an atom and a dimer at some positive scattering lengths $a_{*}^{K R b R b}$ and $a_{*}^{K K R b}$. This implies that atom losses in the mixture due to recombination into deeper states are enhanced when the scattering length is tuned around one of these scattering lengths, i.e. one expects to observe peaks in three-body recombination loss rate at $a_{-}^{K R b R b}$ and $a_{-}^{K K R b}$ for the atomic mixture, and peaks in the atom-dimer relaxation loss rate at $a_{*}^{K R b R b}$ $\left(a_{*}^{K K R b}\right)$ for a mixture of rubidium (potassium) atoms and $\mathrm{KRb}$ dimers.

A three-body calculation using potentials with a van der Waals tail [233] has shown that $\left|a_{-}^{K R b R b}\right|>$ $3 \times 10^{4} a_{0}$ and $\left|a_{-}^{K K R b}\right|>1 \times 10^{6} a_{0}$ (where $a_{0}$ is the Bohr radius). It is currently not possible to observe the three-body loss peaks associated with these negative scattering lengths ${ }^{13}$, since it is experimentally difficult

\footnotetext{
${ }^{13}$ In 2009, the group of Massimo Inguscio and Francesco Minardi in Florence [234] claimed to observe the first signatures
} 
to tune the magnetic field to such large values of the scattering length and reach a temperature that is low enough to resolve the peaks [236]. On the other hand, the positive scattering lengths $a_{*}^{K R b R b}$ and $a_{*}^{K K R b}$ take experimentally accessible values.

In 2013, the group of Deborah Jin at JILA measured the three-body loss rate and the atom-dimer relaxation rate in a mixture of potassium-40 and rubidium- 87 atoms. Note that potassium-40 is fermionic, so that the Efimov effect only occurs for KRbRb in this system. While they could not find any evidence of three-body loss peaks for $-3000 a_{0}<a<-200 a_{0}$ [229, 235, 230], they observed a peak in the atom $\left({ }^{87} \mathrm{Rb}\right)$ - dimer $\left({ }^{40} \mathrm{~K}^{87} \mathrm{Rb}\right)$ relaxation rate at the positive scattering length $a_{*}=230(30) a_{0}[229,230]$. More recently, the group of Shin Inouye at the University of Tokyo has also found a peak in the atom-dimer relaxation rate in a ${ }^{41} \mathrm{~K}-{ }^{87} \mathrm{Rb}$ mixture at a similar position $a_{*}=348(49) a_{0}[231,232]$. This agreement can be naturally explained if we interpret these peaks as induced by the Efimov states: since the atoms in both experiments are of the same atomic species with nearly the same mass, and the interatomic interactions should have almost the same van der Waals tail, one naturally expects on the basis of van der Waals universality (see section 11.2 and reference [233]) that both experiments would yield essentially the same results for the KRbRb Efimov physics. Indeed, both peak positions are found to be consistent with $a_{*}^{K R b R b}$ obtained by a multi-channel three-body calculation using a van der Waals type of potential [233, 232]. The Efimov states of RbKK, corresponding to the "Efimov-unfavoured" case of one heavy and two light particles, have yet to be observed.

Lithium-caesium mixtures In 2014, in two independent experiments, one led by Cheng Chin at the University of Chicago [41] and the other led by Matthias Weidemüller at the University of Heidelberg [42], experimentalists investigated mixtures of caesium-133 and lithium-6, taking advantage of the large mass ratio to more easily observe Efimov states since the scaling ratio is only 4.88 (compared to 22.7 for bosons of equal mass). Both groups were able to observe three peaks in the atom losses, corresponding to three Efimov states, when the intensity of the external magnetic

of mass-imbalanced Efimov trimers in a mixture of potassium41 and rubidium- 87 atoms. The group found two peaks in the atom loss measurement at the scattering lengths -246(14) $a_{0}$ and $-22\left(\begin{array}{c}+4 \\ -6\end{array}\right) \times 10^{3} a_{0}$ which they assigned to $a_{-}^{K R b R b}$ and $a_{-}^{K K R b}$, respectively. These values, however, turned out to be too small in magnitude compared to the theoretical values. Furthermore, no three-body loss peak has been observed in any potassium $\left({ }^{39} \mathrm{~K},{ }^{40} \mathrm{~K},{ }^{41} \mathrm{~K}\right)$ rubidium-87 mixture by any of the subsequent experiments in the groups of Deborah Jin at JILA [229, 235, 230], Shin Inouye at the University of Tokyo [231, 232], and Jan Arlt at Aarhus University [236]. The work of reference [236] actually reported a three-body loss peak, but attributed it to a $p$-wave two-body resonance (as predicted by theory) rather than an Efimov resonance. field is tuned near a Feshbach resonance at $B=842.75$ $\mathrm{G}$, although the authors of reference [42] could not confirm the validity of the third peak after a careful analysis of the three-body loss rate. The measured ratio of scattering lengths between the first and second peak was found to be $5.1(2)$ in reference [41] and $5.07(6)(13)(2)$ in reference [42], close to the theoretical value 4.88 . The ratio for the second and third peaks in reference [41] was found to be $4.8(7)$. This is so far the most relevant experimental demonstration of the Efimov effect in its strictest sense (definition 1 of section 4.5), which is the geometric accumulation of trimer states below the three-body threshold.

Lithium-Rubidum mixtures In 2015, the group of C. Zimmermann in Tübingen [237] reported the signature of a heavy-heavy-light ground-state Efimov trimer in an ultra-cold mixture of lithium-7 and rubidium- 87 . The loss peak associated with that trimer was found at $a_{-}=-1870(121) a_{0}$, which is consistent with the value expected from van der Waals models, providing further experimental support for the van der Waals universality of the three-body parameter discussed in section 11.2.

\subsubsection{Three-component trimers}

Three experimental groups working on ultra-cold lithium-6 have independently evidenced the presence of Efimov states made of three distinguishable atoms near broad Feshbach resonances: the group of Selim Jochim in Heidelberg [238, 239, 240], the group of Kenneth M. O'Hara at the Pennsylvania State University [241, 242], and the group of Takashi Mukaiyama at the University of Tokyo $[75,78]$. In all experiments, the atoms were prepared in three different hyperfine sublevels, making it possible for three distinguishable particles to form Efimov trimers, as detailed in section 6.1. This also makes the system more unstable by three-body recombination, since nothing prevents three distinguishable particles from approaching each other, unlike the twocomponent Fermi systems where two of three particles are in the same state and therefore undergo Fermi exclusion that limits the three-recombination processes. The experimentalists thus found the first indications of Efimov physics in the strong variation of the threebody losses with external magnetic field. As the intensity of the magnetic field is varied, the scattering lengths between each pair of states is strongly altered, due to the presence of a broad Feshbach resonance for each scattering length. These broad resonances overlap in the range $600-1200$ gauss (region 1), where all three scattering lengths are much larger than the range of atomic interactions, but also make the three scattering lengths relatively large in the range $100-500$ gauss (region 2). Efimov physics is therefore possible in these two regions. If the three scattering lengths diverged at the same intensity of the magnetic field, one could expect an infinity of Efimov trimers in principle, as in the 
case of identical bosons. However, since the scattering lengths diverge at different intensities of the magnetic field, only a finite number of trimers is expected. As it turns out, the scattering lengths in region 1 are large enough to support two Efimov trimers, while region 2 exhibits only a ground-state Efimov trimer.

Observations through three-body losses The experimentalists first reported the observation of a plateau of strong three-body losses in region 2 (larger than the background losses by three orders of magntitude), delimited by a loss peak at around 130 gauss and a much softer peak around 500 gauss. They suggested that Efimov physics could be behind these strong losses. Using the zero-range theory and fitting the three-body parameter to the experimental data, theorists could indeed reproduce qualitatively the observed plateau $[243,244]$ and attributed it to the presence of a single Efimov trimer appearing from the three-body scattering threshold at around 130 gauss and dissociating back into that threshold at 500 gauss [244]. The fitted three-body parameter also made it possible to predict a loss peak in region 1, at around 1160 gauss [243]. Such a peak was indeed observed very soon afterwards at lower temperatures by the group of K. O'Hara, but it was located at a smaller intensity of 900 gauss. The experimentalists identified this peak with the appearance of an excited Efimov trimer, and showed that its location, along with those of the peaks previously observed in region 2, were consistent with roughly the same three-body parameter. This was the first experimental report of an excited Efimov trimer.

Observations through atom-dimer losses Subsequent experiments in Heidelberg and Tokyo [239, 240, 75, 78] confirmed this interpretation by first preparing a gas of dimers of atoms in two different hyperfine states, by sweeping the magnetic field near one of the Feshbach resonances. These large dimers could then relax into deeper states by inelastic collisions with the remaining atoms in the third hyperfine state. By measuring the relaxation rate as a function of the external magnetic fields, the experimentalists found two loss peaks around 600 gauss and 680 gauss. The zero-range theory [245, 79] showed that, qualitatively, these peaks correspond to a ground and excited Efimov trimers dissociating in the atom-dimer scattering threshold. This confirmed the existence of the excited trimer previously observed at the three-body threshold.

Bound-state spectroscopy Finally, both groups in Heidelberg and Tokyo were able to perform a radiofrequency spectroscopy of the excited trimer below the atom-dimer threshold [240, 78]. This was the first bound-state spectroscopy of an Efimov trimer, i.e. the measurement of its binding energy below a scattering threshold rather than its effects at the scattering threshold.
Although the zero-range theory could unambigously interpret the features observed in lithium-6, it could reproduce them only semi-quantitatively. To reproduce all features quantitatively the three-body parameter has to vary with energy $[75,78,79]$ by about $10 \%$, and the inleasticity parameter $\eta$ describing recombination to deep dimers (see section 10.3) has to be magneticfield dependent [246] or energy-dependent [247]. To avoid these extra fitting assumptions, the work of [79] incorporated two-body range corrections and the presence of deeper dimers via a two-channel model with Gaussian separable potentials. While the overall agreement with experiment is good, the model could not reproduce the precise locations of all features with a single set of parameters. Bo Huang and co-workers [248] recently used an updated two-body model of the Feshbach resonances in lithium-6 [249] to extract more accurately the three-body parameter from the data, using a three-body zero-range model. The three-body parameters obtained from regions 1 and 2 still differ by $5 \%$, and deviate by $20 \%$ from the van der Waals universal value observed in other species (see section 11.2). It would be worthwhile to revisit this problem with threebody models that properly incorporate van der Waals physics and the more accurate description of the Feshbach resonances of reference [249]. 


\section{Part III}

\section{Dimensionality}

The dimensionality of space is crucial for the Efimov effect to occur. For three identical bosons in $d$ dimensions, Esben Nielsen and co-workers [184] have shown that the Efimov effect can occur only when

$$
2.3<d<3.8 \text {. }
$$

As a result, for integral dimensions, only $d=3$ leads to the Efimov effect. In principle, the Efimov effect may also happen for fractional dimensions in the allowed range, although this has not been demonstrated explicitly yet.

\section{$7 \quad$ Situation in 1D and 2D}

According to Nielsen's dimension criterion, there is no Efimov physics in one and two dimensions [250, 251]. Still, one- and two-dimensional three-body systems exhibit universal states when their interaction is tuned near the appearance of two-body bound states. However, because of the absence of Efimov attraction, no three-body parameter needs introducing, and these universal states depend only the scattering length, as the universal two-body states. As a result, in the universal region, the energies of three-body and twobody states are proportional to each other, since they both scale with the scattering length, and there are no Borromean states. Moreover, because of the absence of long-range Efimov attraction, the number of threebody bound states is finite. This is consistent with the theorem by S. A. Vugal'ter and G. M. Zhislin [252] stating that few-body systems with finite-range interactions in one or two dimensions can only support a finite number of bound states.

Even though Efimov physics does not occur, we briefly review the situation for these dimensions to contrast them with the Efimov regime, and understand the connection with Efimov physics in confined geometries.

\subsection{One dimension}

In one dimension, for a short-range pairwise interaction of range $b$, the even-parity two-body wave function has the form

$$
\psi(x) \propto|x|-a_{1 \mathrm{D}}
$$

for $b \ll|x| \ll k^{-1}$, where $a_{1 \mathrm{D}}$ is the one-dimensional scattering length and $k$ is the relative wave number. The zero-range theory reproducing equation (7.1) can be obtained by setting the following Bethe-Peierls boundary condition at $x=0$,

$$
-\frac{1}{\psi} \frac{d \psi}{d x} \underset{x \rightarrow 0}{\longrightarrow} \frac{1}{a_{1 \mathrm{D}}}
$$

or by replacing the interaction by a contact potential $\lambda \delta(x)$ of strength $\lambda=-\frac{\hbar^{2}}{\mu a_{1 \mathrm{D}}}$ where and $\mu$ is the twoparticle reduce mass. Unlike in $3 \mathrm{D}$, no renormalisation or regularisation of the delta function is needed here.

In the one-dimensional zero-range theory, there is one two-body bound state of energy

$$
E_{2}=-\frac{\hbar^{2}}{2 \mu a_{1 \mathrm{D}}^{2}}
$$

for positive $a_{1 \mathrm{D}}$ (attractive interactions) and no bound state for negative $a_{1 \mathrm{D}}$ (repulsive interactions).

\subsubsection{Identical bosons}

For identical bosons in one dimensions, the problem admits analytical solutions [253, 254]. For $a_{1 D}>0$, a system of $N$ particles admits exactly one $N$-body bound state of energy $E_{N}^{(0)}=\frac{N\left(N^{2}-1\right)}{6} E_{2}$, therefore the three-body bound state energy is

$$
E_{3}^{(0)}=4 E_{2} .
$$

In fact, there is also a virtual three-body bound state at zero energy $\left(E_{3}^{(1)}=0\right)$ [255], which can be seen by the fact that the particle-dimer scattering length is infinite. It can also be seen by considering particles with different masses, which can exhibit more than one bound state, and see that in the limit of equal masses, the second bound state vanishes at the threebody threshold. In this sense, the case of identical particles is a critical point for the appearance of the second three-body bound state.

\subsubsection{2 particles +1 particle}

The more general situation of two identical bosonic particles $A$ of mass $M$ and one extra particle $X$ of mass $m$ was extensively studied by Oleg Kartavtsev and collaborators [255]. If the coupling strength $\lambda_{A X}$ between two different particles is positive (repulsive interaction) then there is no three-body bound state, irrespective of the coupling $\lambda_{A A}$ between the identical particles. For negative $\lambda_{A X}$ (attractive interaction between different particles), the results depend on the mass ratio $M / m$ and the interaction strength $\lambda_{A A}$ between the two identical particles.

Effect of the mass ratio For mass ratios $M / m \leq 1$, there is at most one three-body bound state. Larger mass ratios favor the existence of an increasingly larger number of three-body bound states.

Effect of the interaction between identical particles When the identical particle interaction is more attractive than the attraction between different particles $\left(\lambda_{A A} \leq \lambda_{A X}\right)$, there is a strongly bound AA dimer, and only one trimer with lower energy, for any mass ratio. For similar attractions $\lambda_{A A}=\lambda_{A X}$ and equal 
masses $M=m$, we retrieve the case of three identical particles. The trimer energy is given by equation (7.4), where $E_{2}=E_{A X}=E_{A A}$. When $\lambda_{A A}$ is reduced to zero (non-interacting identical particles), the trimer energy is reduced to

$$
E_{A A X}^{(0)} \simeq 2.087719 E_{A X}
$$

As one would expect, repulsion between identical particles $\left(\lambda_{A A}>0\right)$ tends to further reduce the trimer energy and the number of trimers. In the limit of strong repulsion $\left(\lambda_{A A} \rightarrow \infty\right)$, there is no trimer for mass ratio $M / m \leq 1$. Interestingly, this situation also describes the case of two non-interacting identical fermions and one extra particle, due to the one-toone correspondence in one dimension between strongly interacting bosons and non-interacting fermions established by Marvin D. Girardeau [256].

Five-body Efimov effect in one-dimension So far our consideration has been restricted to two-body shortrange interactions. It is worthwhile to note that lowdimensional systems with many-body interactions, although unlikely to be realised, may in fact exhibit a many-body Efimov effect. In reference [257], Yusuke Nishida and Dam T. Son found that five identical bosons in one dimension resonantly interacting through a four-body short-range interaction, but without threeand two-body interactions, exhibit a five-body Efimov effect with scaling factor 12.4. The effect survives, although weakened, for four-component bosons, but disappears for fermionic particles.

\subsection{Two dimensions}

In two dimensions, the $s$-wave scattering length $a_{2 \mathrm{D}}$ is always positive. When it is much larger than the range of the interaction, the interaction can be described by the following contact condition on the two-body wave function $\psi(r)$ :

$$
\psi(r) \propto \ln \left(\frac{r}{a_{2 \mathrm{D}}}\right)+O(r),
$$

which plays the role of the Bethe-Peierls condition (4.7) for two dimensions. Such zero-range model supports exactly one two-body bound state of energy $E_{2}$ given by

$$
E_{2}=-4 e^{-2 \gamma} \frac{\hbar^{2}}{2 \mu a_{2 \mathrm{D}}^{2}} .
$$

where $\gamma$ is Euler's constant and $\mu$ is the reduced mass between the two particles. Thus, unlike in $3 \mathrm{D}$ and $1 \mathrm{D}$, there always is a two-body bound state in two dimensions.

\subsubsection{Identical bosons}

For identical bosons in two dimensions with zero-range interactions, one finds two three-body bound states, with energies [250, 184, 258, 259]:

$$
\begin{aligned}
& E_{3}^{(0)}=16.522688(1) E_{2}, \\
& E_{3}^{(1)}=1.2704091(1) E_{2} .
\end{aligned}
$$

The four-body spectrum has also been calculated [260], and it is found that there are two four-body bound states, with energies:

$$
\begin{aligned}
& E_{4}^{(0)}=197.3(1) E_{2}, \\
& E_{4}^{(1)}=25.5(1) E_{2} .
\end{aligned}
$$

\subsubsection{2 particles +1 particle}

The case of two non-interacting identical particles $A$ of mass $M$, each interacting resonantly with one extra particle $X$ of mass $m$ was studied by Ludovic Pricoupenko and Paolo Pedri [261], as well as Filipe F. Belloti and co-workers [262]. The interaction between particles $A$ and $X$ is described by the scattering length $a_{2 \mathrm{D}}$, through the boundary condition (7.6). Three-body bound states are characterised by a principal quantum number $n$ corresponding to excitations of the hyper-radial motion, and an internal angular momentum with a projection quantum number $\ell$.

When the two identical particles are bosons, the internal angular momentum has an even projection quantum number $\ell=0,2,4, \ldots$ etc. The ground-state trimer exists for any mass ratio and has the quantum numbers $n=0$ and $\ell=0$. In the limit $M / m \rightarrow 0$, its energy is given by

$$
E_{A A X} \simeq 2 E_{A X}
$$

and for $M=m$, it is given by

$$
E_{A A X} \simeq 2.36 E_{A X}
$$

Excited trimer states appear as the mass ratio is further increased, and their number presumably grows indefinitely. The first trimers appear at the following mass ratios: $M / m=1.770(n=1, \ell=0)$, $M / m=8.341(n=1, \ell=0), M / m=12.68(n=$ $0, \ell=2), M / m=18.27(n=3, \ell=0), M / m=23.76$ $(n=1, \ell=2)$.

When the two identical particles are fermions, the internal angular momentum has an odd projection quantum number $\ell=1,3,5, \ldots$ etc. For a mass ratio $M / m$ smaller than $3.340^{14}$, there is no three-body bound state. For larger mass ratios, there is a ground-state trimer with quantum numbers $n=0$ and $\ell=1$. As in the bosonic case, the trimer energy and the number of trimer states increase as the mass ratio is increased. Excited trimer states appear at the following mass ratios: $M / m=10.41(n=1, \ell=1), M / m=20.85(n=$ $2, \ell=1), M / m=26.89(n=0, \ell=3), M / m=34.59$ $(n=3, \ell=1), M / m=41.98(n=1, \ell=3)$.

\footnotetext{
${ }^{14}$ The main text of reference [261] reports the value 3.33 , but the value 3.340 given in Table I is more accurate.
} 

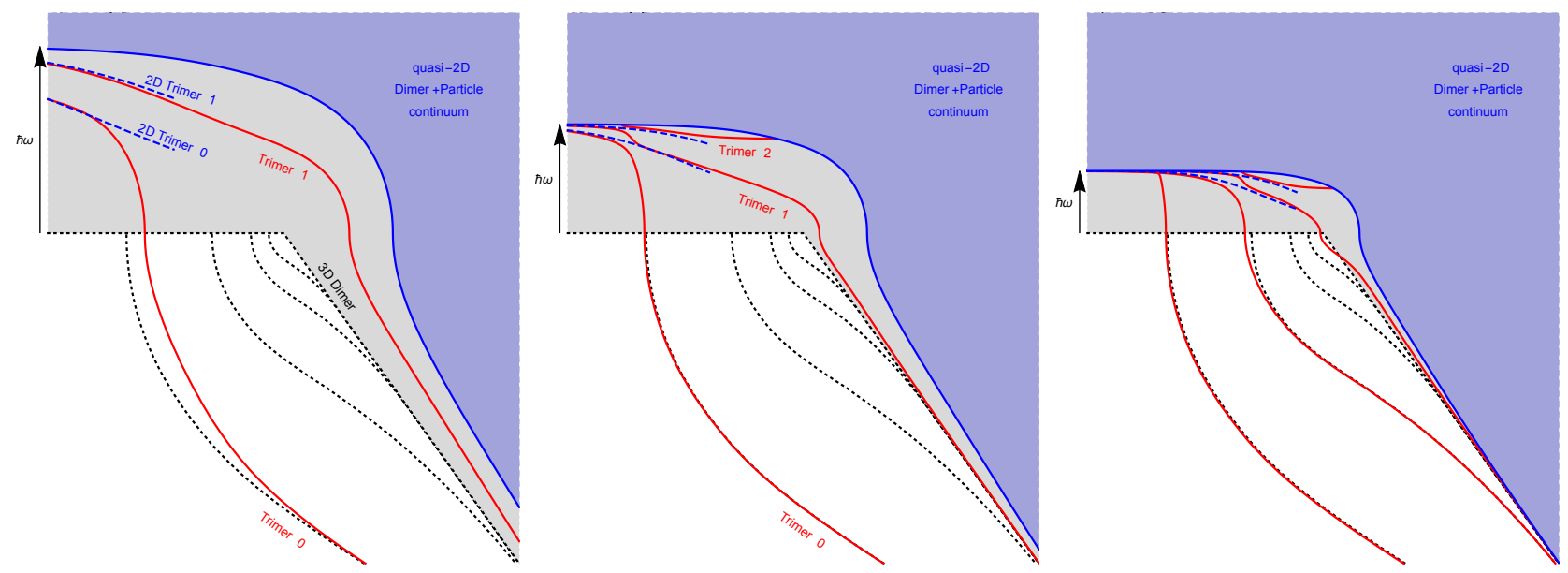

Figure 7.1: Schematic three-body energy spectrum as a function of inverse scattering length, under harmonic confinement in one direction. The three-body bound-state energies are represented by red curves, and the dimer-particle continuum is represented by the dark shaded area. The threshold of this continuum is shifted by $\hbar \omega$ with respected to the free-space three-body threshold. The free-space three-body threshold, along with the free-space dimer and the free-space Efimov trimers are represented in dotted lines for reference - compare with figure 4.2. The left panel corresponds to strong confinement $l \sim a$. In this case there are only two trimers that connect smoothly to the two trimers of the quasi-2D limit (shown in dashed blue curves) for small binding energies. For gradually weaker confinement (middle and right panels), new trimers appear from below the continuum at around $a \sim a_{-}$, exhibiting an avoided crossing structure between free-space states and quasi-2D states. In the limit of weak confinement, one retrieves the free-space spectrum with many Efimov trimers.

\section{Effects of confinement}

In ultra-cold atom experiments, it is possible to create lower-dimensional systems by confining the atoms in a narrow plane or tube by means of laser light. The technique is called optical lattice [263] and consists in shining counter-propagating laser beams onto the atomic cloud. The interference of the beams results in a sinusoidal pattern of light, which creates a one-body potential for the atoms proportional to the light intensity through the Stark effect. Atoms are thus attracted to the nodes of the interference pattern, and repelled by the maxima for a blue-detuned light. With sufficiently strong lasers and appropriate layout of the beams, it is thus possible to confine the atoms in quasi-2D or quasi$1 \mathrm{D}$ geometries. This also gives the interesting possibility of going continuously from a three-dimensional to a lower-dimensional systems. From a theoretical point of view, since the Efimov effect occurs only in three dimensions, it is natural to wonder how the infinity of Efimov states continously transform into a finite number of trimer states at lower dimensions. This problem is reminiscent of the crossover that happens in systems with two heavy fermions and one light particle discussed in section 6.2.5.

\subsection{From 3D to quasi-2D}

In reference [264], Jesper Levinsen, Pietro Massignan, and Meera Parish have looked theoretically into the case of confining Efimov trimers in one direction, thus going from 3D to quasi-2D. They modelled the inter- action by a three-dimensional zero-range interaction of scattering length $a$, and the confinement by a harmonic trap of trapping frequency $\omega$. A harmonic trap is a good approximation of a strongly confining optical lattice when the atoms reside at the bottom of a well in the lattice. It also offers the theoretical advantage of decoupling the centre of mass from the relative motion. Jesper Levinsen and co-workers obtained a version of the Skorniakov and Ter-Martirosian equation for this problem, which they solved numerically.

The two-body spectrum of such a system had already been investigated by Dmitry Petrov and Georgy Shlyapnikov [265] in 2000. The 3D two-body bound state for positive scattering length $a$ is unchanged as long as its extent, given by $a$, is smaller than the confinement length $l=\sqrt{\hbar / m \omega}$. However, when $a \gtrsim l$, the dimer acquires a two-dimensional character. Unlike the free space case, it continues to exist even for negative scattering length $a$, where it becomes a low-energy quasi-2D dimer, whose energy is given by equation (7.7) plus the zero-point energy $\frac{1}{2} \hbar \omega$, which constitutes the energy of the two-body threshold for particles whose motion along the confinement direction is in the ground-state of the harmonic trap. Here, the two-dimensional scattering length is given by:

$$
a_{2 \mathrm{D}}=2 \sqrt{\frac{\pi}{B}} e^{-\gamma} l \exp \left(-\sqrt{\pi / 2} \frac{l}{a}\right)
$$

where $B \approx 0.915$ is a numerical constant and $\gamma$ is Euler's constant. Thus, unlike the free space case, a dimer exists for any value of the 3D scattering length. 


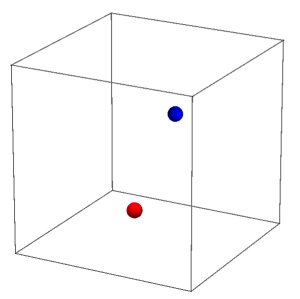

One particle in 1D and one particle in $2 \mathrm{D}$

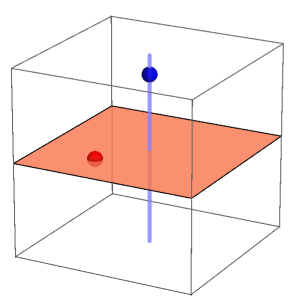

One particle in 3D and one particle in $2 \mathrm{D}$

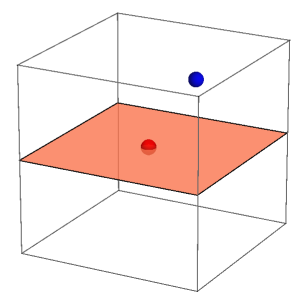

Two particles in 1D and one particle in $2 \mathrm{D}$

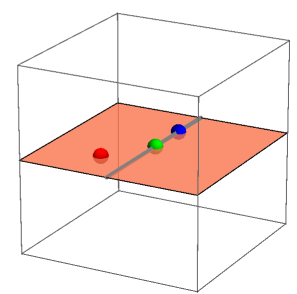

One particle in 3D and one particle in $1 \mathrm{D}$

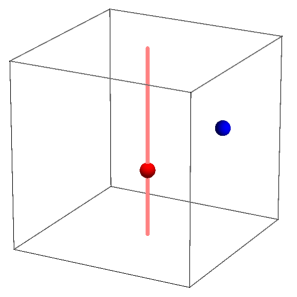

One particle in 1D, one particle in 1D and

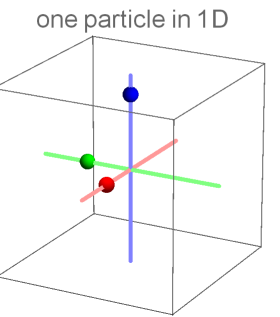

One particle in 2D and one particle in $2 \mathrm{D}$

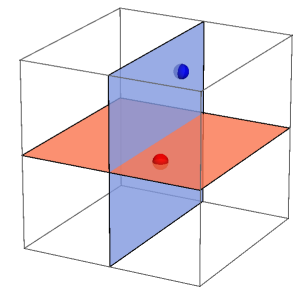

Four particles in 1D

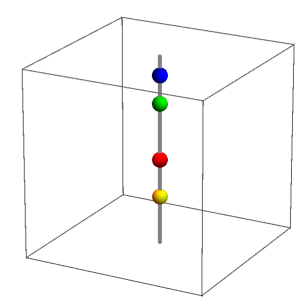

Figure 8.1: All possible cases of $N$ scattering particles in mixed dimensions, whose relative motion is described by exactly three coordinates. The number of coordinates $\sum_{i} D_{i}-D_{\cap}$ is obtained by summing the dimensions $D_{i}$ of subspaces where each particle lives and subtracting the dimension $D_{\cap}$ of the intersection of all subspaces, where the centre of mass coordinates can be eliminated. The first case is the familiar situation of two scattering particles in three dimensions, for which $D_{1}=D_{2}=3$ and $D_{\cap}=3$. The cases involving $D_{i}=0$ are not represented, as they correspond to the trivial problems of fixed scatterers.

Similarly to the dimer case, it is clear that as soon as the system is confined, the trimers' size cannot extend beyond the confinement length $l$. Thus, among the infinity of Efimov trimers, only those which can fit within the confinement region can exist. It would seem natural that the last two Efimov trimers, which are the largest in size, and thus the most sensitive to confinement, would turn into $2 \mathrm{D}$ trimers in the limit of strong confinement. However, the calculations of Levinsen and collaborators show that the situation is slightly more sophisticated. It is the ground-state and first-excited Efimov states which continuously turn into quasi-2D trimers as their binding energy is lowered by tuning the $(3 \mathrm{D})$ scattering length to negative values. These states thus never dissociate into the three-body threshold, unlike in free three-dimensional space (the $a_{-}$point). This happens, however, through avoided crossings between the 3D Efimov trimers and 2D universal trimers, as shown in figure 7.1. The other trimer states are pushed up by these avoided crossings. For strong enough confinement, they do not exist all, and there are just two trimers. For weaker confinement, they appear from the dissociation threshold at values of the scattering length that get closer to the value of $a_{-}$in $3 \mathrm{D}$ as the confinement is reduced. In the limit of weak confinement, one retrieves the free-space Efimov spectrum with many trimers.

The authors of reference [264] conjecture that a similar picture should hold for systems confined in two directions, going from 3D to quasi-1D. Similar crossover behavior may also be expected for the massimbalanced two-component Fermi systems discussed in sections 6.2.2 and 6.2.5 [266].

We also note that the crossover between threedimensional bosonic trimers and their two-dimensional counterparts was also studied in two works [267, 268] in the case when one of the three dimensions is compacted to a radius with periodic boundary conditions. The resulting crossover is qualitatively similar to that of [264].

\section{Mixed dimensions}

Although the Efimov effect is restricted to particles in three dimensions, Yusuke Nishida and Shina Tan have found that Efimov physics can extend to particles moving in subspaces of different dimensions, a situation called mixed dimensions [269] that can be realised by confining only certain particles instead of all three particles. In an article entitled "Liberating Efimov physics from three dimensions" [270], they explained the general arguments to identify such favourable situations.

\subsection{The specificity of $D=3$}

First of all, Yusuke Nishida and Shina Tan gave an intuitive interpretation of the absence of Efimov physics in other dimensions than $D=3$. They argued that a necessary condition for Efimov physics is that the 


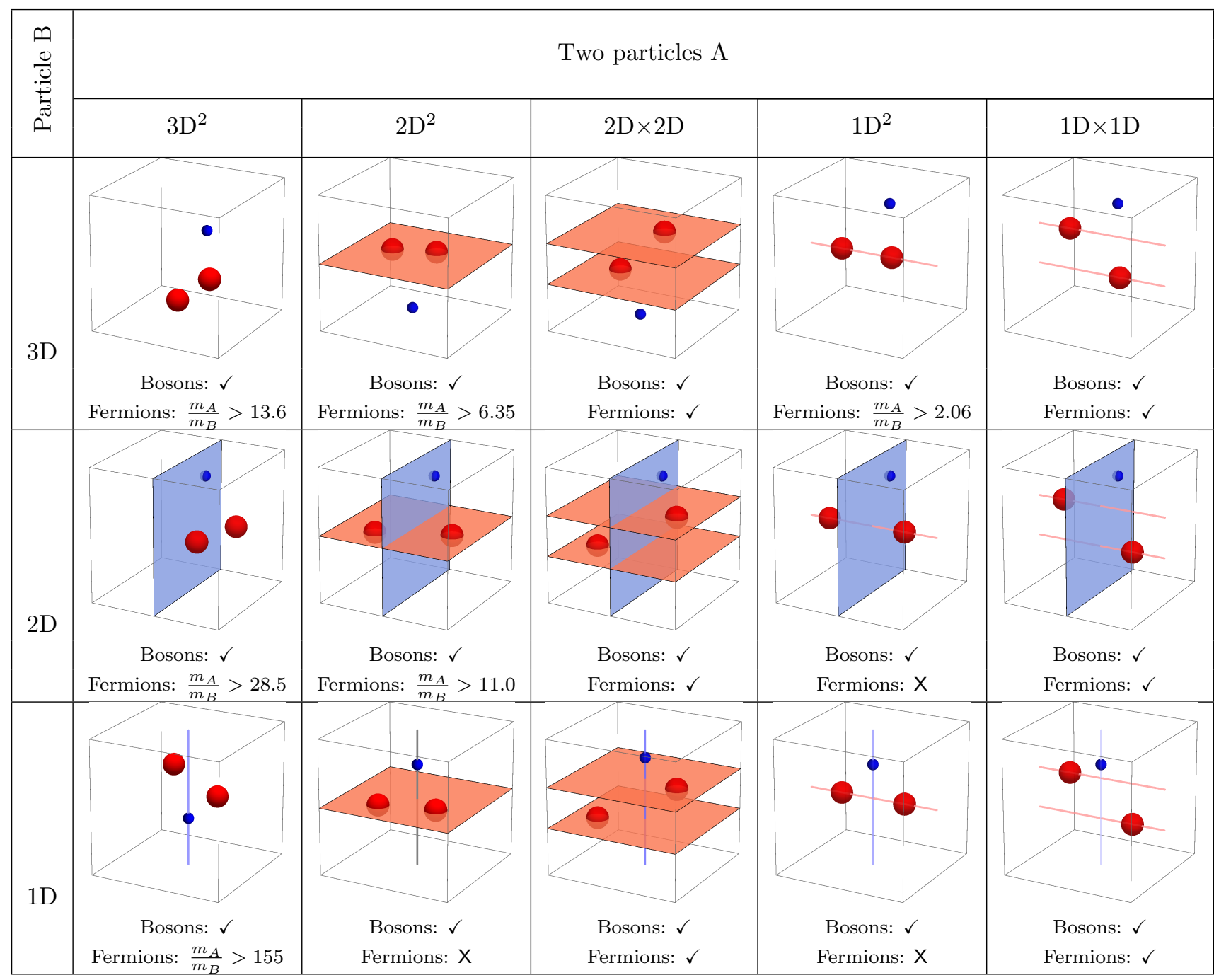

Table 1: Various systems of two particles A of mass $m_{A}$ resontantly interacting with one particle B of mass $m_{B}$ in mixed dimensions, which are expected to exhibit the Efimov effect. These systems are constructed by requiring that at least two two-body subsystems correspond to a case listed in figure 8.1. The first case in the top left corner is the familiar situation where all particles move in three-dimensional space. While the Efimov effect always occurs if the two particles A are identical bosons or distinguishable particles, it may depend on the mass ratio $m_{A} / m_{B}$ if the two particles A are identical (polarised) fermions. For each case, it is indicated whether the Efimov effect occurs $(\checkmark)$ or not $(\mathrm{X})$, or the mass ratio $m_{A} / m_{B}$ beyond which the Efimov effect occurs. In the case of bilayer (3rd column) and biwire (5th column) geometries, the two particles are by construction distinguishable, and their statistics does not matter.

dimensionless two-body wave function at unitarity exhibits a scale-invariant attraction. At short separations $r$, the two-body wave function at unitarity is the singular solution of the Laplace equation. In $D$-dimensions, it is thus

$$
\psi(r) \propto \frac{1}{r^{D-2}}+O\left(r^{4-D}, r^{2}\right) .
$$

For $D \geq 4$, this wave function cannot be normalised due to the divergence at the origin $r=0$, which means that unitarity does not exist in the zero-range limit. Physically, this implies that particles form tight dimers at separations on the order of the range $b$ of the interaction [271]. This tight binding prevents the binding of a third particle at large separations, and thus the
Efimov effect. On the other hand, for $D=2$, the unitarity wave function of equation (9.1) is constant near the origin (this can also be seen from equation (7.6): when $a_{2 \mathrm{D}} \gg b$, the wave function is nearly constant around $r \sim b$ ). This implies that the two particles are effectively non-interacting, which prevents any binding of the particles - indeed, the two-body bound state disappears for $a_{2 \mathrm{D}} \rightarrow \infty$. For $D=1$, the unitarity wave function of equation (9.1) vanishes at the origin as $r$ (this can also be seen from equation (7.1) when $a_{1 \mathrm{D}} \rightarrow 0$ ), which corresponds to a hardcore repulsion, equivalent to the Pauli repulsion (node in the wave function) between non-interacting fermions [256]. This repulsion also prevents particles from binding. There- 
fore, only the case $D=3$ presents a unitary two-body wave function that is scale-invariant and exhibits an attractive effect.

\subsection{Interactions with three relative coordi- nates}

In a second step, Nishida and Tan point out that what matters in general is not the dimension $D=3$ of space itself, but the dimensionality of the relative motion. For two particles moving in the same space, the dimensionality of the relative motion is equal to that of the space, because the locations of the two particles are described by two $D$-vectors, and after elimination of the centre of mass which is also described by a $D$ vector, there remain exactly $D$ coordinates. However, the situation is different for particles moving in subspaces of different dimensions $D_{i}$. The dimensionality is given by the sum of the dimensions of the subspaces in which each particle moves, minus the dimension of the intersection of all sub-spaces, in which the centreof-mass coordinates can be separated from the relative motion. Figure 8.1 shows all the possible combinations of scattering particles in mixed dimensions such that the relative motion is described by exactly three coordinates. In all cases, the equation of relative motion is therefore the same Laplace equation, assuming that the particles interact through an $N$-particle contact interaction specified at unitarity by the scale-invariant boundary condition:

$$
\psi\left(x_{1}, x_{2}, x_{3}\right) \propto \frac{1}{r}+O(r) \text { with } r=\sqrt{x_{1}^{2}+x_{2}^{2}+x_{3}^{2}}
$$

Hence, by analogy with the purely three-dimensional case, one can expect that the addition of an extra particle in these systems will lead the Efimov effect as well. According to Yusuke Nishida and Shina Tan, this is indeed true, although they have postponed the actual demonstration in the general case to a future publication. Nevertheless, they give results for several cases involving two identical particles $\mathrm{A}$ and a particle B. The cases are shown in Table 1.

\subsection{Confinement-induced Efimov effect}

For each case, Nishida and Tan have numerically calculated the scaling strength $\left|s_{0}\right|$ as a function of the mass ratio $m_{A} / m_{B}$. As in the pure $3 \mathrm{D}$ case (see section 6.4 ), $\left|s_{0}\right|$ monotonically increases with the mass ratio, and in the case of fermions cancels at a critical mass ratio, below which the Efimov effect does not occur. The critical mass ratios are given in Table 1. Interestingly, the strength $\left|s_{0}\right|$ also increases when the dimension of space for one or two particles is reduced. Thus confining one or two particles into lower-dimensional spaces makes it easier to observe Efimov states. In particular, in the case of particles including identical fermions for which the mass ratio is not enough to yield Efimov attraction, the Efimov effect may occur by confining one or two particles [272]. Yusuke Nishida and Shina Tan call this effect the confinement-induced Efimov effect.

\subsection{Stable Efimov trimers in bilayer or bi- wire geometries}

Another remarkable situation is the case of particles separated in disjoint subspaces, such as parallel layers [273] or wires, as shown in the third and fifth column of Table 1. Of course, for a two-body contact interaction to take place, these disjoint subspaces must intersect the space of the third particle. This way, both particles can interact with the third particle, and thus the third particle can mediate interaction between the two particles. Yet, the two particles are always spatially separated and cannot come in contact. This has two major consequences.

First, the statistics of the particles does not matter any more, because they can be regarded as distinguishable particles, being with certainty in different locations. Thus, the Pauli repulsion between identical fermions that limits the Efimov effect to sufficiently large mass ratios, does not play a role any more. In such mixed-dimensional settings, fermions always exhibit the Efimov effect.

Second, even though two of the three particles can come in contact, the three particles can never come closer than the separation between the two disjoint subspaces. In systems undergoing loss by threerecombination (such as ultra-cold atoms), this fact can completely suppress the loss, by preventing the three particles from coming all three at distances where recombination occurs. It is thus a clever way to realise Efimov states that are inherently stable, unlike their counterpart in free space.

Since the separation $d$ between the two subspaces constitutes the smallest distance that the three particles can come to, it is the length scale that breaks the discrete scale invariance and determines the groundstate energy and three-body parameter of the trimers. In particular, at unitarity,

$$
\begin{gathered}
E^{(0)}=-\frac{\hbar^{2} \kappa_{*}^{(0) 2}}{2 \mu} \text { and } E^{(n \gg 1)}=-\frac{\hbar^{2} \kappa_{*}^{2}}{2 \mu} e^{-2 n \pi /\left|s_{0}\right|} \\
\text { with } \kappa_{*}^{(0)}=\frac{\alpha_{0}}{d} \text { and } \kappa_{*}=\frac{\alpha}{d}
\end{gathered}
$$

The constants $\alpha_{0}$ and $\alpha$ have been calculated numerically by Yusuke Nishida and Shina Tan for both the bilayer-free $(2 \mathrm{D} \times 2 \mathrm{D} \times 3 \mathrm{D})$ and biwire-free $(1 \mathrm{D} \times 1 \mathrm{D} \times 3 \mathrm{D})$ geometries, for the two mass ratios $40 / 6$ and $6 / 40$. The strength $\left|s_{0}\right|$ is the same as for the single-layer $\left(2 \mathrm{D}^{2} \times 3 \mathrm{D}\right)$ and single-wire $\left(1 \mathrm{D}^{2} \times 3 \mathrm{D}\right)$ geometries, because in the limit of weakly bound Efimov trimers, the separation between the layers or wires is vanishly small compared with the size of the trimers, 
and can be regarded as a single layer or wire in the calculation of $s_{0}$.

Explicit calculations of the trimer energies as a function of the scattering length for bilayer-free and biwire-free geometries were performed by Tao Tin, Peng Zhang, and Wei Zhang [274], using the BornOppenheimer approximation. The authors also calculated the ground-state tetramer energy for the triwirefree geometry. They found that the trimers and tetramers exist in a range of negative and positive scattering lengths, as in the 3D case, and the binding energy reaches a maximum when the scattering length is close to the separation between the layers or wires.

\subsection{Observations with ultra-cold atoms}

The mixed dimension setting has been studied experimentally in only one group so far. The group of Massimo Inguscio and Francesco Minardi in Florence have realised a 2D-3D mixed-dimensional system by confining potassium atoms in the 2D layers of an optical lattice, and let them interact with rubidium atoms [275]. The interactions between the two species can be changed by a magnetic Feshbach resonance. This way, the researchers could explore the variation of the effective mix-dimensional scattering length, and observed through recombination loss spectroscopy the location of mix-dimensional two-body resonances that are required for Efimov physics to set in [276]. However, Efimov features predicted in the preceding sections have not been revealed yet. 


\section{Part IV}

\section{The three-body parameter}

\section{What is the three-body parameter?}

\subsection{In the zero-range theory}

As explained in section 4.1, the three-body parameter is a parameter that needs to be introduced to regularise the zero-range theory of three particles. This parameter can be introduced in several ways depending on the formalism used: a three-body short-range boundary condition or a three-body short-range phase [1], a two- or three-body momentum cut-off [60], or a threebody contact interaction [6]. Therefore, there is not a single formal definition of this parameter, and it can take the form of a length or an energy, etc.

In any case, it is associated with three-body observables, such as the trimer energy or the particle-dimer scattering length. When the three-body parameter is changed, the value of these observables is rescaled accordingly. In the Efimov spectrum as a function of inverse scattering length shown in figure 4.2, this corresponds to a radial rescaling of the curves with respect to the accumulation point at the centre. The following observables (shown in figure 4.2) are often taken as convenient references to characterise the three-body parameter:

1. the binding wave number $\kappa_{*}$ associated with the trimer energy $-\hbar^{2} \kappa_{*}^{2} / m$ at unitarity (the scattering length $a \rightarrow \pm \infty$ ).

2. the dissociation scattering length $a_{-}$at which the trimer vanishes in the three-body scattering threshold.

3. the scattering length $a_{+}$at which the three-body recombination rate has a minimum (for lossy systems).

4. the scattering length $a_{*}$ at which the trimer vanishes at the particle + dimer scattering threshold.

These observables are related to each other by universal relations in the zero-range theory. Namely, for identical bosons [4, 277],

$$
\begin{gathered}
a_{-} \simeq-1.50763 \kappa_{*}^{-1} \\
a_{+} \simeq 0.32 \kappa_{*}^{-1} \\
a_{*} \simeq 0.0707645086901 \kappa_{*}^{-1}
\end{gathered}
$$

Note that because of the discrete scale invariance, these quantities are defined up to a factor $e^{n \pi /\left|s_{0}\right|}$, with $n \in$ $\mathbb{Z}$. In other words, $\kappa_{*}$ and $\kappa_{*} e^{n \pi /\left|s_{0}\right|}$ represent the same three-body parameter.

\subsection{In systems with finite-range interac- tions}

The zero-range theory is of course an idealisation, since in reality interactions do have a finite (i.e. non-zero) range. In reality, the zero-range theory is applicable in the universal window of very large scattering length and very small energy. In this window, the above observables can be used to determine the three-body parameter. In this sense, the three-body parameter, although originally a parameter of the zero-range theory, can also be determined from finite-range calculations or experimental data provided they can access the universal window of highly-excited states or lowenergy scattering. In general, it might be better to talk about the three-body phase, as in equation (4.36), which is a physical quantity independent of the model, and reserve the expression three-body parameter for the parameter of zero-range theories that fixes that phase. Nevertheless, the expression three-body parameter is commonly used in the sense of three-body phase, even in experimental contexts.

In a realistic system, it is often experimentally or computationally difficult to access the universal window corresponding to highly excited trimers. From the first few trimers of the Efimov series of a realistic (finite-range) system, one may extract quantities $\kappa_{*}^{(i)}$, $a_{-}^{(i)}, a_{+}^{(i)}, a_{*}^{(i)}$ for each trimer $i=0,1,2 \ldots$, and consider them as approximate measures of the three-body parameter. Strictly speaking, only in the large $i$ limit do they tend to values representing the three-body parameter (up to a factor $e^{n \pi /\left|s_{0}\right|}$ ), and the universal relations (10.1-10.3) hold only approximately for $\kappa_{*}^{(i)}$, $a_{-}^{(i)}, a_{+}^{(i)}, a_{*}^{(i)}$ with small $i$. On the other hand, the finite-range system quantities $\kappa_{*}^{(i)}, a_{-}^{(i)}, a_{+}^{(i)}, a_{*}^{(i)}$ can help remove the ambiguity over the factor $e^{n \pi /\left|s_{0}\right|}$ in the definition of $\kappa_{*}, a_{-}, a_{+}, a_{*}$. For instance, one can restrict the definition of $\kappa_{*}$ such that $\kappa_{*}\left(e^{\pi /\left|s_{0}\right|}\right)^{i}$ corresponds approximately to the binding wave number $\kappa_{*}^{(i)}$ of the $i$-th Efimov state. However, this definition still depends on what is considered to be the first Efimov state. For simplicity, and in view of the recent definitions of "Efimov state" (see section 4.5), we will always label the ground-state trimer as the first Efimov state $i=0$.

\subsection{In systems with loss}

When the Efimov effect occurs in systems supporting several two-body bound states, the Efimov states are trimer resonances that can decay by recombining into a lower two-body bound state scattering off a third particle. In the zero-range theory, such lower two-body bound states are not present, yet Eric Braaten, HansWerner Hammer, and Masaoki Kusunoki have shown that their combined effect can be described in the zerorange theory by introducing an inelasiticity parameter $\eta>0[63,4,120]$. Physically, $e^{-4 \eta}$ represents the prob- 
ability of an incoming hyper-radial flow to be reflected back to large hyper-radii, the rest being lost at short distance by recombination. Formally, this amounts to giving the three-body parameter a complex value $\kappa_{*} e^{i 2 \eta /\left|s_{0}\right|}$. This makes the energy of the Efimov states complex, $E+\frac{i}{2} \Gamma$, where the imaginary part corresponds to the energy width associated with their finite lifetime. In the limit of small $\eta$, one finds $\Gamma=\frac{4 \eta}{\left|s_{0}\right|} E$.

\section{What sets the three-body parameter?}

A natural question that arises is what in the microscopic details of the finite-range interactions of real systems determines the three-body parameter. At first glance, two different views can be proposed:

1. The three-body parameter is roughly determined by the range of interaction The first view is that the scale of the three-body parameter is roughly determined by the range $b$ of the interaction, i.e. the length beyond which the interaction can be taken as null or negligible. Indeed, this range constitutes the length scale below which the zero-range theory ceases to be valid. It is when the three particles approach this range that the three-body boundary condition determining the three-body parameter is set. It is therefore a natural guess that the resulting three-body parameter, expressed as a length scale, is on the order of this range.

This idea is supported by the early work of Llewellyn H. Thomas [18], although the Efimov effect was not known at the time. In this work, it was found that the ground state of the three-body problem depends on the range of the interaction. Namely, its energy becomes deeper as the range of interaction is reduced; in the limit of zero-range interaction, the energy goes to minus infinity, in other words the spectrum is unbound from below, a phenomenon later known as the Thomas collapse. Llewellyn Thomas used this finding to estimate the triton binding energy from the range of nuclear forces.

2. The three-body parameter can take any value The second view is that the three-body parameter is a threebody boundary condition for the free wave, in the same way as the two-body scattering length being a twobody boundary condition for the free wave - see the Bethe-Peierls condition (4.7). It is known that the scattering length is in general difficult to predict from the details of the two-body interaction. For a purely repulsive interaction, the scattering length is on the order of the interaction range, but for an attractive interaction, it can be very different. Although on average (over many interaction potentials) the scattering length is on the order of the interaction range, it may be much larger as well as positive or negative [278], and one is often required to solve the two-body Schrödinger equation to obtain the value of the scattering length for a given interaction potential. Especially for interaction potentials with a deep well, the scattering wave function accumulates a large phase inside the well, and a minute change in the short-range details of the potential can completely change the value of the scattering length.

By analogy, in the three-body problem at unitarity, one can view the three-body body parameter as the result of the short-range boundary condition for the hyper-radial problem with the Efimov attractive potential of equation (4.33). The short-range boundary condition arises from the complicated three-body dynamics at short range where all hyper-angular channels are coupled. To get some insight, though, one can model this dynamics by an effective short-range hyperradial potential.

As in the two-body case, if this short-range potential is purely repulsive (such as a hard wall at $R=R_{0}$ ) the three-body parameter is set by the range of the repulsion. On the other hand, if the short-range potential is attractive, it can take very different values. For instance, if an attractive $-1 / R^{6}$ potential well (van der Waals type) is used as a short-range boundary condition, one finds that the three-body parameter can take any value with substantial probability - see the left panel of figure 11.1. From these simple calculations, one concludes that in general the three-body parameter, like the twobody scattering lengh, can take on any value and is sensitive to the short-range details of the interaction.

The above two views 1 and 2 can be conciled by saying that in general the three-body parameter can take any value depending on the short-range details of the interaction, but on average (over many interaction potentials) it scales with the range of the interaction.

While it is true that the three-body parameter may in general take any value, there are some notable cases presented in the following sections for which it is simply related to a length scale of the two-body interaction.

\subsection{First calculations}

There have been many calculations of the three-body problem with finite-range interactions near unitarity, from which one can extract the Efimov three-body parameter, at least approximately. For example, the work of Moszkowski and co-workers [279] has investigated, for many shapes of two-body potential, the strength required to bind three particles with respect to the strength required to bind two particles - such a calculation is equivalent to the determination of $a_{-}^{(0)}$, which is an approximate measure of the three-body parameter. Their calculation indicates that the strength ratio could vary from $2 / 3$ to 1 , although it is often close to 0.8 , which suggests a nominal value for the three- 

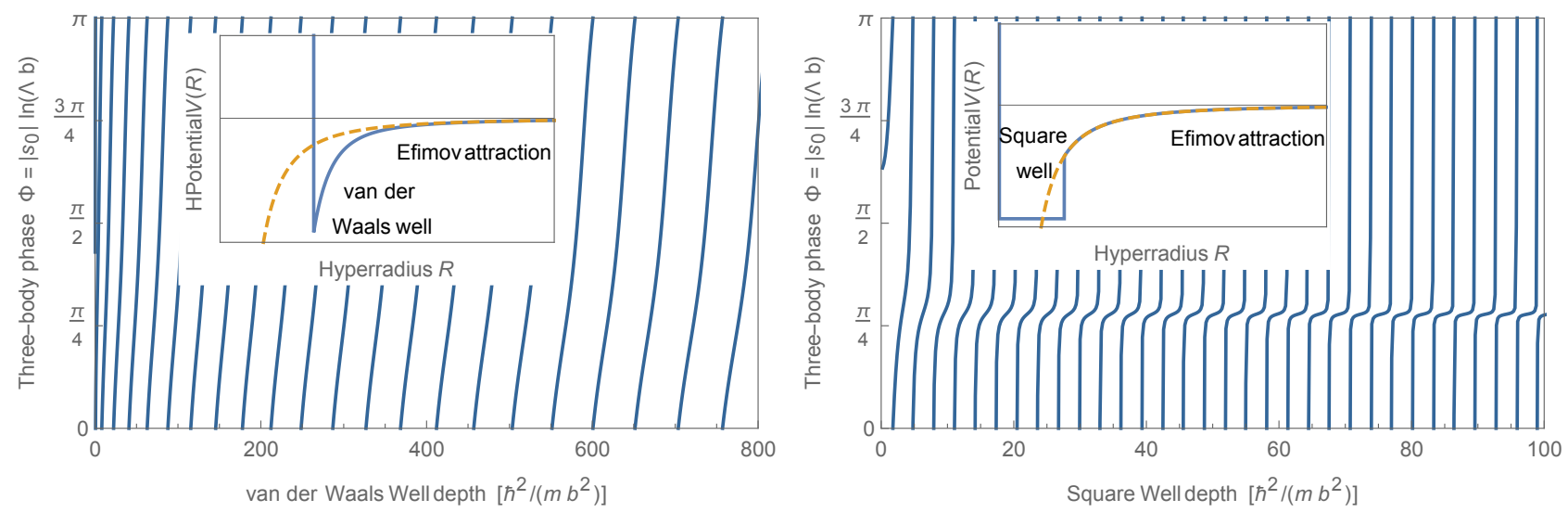

Figure 11.1: Three-body phase of equation (4.36) for the solution of the hyper-radial equation (4.31), where the hyper-radial potential $V(R)$ (represented in the insets) consists of the Efimov long-range attraction $V_{0}$ of equation (4.33) at large distance, and an attractive well of range $b$ setting the boundary condition at short distance. Left panel: the attractive well is a van der Waals potential with a van der Waals length $b$, and a repulsive wall at some variable hyper-radius $R_{0}$, i.e. $V(R)=V_{0}(R)-(2 b)^{4} / R^{6}$ for $R>R_{0}$ and $V(R)=\infty$ for $R \leq R_{0}$. Right panel: the attractive well is a square-well potential of radius $b$ and variable depth. The reference length scale $\Lambda_{0}^{-1}$ of equation (4.36) has been set to $b$.

body parameter, supporting view 1 .

Later, motivated by the experimental development of Efimov physics in ultra-cold atomic gases, José D'Incao and co-workers [280] investigated more specifically the variation of the three-body parameter from one two-body resonance to another, as the strength of the same two-body potential is increased (the Efimov states in this case are resonances -see the end of section 4.5). They concluded that the value of the threebody parameter changes significantly, and is further modified by the presence of a three-body force, supporting view 2 .

\subsection{Van der Waals universality}

\subsubsection{Three identical bosons}

Until Efimov states could be observed in ultra-cold atomic gases, there was no experimental data directly related to the three-body parameter. From 2006 onwards, quantities such as $\kappa_{*}, a_{-}, a_{+}$, and $a_{*}$ could be measured around different resonances in various atomic species (see section 4.7.2), at least for the ground Efimov state and in some cases the first excited Efimov state, thus providing some experimental estimate of the three-body parameter. Surprisingly, the obtained values $[281,37]$ were not randomly distributed over an Efimov log-period [1, $\left.e^{\pi / s_{0}} \approx 22.7\right]$, but instead showed a strong correlation with the range of the atomic interaction, taken to be the van der Waals length ${ }^{15}$,

$$
\ell_{\mathrm{vdW}}=\frac{1}{2}\left(2 \mu C_{6} / \hbar^{2}\right)^{1 / 4},
$$

\footnotetext{
${ }^{15}$ Not to be confused with the van der Waals radius $r_{W}$ that accounts for the finite size of atoms and molecules in the equation of state of gases.
}

associated with the van der Waals tail $-C_{6} / r^{6}$ of the interaction betwen two neutral atoms of reduced mass $\mu$ [126]. The correlation of experimental results for $a_{-}$ for identical bosons is shown in figure 11.2. It gives on average:

$$
a_{-}=-(8.9 \pm 1.8) \ell_{\mathrm{vdw}} .
$$

This average value is obtained from values of $a_{-}^{(n)} e^{-n \pi / s_{0}}$ for the ground-state $(n=0)$ and firstexcited $(n=1)$ Efimov states. The stated uncertainty is two standard deviations of the data; all experimental data fall into this range.

The values obtained from the ground-state resonances are expected to deviate (by up to $25 \%$ ) from the exact three-body parameter. These values alone give:

$$
a_{-}^{(0)}=-(9.1 \pm 1.5) \ell_{\mathrm{vdW}} .
$$

The first-excited-state resonances are expected to be closer to the exact three-body parameter (within a few percent). There are currently two experimental values for such resonances. One is for a resonance in caesium133 at a magnetic field around 800 G [40],

$$
a_{-}^{(1)} e^{-\pi /\left|s_{0}\right|}=-8.8(4) \ell_{\mathrm{vdW}} .
$$

The other comes from a resonance at around $900 \mathrm{G}$ in lithium-6 experiments [242, 239, 240, 75, 78], which involve three distinguishable fermions behaving like identical bosons. A recent and thorough analysis of the data [248], including thermal corrections, gives the value

$$
a_{-}^{(1)} e^{-\pi /\left|s_{0}\right|}=-7.11(6) \ell_{\mathrm{vdW}} .
$$

All these results indicate that within an error of $20 \%$, the three-body parameter is universally determined by the van der Waals length, with $a_{-} \approx-9 \ell_{\mathrm{vdw}}$. 


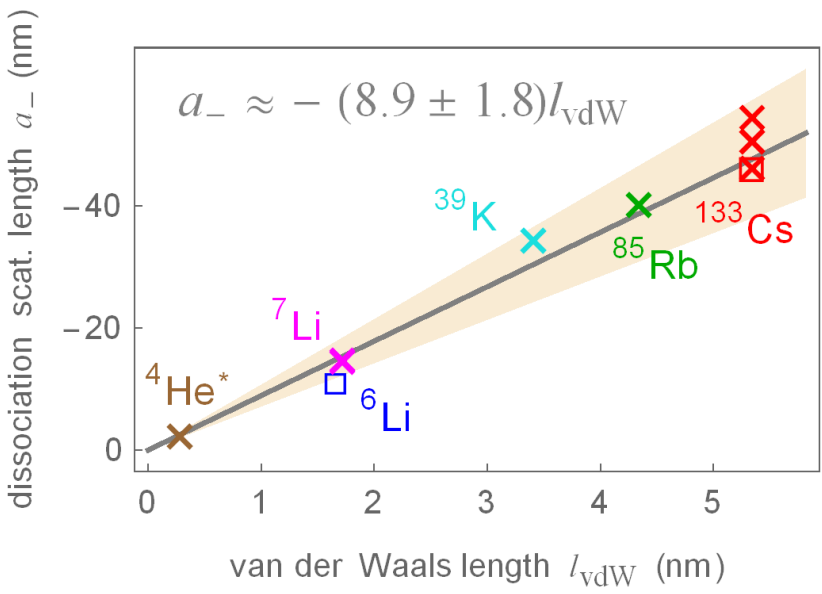

Figure 11.2: Van der Waals universality of the threebody parameter observed in ultra-cold atomic gases for caesium-133 [37, 32, 40], rubidium-85 [282], potassium39 [139], lithium-7 [143, 140, 281], and metastable helium-4 [283]. For potassium-39, the data corresponds to a point originally assigned to a four-body resonance [141]. Also shown are the results [248] of lithium6 experiments [241,242, 238, 239, 240, 75, 78, 248], which involve three distinguishable fermions behaving like identical bosons, except that they have three different scattering lengths. The value of $a_{-}$for these experiments is estimated by the formula $a_{-}^{(n)} e^{-n \pi / s_{0}}$. Results for $n=0$ (ground state) are shown by crosses, whereas results for $n=1$ (excited state) are shown by squares. A linear fit of the results is plotted, along with a shaded area indicating two standard deviations.

This remarkable experimental finding triggered theoretical activity to understand its origin. The first proposed explanation [284] invoked quantum reflection of the three-body wave function in the region where atoms undergo van der Waals attraction. The argument supporting this idea is based on the calculation of the three-body phase from a hyper-radial potential which consists of an Efimov attraction at large distance and a deep square well potential at short distance (approximating the effects of the two-body van der Waals attraction). It is represented in the right panel of figure 11.1. For deep wells, the three-body phase appears to stabilise around a well-defined value, close to $\pi / 4$. However, the stability of the three-body phase turns out to be a peculiarity of the square well approximation. As we discussed previously, when the square well in the hyper-radius potential is replaced by a van der Waals attraction, the three-body phase can take different values with significant probability - see the left panel of figure 11.1. Nevertheless, the idea of quantum reflection looked compelling and prompted theorists to check thoroughly the physics of three particles interacting via deeply attractive van der Waals potentials.

The work of Jia Wang and co-workers [38] presented the first exact calculation, using the adiabatic hyperspherical representation. In this method, the threebody wave function is expressed as a linear combination of products of hyper-radial and hyper-angular wave functions $\Psi=\sum_{n} F_{n}(R) \Phi_{n}(\Omega ; R)$, where the hyperradius $R$ characterises the size of the three-body system and is defined ${ }^{16}$ as $R^{2}=\frac{2}{3}\left(r_{12}^{2}+r_{23}^{2}+r_{31}^{2}\right)$, and $\Omega$ denotes the set of remaining coordinates describing the geometry of the three-body system, which are called hyper-angles. Note that this form becomes separable only for the zero-range interaction at unitarity, see equation (4.27). For finite-range interactions such as the van der Waals type, the hyper-radius $R$ is coupled to the hyper-angles $\Omega$ at short distance. Hyperangular wave functions $\Phi_{n}$ are thus calculated to obtain hyper-radial potentials $W_{n}(R)$, as well as coupling terms $W_{n n^{\prime}}(R)$, which lead to a set of coupled equations for the hyper-radial motion:

$$
\left(-\frac{d^{2}}{d R^{2}}+W_{n}(R)-E\right) F_{n}(R)+\sum_{n^{\prime} \neq n} W_{n, n^{\prime}}(R) F_{n^{\prime}}(R)=0
$$

where $R$ is expressed in units of van der Waals length $\ell_{\mathrm{vdw}}$ and the potentials $W_{n}, W_{n, n^{\prime}}$ is expressed in units of van der Waals energy $E_{\mathrm{vdW}}=\frac{\hbar^{2}}{m \ell_{\mathrm{vdW}}^{2}}$. These hyperradial equations uncouple at large hyper-radius, and one of them features a hyper-radial potential $W_{0}(R)$ that asymptotes to the Efimov attraction $V_{0}(R)$ of equation (4.33). Solving the coupled equations (11.5) for various two-body interactions with a van der Waals tail, Jia Wang and co-workers found that the threebody parameter is essentially determined by the van der Waals tail and relatively insensitive to other details. In the limit of deep van der Waals interactions, they found:

$$
\begin{aligned}
& \kappa_{*}^{(0)}=(0.21 \pm 0.01) / \ell_{\mathrm{vdW}} \\
& a_{-}^{(0)}=-(10.70 \pm 0.35) \ell_{\mathrm{vdW}}
\end{aligned}
$$

which is close to ultra-cold atom observations given in figure 11.2 and equation (11.2). For shallower potentials for which the van der Waals character is less pronounced, they found slightly larger values of $\kappa_{*}^{(0)}$ and $a_{-}^{(0)}$. These values were recently confirmed in Refs. [285, 286] for shallow van der Waals potentials with a repulsive core and supporting only one two-body bound state, giving:

$$
\begin{aligned}
& \kappa_{*}^{(0)}=(0.226 \pm 0.004) / \ell_{\mathrm{vdW}} \\
& a_{-}^{(0)}=-(9.69 \pm 0.20) \ell_{\mathrm{vdW}}
\end{aligned}
$$

For all van der Waals potentials investigated so far, deep or shallow, with or without a repulsive core,

\footnotetext{
${ }^{16}$ Note that the authors of reference [38] use a different convention for the definition of the hyper-radius, namely $R^{2}=$ $\frac{1}{\sqrt{3}}\left(r_{12}^{2}+r_{23}^{2}+r_{31}^{2}\right)$. Here, we use a definition that is consistent with the one used in section 4.1 - see equation (4.24).
} 
the values of $a_{-}^{(0)} / \ell_{\mathrm{vdW}}$ are comprised in the range $[-11.2,-8.3]$, i.e. $-9.73 \pm 15 \%$. This theoretical value is often cited in the literature, as it agrees well with the experimental value of equation (11.2). However it is more relevant to shallow potentials, and the value in equation (11.7) should be taken as the universal value relevant to interactions dominated by their van der Waals tail. The more important deviation between this value and the experimental value of equation (11.2) suggests that other physics beyond the van der Waals universality may contribute to the three-body parameter of atoms close to a broad magnetic Feshbach resonance [248].

Three-body repulsion Jia Wang and co-workers looked for a simple reason for this universality in their calculated hyper-radial potentials. They found that the potential $W_{0}(R)$ exhibits a steep repulsive barrier at a hyper-radius $R \approx 2 \ell_{\mathrm{vdw}}$, instead of a strong attraction as one would naïvely expect from the van der Waals attraction. Furthermore, they found that this repulsive barrier is always present, irrespective of the presence or absence of a repulsive core ${ }^{17}$ in the twobody potential, and in the limit of deeply attractive potentials has a seemingly universal form that depends only on the van der Waals tail of the potential - see the grey curves in the top panel of Fig. 11.3. This observation alone cannot explain their results, because the potential $W_{0}(R)$ is strongly coupled to other channels in the region of the repulsive barrier. Solving the coupled equations, Jia Wang and co-workers found that this repulsive barrier is actually enhanced by the hyper-radial couplings $W_{n n \prime}$ and effectively suppresses the probability of finding the three particles within a hyper-radius $R<2 \ell_{\mathrm{vdW}}$. The authors confirmed that imposing such a steep barrier by hand in the potential $W_{0}(R)$ alone sets the three-body phase to a value in good agreement with their exact results (11.6-11.7). The repulsive barrier also explains why the results do not depend much on the interatomic three-body forces, such as the Axilrod-Teller potential [289]: the repulsive barrier prevents the three atoms from coming to the short distances where they would experience the three-body force.

The three-body repulsive barrier was confirmed in a different manner by Emiko Hiyama and Masayasu Kamimura [285] from exact three-body calculations with various helium potentials. Using the Gaussian expansion method to solve the three-body problem, they obtained the energies $E$ and the three-body wave functions of trimers at unitarity. Integrating the squared wave function over hyper-angles to obtain the integrated hyper-radial density $\rho(R)$ gives the effective hyper-radial wave function $F(R)=R \rho(R)^{1 / 2}$. This

\footnotetext{
${ }^{17}$ Shortly after the findings of Jia Wang and co-workers, a work [288] proposed that the three-body repulsion originates from the repulsive core in the two-body potential. However, this statement is not consistent with the results of Jia Wang and co-workers.
}

can be converted into the effective hyper-radial potential

$$
U(R)=\frac{1}{F(R)} \frac{d^{2}}{d R^{2}} F(R)+E
$$

assuming that $F(R)$ satisfies a single Schrödinger equation akin to equation (11.5). Hiyama and Kamimura found that for all the helium pairwise potentials, both the ground and first-excited trimers give the same potential $U(R)$ in the van der Waals region and it exhibits the universal repulsive barrier - see the green curve in the top panel of figure 11.3. This results in $a_{-}^{(0)}=-9.78(1) \ell_{\mathrm{vdW}}$ for all the helium potentials, in agreement with the calculations of Refs. [38, 286] for shallow Lennard-Jones potentials.

The calculations of Refs. [38, 285] thus indicate that the origin of the van der Waals universality of the threebody parameter is a three-body repulsion rather than quantum reflection. Jia Wang and co-workers noted that this repulsion originates from the suppression of probability to find two atoms at short separation. This suppression of probability is due, as in classical mechanics, to the acceleration of the relative motion by the attractive potential, which makes the two atoms spend little time at short separation. This suppression squeezes the three-body wave function, which results in an increase of kinetic energy that is responsible for the repulsive barrier.

Connection with two-body physics The work of reference [39] confirmed the interpretation of Jia Wang and co-workers [38], and showed that this increase of kinetic energy is physically related to a deformation of the three-body system from its configuration at large separations to shorter hyper-radii. At large hyperradius, the three-body configuration is independent of the hyper-radius, and distributed according to the Efimov hyper-angular wave function,

$$
\Phi_{0}^{(Z R)}(\Omega)=\sum_{i=1}^{3} \frac{\phi_{0}\left(\alpha_{i}\right)}{\sin 2 \alpha_{i}},
$$

where $\phi_{0}$ is given by equation (4.28) and $\alpha_{i}$ denote the hyper-angles $\alpha$ for the three possible Jacobi sets of coordinates given by equations (4.9-4.10). As mentioned in section 4.5.2, the most probable configurations have an elongated-triangle geometry: two particles are close and one is farther away. However, at hyper-radii on the order of the van der Waals length, the suppression of two-body probability forces the system to adopt an equilateral configuration - see the bottom panel of figure 11.3. This change of configuration can be seen in the hyper-radius dependence of the hyper-angular wave function $\Phi_{0}$, and the kinetic energy associated with this deformation is described by the non-adiabatic term

$$
Q_{00}(R)=\int d \Omega\left|\frac{d \Phi_{0}}{d R}\right|^{2}
$$



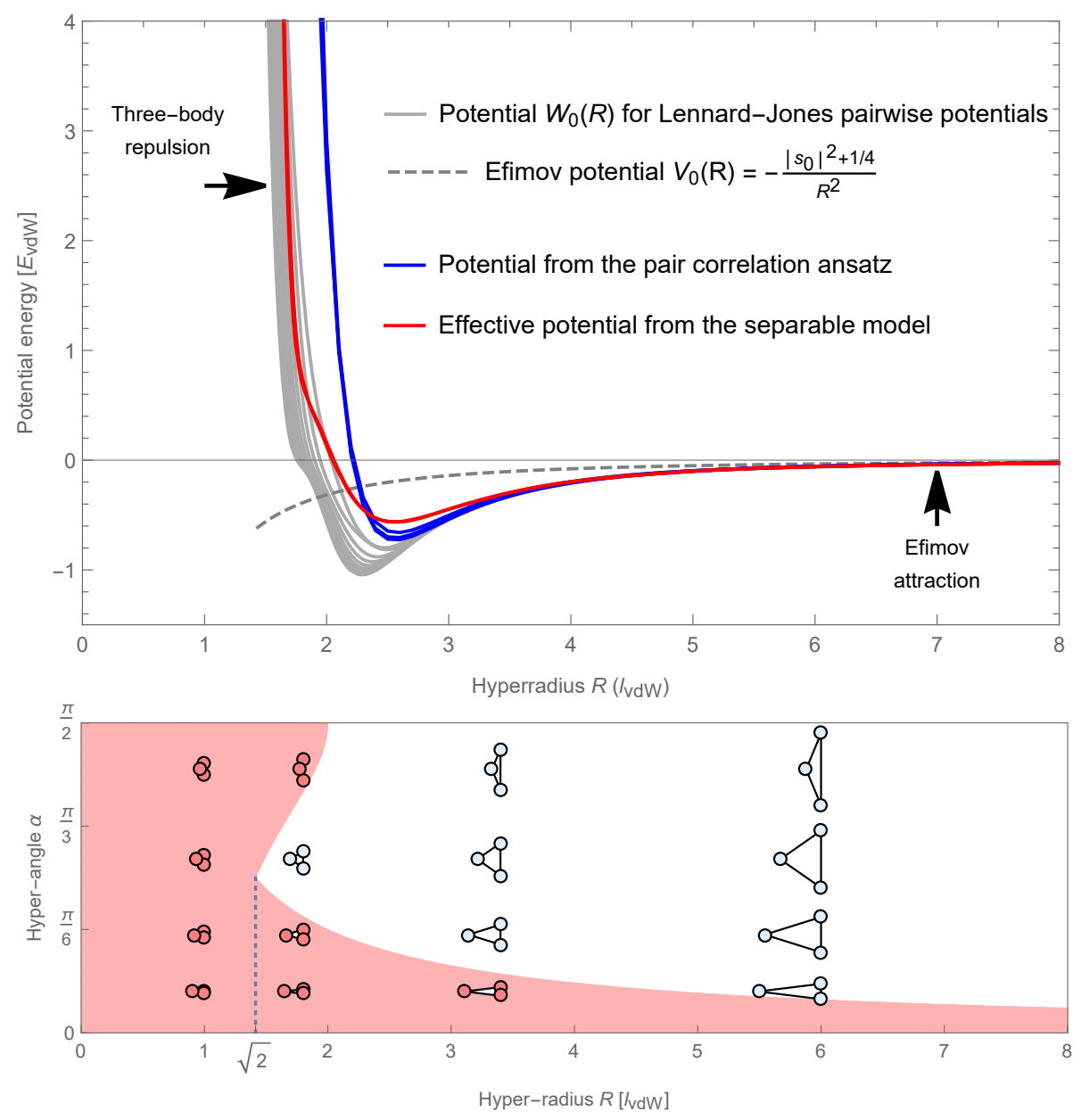

Figure 11.3: Top: Potential energy for three bosons resonantly interacting via two-body van der Waals interactions as a function of their hyper-radius. The grey curves represent the diagonal potential $W_{0}(R)$ obtained in reference [38] for the channel that asymptotes to the Efimov attraction $V_{0}(R)$ (shown in dashes). The different curves correspond to calculations for two-body potentials (6-12 Lennard-Jones type [287]) of different depths, supporting from 2 to $10 \mathrm{~s}$-wave two-body bound states (from top to bottom). The green curves correspond to the effective potential (11.10) obtained from the ground and first-excited helium trimer wave function calculated in reference [285] with various helium potentials scaled to unitarity. They overlap within their thickness. The blue curves represent the potential obtained from the pair ansatz (11.13) of reference [82]. They correspond to the zero-energy two-body wave functions $\varphi(r)$ for Lennard-Jones potentials supporting 2 to $5 s$-wave bound states. The red curves represent the effective hyper-radial potential (11.10) obtained from the separable model of reference [82] given by equation (11.15). They correspond to the zero-energy two-body wave functions $\varphi(r)$ for Lennard-Jones potentials supporting 3 and $7 s$-wave bound states.

Bottom: Configuration space of the three-boson system as a function of the hyper-radius and one hyper-angle, for a right angle between the two Jacobi vectors.. A few configurations are shown, where bosons are represented by circles with a diameter equal to the van der Waals length $\ell_{\mathrm{vdw}}$. The region of configurations for which at least two atoms are within the distance $\ell_{\mathrm{vdw}}$ from each other (i.e. circles touching each other) is shaded in pink. These configurations have near-zero probability due to the two-body suppression induced by the van der Waals attraction - see figure 11.4. Below the hyper-radius $R=2 \ell_{\mathrm{vdw}}$, configurations therefore have to deform to a nearly equilateral configuration. The kinetic energy cost associated with this deformation results in the repulsive barrier of the top panel.

contained in $W_{0}(R)$. As the three-body system probes shorter hyper-radii, the deformational energy due to the two-body suppression arises as a repulsive barrier in $W_{0}(R)$. To support this interpretation, the authors of reference [82] presented two models built on the van der Waals two-body suppression, that lead to the universal three-body repulsion.

The first model consists in making the following ansatz for the hyper-angular wave function,

$$
\Phi_{0}(\Omega ; R)=\Phi_{0}^{(Z R)}(\Omega) \times \varphi\left(r_{12}\right) \varphi\left(r_{23}\right) \varphi\left(r_{31}\right),
$$

i.e. the hyper-angular wavefunction $\Phi_{0}^{(Z R)}$ from the 


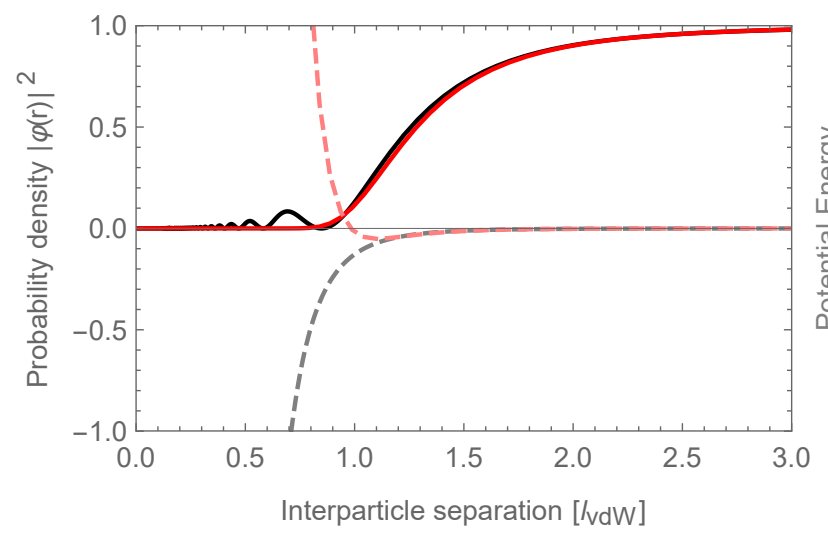

Figure 11.4: Two-body radial probability density $|\varphi(r)|^{2}$ at unitarity $(a \approx \infty)$ as a function of interparticle separation $r$, for a deep van der Waals potential (black, obtained from equation (11.14)) and for a shallow van der Waals potential with a repulsive core (red). The corresponding potentials are shown in dashed curves. In both cases, the probability density is suppressed for $r \lesssim \ell_{\mathrm{vdW}}$. In the first case, this is due the acceleration in the attractive van der Waals potential. In the second case, this is due to the repulsive core. The density profile is nearly the same for $r \gtrsim \ell_{\mathrm{vdW}}$ since both potentials have the van der Waals form in this region.

zero-range theory is multiplied by a two-body correlation function $\varphi$ for all three pairs. This two-body correlation is taken to be the two-body radial wave function $\varphi(r)=r \psi(r)$ at zero-energy for a potential at unitarity with a van der Waals tail. This pair correlation ansatz describes the suppression of two-body probability in a simple fashion, and can be used to calculate the hyper-radial potential $W_{0}(R)$.

The two-body radial wave function $\varphi(r)$ is known to have a universal form in the van der Waals region:

$$
\varphi(r) \underset{r \gtrsim \ell_{\mathrm{vdW}}}{=} \Gamma(5 / 4) \sqrt{x} J_{\frac{1}{4}}\left(2 x^{-2}\right)
$$

where $x=r / \ell_{\mathrm{vdw}}$ and $\Gamma$ and $J_{\alpha}$ denote the gamma and Bessel functions. At shorter distances $r \ll \ell_{\mathrm{vdW}}$ ,$\varphi(r)$ has a potential-dependent form and vanishes at $r=0$. The number of nodes in $\varphi(r)$ corresponds to the number of $s$-wave two-body bound states supported by the potential. The probability density $|\varphi(r)|^{2}$ is plotted as a solid black curve in figure 11.4.

The authors of reference [39] found that the resulting hyper-radial potential $W_{0}(R)$ exhibits a repulsive barrier similar to that of reference [38] - see the blue curves in the top panel of figure 11.3. The barrier is due to the large value of the non-adiabatic term $Q_{00}$ of equation (11.12), confirming the deformation scenario. The repulsive barrier does not depend much on the short-range form of the potential nor its number of bound states, as long as the two-body probability is sufficiently suppressed at short-distance, i.e. $\varphi(r)$ has most of its amplitude in the van der Waals region where it has the universal form (11.14). This occurs for most physical potentials, which feature either a strongly repulsive core or a deep well that reduces the short-distance probability - see figure 11.4.

In the second model of reference [39], the two-body suppression is introduced through a separable representation of two-body van der Waals potentials. Any local potential $V$ can be represented as a superposition of non-local separable potentials, a representation introduced by Ernst, Shakin and Thaler (EST) [290]. Truncating this representation to a single separable potential $\hat{V}$ gives an approximation of the original potential $V$ that reproduces one of its eigenstates $|\psi\rangle$ exactly at a chosen energy, and other eigenstates approximately around that energy. The separable potential $\hat{V}$ is explicitly constructed from that eigenstate as

$$
\hat{V}=\frac{1}{\langle\psi|V| \psi\rangle} V|\psi\rangle\langle\psi| V .
$$

From this expression, one can easily check that the action of $\hat{V}$ and $V$ onto $|\psi\rangle$ is the same. Choosing again $|\psi\rangle$ to be the zero-energy scattering state, the resulting separable potential reproduces that state exactly by construction, and therefore the two-body suppression at low energy. Solving the three-body problem with this separable potential (see Appendix for details), constructed from a $\varphi(r)$ that is dominated by the van der Waals form (11.14), gives a three-body parameter that is consistent with the results of Jia Wang and co-workers [38]. In the limit of a deep van der Waals potential $V$, i.e. when $\varphi(r)$ tends to the van der Waals form (11.14), the authors find

$$
\begin{gathered}
\kappa_{*}^{(0)}=0.187(1) \ell_{\mathrm{vdW}}^{-1} \\
a_{-}^{(0)}=-10.86(1) \ell_{\mathrm{vdW}},
\end{gathered}
$$

in fair agreement with equations (11.6-11.7). Furthermore, the authors integrated the obtained threebody probability density over the hyper-angles to obtain a hyper-radial wave function, and converted that wave function into an effective hyper-radial potential through equation (11.10). The resulting potential is shown by the red curves in the top panel of figure 11.3 and compares well with the potentials of reference [38] and [285].

These results show that the van der Waals universality of the three-body parameter is a consequence of the van der Waals two-body universality given by equation (11.14).

\subsubsection{2 bosons +1 particle}

The van der Waals universality extends to systems of different particles, such as heteronuclear atomic systems. The physics is richer because the particles may have different masses, different quantum statistics, and 
the interactions may have different van der Waals lengths in addition to different scattering lengths. The case of two identical bosons $A$ of mass $m_{A}$ and one particle $X$ of mass $m_{X}$ was theoretically studied by Yujun Wang and co-workers in reference [233] using 612 Lennard-Jones potentials [287] $V_{A A}$ and $V_{A X}$. In this case, as discussed in section 6 , there are two scattering lengths $a_{A A}$ and $a_{A X}$ and two limits for which the Efimov effect occurs: $a_{A X} \rightarrow \pm \infty$ with $a_{A A}$ finite, and $a_{A X}, a_{A A} \rightarrow \pm \infty$. Accordingly, for these two Efimov regimes, there are two geometric scaling strengths, respectively $s_{0}$ and $s_{0}^{*}$ (solutions of equations (6.8) and (6.7)), and two three-body parameters, respectively $\kappa$ and $\kappa^{*}$. The authors of reference [233] found that, for a given mass ratio $m_{A} / m_{X}$ between $A$ and $X$, these three-body parameters are determined solely from the van der Waals lengths $\ell_{\mathrm{vdW}, \mathrm{AA}}$ and $\ell_{\mathrm{vdW}, \mathrm{Ax}}$ (as well as $a_{A A}$ for the first Efimov regime), and do not depend upon other short-range details, as in the case of three identical bosons. Nevertheless, they found that this van der Waals universality is explained differently for the Efimov-favoured limit (the limit of large mass ratio $m_{A} / m_{X}$, i.e. two heavy bosons and one light particle) and the Efimov-unfavoured limit (the limit of small mass ratio $m_{A} / m_{X}$, i.e. two light bosons and one heavy particle).

In the Efimov-unfavoured limit, the two Efimov regimes are well separated. The first Efimov regime corresponds to a family of bound states in a hyperradial potential (that asymptotes to the dissociation threshold $A X+A$ for $a_{A X}>0$ ), whereas the second Efimov regime corresponds to a family of bound states in another hyper-radial potential (that asymptotes to the dissociation threshold $A A+X$ for $a_{A A}>0$ ). The authors of reference [233] found that the hyper-radial potential for the first Efimov regime has a universal form for a fixed $\ell_{\mathrm{vdW}, \mathrm{AA}}$ and $\ell_{\mathrm{vdW}, \mathrm{Ax}}$ when it is expressed in units of $a_{A A}$, while the hyper-radial potential for the second Efimov regime has a universal form for a fixed $\ell_{\mathrm{vdW}, \mathrm{AA}}$ and $\ell_{\mathrm{vdW}, \mathrm{Ax}}$, which is independent of $a_{A A}$. This form exhibits the Efimov attraction at large distance, and a short-range repulsion that is similar to that of three identical bosons. This three-body repulsion presumably follows from the same two-body induced deformation mechanism explained in the previous section.

In the Efimov-favoured limit, on the other hand, the hyper-radial repulsion picture becomes inadequate to understand the three-body parameter: the repulsive barrier in the hyper-radial potential featuring the Efimov attraction goes to shorter and shorter distances and becomes irrelevant, while the couplings to other channels become increasingly strong. The authors of reference [233] found that the Born-Oppenheimer picture is better suited for this situation. This picture consists in considering the two heavy bosons as slow, and take their relative coordinate $r$ as the adiabatic variable instead of the hyper-radius $R$. As the two bosons slowly move, the light particle is assumed to adiabatically follow a wave function $\Phi(\vec{\rho} ; r)$ that is calculated at each separation $r$ of the two bosons. The three-body wave function is therefore

$$
\Psi_{B O}(r, \vec{\rho})=F(r) \Phi(\vec{\rho} ; r)
$$

The determination of $\Phi(\vec{\rho} ; r)$ yields eigenstates $\Phi_{n}(\vec{\rho} ; r)$ and eigenvalues $U_{n}(r)$. One of these eigenvalues corresponds to a potential $U(r)$ exhibiting the Efimov attraction at large $r$ (when $a_{A X} \gg \ell_{\mathrm{vdW}, \mathrm{Ax}}$ ). The relative wave function $F(r)$ for the two bosons is then simply given by the following Schrödinger equation:

$$
\left(-\frac{\hbar^{2}}{m_{A}} \nabla_{r}^{2}+V_{A A}(r)+U(r)-E\right) F(r)=0
$$

Note that in this Efimov-favoured limit, the two Efimov regimes are nearly the same $\left(s_{0} \approx s_{0}^{*}\right)$, and appear in the Born-Oppenheimer approximation as a single Efimov effect described by a single channel (corresponding to the potential $V_{A A}+U$ ) and a single threebody parameter approximating the two three-body parameters. This three-body parameter is then determined by the form of the potential $V_{A A}(r)+U(r)$. According to reference [233], $U(r)$ turns out to be negligible in the short-range region, and therefore the shortrange phase of $F(r)$ is set by the van der Waals potential $V_{A A}$ only. As is known from the theory of van der Waals potentials [291, 292], that phase depends only on the van der Waals tail of this potential and its scattering length $a_{A A}$. As a result, the three-body parameter depends only on the van der Waals length $\ell_{\mathrm{vdW}, \mathrm{AA}}$ and the scattering length $a_{A A}$. If $\ell_{\mathrm{vdW}, \mathrm{Ax}}>\ell_{\mathrm{vdW}, \mathrm{AA}}$, it may also depend on $\ell_{\mathrm{vdW}, \mathrm{Ax}}$ since the potential $V_{A A}+U$ may depend on the van der Waals tail of $V_{A X}$ at intermediate distances. The conclusion is therefore the same as for the Efimov-unfavoured limit: the threebody parameters depend only on the van der Waals lengths $\ell_{\mathrm{vdW}, \mathrm{AA}}$ and $\ell_{\mathrm{vdW}, \mathrm{Ax}}$, and the scattering length $a_{A A}$. However, the origin of this universality is different from that of the Efimov-unfavoured limit and the case of three identical bosons. Instead of a three-body repulsion, it is the van der Waals potential $V_{A A}$ between the two bosons that sets the three-body parameters, the same way it sets the scattering length between the two bosons.

The authors of reference [233] have checked that this conclusion, obtained from the Born-Oppenheimer picture, is validated by exact calculations using the fullycoupled hyper-radial equations for mass ratios ranging from 14 to 29. For these large mass ratios, they obtained a very good agreement between the BornOppenheimer and exact calculations for the wave functions and energies, and demonstrated that the results are nearly insensitive to the number of bound states in the two-body Lennard-Jones potentials $V_{A A}$ and $V_{A X}$.

\subsection{Other types of short-range interactions}

The discovery of the van der Waals universality for three particles rekindles the question of which con- 
ditions lead to a simple determination of the threebody parameter. The van der Waals universality for two particles is a particular case of the universality of power-law tail potentials, which decay as $1 / r^{n}$, with $n>3$. One can therefore expect that a similar universality exists for three particles interacting via powerlaw potentials. As for other finite-range interactions, reference [38] indicates that van der Waals universality likely extends to any finite-range interaction that sufficiently suppresses the two-body probability at short distance. Their conclusion is drawn from the similitude between the three-body parameter for deep PöschlTeller potentials [293, 294] and that of deep van der Waals potentials, once both are expressed in units of effective range. The relevant length scale for the threebody parameter would therefore be the effective range $r_{e}$, which in the case of van der Waals interactions is simply related to the van der Waals length [292, 278].

\subsubsection{Two-body correlation and effective range}

Extending the two-body analysis of section 11.2.1 to arbitrary potentials qualitatively corroborates this point: if the interaction suppresses the two-body probability within some range $r_{0}$, the restriction of three-body configurations at small sizes imposes the three-body system to deform, and the kinetic energy of that deformation creates a three-body repulsive barrier at a hyper-radius comparable with the two-body suppression range $r_{0}$. The effective range $r_{e}$ provides a good estimate of the suppression range. At unitarity, the effective range $r_{e}$ is defined by [70]

$$
\frac{1}{2} r_{e}=\int_{0}^{\infty} d r\left[\bar{\varphi}(r)^{2}-\varphi(r)^{2}\right]
$$

where $\bar{\varphi}(r)=1$ is the asymptotic form of $\varphi(r)$ that is a solution of the free two-body problem. By construction, if the amplitude of $\varphi$ is suppressed within some range $r_{0}$ with respect to that of $\bar{\varphi}$, then the effective range is positive, and $\frac{1}{2} r_{e}$ is a good estimate of $r_{0}$. Therefore the location of the three-body repulsive barrier, and thus the three-body parameter, should be given by the effective range.

Of course, the precise value of the three-body parameter should depend upon the precise location and shape of the repulsive barrier. These in turn should depend upon the precise shape of the two-body wave function $\varphi(r)$. For interactions with a van der Waals tail, we know that $\varphi(r)$ has a universal form in the region of suppression given by equation (11.14), and thus the three-body parameter is universally related to $\ell_{\mathrm{vdw}}$. More generally, for interactions with a power-law tail decaying as $-C_{n} / r^{n}(n>3)$, the two-body wave function at zero energy has the following universal form at unitarity:

$$
\varphi(r) \underset{r \gtrsim \ell_{n}}{=} \Gamma\left(\frac{n-1}{n-2}\right) \sqrt{x} J_{\frac{1}{n-2}}\left(2 x^{-(n-2) / 2}\right)
$$

where $x=r / \ell_{n}$, and $\ell_{n}=\left[\frac{1}{n-2} \frac{\sqrt{m C_{n}}}{\hbar}\right]^{\frac{2}{n-2}}$. With this notation, $\ell_{6}=\ell_{\mathrm{vdw}}$. For each power $n$, there should thus be a universal relation between the three-body parameter and $\ell_{n}$. In contrast, for interactions decaying faster than power laws, such as exponentially decaying potentials, there is no such universality of the two-body wave function. Only when such potentials are very deep does the two-body wave function show a very abrupt depletion of probability that approaches a step function [82]:

$$
\varphi(r)= \begin{cases}0 & \text { for } r<\frac{1}{2} r_{e} \\ 1 & \text { for } r \geq \frac{1}{2} r_{e}\end{cases}
$$

The work of reference [82] investigates these ideas quantitatively using the separable potential approximation described in section 11.2.1. The authors checked that for various finite-range interaction potentials near unitarity (Gaussian, exponential [294], Pöschl-Teller [293, 294], Yukawa [295, 294], Morse [296, 294], 6-12 Lennard-Jones [287]), the separable potential (11.15) built with the corresponding zero-energy two-body wave function $\varphi$ does reproduce within a few percent the three-body parameter extracted from exact calculations for three identical bosons [279]. This confirms that, for pairwise potentials inducing a pronounced suppression of two-body probability, the three-body parameter is essentially governed by the zero-energy two-body wave function.

As a result, it follows that the three-body parameter for such interactions roughly scales with the effective range. The numerical values of the three-body parameters for all the potentials considered in [82], when expressed in units of the effective range of the potential, differ by just a factor of two. Namely,

$$
\kappa_{*}^{(0)} \in[0.2,0.4] \times\left(\frac{1}{2} r_{e}\right)^{-1}
$$

Although this does not constitute a universal result, it is a significant reduction of variance with respect to the Efimov $\log$-period $\left[1, e^{\pi / s_{0}} \approx 22.7\right]$ allowed by the zerorange theory. This fact may also be seen in the context of functional renormalisation group (FRG) [297]. In this framework, one applies a regulator that cuts off momenta smaller than $k$, and looks at the flow of quantities, such as the effective three-body coupling constant, as a function of $k$. The occurrence of the Efimov effect at zero energy and the unitary limit implies a limit cycle, i.e. a flow that is log-periodic in $k$, instead of a fixed-point limit. This can be intuitively understood by considering the log-periodic three-body wavefunction at zero energy in equation (4.35) confined in a box of size $1 / k$. The phase of the log-periodic oscillations is related to the three-body parameter. The authors of reference [297] checked that plotting the flow as a function of $k r_{e}$ for different separable potentials (similar to those of reference [82]) results in phase differences that are small compared to $2 \pi$. This confirms 
that the three-body parameter for these potentials is roughly determined by the effective range, in accordance with equation (11.23).

\subsubsection{Deep-potential limits}

The exact calculations of reference [279] only feature potentials near unitarity with at most one $s$-wave twobody bound state. The authors of reference [82] then extended their separable potential calculations to the case of deeper potentials near unitarity. Within the separable potential approximation, this amounts to constructing the separable potential from a two-body wave function with a larger number of nodes corresponding to the number of $s$-wave bound states in the original potential.

\section{Power-law potentials}

For potentials with a power-law tail $r^{-n}$, because of the universality of the two-body wave function given in equation (11.21), changing the depth of the potential results in little change in the three-body parameter. In the limit of deep potentials, the authors numerically found the following three-body parameters:

$$
\begin{aligned}
\kappa_{*} & =0.364(1) /\left(\frac{1}{2} r_{e}\right) \text { for } n=4 \\
\kappa_{*} & =0.2614(1) /\left(\frac{1}{2} r_{e}\right) \text { for } n=6
\end{aligned}
$$

Here, the effective range $r_{e}$ is given by

$$
\begin{aligned}
\frac{1}{2} r_{e} & \approx \frac{\pi}{\sin \left(\frac{\pi}{n-2}\right)} \frac{2^{\frac{2}{n-2}}}{n-2} \frac{\Gamma\left(\frac{n / 2+1}{n-2}\right)}{\Gamma\left(\frac{n / 2}{n-2}\right) \Gamma\left(\frac{n+1}{n-2}\right)} \ell_{n} \\
& \sim\left(\frac{n-2}{n-1}\right)^{\frac{1}{n-2}} \frac{2(n-2)^{2}}{(n-3)(2 n-5)} \ell_{n}
\end{aligned}
$$

which can be obtained from equations (11.20) and (11.21). Note that for $n=4$ and $n=6$, equation (11.26) reduces to $\frac{1}{2} r_{e} / \ell_{4}=\frac{2 \pi}{3} \simeq 2.0944$ and $\frac{1}{2} r_{e} / \ell_{6}=\frac{16 \Gamma\left(\frac{5}{4}\right)^{2}}{3 \pi} \simeq 1.39473$, respectively, and $\frac{1}{2} r_{e} \approx$ $\ell_{n}$ for large $n$.

\section{Faster-than-power-law potentials}

For potentials decaying faster than a power law, as the depth of the potential is increased, the two-body wave function slowly converges to the step function given by equation (11.22). As a result, the three-body parameter slowly converges to a universal limit, which reference [82] found to be numerically:

$$
\kappa_{*}=0.2190(1) /\left(\frac{1}{2} r_{e}\right) .
$$

\subsubsection{Classes of universality}

The authors of reference [82] concluded that there are two classes of universality for the three-body parameter: the class of potentials decaying as power law, which exhibit a robust universality, and the class of potentials decaying faster than power laws, which give a universal parameter only in the limit of very deep potentials. The deep-potential limits are continuously connected, because the two-body wave function (11.21) for power-law potentials also tends to the step function (11.22) for very large $n$. Hence, as the power $n$ is continuously increased, the universal value of the three-body parameter continuously decreases from equations (11.24-11.25) to equation (11.28), but requires deeper and deeper potentials to be reached.

It is important to note that these results are obtained within the separable potential approximation; they need to be confirmed and refined by exact calculations.

Finally, let us remark that the square-well potential

$$
V(r)=\left\{\begin{array}{ll}
-V_{0} & \text { for } r<r_{0} \\
0 & \text { for } r \geq r_{0}
\end{array},\right.
$$

which is often used in model calculations, stands out as a particular case. Its two-body wave function at unitarity does not show a suppression of probability at short distance, even when the depth $V_{0}$ is increased, and does not converge to equation (11.22). Therefore, it is not expected to exhibit the deformation-induced three-body repulsion, nor the universal three-body parameter of equation (11.28). The problem stems from the absence of tail: there is only an abrupt variation of the potential that precludes any acceleration, even classically. If the abrupt variation is smoothed a bit, the three-body parameter should eventually reach the universal value of equation (11.28) in the limit of deep wells.

\subsection{Coupled-channel interactions}

The preceding discussions only considered interactions described by a single potential. In many physical systems with resonant interactions, the reality is more complex as the interaction involves the coupling of potentials from different channels, corresponding to different internal states of the colliding particles. For instance, as explained in section 4.7, the two-body interaction between ultra-cold atoms is made resonant by using Feshbach resonances [28, 126], which result from the coupling between two or more hyperfine channels. In such situations, the value of three-body parameter may not follow the results presented in the previous sections, and depends on the characteristics of the coupled channels. Here, we consider the case of isolated Feshbach resonances.

\subsubsection{Feshbach resonances}

In the neighbourhood of a Feshbach resonance, two scattering particles at relative energy $E=\frac{\hbar^{2} k^{2}}{m}$ approach each other in some entrance channel corresponding to their internal states, but during their colli- 
sion can couple to a bound state of energy $E_{c}$ in a closed channel (i.e. whose potential dissociates above the energy $E$ ) corresponding to different internal states. The $s$-wave scattering phase shift is then the sum of two contributions:

$$
\delta_{0}(k)=\delta_{0, \mathrm{bg}}(k)+\delta_{0, \mathrm{res}}(k)
$$

The first contribution is the background phase shift $\delta_{0, \mathrm{bg}}$ corresponding to the entrance channel, and the second contribution is a resonant phase shift $\delta_{0, \text { res }}$ induced by the coupling to the bound state. It has a Breit-Wigner form [43, 126]:

$$
\tan \delta_{0, \text { res }}(k)=-\frac{\frac{1}{2} \Gamma(k)}{E-E_{C}-\Delta(k)},
$$

where $\Gamma$ and $\Delta$ are the width and shift of the resonance.

At small scattering energy, $\Gamma(k) \approx \alpha k$, and thus $\delta_{0 \text {,res }}$ can be expanded as:

$$
\frac{k}{\tan \delta_{0, \mathrm{res}}(k)}=-\frac{1}{a_{\mathrm{res}}}-R_{*} k^{2}+o\left(k^{2}\right)
$$

where $a_{\text {res }}=-\frac{\frac{1}{2} \alpha}{E_{c}+\Delta(0)}$ and $R_{*}=\frac{2 \hbar^{2}}{m \alpha}$. It follows that the scattering length $a$ is

$$
a=a_{\mathrm{bg}}+a_{\mathrm{res}},
$$

where $a_{b g}=-\lim _{k \rightarrow 0} \tan \delta_{0, b g}(k) / k$ is the background scattering length. The resonance condition is met when the energy of the bound state $E_{c}$ is tuned to compensate the shift $\Delta(0)$, making $a_{\text {res }}$, and thus $a$, divergent.

The strength of the resonance is characterised by the length $R_{*}$. When $R_{*}$ is much smaller than the range $b$ of the interaction (the van der Waals length $\ell_{\mathrm{vdw}}$ in the case of atoms), the resonance is strong and dominated by the entrance channel; the particles are most likely to be found in the entrance channel. Such entrancechannel dominated resonances usually (although not necessarily) occur over a broad range of the tuning parameter (such as an applied magnetic field), and thus are also called broad resonances. On the other hand, when $R_{*}$ is much larger than the range $b$, the resonance is weak and dominated by the closed channel. Such resonances are usually (although not necessarily) observed as narrow resonances. The strength of a resonance is thus conveniently characterised by the dimensionless ratio $s_{\text {res }}=b / R_{*}$.

\subsubsection{Broad resonances}

Broad resonances are dominated by their entrance channel and can be effectively described by a single potential [126]. The results of sections 11.2 and 11.3 based on single interaction potentials can therefore apply to the case of such resonances. In particular, for broad atomic Feshbach resonances, the van der Waals tail of the open-channel potential is the main feature that determines the three-body parameter.

\subsubsection{Narrow resonances}

In 2004, Dmitry Petrov [77] investigated the physics of three bosons near a narrow Feshbach resonance. For narrow resonances, $R_{*}$ is much larger than the range $b$ of inter-particle forces, which induces the strong energy dependence of equation (11.32). Close to the resonance condition, one can neglect the background contribution $\delta_{s, \mathrm{bg}}$, and comparing equation (11.32) with equation (4.41), we see that such resonant interactions have a large and negative effective range

$$
r_{e} \simeq-2 R_{*} \ll-b
$$

This confers to these systems some sort of long-range property, which has the effect of providing a three-body boundary condition for the Efimov-attracted particles at distances on the order of $R_{*}$, at much larger distance than the range of inter-particle forces.

Formally though, these systems can still be treated in the zero-range theory, on the basis of equation (11.32), implying that the scattering length $a$ in equations (4.6) or (4.7) is to be replaced by the energy-dependent scattering length $a(k)$ given by

$$
\frac{1}{a(k)}=\frac{1}{a}+R_{*} k^{2},
$$

where $k$ is the relative wave number between two particles. The work of reference [77] shows that unlike the original zero-range theory, the resulting three-body equations are well-behaved. Indeed, the presence of the term $R_{*} k^{2}$ in equation (11.35) turns the Efimov attraction (4.33) at short hyper-radius $R$ into a Coulombtype attraction $-1 /\left(R_{*} R\right)$, which does not necessitate the introduction of a three-body parameter. Another way to put it is that the three-body phase is set by $R_{*}$. Namely, the three-body phase as measured by $\kappa_{*}, a_{*}, a_{+}$was numerically found to be (up to a factor $\left.e^{n \pi / s_{0}}\right)[77,4]$ :

$$
\begin{aligned}
\kappa_{*} & \simeq 0.11 / R_{*} \simeq-0.22 / r_{e} \\
a_{+} & \simeq 2.9 R_{*} \simeq-1.45 r_{e} \\
a_{*} & \simeq 0.64 R_{*} \simeq-0.32 r_{e}
\end{aligned}
$$

which predicts accurately the quantities $a_{+}^{(i)} \approx$ $a_{+}\left(e^{\pi /\left|s_{0}\right|}\right)^{i}$ and $a_{*}^{(i)} \approx a_{*}\left(e^{\pi /\left|s_{0}\right|}\right)^{i}$, except for $i=0$, for which $a_{+}^{(0)} \simeq 3.3 R_{*}$ and $a_{*}^{(0)} \simeq 0.45 R_{*}$.

In 2008, Alexander Gogolin and co-workers [277] found an analytical solution to the three-boson problem near a narrow Feshbach resonance. Instead of the single-channel zero-range model based on equation (11.35), they used a two-channel model with no interaction in the entrance channel and a zero-range coupling between the entrance and closed channel. Integrating the corresponding Schrödinger equation, they showed that the resulting integral equation can be mapped to a single-particle Schrödinger-like equation. 
From this equation, the three-body parameter can be expressed analytically, leading to an accurate value:

$$
\begin{aligned}
& \kappa_{*} \simeq 0.11691 / R_{*} \simeq-0.23381 / r_{e} \\
& a_{-} \simeq-12.895 R_{*} \simeq 6.448 r_{e}
\end{aligned}
$$

It is from these calculations that the universal relation between $a_{-}$and $\kappa_{*}$ given in Eq. (10.1) was calculated accurately. For the ground-state trimer, the theory gives [298]

$$
\begin{aligned}
& a_{-}^{(0)} \simeq-10.90216 R_{*} \\
& a_{*}^{(0)} \simeq 0.458398 R_{*} .
\end{aligned}
$$

This calculation was generalised to the case of $2+1$ fermions [299] and to the case of $2+1$ bosons [300]. The authors have found that the three-body parameter in these systems is universally described by the effective range and the mass ratio. In the limit of large mass imbalance, in particular, Efimov trimers' energies at unitarity are analytically found as

$\left|E^{(n)}\right|=\frac{\hbar^{2}}{2 \mu R_{*}^{2}} e^{-\frac{2 \pi}{\left|s_{0}\right|}(1+n)} \times \begin{cases}4 e^{-\pi / 2} & (2 \text { light bosons }+1 \text { heavy particle }) \\ 4 e^{2 J_{0}} & (2 \text { heavy bosons }+1 \text { light particle }) \\ 4 e^{2 J_{0}} & (2 \text { heavy fermions }+1 \text { light particle })\end{cases}$

where $n=0,1,2 \ldots, J_{0}=0.505560 \ldots$, and $s_{0}$ is the imaginary solution of Eq. (6.8). The energies of the 2 heavy bosons +1 light particle system and 2 heavy fermions +1 light particle system converge to the same values since the repulsion originating from the antisymmetrisation becomes negligible in the large mass imbalance limit.

\subsubsection{Intermediate resonances}

It is remarkable that both the limit of very broad resonances and that of very narrow resonances lead to a universal three-body parameter in terms of the effective range of the interaction. In the case of broad resonances, the interaction can be described by a single potential, which lead to universal three-body parameters such as (11.24) or (11.25). In the case of narrow resonances, the large and negative effective range leads to a universal three-body parameter given by equation (11.39). An intriguing question is how these two limits are connected for intermediate resonances which are neither very broad nor very narrow. In particular, one may wonder whether it can still be universally expressed in terms of the effective range, as the two limits suggest.

The work of Richard Schmidt and co-workers [301] investigates this point using a separable potential model to describe the coupled channels. This model treats the coupling between the entrance and closed channels, but does not include any interaction in the entrance channel. As a result, it does not reproduce the deformation and three-body repulsion discussed in section 11.2.1 that lead to the universal three-body parameter for broad resonances. The authors therefore adjusted the arbitrary form of of their inter-channel coupling (an exponential function) so that the three-body parameter in the limit of a broad resonance $\left(s_{\text {res }} \gg 1\right)$ coincides with the known value for van der Waals interactions, given by equation (11.6). They could then calculate the three-body parameter as the strength of the resonance $s_{\text {res }}$ is decreased, using functional renormalisation group techniques. In the limit of narrow resonances $\left(s_{\text {res }} \ll 1\right)$, their calculation give $a_{-} \simeq-12.9 R_{*}$ and $\kappa_{*} R_{*} \simeq 0.117$, reproducing the results (11.3911.40). For intermediate resonances $\left(s_{\text {res }} \sim 1\right)$, they obtain a smooth and continuous crossover connecting the two limits ${ }^{18}$. This is in contrast with the effective range, which changes sign as the strength of the resonance reduces: for broad resonances, the effective range is positive and on the order of the true range $b$ of the interaction (see equation (11.26)) and for narrow resonance, the effective range is negative and given by the length $R_{*}$ (see equation (11.34)). This indicates that in this crossover region of intermediate resonances, the three-body parameter is not simply given by the effective range. This is to be expected because the universal mechanisms relating the three-body parameter to the effective range are different for the two limits: in the case of broad resonances, universality stems from the two-body short-range correlation given by equation (11.14), which is an off-the-energy-shell property, whereas in the case of narrow resonance, universality stems from the energy-dependence of the scattering phase shift (11.32), which is an on-the-energy-shell property.

The work of Yujun Wang and Paul S. Julienne [302] presents the most complete model so far. The twobody interaction is described by a set of two or three channels corresponding to the spin states involved in the Feshbach resonance, and the potential in each channel is modelled by 6-12 Lennard-Jones potential. By incorporating both the resonance and van der Waals physics, this kind of models is known to describe the two-body physics very accurately over an energy range comparable with the van der Waals energy $E_{\mathrm{vdW}}$, in the same spirit as multi-channel quantum-defect theory $[303,304]$. The authors of reference [302] argue that their model should equally provide an accurate description of the three-body physics, in particular the three-body parameter. Indeed, the Feshbach resonance is decribed properly, both in the limit of narrow resonances and the limit of broad resonances where the van der Waals tail of the potential determines the threebody parameter. Moreover, the Lennard-Jones potentials support more than one bound state, allowing the

\footnotetext{
${ }^{18}$ An alternative crossover was proposed in reference [288], but the model used in that work appears to rely on some invalid assumptions, such as the universal three-body parameter being set by the repulsive core of the two-body interaction potential, which is not the case, as noted in section 11.2.1.
} 
description of recombination and relaxation processes to these bound states. Solving the three-body problem numerically with such models, the authors of reference [302] could indeed reproduce the experimentally observed loss by three-body recombination or atomdimer relaxation around several resonances: a broad resonance in caesium-133 $\left(s_{\text {res }} \approx 200\right)$, a double resonance in caesium-133 $\left(s_{\text {res }} \approx 200\right.$ and $\left.s_{\text {res }} \approx 200\right)$, and a broad resonance in rubidium-85 $\left(s_{\text {res }} \approx 200\right)$. Not only could they reproduce the location of Efimov peaks giving the three-body parameter, but also the loss rate for scattering lengths outside the window of zero-range universality. This remarkable agreement is a further evidence of the three-body van der Waals universality.

The experimental Efimov features considered in reference [302] are driven by broad resonances, and therefore satisfy the van der Waals universal value (11.7) of the three-body parameter. In principle, the model could also be used to investigate the case of intermediate and narrow resonances, although this was not detailed in reference [302]. The authors point out that for these resonances the results should depend not only on the van der Waals length $\ell_{\mathrm{vdw}}$ and the resonance strength $s_{\text {res }}$ but also on other parameters such as the background scattering length $a_{\mathrm{bg}}$ (a fact missing by construction in the work of Richard Schmidt and coworkers [301]). Even for the broad resonances, their calculations indicate that while $a_{-}^{(0)}$ remains largely insensitive to $a_{\mathrm{bg}}$, the value of $a_{+}^{(0)}$ may depends on $a_{\mathrm{bg}}$. A full mapping of the parameter space for Feshbach resonances remains to be done.

\subsubsection{Experimental observations}

Broad resonances In ultra-cold atom experiments, it is usually easier to deal with open-channel dominated resonances because they correspond to broad resonances in terms of the applied magnetic field. Most of the measurements reported in Fig. 11.2 are obtained from relatively broad resonances. As mentioned in section 11.4.2, broad resonances are expected to be well described by single-channel two-body potentials. Indeed, the theoretical results of section 11.2 for singlechannel van der Waals potentials agree with the observations within $20 \%$. This agreement has confirmed the van der Waals universality of the three-body parameter for these resonances.

However, reducing the tolerance reveals some discrepancies that seem significant. First of all, the theoretical calculation of $a_{-}^{(0)} / \ell_{\mathrm{vdw}}$ for single-channel deep van der Waals potentials gives a value close to -11 , see equations (11.7) and (11.17), whereas most experimental values are above -9 . Another issue pointed out in reference [248] is that the presumably most precise experimental determinations of the three-body parameter, based on first-excited-state resonances, give the values (11.3) and (11.4) that differ significantly by $20 \%$. In the absence of systematic errors, one has to conclude that while van der Waals physics is the main ingredient determining the three-body parameter, it is not the only one. Possible candidates are the deviation from the van der Waals tail $\left(C_{8}\right.$ coefficient) in the singlechannel potential and coupled-channel effects. According to the calculations discussed in section 11.4.4, the coupled-channel effects tend to increase the value of $a_{-}$, which may make it closer to the observed values. Yet, more work is needed to refine our understanding. From the current theoretical and experimental results, one may only say that the value of $a_{-}$for the broad atomic resonances is about $-9 \ell_{\mathrm{vdw}}$, with an uncertainty of $20 \%$.

Intermediate and narrow resonances Closed-channel dominated resonances, on the other hand, are most often narrow and necessitate a fine tuning of the magnetic field, which in turn requires a high stabilisation of the intensity of the current in the coil creating the magnetic field. There are therefore much less experimental observations for narrow resonances. The experimental group of Giovanni Modugno in Florence [141] have reported the measurement of the three-body parameter for seven different Feshbach resonances in potassium39. These resonances correspond to a magnetic field of $58.92,60.1,65.67$, and 471.0 gauss for atoms polarised in the hyperfine state with projection $m_{F}=0$ along the magnetic field, 33.64, 162.35, and 560.7 gauss for $m_{F}=-1$, and 402.6 gauss for $m_{F}=+1$. The strength $s_{\text {res }}$ of these resonances has been calculated to range from 0.11 to 2.8 , and the background scattering $a_{\mathrm{bg}}$ ranges from $-1.54 \mathrm{~nm}$ to $-0.95 \mathrm{~nm}$. The group found that the measured value of $a_{-}^{(0)}$ does not vary significantly from its expected value (11.2) for broad resonances. Although the resonances are not narrow enough to be fully in the regime described theoretically in section 11.4.3, one would have expected that the observations would show some indication of the crossover between the narrow and broad limits, as suggested by the models of [301, 302]. As of now, these observations remain to be interpreted theoretically. 


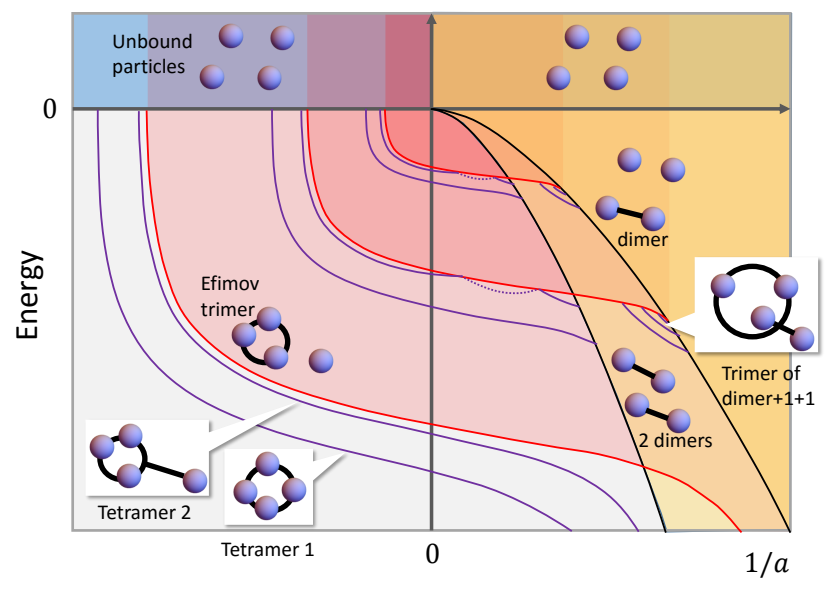

Figure 12.1: Schematic four-boson energy spectrum as a function of the inverse scattering length $1 / a$ between each pair of bosons (adapted from [33] and [306]). The black curves represent the dimer+boson+boson and dimer+dimer thresholds. The red curves represent the trimer+boson thresholds (compare with the trimer energies of figure 4.2). The solid purple curves represent the tetramer energies, and the dashed purple curves correspond to inelastic virtual tetramer states. Here, the two tetramers associated with the groundstate trimer do not follow exactly the universal pattern exhibited by the tetramers tied to excited trimers, although this depends on the microscopic model.

\section{Part V}

\section{More than three particles}

After the Efimov effect was discovered, a natural question was whether the same effect could apply to a larger number of particles. In 1973, Amado and Greenwood [305] already concluded that there is no Efimov effect for four identical bosons or more, in the sense that for $N \geq 4$, there is not an infinite number of $N$ body bound states near the appearance of an $(N-1)$ body bound state, as is the case for $N=3$. In spite of this early negative result, it was later found that not only a 4-body Efimov effect can occur for massimbalanced fermions, but a variety of universal $N$-body bound states were found in the region where the 3-body Efimov effect occurs. Here, we review the situation for bosons and mass-imbalanced Fermi mixtures.

\section{Bosons}

\subsection{Tetramers tied to Efimov trimers}

\subsubsection{Four identical bosons}

Although Amado and Greenwood [305] found from the trace of the four-body kernel of the four-boson integral equation that no four-body Efimov effect occurs, they also acknowledged that near a two-body resonance where the three-body Efimov effect occurs, there should also be four-body bound states. However, one fundamental question was whether in the limit of zero-range interactions a four-body parameter is required to set the four-body energy, just as a three-body parameter is required to set the three-body energy. Amado and Greenwood's result suggests that, in the limit $|a| \gg b$, no four-body parameter is required. As a further evidence, J. A. Tjon found by solving numerically the four-body problem for different models that the four-body energy is correlated with the three-body energy, a correlation referred to as the "Tjon line" [307, 308].

On the other hand, the works of Sadhan K. Adhikari, Tobias Frederico, I. D. Goldman, and Yamashita [309] on the perturbative renormalisation of the few-body problem with delta function potentials, and subsequent works [310, 311], advocated the introduction of an $(N+1)$-parameter for each particle added to the $N$-body system. This question was also addressed by Hans-Werner Hammer, Lucas Platter, and Ulf-G. Meißner [312][34], by solving the Yakubovsky equations [313] (a generalisation to four bodies of the Faddeev equations) with a Gaussian separable two-body and three-body potentials. The strengths of these potentials are adjusted (renormalised) such that the twobody and three-body energies are independent of the Gaussian cutoff $\Lambda$ of the potentials. The authors found that there are two tetramer states below the groundstate trimer and that their energy is relatively independent of the cutoff $\Lambda$, suggesting that no four-body renormalisation, and thus no four-body parameter is required. This led to some controversy on the necessity of a four-body parameter.

The work of Hans-Werner Hammer and Lucas Platter [34] also suggested that the presence of two tetramer states below the ground-state trimer is a universal feature that also occurs below each of the excited trimer states. This point was confirmed by Javier von Stecher, José P. D'Incao and Chris H. Greene in their study [35] where they solved the four-boson problem in hyperspherical coordinates. The use of four-body hyperradial potentials reveals the presence of a well below each trimer-boson scattering threshold, following the Efimov geometric scaling of these thresholds. Each potential well can support two bound states, one of which being just below the trimer-boson threshold, that is to say a trimer weakly bound to a boson. The existence of these states was confirmed by solving the coupled hyper-radial equations using the correlated Gaussian basis set expansion. This infinite set of tetramer states have been referred to as "universal tetramers tied to (or associated with) Efimov trimers", to avoid the designa- 
tion "Efimov tetramers" which would suggest a fourbody Efimov effect ruled out in reference [305].

The work of von Stecher and co-workers also proposed a solution to the controversy on the necessity of a four-body parameter. Indeed, the location of the well in the four-body hyper-radial potential moves to a larger hyper-radius by a factor of 22.7 at each new trimer-boson threshold, making the corresponding tetramer states essentially insensitive to any short-range four-body force, as advocated in $[312,34]$. On the other hand, the well associated with the ground-state trimer-boson threshold is located at shorter hyper-radii comparable with the range of interaction, thus making the corresponding tetramers sensitive to a short-range four-body force. This sensitivity makes to some extent the ground-state tetramer energies independent of the trimer energy, thus requiring in zero-range models the introduction of the four-body parameter advocated in [310, 311].

The precise four-body spectrum for excited states was calculated by Arnoldas Deltuva in a series of papers [314, 315, 316, 317, 318, 319, 320, 321, 322, 306]. This calculation had been challenging because the excited tetramers are resonant states embedded in trimer-boson continua. Deltuva obtained the binding energies $B_{n, i}$ and widths $\Gamma_{n, i} / 2$ of these states by calculating four-body scattering properties using the Alt-Grassberger-Sandhas (AGS) equations [323, 324]. Here, $n$ refers to the $n$-th trimer of binding energy $b_{n}$, which the two tetramers $i=1,2$ are associated with. At the unitary limit, and for large $n$, the following universal relations were found:

$$
\begin{aligned}
& B_{1}+i \Gamma_{1} / 2=(4.610(1)+i 0.01483(1)) b_{n} \\
& B_{2}+i \Gamma_{2} / 2=(1.00227(1)+i 0.000238(1)) b_{n}
\end{aligned}
$$

The whole four-body spectrum is shown in figure 12.1. Generally speaking, each pair of tetramers follows the trimer energy. The tetramer states thus form a superposition of two geometrical series with the Efimov scaling ratio $e^{\pi / s_{0}} \approx 22.7$. The two tetramers appear from the four-body threshold before the appearance of the associated trimer and dissociate in the dimer-dimer scattering threshold. However, Deltuva found that in the case of the tetramer resonances, the most weakly bound state first dissociates in the trimerboson threshold, surviving as a inelastic virtual state, before reappearing from that threshold and eventually dissociating in the dimer-dimer threshold. There is an additional family of tetramer states which appear near the crossing of a trimer and the dimer-boson threshold. These tetramer states are simply a consequence of the Efimov effect occurring for the system of dimer and two bosons, since the scattering length between the dimer and boson is resonant near this crossing. These particular features may not occur for the ground state tetramers, depending on the details of the short-range interaction.

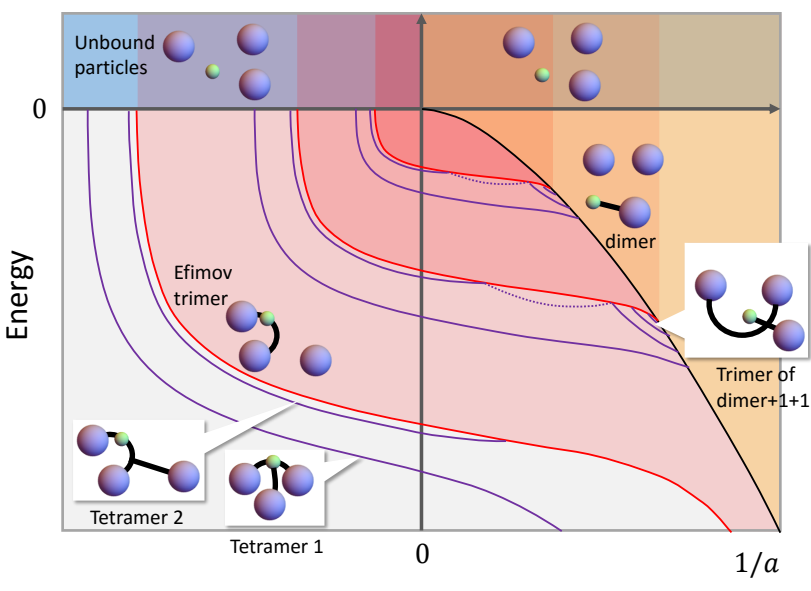

Figure 12.2: Schematic energy spectrum of three heavy bosons and one light particle as a function of the inverse scattering length $1 / a$ between the heavy boson and light particle (adapted from Refs. [325, 326]). The same conventions as those of figure 12.1 are used. It is assumed that the mass ratio between heavy and light is larger than $\sim 13$. For mass ratios smaller than $\sim 13$, the second tetramer state would disappear at a negative value of the scattering length, instead of positive. The virtual states indicated by the dashed curves are a conjecture made by analogy with figure 12.1.

\subsubsection{3 bosons +1 particle}

The four-body Efimov spectrum was investigated in the case of heavy and light bosons mixtures by Yujun Wang and co-workers [325], and subsequently by Doerte Blume and Yangqian Yan [326]. As in the case of identical bosons, there appears to be no four-body Efimov effect in these mixtures, despite earlier claims based on the Born-Oppenheimer approximation [327]. On the other hand, the works of Refs. [325, 326] show that, as in the case of identical bosons, universal tetramer states consisting of three heavy and one light bosons are tied to the heavy+heavy+light Efimov trimers (see section 6.2.1 for a discussion of these trimers). The schematic four-body spectrum is given in figure 12.2. Tetramer states tied to excited trimers were evidenced in reference [325] from the calculated four-body recombination rate, while the authors of reference [326] only calculated the tetramers tied to the ground-state trimer but conjectured that similar tetramers exist for each excited trimer. In this latter work, two tetramers were found below the groundstate Efimov trimer. They appear at negative scattering lengths, but the excited tetramer disappears in the trimer+heavy threshold for the mass ratio $\kappa \lesssim 13$ before reaching the unitary limit, while it persists to some positive scattering length for $\kappa \gtrsim 13$. On the other hand, in the work of reference [325], only one tetramer state was found at mass ratios $\kappa=30$ and 
$\kappa=50$. It is possible that the second tetramer was missed in that study because of its very weak binding energy.

In reference [326], the ground-state pentamer and hexamer were also calculated. They were found to follow the energy of the ground-state tetramer, as in the case of identical bosons.

\subsection{Universal clusters}

The previous results on universal tetramers tied to Efimov trimers naturally raise the question of the universality of larger cluster of bosonic particles. Although there is an extensive theoretical literature on bosonic clusters, motivated in particular by helium droplets, recent works have focused on the connection to Efimov physics, and in particular whether bosonic clusters with resonant interactions can be universally described by a scattering length and a three-body parameter, as in the case of universal tetramers.

This question has not been conclusively answered yet, because it requires the daunting task of calculating excited $N$-body cluster resonant states embedded in scattering continua of sub-clusters. So far, most studies [328, 329, 9, 330, 331, 332] have focused on the $N$-body bound states below the ground-state trimer, although one study [329] was able to find $N$-body resonances up to $N=6$ below the first-excited trimer. These studies give us some idea of the properties of the conjectured universal clusters associated with excited Efimov trimers.

\subsubsection{Clusters below the ground-state trimer}

The earliest attempt at calculating the $N$-body clusters tied to the ground-state Efimov trimer is the work of Javier von Stecher [328], solving by the Diffusion Monte-Carlo method a model consisting of two-body square-well interactions and three-body repulsive hardcore interactions. Von Stecher found that the $N$-boson ground-state cluster systematically appears at a weaker two-body attraction than the $N$-1-boson cluster, and remains at a lower energy. This Borromean binding property can be easily understood from the kinetic and interaction energy counting argument given in section 1 . In this study, only the ground bound state has been calculated. In a subsequent study [329] using different potential models and numerical techniques, von Stecher identified three bound states for $N=5$, and two bound states for $N=6$. These results are qualitatively summarised in figure 12.3.

In the work of Mario Gattobigio and Alejandro Kievsky [331], the same problem was investigated for systems of up to $N=6$ bosons interacting either through two-body Gaussian potentials, or two-body Pöschl-Teller potentials. In each case, the first and second $N$-body bound state were calculated. The results with Gaussian two-body potentials were confirmed by Yangqian Yan and Doerte Blume [333, 332], who also

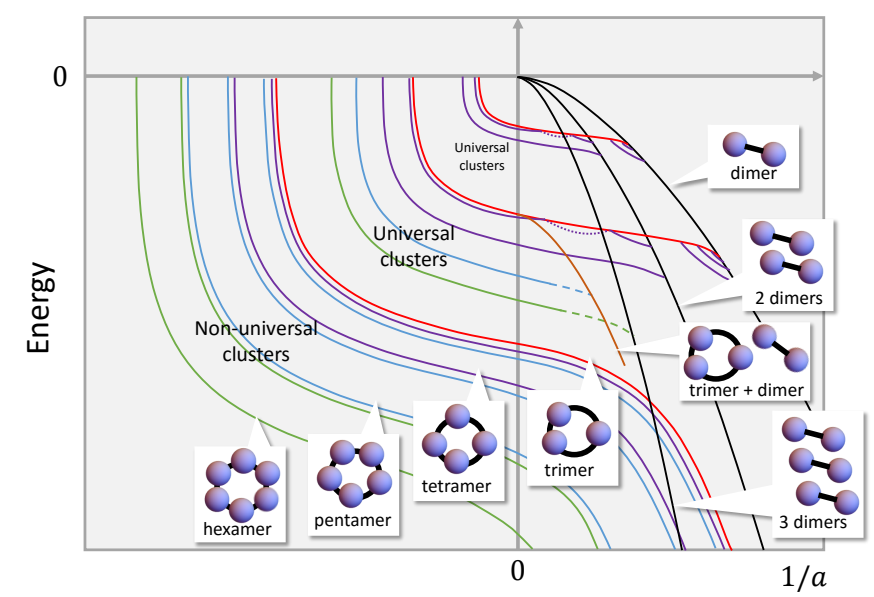

Figure 12.3: Schematic spectra of the $N$-boson clusters up to $N=6$, as a function of the inverse scattering length $1 / a$ between the bosons. This figure is based on the qualitative results of Refs. [328, 329, 331]. Trimers and tetramers correspond to the red and purple curves, as in figure 12.1, and pentamers and hexamers are shown in blue and green, respectively. Clusters below the ground-state trimer are non-universal, as they depend on the details of the interactions, whereas clusters below the excited trimers are believed to approach a universal pattern. This pattern is not well known: only a a few states have been calculated in reference [329] for negative scattering lengths. The region of positive scattering lengths offers many possible crossings and is virtually unknown.

obtained results for two-body Lennard-Jones potentials, adjusted two-body Helium-4 potentials, and twobody zero-range interactions with power-law $C_{p} R^{-p}$ three-body repulsive interactions.

Although all these results agree qualitatively with figure 12.3, they quantitatively disagree. Figure 12.4 shows the energy of the ground state cluster for some of these models. These models deviate as the number of particles $N$ is increased. This is not unexpected: as we have learnt from the preceding sections, the groundstate properties often deviate significantly from the universal pattern exhibited by excited states because the spatial extent of the ground states is comparable to the range of the interactions. Interestingly, though, Gattobigio and Kievsky showed in reference [331] that these non-universal deviations (for their own results and those of reference [328]) could be mapped back for all $N$ (up to $N=6$ ) to a single universal curve corresponding to the Efimov trimer curve given by equation (4.39), generalising the modified universal formula (4.42) for $N>3$. This observation suggests that there is a yet to be understood relationship between the non-universal ground-state clusters and the universal Efimov trimer structure. 


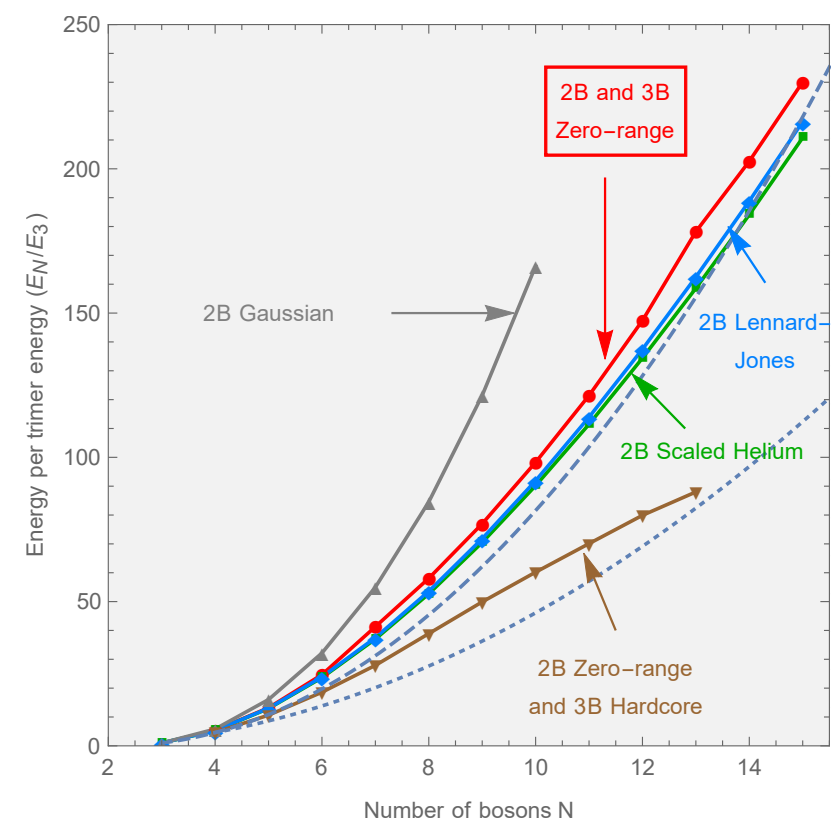

Figure 12.4: Bosons at unitarity (adapted from Refs. [328, 331, 332]): Energy of the ground-state bosonic cluster (normalised by the ground-state trimer energy) as a function of the number of bosons, for different models of interactions at unitarity $(1 / a=0)$ indicated by the arrows. The dotted and dashed curves are analytical predictions from Refs. [334] and [331], respectively.

\subsubsection{Universal $N$-body clusters}

Much less is known about the clusters tied to excited Efimov trimers, which are resonant states presumed to have a universal structure. In reference [329], Javier von Stecher could identify some of these states up to $N=6$. They are schematically shown in figure 12.3 , and qualitatively follow the pattern of the clusters associated with the ground-state Efimov trimer for negative scattering lengths. This indicates that the groundstate clusters give us some idea of the spectrum of the excited clusters. The earlier work of reference [328] attempted to make these ground-state clusters close to the universal clusters by using a model consisting of two-body square-well interactions and three-body hard core repulsive interactions. The resulting ground-state pentamer and hexamer were indeed found to agree relatively well with the excited pentamer and hexamer resonances found in reference [329], much more so than the ground-state clusters of other models. Nevertheless, it is unclear how much universal this model is, especially for large $N$.

To improve this situation, Yan and Blume considered an alternative model in an attempt to approach a purely zero-range model, which supposedly yields universal results for both the excited and ground clusters (in fact, a purely zero-range model has no ground state). Although two-body zero-range interactions can be implemented in their numerical method (based on the path integral Monte Carlo method), three-body zero-range interactions cannot be treated. The authors thus resorted to a three-body repulsive interaction of the form $C_{p} R^{-p}$, where $R$ is the three-body hyperradius - see equation (4.24), and carefully checked that when $p$ is increased, the three-body ground-state observables convincingly converge to that of the universal (zero-range) theory. It is thus plausible that the ground-state clusters of this model converge to the universal clusters for large enough $p$. Although the cluster energies are found to be rather insensitive to $p$ for small $N$, for larger $N$ their energies become strongly dependent on $p$. Nevertheless, we noticed that the dependence on $1 / p$ appears to be linear, which makes it easy to extrapolate to $p \rightarrow \infty$. In figure 12.4 , we have represented the result of this extrapolation by the red curve. At present, this curve represents the most plausible variation of the energy with $N$ for universal clusters at the unitarity limit. Interestingly, it turns out to be close to the curves obtained for ground-state clusters for Lennard-Jones and scaled helium potentials, although these curves are expected to represent a different class of universality associated with van der Waals potentials.

There remain many open questions about the universal clusters. The region of positive scattering lengths have not been addressed yet, and promises to reveal a rich pattern of clusters dissociating into subclusters. There is also an intriguing possibility that the energy of clusters of a very large number of particles, if they exist, may leave the scaling window of the trimer they are originally associated with.

\subsection{Observation with atoms}

In the theoretical study reference [35] on tetramers tied to Efimov trimers, it was realised that experimental evidence of these tetramers could be seen through the enhancement of the four-body recombination loss rate at the scattering lengths where the tetramers appear at the four-body scattering threshold, something which had in fact already been observed in experimental data from ultra-cold caesium atom experiments at the University of Innsbruck. In reference [33], the experimentalists in Innsbruck confirmed that the locations of these loss peaks at $a=0.47 a_{-}$and $a=0.84 a_{-}$ (where $a_{-}$designates the scattering length at which a trimer appears, seen experimentally by a three-body loss peak) are consistent with the expected locations $a_{- \text {Tetra1 }} \approx 0.43 a_{-}$and $a_{- \text {Tetra2 }} \approx 0.9 a_{-}$. A few years later, the same group reported the observation in the same system of a weak five-body loss peak at the scattering length where a ground-state pentamer is expected to appear [335].

Two other groups, the group of Randall G. Hulet at Rice University [140] and the group of Giovanni Modugno and Massimo Inguscio in Florence [139], also re- 
ported in 2009 the observation of the four-body loss peaks at the scattering lengths consistent with the above values, but it turned out later that their results were plagued by misassignments of the loss peaks and recalibration of the scattering length with respect to the magnetic field (see section 4.7.2), and their results were modified in 2013 [141, 142]. The former group originally found two four-body loss peaks [140], but later in reference [142], they could only observe a single peak at $a=0.37 a_{-}$which is likely to correspond to one of the four-body bound states, while the other one is not observed. The latter group originally reported the observation of a single four-body loss peak at $a=0.43 a_{-}[139]$, but after reassignment of the peaks, no four-body loss peak was found [141].

\section{Mass-Imbalanced Fermi mixtures}

Resonantly interacting four-body systems with identical fermions have also been studied recently. The first treatment of these systems was the exact calculation by Dmitry Petrov and co-workers [336, 337] of the dimerdimer scattering length for a four-body system of two identical (i.e., spin-polarised) fermions plus two other identical fermions (i.e. in a different spin state). The four-body problem for fermions, while more challenging than that for identical bosons, is still tractable analytically and numerically. Systems with identical fermions are subject to the repulsion originating from the Pauli exclusion principle and are, generally speaking, less likely to support bound states than bosonic systems. However, this repulsion can be overcome by increasing the mass imbalance between the two fermionic species, leading to the formation of a four-body bound state, as in the case of mass-imbalanced three-body system with fermions (see section 6.2.5). In this section, we review the recent theoretical progress on two classes of fourbody systems which consist of resonantly-interacting identical fermions of two species: a system of two identical (i.e. spin-polarised) fermions plus two other identical fermions in section 13.1, and a system of three identical fermions plus another distinguishable particle in section 13.2. We review and discuss prospects for systems of more than four particles in section 13.2.3.

\subsection{2 fermions +2 fermions}

The earliest works on the four-body problem in a two-component Fermi mixture were motivated by the studies of the BEC-BCS crossover [338, 339, 340, 341, 342, 343, 344, 345]. The nature and stability of the Bose-Einstein condensate phase, consisting of dimers made of different fermions, is sensitive to scattering properties of the dimers. In some works [341, 342, 343, 344, 346, 347], the dimer-dimer $s$-wave scattering length has been estimated with some approximations. Exact calculations have been performed in Refs. [336, 337, 345, 348, 349], and the dimer-dimer scattering length at equal mass has been found to be $a_{d d} / a=0.59 \ldots$ ( $a$ is the $s$-wave scattering length between two non-identical fermions), which is significantly smaller than the mean-field (i.e., the Born approximation) value $a_{d d} / a=2.0$ due to the Pauli exclusion principle. The exact calculation of the elastic dimer-dimer $s$-wave scattering length has also been extended to the mass-imbalanced case in Refs. [350, 191, 351], and the asymptotic behavior at large mass ratio $a_{d d} \approx a_{a d} / 2\left(a_{a d}\right.$ is the fermion-dimer $s$-wave scattering length) has also been found analytically [352].

For a four-body system made of 2 identical fermions +2 identical fermions, there necessarily exists a repulsion between each identical fermions due to the Pauli exclusion principle, preventing the formation of a universal four-body bound state [350, 353, 354]. Indeed, for any parity and angular momentum channel, there is no universal four-body bound state when the mass ratio is smaller than the three-body Efimov critical mass ratio $\kappa_{c}^{(3)}=13.6069657 \ldots$ [355, 353, 354]: there is neither a four-body Efimov effect at the unitary limit [355, 354, 356] nor a universal four-body bound state of Kartavtsev-Malykh character for a positive scattering length [353]. This is in marked contrast to the $3+1$ Fermi system discussed in the next section, where four-body bound states can appear below the three-body Efimov critical mass ratio.

On the other hand, if the mass ratio exceeds the three-body Efimov critical mass ratio $\kappa_{c}^{(3)}=$ $13.6069657 \ldots$, the three-body Efimov attraction arises and may bind the four particles. The resulting fourbody bound states, however, are not expected to be four-body Efimov states. Since the introduction of the three-body parameter is known to regularise the four-body problem for a bosonic four-body system $[305,312,34]$, it is expected that the four-body bound states of the $2+2$ Fermi system, if they exist, should also be characterized by the three-body parameter only, and would not require the introduction of a four-body parameter. They would therefore be tied to the three-body Efimov states, in an analogous manner to the four-body bound states of a Bose system (see section 12 and Refs. [312, 34, 35, 316, 325]), and show the discrete-scaling symmetry with the same scaling factor as the Efimov trimers.

\subsection{3 fermions +1 particle}

In contrast to the $2+2$ Fermi system, the system of 3 identical fermions of mass $M$ plus a distinguishable particle of mass $m$ exhibits richer physics. In particular, the repulsion between the identical fermions can be overcome by the attraction mediated by the other particle when the mass of the identical fermions is much larger than that of the other particle. This situation 
is similar to the $2+1$ system (see section 6.2.5). As a result, physics similar to that of the $2+1$ Fermi system has been found to occur in the $3+1$ Fermi systems, as discussed below.

\subsubsection{Four-body Efimov effect}

Yvan Castin and co-workers have studied the $3+1$ system at the unitary limit, and have found that a fourbody Efimov effect occurs, i.e. the existence of a fourbody attraction leading to an infinite number of fourbody bound states in the absence of three-body bound states. This effect occurs in the $L^{\Pi}=1^{+}$channel, where $L$ is the total angular momentum and $\Pi$ is the parity of the four-body state, and for a mass ratio $M / m$ above the critical ratio $\kappa_{c}^{(4)}=13.384 \ldots$ [111], which is below the critical mass ratio $\kappa_{c}^{(3)}=13.606 \ldots$ for the occurence of three-body Efimov effect for $2+1$ systems. The same result has also been obtained more recently by Betzalel Bazak and Dmitry Petrov [357]. Since there is no trimer at unitarity in the range of mass ratio $\kappa_{c}^{(4)}<M / m<\kappa_{c}^{(3)}$, the binding of the $3+1$ states is a genuine four-body phenomenon. These four-body Efimov states exhibit discrete scale invariance with a scaling factor $e^{\pi / s}$, where the exponent $s$ in the scaling factor is obtained by numerically solving the four-body Schrödinger equation at the unitary limit. As in the three-body Efimov effect, this leads to a transcendental equation that determines the value of $s$. It is worthwhile to note that this is the first and so far only known example of the four-body Efimov effect in any physical system: for a four-body system of bosons, the four-body Efimov effect does not occur [305], and there exists only a finite number of four-body bound states tied to each Efimov trimers $[312,34,35,316]$ - see section 12.1. It is also interesting to note that some of the four-body Efimov states are likely to persist beyond the three-body critical mass ratio $\kappa_{c}^{(3)}=13.606 \ldots$, while some others are expected to mix with the trimer+fermion scattering continua.

\subsubsection{Universal four-body bound state}

Below the four-body Efimov critical mass ratio $\kappa_{c}^{(4)}=$ $13.384 \ldots$, there is no universal four-body bound state at the unitary limit. However, at positive scattering length for a mass ratio $M / m \gtrsim 9.5$, a universal four-body bound state was found numerically by Doerte Blume in reference [353] in the same $L^{\Pi}=1^{+}$ channel as the four-body Efimov states. This fourbody bound state is universally characterised by the $s$-wave scattering length, and is independent of other short-range parameters. It can therefore be interpreted as a four-body analogue of the universal threebody bound states found by Kartavtsev and Malykh for mass ratio $M / m>8.17 \ldots$ and $M / m>12.91 \ldots$ (see section 6.2.5). Just as the Kartavtsev-Malykh three-body bound states are remnants of the threebody Efimov states appearing above the critical mass ratio $\kappa_{c}^{(3)}=13.606 \ldots$, the universal four-body bound state may be regarded as a remnant of the four-body Efimov states appearing above the critical mass ratio $\kappa_{c}^{(4)}=13.384 \ldots$ [111]. We note that the critical mass ratio for this universal four-body state has recently been refined to $M / m=8.862(1)$ [357].

Note that, as in the case of the $2+1$ Fermi system (see section 6.2.5 and Refs. [195, 355, 358, 194]), other kinds of four-body bound states may exist for the 3 +1 Fermi system at unitarity even below the critical mass ratio, but they are non-universal [355, 353, 358] in the sense that they depend on a short-range boundary condition that is not set by the scattering length but other short-range details of the interactions.

\subsubsection{Five bodies and beyond}

It is rather challenging to study the universal and Efimov states of five or more particles since it requires a highly accurate calculation of the extremely small binding energy of these clusters at the critical mass in the presence of the fermionic sign problem. Nevertheless, Betzalel Bazak and Dmitry Petrov have recently succeeded in studying the mass-imbalanced $4+1$ Fermi system by using the diffusion Monte Carlo method to solve the Skorniakov - Ter-Martirosian equation for this system [357]. They found the critical mass ratios for the Efimov pentamers and universal pentamers in the $L^{\Pi}=0^{-}$channel to be $13.279(2)$ and $9.672(6)$, respectively.

From the critical mass ratios for the $2+1,3+1$, and $4+1$ systems, it is tempting to make an extrapolation to $N+1$ system with $N \rightarrow \infty$. The extrapolation suggests that the critical mass ratios for the Efimov and universal clusters approach $\sim 13.0(1)$ and $\sim 12(2)$ in that limit. But this naive extrapolation should not be taken too seriously since it neglects shell-closureoscillation which may be significant around the $4+1$ system corresponding to the shell closure of the $p$-wave orbital in the shell model picture [357]. The authors of reference [357] expect that 5+1 Efimov hexamers or universal hexamers, if they do exist, show qualitatively different behaviours from $2+1,3+1$, and $4+1$ clusters since to form these hexamers a heavy fermion needs to occupy a higher angular momentum or radial quantum number state in the shell model picture. 


\section{Part VI}

\section{Many-body systems}

So far, our focus has been on isolated systems with a relatively small number of particles. This approach is relevant to the description of some systems such as light nuclei or isolated molecules. However, many physical systems are composed of a very large number of particles. It is thus natural to ask oneself about the implications of Efimov physics in this context. Several studies have recently started to address this question. The first studies have looked into the influence of a many-body background onto Efimov states, while more recent studies deal with the consequences of few-body Efimov physics at the many-body level.

\section{Many-body background}

The observation of Efimov physics in ultra-cold atom experiments involve the existence of triatomic molecules that can be formed from an extremely dilute cloud of atoms. Even though the density of the cloud, typically $10^{12}$ atoms per cubic centimetre, is small enough to regard the triatomic molecules as isolated, it is natural to wonder how the properties of such molecules are changed under the influence of a surrounding medium. It is particularly interesting that the medium may be composed of the same constituents as the those of the molecule, as this consideration constitutes a first step towards many-body problems. Depending on the quantum statistics of the constituents, there are thus two possible media, a Fermi sea or a Bose gas.

\subsection{Efimov states in a Fermi sea}

Let us consider an Efimov trimer containing one or several fermionic particles, and surrounded by one or several seas of fermions that are identical to those of the Efimov trimer. The presence of these many additional fermions constitutes a non-trivial many-body problem, but it can be treated in a first approximation as a static Fermi sea. In this case, the main effect of the Fermi sea is to prevent the fermions in the Efimov trimer from occupying the states already occupied by the other fermions, owing to the Pauli exclusion between identical fermions. This idea has been explored in the works of Refs. [359, 360, 361]. Generally speaking, the Pauli exclusion tends to reduce the binding of the Efimov trimer. For a large enough density of the Fermi seas, the Efimov trimer eventually disappears. The successive disappearances of the different excited Efimov states as the density is increased follow a scaling law with the universal scaling ratio $e^{\pi /\left|s_{0}\right|}[359,361]$. Interestingly, the trimers can survive at positive energy, as a "Cooper triples", in analogy with the Cooper pairs [362], a fact emphasised in reference [360].

\subsubsection{Two bosons and a fermion in a Fermi sea}

The first study on the influence of a Fermi sea on Efimov states is that of Davis James MacNeill and Fei Zhou [359]. They considered two heavy bosons of mass $M$ immerged in a sea of light fermions of mass $m$, assuming a resonant pairwise interaction between the bosons and the fermions. If the sea was composed of a single fermion, we know from section 6.2.1 that Efimov trimers exist and can be interpreted in the BornOppenheimer picture (see section 6.2.4) as the binding of the two bosons by the light fermion. The authors thus used the Born-Oppenheimer approximation, along with a semi-classical approximation, to calculate the three-body spectrum with the constraint the fermion cannot occupy states of momenta smaller than the Fermi momentum $k_{F}$ of the Fermi sea. Since this constraint breaks the translational invariance, the total momentum of the system does not simply shift the energy of the spectrum. For simplicity, the authors restricted their consideration to states of zero total momentum. For $k_{F}=0$, they retrieve the vacuum Efimov states of section 6.2.4. Increasing $k_{F}$ results in an increase of the trimer energies (a decrease of their binding energy). As a result, the infinite tower of Efimov states is gradually pushed up, until the ground state disappears in the scattering threshold. The successive disappearances of Efimov states follow a scaling law with the universal scaling ratio $e^{\pi /\left|s_{0}\right|}$. The required $k_{F}$ to observe this density dependence, however, seems to be too large for cold-atom experiments with a large mass imbalance [363].

\subsubsection{Three fermions in a Fermi sea}

A similar treatment for three kinds of fermions of equal mass was done in the works of Nicolai Gayle Nygaard and Nikolaj Thomas Zinner [361], and Patrick Niemann and Hans-Werner Hammer [360]. The work of reference [361] considered explicitly one Fermi sea associated with one of the three fermions, while the work of reference [360] considered three Fermi seas of equal Fermi momentum $k_{F}$. In both works, the problem was solved using the Skorniakov - Ter-Martirosian threebody integral equation with the Pauli exclusion constraint from the static Fermi seas. The results are qualitatively the same as those of MacNeill and Zhou [359], although the work of Niemann and Hammer [360] emphasises the existence of solutions at positive energies, which they call "Cooper triples", in analogy with the Cooper pairs.

A limitation of the previous results is that the Fermi seas are treated as static. In reality, the back action of the particles on the Fermi seas can create particlehole excitations near the surface of the Fermi sea, an effect called polarisation of the sea. The authors of reference [359] estimated that the polarisation of the sea would reduce even further the binding of the Efimov trimers. On the other hand, Charles J. M. Mathy 
and co-workers [364] found in a somewhat different system that the polarisation of the sea may on the contrary enhance the stability of trimers. This work considers the case of a trimer made of a light fermion and two heavy fermions (known as Kartavtsev-Malykh universal trimer, see section 6.2.5), surrounded by a sea of identical heavy fermions. The authors used a variational ansatz involving both the trimer and the particle-hole excitation and found that the stability of the trimer is enhanced by a moderate density of the Fermi sea.

\subsection{Efimov states in a condensate}

\subsubsection{Two impurities and a boson from a BEC}

The case of Efimov trimers in a BEC (Bose-Einstein Condensate) has been considered by Nikolaj Thomas Zinner in reference [365]. In that study, two heavy particles (impurities) resonantly interact with bosons from a surrounding condensate of bosons. At low density, it is known that one of the bosons can bind to two heavy particles to form an Efimov trimer, which can be easily interpreted in the Born-Oppenheimer approximation, as detailed in section 6.2.4. The author of reference [365] thus used the Born-Oppenheimer approximation to calculate the effective interaction between the two heavy particles at fixed separations. To treat the effect of the surrounding condensate, the author used the Bogoliubov approach $[366,367]$ to describe the bosons as a ground-state condensate with quasiparticle excitations. The author then retained only the coupling to quasi-particles, resulting in a threebody problem for two heavy impurities and a quasiparticle. Although the validity of this approximation is not clear, it leads to results that are similar to those of reference [359]. The resulting Born-Oppenheimer potential between the two heavy impurities is influenced by the medium at large separations $R$, on the order of the coherence length $\xi$ of the condensate. In this region, the bonding potential is reduced with respect to the bonding potential in the absence of condensate (see section 6.2.4 and figure 6.3), resulting in a faster decay. This in turn reduces the binding of the Efimov states, as in the case of a Fermi sea. However, in this theory, the potential appears to be not defined beyond a certain separation $R_{0} \approx 0.5 \xi$, so that these preliminary conclusions are to be confirmed by a more consistent theory.

\subsubsection{One impurity and two bosons from a BEC}

Systems of a single impurity atom resonantly interacting with a Bose condensate have recently been realised by two independent groups; one with potassium-39 atoms in two different hyperfine states by the group of Jan Arlt at Aarhus University [368], and the other one with a mixture of rubidium-87 and potassium40 by the group of Eric Cornell and Deborah Jin at
JILA [369]. Efimov states may appear in these systems since two identical bosons can bind with the impurity to form an Efimov trimer, as discussed in section 6.2.1. This possibility was explicitly taken into account for the impurity-BEC system in the work of Jesper Levinsen, Meera M. Parish, and Georg M. Bruun [370], by constructing a variational ansatz that includes up to two Bogoliubov excitations of the BEC. This allows to take into account the three-body correlations of the impurity with two bosons of the condensate. If one includes only two-body correlations, the resulting variational ground-state is a "Bose polaron", i.e. the impurity is dressed by the surrounding condensate and, as the impurity-boson interaction is increased, progressively binds with one of the bosons to form a dimer surrounded by the remaining bosons. By contrast, taking into account three-body correlations, the Bose polaron instead turns into a trimer composed of the impurity bound to two bosons and surrounded by the remaining bosons. The authors indicate that this state may be seen as the avoided crossing between the two-body correlated polaron with the vacuum ground-state Efimov trimer composed of the impurity and two bosons. In the experiments [368, 369], such an avoided crossing could not be observed since the Efimov trimers are much larger than the mean atomic distance, but it would appear if one can prepare a gas with much smaller density. Interestingly, the energy of the trimer dressed by the medium is lower than that of the vacuum trimer, showing that in this case the surrounding condensate stabilises the Efimov trimer.

Given these results, it would be tempting to think that by taking a larger number of Bogoliubov excitations, one could couple the polaron to universal clusters similar to those discussed in section 13.2, such as a tetramer formed of three bosons and the impurity. While this is certainly to be expected, the authors of reference [370] argue that the couplings to these clusters would be smaller than that to the trimer, and possibly negligible. Their argument is that the repulsive interaction between the bosons tend to reduce the coupling between the polaron and universal clusters. They checked that the coupling to the trimer is indeed reduced when the scattering length $a_{B}$ between the bosons is increased, relative to the scattering length $a_{-}$between a boson and the impurity at which the trimer appears. Since universal clusters are expected to appear at scattering lengths between a boson and the impurity that are even smaller than $\left|a_{-}\right|$, they expect that the reduction of the coupling is comparatively stronger for larger clusters. Luis Aldemar Peña Ardila and Stefano Giorgini have also arrived at a similar conclusion with a quantum Monte Carlo calculation [371].

\section{Many-body phases}




\subsection{Identical bosons}

Since it became possible to adjust the scattering length of ultra-cold atoms close to unitarity thanks to magnetic Feshbach resonances, experimentalists have hoped to realise the unitary Bose gas, an intriguing strongly-correlated system which theorists have been excited about for many years $[372,373,374,375,376$, $377,378,379]$. In particular, some theorists have wondered about the role of Efimov physics in such a gas, and how it can be described by the three-body parameter of the atoms, unlike the unitary Fermi gas which has no microscopic length scale. Unfortunately, when the scattering length is tuned to large values, ultracold bosonic gases are found to be unstable because of enhanced loss by recombination of the atoms into the many bound states that exist for atomic interactions. It is for this reason that Efimov trimers in ultracold gases have been evidenced essentially through features in these losses, rather than more direct obervations. Nevertheless a metastable unitary gas of density $\rho$ and temperature $T$ (or thermal de Broglie wavelength $\lambda=h / \sqrt{2 \pi m k_{B} T}$ ) can be prepared in the nondegenerate regime $\rho \lambda^{3} \ll 1$ [380], and it is possible to observe the gas dynamics in the degenerate regime $\rho \lambda^{3} \gtrsim 1$ [381]. Here we review some theoretical and experimental works that attempt to reveal the role of Efimov physics in an atomic Bose gas.

\subsubsection{Three-body contact in a Bose gas}

Shina Tan $[382,383,384]$ has introduced a set of universal relations for the two-component Fermi gas that relates its properties to the scattering length $a$ between two different fermions through the strength of two-particle short-range correlations, characterised by an extensive quantity called the "contact" $C_{2}$. This quantity could be directly measured through radiofrequency (rf) spectroscopy in a number of experiments with ultra-cold atomic Fermi gases. The notion of contact was generalised for bosons by several authors [385, 386, 387, 388, 389]. It appears that, in addition to the two-body contact, that gives the variation of energy $E$ of the Bose gas with respect to the scattering length $a$,

$$
\frac{d E}{d a}=\frac{\hbar^{2}}{8 \pi m a^{2}} C_{2},
$$

the occurrence of Efimov physics for bosons requires to introduce a three-body contact $C_{3}$, that gives the variation of energy with respect to the three-body parameter $\kappa_{*}$,

$$
\frac{d E}{d \kappa_{*}}=\frac{\hbar^{2} \kappa_{*}^{2}}{m} C_{3} .
$$

Contacts of the dilute Bose gas The rf response of a Bose-Einstein condensate of rubidium-85 was recently investigated experimentally at JILA [282]. The threebody contact is expected to introduce in the rf response a frequency dependence that is proportional to
$C_{3}$ and has a log-periodicity that characterises Efimov physics [388]. Although the contribution from the twobody contact was clearly seen in the experiment, no log-periodic contribution from the three-body contact could be evidenced. This was explained by subsequent calculations by D. Hudson Smith and co-workers in reference [389] which determined that for the dilute Bose gas of volume $V$ :

$$
\begin{aligned}
& C_{2} / V \approx 16 \pi^{2} a^{2} \rho^{2} \\
& C_{3} / V \approx \frac{16 \pi^{2}(4 \pi-3 \sqrt{3}) s_{0} \cosh \left(\pi s_{0}\right)}{3 \sinh ^{3}\left(\pi s_{0}\right)} a^{4} \rho^{3} .
\end{aligned}
$$

From this expression, the authors found that the value of $C_{3}$ is indeed too small to be observed in the experiment.

Contact of the unitary Bose gas In a subsequent experiment, the experimentalists at JILA were able to measure the momentum distribution in their BoseEinstein condensate of rubidium at unitarity, before the three-body losses set in and deplete the gas [381]. This distribution was found to saturate to a seemingly universal distribution. The tail of this momentum distribution is known to be related to the two-body and three-body contacts $[388,386]$. As in the rf spectroscopy case, the three-body contact introduces a contribution which is proportional to $C_{3}$ and has the Efimov log-periodicity in momentum. From dimensional analysis, D. Hudson Smith and co-workers [389] found that for the unitary Bose gas,

$$
\begin{aligned}
& C_{2} / V \approx \alpha \rho^{4 / 3} \\
& C_{3} / V \approx \beta \rho^{5 / 3}
\end{aligned}
$$

By fitting the experimental measurement of the momentum distribution, assuming that the observed variations correspond to the log-periodic prediction for the tail, the authors of reference [389] obtained $\alpha=22(1)$ and $\beta=2.1(1)$. Other theoretical works have made predictions for the value of $\alpha$, namely $\alpha=10.3$ [375], $\alpha=12$ [390], and $\alpha=9.02$ [391]. The two-body contact of the unitary Bose gas has also been calculated at finite temperature using a three-body-cutoff model [392] but the small values of the three-body contact and $\beta$ have proved difficult to obtain.

\subsubsection{The non-degenerate unitary Bose gas}

In the non-degenerate regime $\rho \lambda^{3} \ll 1$, the equation of state of the unitary Bose gas can be treated by the so-called virial expansion [48, 393]:

$$
\frac{P \lambda^{3}}{k_{B} T}=\sum_{n \geq 1} a_{n}\left(\rho \lambda^{3}\right)^{n}
$$

where $a_{1}=1, a_{2}, a_{3} \ldots$ are the virial coefficients. Because the third coefficient is related to three-body correlations, it is expected to depend on Efimov physics. 
Yvan Castin and Félix Werner have studied the virial coefficients at unitarity $1 / a=0$ [394] and found an analytical expression for the first virial coefficients in the zero-range limit:

$$
\begin{aligned}
& a_{2}=-\sqrt{2} \frac{9}{8} \\
& a_{3}=\frac{81}{8}-6 \sqrt{3} f\left(\kappa_{*} \lambda\right)
\end{aligned}
$$

where $\kappa_{*}$ is the three-body parameter defined from the ground-state trimer energy $E_{0}=-\hbar^{2} \kappa_{*}^{2} / m$ - see section 10.1, and the function $f$ admits the low- and hightemperature limits ${ }^{19}$ :

$$
f\left(\kappa_{*} \lambda\right) \simeq e^{E_{0} / k_{B} T} \quad \text { for } k_{B} T \ll E_{0}
$$

$f\left(\kappa_{*} \lambda\right) \simeq \frac{\left|s_{0}\right|}{2 \pi} \ln \left(e^{\gamma+2 \pi C /\left|s_{0}\right|} E_{0} / k_{B} T\right)$

for $k_{B} T \gg E_{0}$

where $C \simeq 0.648 \ldots$ and $\gamma \simeq 0.577 \ldots$ is Euler's constant. At high temperature, the gas therefore exhibits a dependence on temperature that has the log-periodicity associated with Efimov physics. The authors however noted that the validity of the zero-range model may break down in this limit.

Away from unitarity, the second and third virial coefficients were recently calculated numerically in reference [396], as a function of the scattering length $a$ and three-body parameter $\kappa_{*}$. These numerical results are consistent with the above analytical predictions at unitarity.

\subsubsection{The Efimov liquid phase}

The degenerate regime $\rho \lambda^{3} \gtrsim 1$ of the unitary Bose gas is more speculative since strong losses occur in experiments with atoms. Nevertheless, the recent experimental achievement of reference [381] has raised hope for the investigation of the degenerate unitary Bose gas.

The recent theoretical developments in few-body Efimov physics indicate that bosons may form universal excited $N$-body clusters governed by Efimov physics - see section 12.2. Similar clusters exist below the ground-state Efimov trimer, although they are not necessarily universal. The tendency of bosons to cluster near unitarity indicates that the system as a gas is metastable. This raises questions about the ground state of the system. The results of numerical investigations of $N$-body clusters shown in figure 12.4 suggest that the energy per particle tends to a negative constant for large $N$, although this constant depends strongly on the model. A negative constant energy per particle would imply that the system becomes a liquid for large $N$. Based on this idea, Swann Piatecki

\footnotetext{
${ }^{19}$ The calculation of the third virial coefficient has also been generalised to the case of $2+1$ fermions [395] and to the case of $2+1$ bosons [300].
}

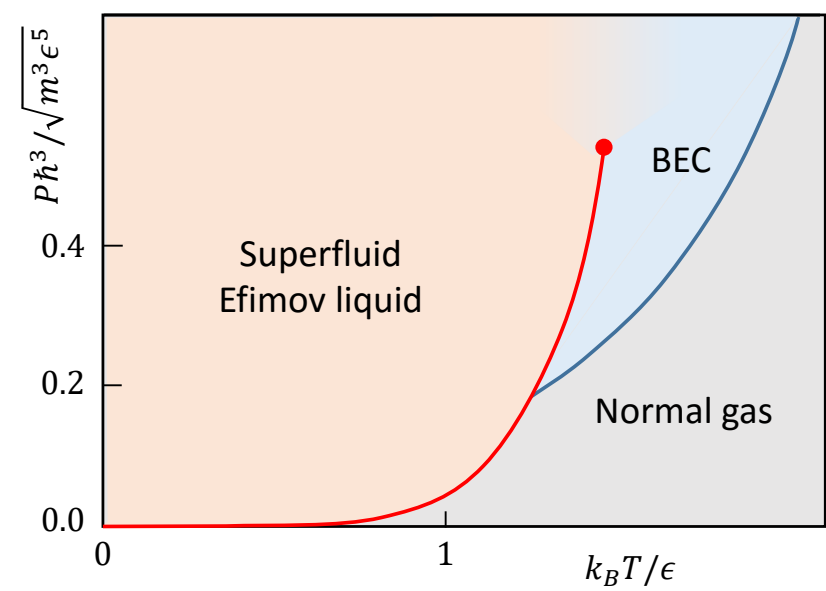

Figure 15.1: Schematic phase diagram of unitary , bosons as a function of pressure and temperature normalised by the binding energy per particle $\epsilon$ in the Efimov liquid phase (adapted from the path-integral Monte Carlo results of reference [379]). The value of $\epsilon$ is related to the energy $E_{0}$ of the ground-state Efimov trimer at unitarity, although it appears to be modeldependent (see figure 12.4). In the calculation of reference [379], $\epsilon \approx 8\left|E_{0}\right|$.

and Werner Krauth [379] investigated the possibility of a liquid phase in the unitary Bose system, using the path-integral Monte Carlo method to numerically solve the same model as that of reference [328], i.e. bosons with two-body zero-range interactions and three-body hard repulsive core. They obtain the phase diagram shown in figure 15.1 for a homogeneous system. They found indeed that for sufficiently low temperature and pressure, the system becomes a liquid, which they call the "superfluid Efimov liquid". As expected, the density of this liquid is fixed by the trimer energy, i.e. the three-body parameter. We should note that the model is quantitatively different from that of zero-range interactions or shallow van der Waals interactions, as can be seen in figure 12.4. The obtained phase boundaries are therefore likely to be neither universal nor quantitative for a realistic atomic system. Moreover, it is yet unclear how stable the Efimov liquid phase is in an realistic atomic system, which interacts through deep van der Waals potentials, since further decay would occur to more deeply bound states.

Yet, the idea of an Efimov liquid, essentially bound by the Efimov attraction is quite compelling. A particularly intriguing point is whether the discrete scale invariance still holds in this many-body system, implying that excited metastable liquid phases of densities smaller by factors of $22.7^{3} \approx 10^{4}$ could exist, at least in principle. 


\subsection{Trimer phases in Fermi mixtures}

As discussed in Section 6, the Efimov effect occurs in multi-component Fermi systems, i.e. mixtures of fermions of different kinds or with different spins that interact resonantly. Efimov trimers or related universal trimers may therefore be formed in these systems. A striking difference with bosonic systems, however, is that it is more difficult for more than three fermions to bind, due to the Pauli exclusion between identical fermions. As a result, a relatively stable phase of trimers may appear under certain conditions in these systems. Recent studies have started to investigate this possibility.

\subsubsection{Three-component Fermi mixtures}

For fermions of equal masses, three kinds of fermions are necessary to exhibit the Efimov effect. In the work of Paulo Bedaque and José D'Incao [397], the zerotemperature phase diagram of the equal-mass threecomponent Fermi gas is sketched out qualitatively as a function of the scattering lengths $a_{i j}$ between the different components 1, 2, 3. Their reasonning is based on how energetically favourable it is for certain components to pair, and how the condensation of these pairs at zero temperature gives different symmetries of the order parameter, corresponding to different phases. However, they note that, beyond pairing, Efimov threebody physics should also be taken into account, in particular the resonant enhancement of fermion-pair scattering at certain values of the scattering lengths $a_{i}$. According to the authors, this would result in additional phases where the pairs and fermions are spatially separated due this enhanced repulsion, instead of forming a mixture.

In his work [298], Yusuke Nishida goes one step further by explicitly considering the possibility of three fermions to form a ground-state Efimov trimer to miminise their energy. The zero temperature phase diagram, as a function of the scattering length $a$ (assumed to be the same for all pairs of components) and the three-body parameter, consists of three phases: a fully paired supefluid, a partially paired superfluid with remaining unpaired fermions, and a trimer phase, which is assumed to be a Fermi gas of ground-state trimers. Nishida calculates the boundaries of these phases in limits where they can be calculated exactly using a narrow-resonance model parameterised by $a$ and $R_{*}$ (see section 11.4):

- the high-density limit $k_{F} R_{*} \rightarrow \infty$ of the transition between the fully paired superfluid and partially paired superfluid,

- the dilute limit $k_{F} a \rightarrow 0^{-}$of the transition between the superfluid and the trimer phase at negative scattering length $a<0$, which is given by equation (11.41) indicating the appearance of a ground-state trimer

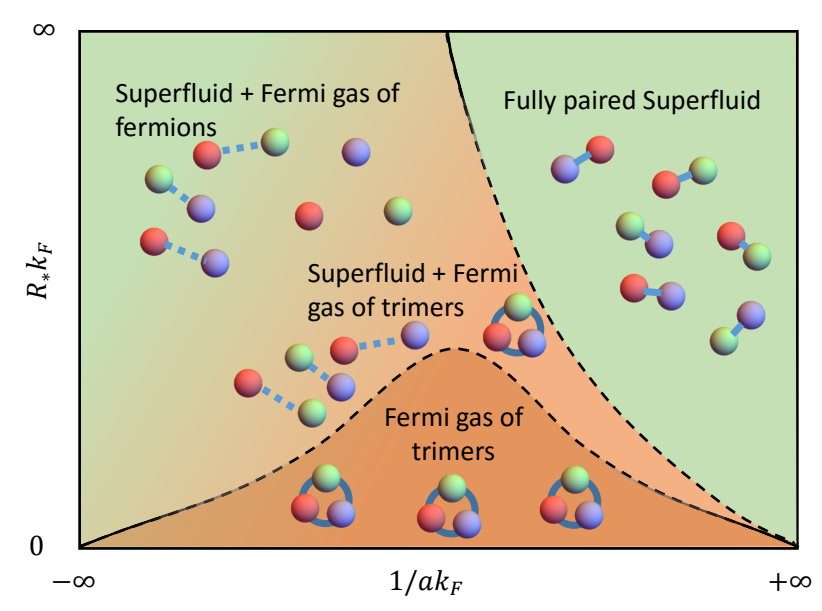

Figure 15.2: Conjectured phase diagram of the threecomponent Fermi mixture with Fermi momentum $k_{F}$, for the same narrow scattering resonance, parameterised by $a$ and $R_{*}$, between all pairs of fermions (adapted from reference [298]).

- the dilute limit $k_{F} a \rightarrow 0^{+}$of the transition between the superfluid and trimer phase at positive scattering length $a>0$, which is obtained by comparing the energies of a Fermi gas of trimers and a Bose-Einstein condensate of dimers.

Here, $k_{F}$ denotes the Fermi momentum of the system. The schematic phase diagram is represented in figure 15.2. The author confirmed the qualitative aspects of that diagram by solving a simple mean-field model including the trimers as a non-interacting Fermi gas. The author also pointed out that within the partially paired superfluid phase, there occurs a "fermiontrimer" continuity: the unpaired fermions gradually turn into trimers by binding with pairs as $k_{F} R_{*}$ is decreased. This effect is anologous to the quark-hadron continuity in nuclear matter. A qualitatively similar phase diagram is expected for broad resonances, where in general the three-body parameter $\kappa_{*}^{(0)}$ plays the role of $R_{*}^{-1}$.

We note that in a previous work [273] Yusuke Nishida also conjectured the existence of a trimer phase in a two-component Fermi gas where one component is free and the other is confined in two separate layers, making the system resemble a three-component system since the fermions confined in the different layers are distinguishable. As seen in section 9, this situation leads to the Efimov effect for one free particle resonantly interacting with two particles confined in different layers. Near this resonance, it is therefore expected that the ground-state Efimov trimer leads to a trimer phase at sufficiently low density, as in the three-component Fermi gas. 


\subsubsection{Two-component Fermi mixtures}

In mass-imbalanced two-component Fermi systems, Efimov trimers exist when the mass ratio between heavy and light fermions exceeds $\kappa_{c}^{(3)}=13.606 \ldots$, as seen in sections 6.2.2 and 6.2.5. However, it is challenging to observe many-body physics induced by these Efimov trimers in ultra-cold atom experiments, because such Efimov trimers, when made of atoms, are unstable against recombination into an atom plus a tightly bound dimer (see section 15.1). On the other hand, the universal trimers, which are remnants of the Efimov trimers in the range of mass ratios $\kappa_{1}=8.17260 \ldots<$ $M / m<\kappa_{c}^{(3)}$, are stable against three-body recombination thanks to the repulsion created by the Pauli exclusion principle (see section 6.2.5). Therefore, a gas composed of the universal trimers seems to be a promising candidate to observe a stable Efimov-induced manybody phase in ultra-cold atoms.

Such a possibility has been studied in Refs. [398] and [399]. Considering the mass ratio $\kappa_{1}<M / m<$ $8.862 \ldots$ to avoid the formation of universal tetramers (see Ref [353, 357] and section 13.2.2), a stable manybody phase of the universal trimers has been predicted to exist in a range of scattering lengths and population imbalance between heavy and light fermions. When the scattering length is small and positive so that the binding energy of the universal trimer is large enough, the trimer can be regarded as a point-like composite fermion, having three internal degrees of freedom originating from its angular momentum $L=1$. The trimer gas thus becomes a three-component Fermi gas, each component corresponding to one of the three rotational states, $m=-1,0,1$. Since the $s$-wave interaction between the universal trimers would be the dominant interaction of the trimer phase at low energy, the authors have found that its low-energy effective Hamiltonian has an SU(3) symmetry. Furthermore, in reference [399], the Resonating Group Method was used to obtain an estimate of the interaction between the two universal trimers. The interaction was found to be of the soft-core repulsion type, leading to a positive $s$-wave scattering length for the trimer-trimer scattering. This would imply that the corresponding trimer phase, in the limit of low density and low temperature, is not superfluid, but a three-component, $\mathrm{SU}(3)$ Fermi liquid [398].

We should note that the existence and nature of this trimer phase await further confirmation, since the crucial assumption about the absence of larger clusters (e.g. pentamers, hexamers) has not been precisely validated, and the method used to determine the trimertrimer interaction is only approximate. While the mass ratio window $8.17 \ldots<M / m<8.862$ seems rather restrictive, an ultra-cold mixture of ${ }^{53} \mathrm{Cr}$ and ${ }^{6} \mathrm{Li}$ atoms falls into this window $(M / m=8.80 .$.$) and is a promis-$ ing candidate to confirm the existence and investigate the properties of this trimer phase. We should note however that several points may affect the existence of a trimer phase in this mixture. First of all, the mass ratio of ${ }^{53} \mathrm{Cr}$ and ${ }^{6} \mathrm{Li}$ atoms happens to be very close to the critical mass ratio for the appearance of a universal four-body bound state. This suggests that the $p$-wave atom-trimer scattering volume is likely to be strongly enhanced in this system, similarly to the enhanced atom-dimer $p$-wave scattering observed in a fermionic ${ }^{40} \mathrm{~K}-{ }^{6} \mathrm{Li}$ mixture $[186,191,192]$. Second, below a certain scattering length, effective-range corrections and magnetic dipole-dipole interactions between ${ }^{53} \mathrm{Cr}$ atoms may not be negligible [398]. 


\section{Part VII}

\section{Conclusion}

The Efimov effect, in its most restrictive definition (see section 4.5), could be simply regarded as an oddity in the energy spectrum of three particles with shortrange interactions. On the other hand, it could be argued that the Efimov effect is on the contrary a central concept around which a wide range of stronglyinteracting systems may be described. Such is the case of long-studied systems such as the tritium nucleus or two-neutron halo nuclei in nuclear physics (see sections 4.6 and 6.4), or the triatomic molecule of helium-4 in atomic physics (see section 4.7). Even though these systems may not be recognised as Efimov states in the strictest sense, the Efimov effect provides a simple framework for the existence of such compounds. The recent experimental observations with ultra-cold atoms and theoretical developments have now opened an even richer variety of systems related to Efimov physics, from $N$-body universal clusters tied to Efimov states, to the super-Efimov effect, or mixed-dimensional Efimov states. Perhaps one of the key points of Efimov physics is to shift the paradigm of two-body correlations in pairwise interacting systems to three-body correlations due, or partly due, to the Efimov attraction. In this regard, what we have learnt from Efimov physics appears to be quite promising for the study of manybody systems where such three-body correlations may play an important role.

\section{Acknowledgments}

This work has greatly benefitted from discussions with Doerte Blume, Takumi Doi, Yasuro Funaki, Chao Gao, Chris Greene, Hui Hu, Jesper Levinsen, Haozhao Liang, Xia-Ji Liu, Sergej Moroz, Meera Parish, Dmitry Petrov, Lucas Platter, Ludovic Pricoupenko, Jean-Marc Richard, and Zheyu Shi. It was partially supported by the RIKEN iTHES project and the RIKEN Incentive Research Project.

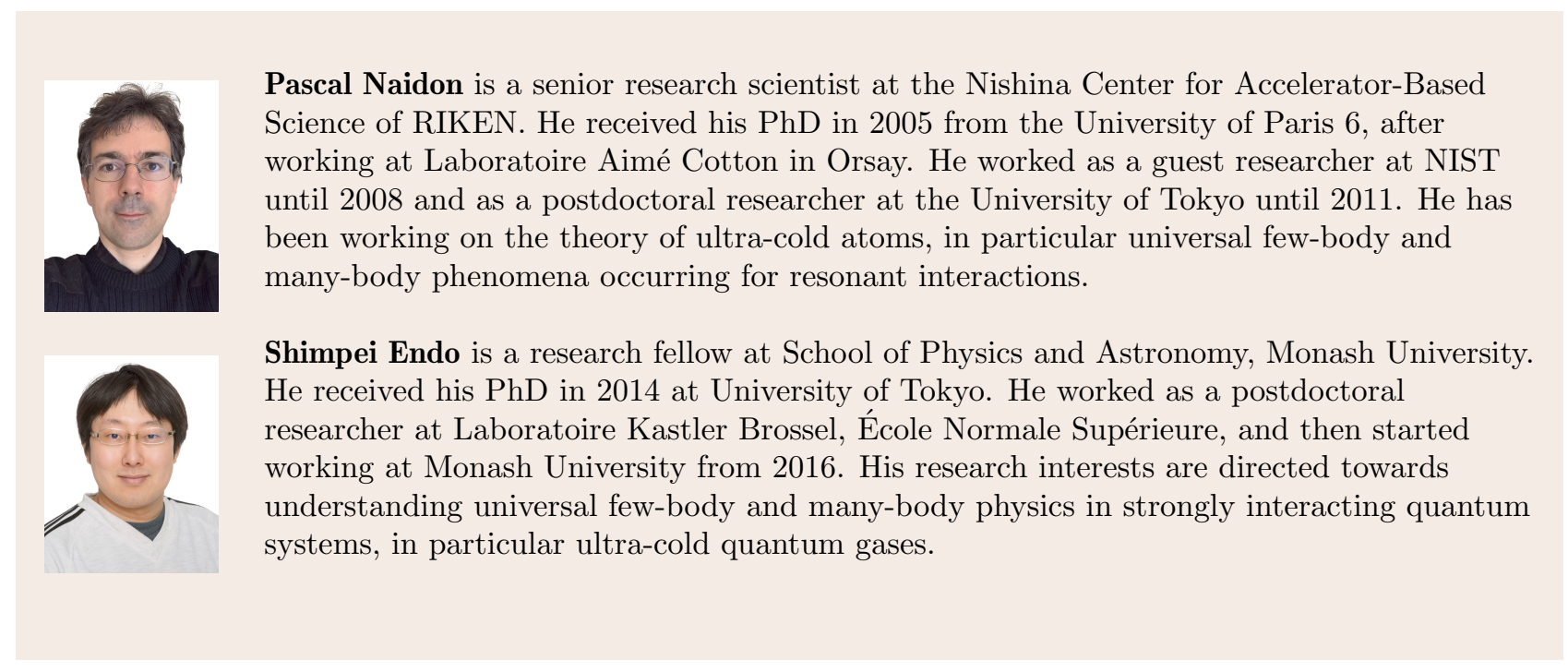




\section{Appendix: the Skorniakov - \\ Ter-Martirosian equation}

In section 4.1, the problem of three identical bosons interacting via zero-range forces was solved using the Faddeev equations in hyper-spherical coordinates. That approach has the advantage of revealing the Efimov effect in a transparent manner, and provides some analytic results. For the purpose of solving the problem numerically, however, it is often preferred to use integral equations in momentum space. These equations were first derived by G. V. Skorniakov and Karen A Ter-Martirosian [19], and take advantage of the contact nature of the interaction to reduce the dimensionality of the problem. In general, the threebody problem requires $3 d$ coordinates in $d$ dimensions. For translationally invariant systems, one can eliminate $d$ coordinates associated with the centre of mass. With contact interactions, the number of coordinates can be further reduced by $d$. Additional rotational invariance may further reduce the remaining $d$ coordinates to just one, making the problem easy to solve numerically.

It turns out that contact interactions are not necessary to obtain such simplification. It is sufficient to have a separable interaction, which enables to treat finite-range effects. In this appendix, we derive the Skorniakov - Ter-Martirosian equation for three identical bosons interacting via a separable interaction; the zero-range equation can be obtained by considering the limit when the separable interaction becomes a contact interaction.

\section{Separable interaction}

A separable interaction [80] is represented by an operator of the form:

$$
\hat{V}=\xi|\phi\rangle\langle\phi|
$$

which is a projector onto a "state" $|\phi\rangle$ multiplied by a scalar $\xi$. When applied to a two-body state $|\psi\rangle$, it gives in momentum representation:

$$
\langle\vec{p}|\hat{V}| \psi\rangle=\xi\langle\vec{p} \mid \phi\rangle\langle\phi \mid \psi\rangle=\xi \phi(\vec{p}) \int \frac{d^{3} \vec{p}}{(2 \pi)^{3}} \phi^{*}(\vec{p}) \psi(\vec{p})
$$

where $\vec{p}$ denotes the relative wave vector between two particles. Note that for later convenience, we use the same notation $\vec{p}$ for the integration variable: it should be understood that all occurences of $\vec{p}$ inside the integral refer to the integration variable. To make the choice of $\xi$ unique for a given $\hat{V}$, the function $\phi$ is normalised such that $\phi(\overrightarrow{0})=1$.
Two-body problem

The two-body Schrödinger equation in momentum space reads:

$$
\left(\frac{\hbar^{2} p^{2}}{m}-E\right) \psi(\vec{p})+\xi \phi(\vec{p})\langle\phi \mid \psi\rangle=0
$$

For scattering states $\psi_{\vec{k}}$ with an incoming wave vector $\vec{k}$, the previous equation can be written as

$$
\psi_{\vec{k}}(\vec{p})=(2 \pi)^{2} \delta^{3}(\vec{p}-\vec{k})-\frac{\frac{m}{\hbar^{2}} \xi \phi(\vec{p})}{p^{2}-k^{2}+i \varepsilon}\left\langle\phi \mid \psi_{\vec{k}}\right\rangle
$$

with $E=\hbar^{2} k^{2} / m$ and $\varepsilon \rightarrow 0^{+}$. In this equation, one can recognise the $T$-matrix element:

$$
T(\vec{k}, \vec{p})=\left\langle\vec{p}|V| \psi_{\vec{k}}\right\rangle=\xi \phi(\vec{p})\left\langle\phi \mid \psi_{\vec{k}}\right\rangle
$$

Projecting equation (15.4) onto $\phi$, one gets a closed equation for $\left\langle\phi \mid \psi_{\vec{k}}\right\rangle$ which gives the straightforward solution,

$$
\left\langle\phi \mid \psi_{\vec{k}}\right\rangle=\left(1+\frac{m}{\hbar^{2}} \xi \int \frac{d^{3} \vec{p}}{(2 \pi)^{3}} \frac{|\phi(\vec{p})|^{2}}{p^{2}-k^{2}+i \varepsilon}\right)^{-1} \phi^{*}(\vec{k}),
$$

from which one obtains $T(\vec{k}, \vec{p})$,

$T(\vec{k}, \vec{p})=\left(\frac{1}{\xi}+\frac{m}{\hbar^{2}} \int \frac{d^{3} \vec{p}^{\prime}}{(2 \pi)^{3}} \frac{\left|\phi\left(\vec{p}^{\prime}\right)\right|^{2}}{p^{\prime 2}-k^{2}+i \varepsilon}\right)^{-1} \phi(\vec{p}) \phi^{*}(\vec{k})$

This relation can be used to express the parameters $\xi$ and $\phi$ of the separable potential in terms of physical quantities such as the scattering length $a$ :

$$
\frac{4 \pi \hbar^{2}}{m} a=T(\overrightarrow{0}, \overrightarrow{0})=\left(\frac{1}{\xi}+\frac{m}{\hbar^{2}} \int \frac{d^{3} \vec{p}^{\prime}}{(2 \pi)^{3}} \frac{\left|\phi\left(\vec{p}^{\prime}\right)\right|^{2}}{p^{\prime 2}}\right)^{-1}
$$

\section{Three-body problem}

The three-body problem is expressed in a particular Jacobi wave vector set $(\vec{P}, \vec{p})$ chosen among the three possible sets $\left(\vec{P}_{k}, \vec{p}_{k}\right)$,

$$
\begin{aligned}
\vec{P}_{k} & =\frac{2}{3}\left(\vec{k}_{k}-\frac{\vec{k}_{i}+\vec{k}_{j}}{2}\right) \\
\vec{p}_{k} & =\frac{1}{2}\left(\vec{k}_{j}-\vec{k}_{i}\right)
\end{aligned}
$$

where $(i, j, k)$ is a cyclic permutation of $(1,2,3)$ and $\vec{k}_{i}$ is the wave vector of the $i$ th particle. In these coordinates, the three-body Schrödinger equation reads:

$$
\begin{gathered}
\left(\frac{3}{4} \frac{\hbar^{2}}{m} P^{2}+\frac{\hbar^{2}}{m} p^{2}-E\right) \Psi(\vec{P}, \vec{p}) \\
+\sum_{i=1,2,3} \xi \phi\left(\vec{p}_{i}\right) \int \frac{d^{3} \vec{p}_{i}}{(2 \pi)^{3}} \phi^{*}\left(\vec{p}_{i}\right) \Psi(\vec{P}, \vec{p})=0,
\end{gathered}
$$


where the first term is associated with the kinetic energy, and the second term is the sum of the action of the separable interaction on the wave function $\Psi$ over the three pairs 12,23 , and 31 . Because of the bosonic exchange symmetry, $\Psi(\vec{P}, \vec{p})$ can be replaced by $\Psi\left(\vec{P}_{i}, \vec{p}_{i}\right)$ inside the integral of equation (15.11). As in equation (15.2), it should be understood that $\vec{p}_{i}$ inside this integral refers to the integration variable, such that the integral term, once integrated, depends only the remaining Jacobi wave vector $\vec{P}_{i}$. One can thus write:

$$
\left(\frac{3}{4} P^{2}+p^{2}-\frac{m}{\hbar^{2}} E\right) \Psi(\vec{P}, \vec{p})+\sum_{i=1,2,3} F\left(\vec{P}_{i}\right) \phi\left(\vec{p}_{i}\right)=0,
$$

where

$$
F(\vec{P})=\frac{m}{\hbar^{2}} \xi \int \frac{d^{3} \vec{p}}{(2 \pi)^{3}} \phi^{*}(\vec{p}) \Psi(\vec{P}, \vec{p}) .
$$

Equation (15.12) can be inverted as

$$
\Psi(\vec{P}, \vec{p})=\Psi_{0}(\vec{P}, \vec{p})-\sum_{i=1,2,3} \frac{F\left(\vec{P}_{i}\right) \phi\left(\overrightarrow{p_{i}}\right)}{\frac{3}{4} P^{2}+p^{2}-\frac{m}{\hbar^{2}} E+i \varepsilon},
$$

where $\varepsilon \rightarrow 0^{+}$. For three-body scattering states, $E \geq 0$ and $\Psi_{0}$ is a solution of the non-interacting problem for three particles at that energy, providing the asymptotic boundary condition. For states with at least two bound particles, one has $\Psi_{0}=0$. In the remainder, we will restrict our consideration to the latter case. Inserting equation (15.14) into equation (15.13) gives:

$$
\frac{\hbar^{2}}{m \xi} F(\vec{P})=-\sum_{i=1,2,3} \int \frac{d^{3} \vec{p}}{(2 \pi)^{3}} \phi^{*}(p) \frac{F\left(\vec{P}_{i}\right) \phi\left(\vec{p}_{i}\right)}{\frac{3}{4} P^{2}+p^{2}-\frac{m}{\hbar^{2}} E+i \varepsilon}
$$

Making the choice $(\vec{P}, \vec{p})=\left(\vec{P}_{3}, \vec{p}_{3}\right)$, one can factorise one of the terms in the sum with the left-hand side of equation (15.15) as follows:

$$
\begin{aligned}
& \left(\frac{\hbar^{2}}{m \xi}+\int \frac{d^{3} \vec{p}}{(2 \pi)^{3}} \frac{|\phi(\vec{p})|^{2}}{\frac{3}{4} P^{2}+p^{2}-\frac{m}{\hbar^{2}} E+i \varepsilon}\right) F(\vec{P}) \\
& \quad+\sum_{i=1,2} \int \frac{d^{3} \vec{p}}{(2 \pi)^{3}} \phi^{*}(\vec{p}) \frac{F\left(\vec{P}_{i}\right) \phi\left(\vec{p}_{i}\right)}{\frac{3}{4} P^{2}+p^{2}-\frac{m}{\hbar^{2}} E+i \varepsilon}=0 .
\end{aligned}
$$

Expressing the Jacobi coordinate sets $\left(\vec{P}_{1}, \vec{p}_{1}\right)$ and $\left(\vec{P}_{2}, \vec{p}_{2}\right)$ in terms of $(\vec{P}, \vec{p})$, one finds

$$
\begin{aligned}
& \vec{P}_{1}=-\vec{p}-\frac{1}{2} \vec{P} \\
& \vec{p}_{1}=-\frac{1}{2} \vec{p}+\frac{3}{4} \vec{P}
\end{aligned}
$$

and

$$
\begin{aligned}
& \vec{P}_{2}=\vec{p}-\frac{1}{2} \vec{P} \\
& \vec{p}_{2}=-\frac{1}{2} \vec{p}-\frac{3}{4} \vec{P}
\end{aligned}
$$

so that $\vec{p}=-\vec{P}_{1}-\frac{1}{2} \vec{P}=\vec{P}_{2}+\frac{1}{2} \vec{P}$ and therefore $\vec{p}_{1}=$ $\frac{1}{2} \vec{P}_{1}+\vec{P}$ and $\vec{p}_{2}=-\frac{1}{2} \vec{P}_{2}-\vec{P}$. Performing a change of integration variable $\vec{p} \rightarrow \vec{P}_{1}$ and $\vec{p} \rightarrow \vec{P}_{2}$ in the first and second integrals of the sum, and relabelling the integration variable as $\vec{Q}$ in both integrals, one finally arrives at the integral equation for $F$ :

$$
\begin{aligned}
& \frac{\hbar^{2}}{m} \frac{|\phi(\vec{k})|^{2}}{T(\vec{k}, \vec{k})} F(\vec{P})+ \\
& \quad \int \frac{d^{3} \vec{Q}}{(2 \pi)^{3}} \frac{\phi^{*}\left(-\vec{Q}-\frac{\vec{P}}{2}\right) \phi\left(\frac{\vec{Q}}{2}+\vec{P}\right)+\phi^{*}\left(\vec{Q}+\frac{\vec{P}}{2}\right) \phi\left(-\frac{\vec{Q}}{2}-\vec{P}\right)}{P^{2}+Q^{2}+\vec{Q} \cdot \vec{P}-\frac{m E}{\hbar^{2}}} F(\vec{Q})
\end{aligned}
$$

where the wave vector $\vec{k}$ corresponds to the energy $\frac{\hbar^{2} k^{2}}{m}=E-\frac{3}{4} \frac{\hbar^{2} P^{2}}{m}$. This integral equation on $F$ constitutes the Skorniakov - Ter-Martirosian equation for three identical bosons interacting via a separable interaction. The remarkable point of this equation is that it replaces the original three-body Schrödinger equation (15.11) for the unknown function $\Psi(\vec{P}, \vec{p})$ of two three-dimensional variables by an equation on a function of only one three-dimensional variable, which is not possible for a general interaction. For a rotationally invariant system, the equation can be reduced to independent equations for each partial wave $F_{\ell}(P)$ that depends only on the one-dimensional variable $P=|\vec{P}|$. In order to solve equation (15.21) numerically to obtain the three-body bound states, one writes the lefthand side of equation (15.21) as a matrix acting on $F$ (through a discretisation scheme or spectral method) and looks for the energies $E<0$ that make one of its eigenvalues equal to zero, in order to satisfy the right-hand side of equation (15.21). The corresponding eigenvectors $F$ give the three-body wave functions $\Psi$ through equation (15.14).

\section{Generalisations}

The Skorniakov - Ter-Martirosian equation can be generalised to distinguishable, fermionic or any mixture of particles. In general, there are three functions $F_{i}$

$$
F_{i}\left(\vec{P}_{i}\right)=\frac{m}{\hbar^{2}} \xi \int \frac{d^{3} \vec{p}_{i}}{(2 \pi)^{3}} \phi^{*}\left(\vec{p}_{i}\right) \Psi(\vec{P}, \vec{p})
$$

to describe the three pairs of particles, and they are solutions of three coupled integral equations [245]. The interaction can also be generalised to multi-channel interaction [119, 400, 79]. There have also been generalisations to the relativistic case - see section 4.4 .

\section{Zero-range limit}

The zero-range limit can be obtained by setting $\phi(\vec{p})$ to a constant $\phi(\vec{p})=1$. In this limit, the separable potential is just a constant $\xi$ in momentum space, corresponding to a delta function in real space. However, this limit gives rise to an ultraviolet divergence of the 
integrals. These divergences can be cured by imposing a momentum cutoff $\Lambda$ and renormalising $\xi$ in terms of the physical scattering length $a$. Formally, this is equivalent to setting $\phi(\vec{p})=\theta(\Lambda-p)$ in the formulas, where $\theta$ denotes the unit step function. The integral equation (15.21) thus becomes,

$$
\begin{aligned}
& \frac{\hbar^{2}}{m} \frac{1}{T(\vec{k}, \vec{k})} F(\vec{P}) \\
& \quad+2 \int_{\substack{\left|\vec{Q}+\frac{\vec{P}}{2}\right|<\Lambda \\
\left|\vec{P}+\frac{\vec{Q}}{2}\right|<\Lambda}} \frac{d^{3} \vec{Q}}{(2 \pi)^{3}} \frac{F(\vec{Q})}{P^{2}+Q^{2}+\vec{Q} \cdot \vec{P}-\frac{m E}{\hbar^{2}}}=0 .
\end{aligned}
$$

The equation (15.8) gives the renormalisation relation:

$$
\frac{1}{a}=\frac{4 \pi \hbar^{2}}{m} \frac{1}{\xi}+\frac{2}{\pi} \Lambda
$$

From this relation, one can express the $T$-matrix elements:

$T(\vec{k}, \vec{p})=\frac{m}{4 \pi \hbar^{2}}\left(\frac{1}{a}-\frac{2}{\pi} \sqrt{-k^{2}} \arctan \frac{\Lambda}{\sqrt{-k^{2}}}\right)^{-1} \theta(\Lambda-k) \theta(\Lambda-p)$

For sufficiently large $\Lambda$, the on-shell two- body $T$ matrix element approaches

$$
T(\vec{k}, \vec{k}) \approx \frac{m}{4 \pi \hbar^{2}}\left(\frac{1}{a}-\sqrt{-k^{2}}\right)^{-1}
$$

and the restriction $\left|\vec{Q}+\frac{\vec{P}}{2}\right|<\Lambda$ and $\left|\vec{P}+\frac{\vec{Q}}{2}\right|<\Lambda$ over the integration volume can be approximated by $Q<\Lambda$. This results in the simplified equation,

$$
\begin{aligned}
& \left(\frac{1}{a}-\sqrt{\frac{3}{4} P^{2}-\frac{m}{\hbar^{2}} E}\right) F(\vec{P}) \\
& \quad+8 \pi \int_{Q<\Lambda} \frac{d^{3} \vec{Q}}{(2 \pi)^{3}} \frac{F(\vec{Q})}{P^{2}+Q^{2}+\vec{Q} \cdot \vec{P}-\frac{m E}{\hbar^{2}}}=0 .
\end{aligned}
$$

which is the original equation derived by G. V. Skorniakov and K. A. Ter-Martirosian [19]. A remarkable point of this equation is that the two-body physics enters only through the on-shell $T$-matrix elements $T(\vec{k}, \vec{k})$ and the cutoff $\Lambda$. In general, for non-separable interactions, the off-shell $T$-matrix elements $T(\vec{k}, \vec{p})$ would be required. Here, all the off-shell information is captured by the cutoff $\Lambda$, as can be checked from the expression of $T(\vec{k}, \vec{p})$ in equation (15.25). This information is however essential for the three-body problem, and $\Lambda$ cannot be set to infinity in the above integral, as the results would not converge but exhibit logarithmic oscillations with $\Lambda$. It is the large but finite value of $\Lambda$ that sets the three-body parameter of the Efimov states. Because of the discrete scale invariance discussed in the Efimov theory (see section 4.1), scaling the value of $\Lambda$ by powers of $e^{\pi /\left|s_{0}\right|}$ gives the same three-body parameters and the same observables.
It should be noted that the impossibility to take $\Lambda$ to infinity is due to the fact that only two-body interactions have been assumed. The three-body parameter may also be fixed by a three-body force. Adding a $\Lambda$-dependent zero-range three-body interaction can cancel the dependence of the observables on $\Lambda$, making it possible to take the limit $\Lambda \rightarrow \infty[53,6]$. The renormalised observables then depends on the twobody scattering length $a$ and a three-body parameter introduced by the three-body interaction.

\section{Finite-range effects}

The choice of the parameters $\xi$ and $\phi(\vec{p})$ of the separable potential in equation (15.1) depends on the system and observables of interest. In the windows of universality shown in figure 4.3 , the observables depend only the two-body scattering length $a$ and the three-body parameter $\kappa_{*}$. To calculate these universal observables, any choice of $\xi$ and $\phi(\vec{p})$ leading to a desired $a$ and $\kappa_{*}$ is possible. The most common choices are the zero-range limit $\phi(\vec{p})=\theta(\Lambda-p)$ described above, and the Gaussian separable potential with $\phi(\vec{p})=\exp \left(-\Lambda^{2} p^{2}\right)[119,400,79]$, since they lead to analytical simplifications and simple relations between the physical quantities $\left(a, \kappa_{*}\right)$ and the parameters $(\xi, \Lambda)$.

On the other hand, to calculate observables outside the universal region, that are affected by finite-range corrections, a more precise choice of the parameters is needed. As discussed in section 4.2, the interest of separable potential models over zero-range models is that they can account for the finite-range effects nonperturbatively. Indeed, separable potential models can be parameterised to reproduce exactly the two-body scattering length and effective range, i.e. reproduce exactly the low-energy two-body observables. Even when the scattering length and effective range are fixed, there remains some freedom to parameterise the separable potential, and the precise choice of the separable potential can change the value of the three-body parameter. As discussed in section 11.3, the three-body parameter is to a great extent (although not completely) set by the effective range, so that many separable potentials (such as Gaussian) having the correct scattering length and effective range already give a rough estimate of the three-body energies beyond the zero-range limit.

If one has further information on the two-body interaction, such as off-shell $T$-matrix elements or a model potential for the interaction ${ }^{20}$, one may construct an even more precise separable potential by fully exploiting its parameter space. Namely, a separable potential $\hat{V}$ can be designed to reproduce exactly an eigenstate $|\psi\rangle$ at a given energy $E$ of a given local potential $V$. It

\footnotetext{
${ }^{20}$ which are strictly speaking not two-body observables, but may be known to model accurately the interaction of certain particles.
} 
has the form [290]:

$$
\hat{V}=\frac{1}{\langle\psi|V| \psi\rangle} V|\psi\rangle\langle\psi| V
$$

which shows that the action of $\hat{V}$ onto $|\psi\rangle$ is indeed the same as $V$, thus ensuring that $|\psi\rangle$ is also an eigenstate of $\hat{V}$ at the same energy. If $|\psi\rangle$ is chosen to be the zeroenergy scattering eigenstate, the separable potential has by construction the correct scattering length and correct effective range. Indeed, the scattering length is given by the asymptotic form of the zero-energy radial wave function $\varphi(r)=r \psi(\vec{r})$ (see equation 4.8):

$$
\varphi(r) \underset{r \rightarrow \infty}{\longrightarrow} \bar{\varphi}(r)=1-\frac{r}{a}
$$

and the effective range $r_{e}$ is obtained from $\varphi$ through the formula [70],

$$
\frac{1}{2} r_{e}=\int_{0}^{\infty} d r\left[\bar{\varphi}(r)^{2}-\varphi(r)^{2}\right]
$$

In this case, the separable potential of equation (15.28) has the explicit parameterisation [39, 82]:

$$
\phi(p)=1-p \int_{0}^{\infty} d r(\bar{\varphi}(r)-\varphi(r)) \sin p r
$$

and $\xi$ is given by equation (15.8),

$$
\xi=\frac{4 \pi \hbar^{2}}{m}\left(\frac{1}{a}-\frac{2}{\pi} \int_{0}^{\infty} d p|\phi(p)|^{2}\right)^{-1}
$$

This separable representation was shown to reproduce approximately the three-body energies of the original potential [82], and in particular the three-body parameters of van der Waals potentials [39] - see section 11.2.1.

As an example, we have constructed such a separable potential for helium-4, using the zero-energy eigenstate obtained from the scaled LM2M2 potential of reference [123], as solved for the ground-state energy as a function of scattering length (varied by scaling the LM2M2 potential). The obtained energy is shown as a dotted curve in figure 4.7.

Note that the representation of a given potential in terms of a separable potential was generalised by Ernst, Shakin and Thaler (EST) [290], who have shown that any local potential $V$ can be represented exactly as a superposition of non-local separable potentials.

\section{Integral equations for the triton}

We present here the separable model of the triton, first introduced by Kharchenko [207] and used to calculate the surfaces and curves of figure 6.6. This model describes the triplet and singlet interactions of nucleons by separable potentials of the form,

$$
\begin{aligned}
& \hat{V}_{t}=\xi_{t}\left|\phi_{t}\right\rangle\left\langle\phi_{t}\right| \\
& \hat{V}_{s}=\xi_{s}\left|\phi_{s}\right\rangle\left\langle\phi_{s}\right|
\end{aligned}
$$

leading to the following integral equations:

$$
\begin{array}{r}
{\left[\frac{1}{a_{t}}+\frac{2}{\pi} \int_{0}^{\infty} d p\left|\phi_{t}(\vec{p})\right|^{2}\left(\frac{p^{2}}{\frac{3}{4} P^{2}+p^{2}-\frac{m}{\hbar^{2}} E}-1\right)\right] F_{t}(k)} \\
+4 \pi \int \frac{d^{3} \vec{Q}}{(2 \pi)^{3}} \frac{\frac{1}{2} I_{\vec{k}, \vec{k}^{\prime}}^{t t} F_{t}(Q)+\frac{3}{2} I_{\vec{k}, \vec{k}^{\prime}}^{t s} F_{s}(Q)}{P^{2}+Q^{2}+\vec{P} \cdot \vec{Q}-\frac{m E}{\hbar^{2}}}=0
\end{array}
$$

$$
\begin{array}{r}
{\left[\frac{1}{a_{s}}+\frac{2}{\pi} \int_{0}^{\infty} d p\left|\phi_{s}(\vec{p})\right|^{2}\left(\frac{p^{2}}{\frac{3}{4} P^{2}+p^{2}-\frac{m}{\hbar^{2}} E}-1\right)\right] F_{s}(k)} \\
\quad+4 \pi \int \frac{d^{3} \vec{Q}}{(2 \pi)^{3}} \frac{\frac{3}{2} I_{\vec{k}, \vec{k}^{\prime}}^{s t} F_{t}(Q)+\frac{1}{2} I_{\vec{k}, \vec{k}^{\prime}}^{s s} F_{s}(Q)}{P^{2}+Q^{2}+\vec{P} \cdot \vec{Q}-\frac{m E}{\hbar^{2}}}=0
\end{array}
$$

where

$$
I_{\vec{k}, \vec{k}^{\prime}}^{i j}=\phi_{i}^{*}\left(\left|\vec{Q}+\frac{1}{2} \vec{P}\right|\right) \phi_{j}\left(\left|\vec{P}+\frac{1}{2} \vec{Q}\right|\right)
$$

The functions $\phi_{t}$ and $\phi_{s}$ are obtained from the zeroenergy parameterisation of equation (15.29) where $\varphi(r)$ is the two-body radial wave function,

$$
\varphi(r)=\frac{r_{0}}{a}\left(\frac{Q_{\lambda}(0)}{P_{\lambda}(0)} P_{\lambda}\left(\tanh r / r_{0}\right)-Q_{\lambda}\left(\tanh r / r_{0}\right)\right)
$$

of the zero-energy scattering eigenstate for the PöschlTeller potential,

$$
V(r)=-\frac{\hbar^{2}}{m} \lambda(\lambda+1) \operatorname{sech}^{2}\left(r / r_{0}\right)
$$

with the scattering length

$$
a=r_{0}\left(\frac{Q_{\lambda}(0)}{P_{\lambda}(0)}+H_{\lambda}\right)
$$

where $P_{\lambda}(x)$ and $Q_{\lambda}(x)$ designate the Legendre polynomials and $H_{\lambda}=\int_{0}^{1} \frac{1-t^{\lambda}}{1-t} d t$ is the harmonic number, which is equal to $\sum_{n=1}^{\lambda} \frac{1}{n}$ for integral values of $\lambda$.

For $\phi_{t}$ (respectively $\phi_{s}$ ), the parameters $r_{0}$ and $\lambda$ are chosen to reproduce the triplet scattering length $a_{t}=5.4112 \mathrm{fm}$ (respectively the singlet scattering length $a_{s}=-23.7148 \mathrm{fm}$ ) and the triplet effective range $r_{e, t}=1.7436 \mathrm{fm}$ (respectively the singlet effective range $r_{e, s}=2.750 \mathrm{fm}$ ). The values are taken from reference [205]. To obtain the surfaces and curves of figure 6.6 , both $a_{t}$ and $a_{s}$ were varied away from their physical values. 


\section{References}

[1] V. Efimov, "Weakly-bound states of three resonantly-interacting particles," Yad. Fiz., 12, 1080-1091, November 1970, [Sov. J. Nucl. Phys. 12, 589-595 (1971)].

[2] V. Efimov, "Energy levels arising from resonant two-body forces in a three-body system." Physics Letters B, 33, 563 - 564, 1970.

[3] A. LeClair, J. María Román, and G. Sierra, "Russian doll renormalization group and superconductivity." Phys. Rev. B, 69, 020505, Jan 2004.

[4] E. Braaten and H.-W. Hammer, "Universality in few-body systems with large scattering length." Physics Reports, 428, 259-390, 2006.

[5] K. Wilson, "Renormalization Group and Strong Interactions." Phys. Rev. D, 3, 1818-1846, Apr 1971.

[6] P. F. Bedaque, H.-W. Hammer, and U. van Kolck, "Renormalization of the Three-Body System with Short-Range Interactions." Phys. Rev. Lett., 82, 463-467, Jan 1999.

[7] S. D. Głazek and K. G. Wilson, "Limit Cycles in Quantum Theories." Phys. Rev. Lett., 89, 230401, Nov 2002.

[8] D. Sornette, "Discrete-scale invariance and complex dimensions." Physics Reports, 297, 239 - 270, 1998.

[9] M. T. Yamashita, D. V. Fedorov, and A. S. Jensen, "Brunnian and Efimov N-Body States." Few-Body Systems, 51, 135-151, 2011.

[10] N. A. Baas, D. V. Fedorov, A. S. Jensen, K. Riisager, A. G. Volosniev, and N. T. Zinner, "Higherorder Brunnian structures and possible physical realizations," Physics of Atomic Nuclei, 77, 361368, 2014.

[11] E. Braaten and H.-W. Hammer, "Efimov physics in cold atoms." Annals of Physics, 322, 120 163, 2007, january Special Issue 2007.

[12] M. Kunitski, S. Zeller, J. Voigtsberger, A. Kalinin, L. P. H. Schmidt, M. Schöffler, A. Czasch, W. Schöllkopf, R. E. Grisenti, T. Jahnke, D. Blume, and R. Dörner, "Observation of the Efimov state of the helium trimer." Science, 348, 551-555, 2015.

[13] A. S. Jensen, K. Riisager, D. V. Fedorov, and E. Garrido, "Structure and reactions of quantum halos." Rev. Mod. Phys., 76, 215-261, Feb 2004.
[14] Y. Nishida, Y. Kato, and C. D. Batista, "Efimov effect in quantum magnets." Nature Physics, 9, 93-97, 2013.

[15] H.-W. Hammer, "Universality in QCD and Halo Nuclei," Proceedings of Science, CONFINEMENT 8, 147, 2008.

[16] H.-W. Hammer and L. Platter, "Efimov States in Nuclear and Particle Physics." Annual Review of Nuclear and Particle Science, 60, 207-236, 2010.

[17] J. Maji, S. M. Bhattacharjee, F. Seno, and A. Trovato, "When a DNA triple helix melts: an analogue of the Efimov state." New Journal of Physics, 12, 083057, 2010.

[18] L. H. Thomas, "The Interaction Between a Neutron and a Proton and the Structure of $\mathrm{H}^{3}$." Phys. Rev., 47, 903-909, Jun 1935.

[19] G. Skorniakov and K. Ter-Martirosian, "Three Body Problem for Short Range Forces. I. Scattering of Low Energy Neutrons by Deuterons," Sov. Phys. JETP, 4, 648, 1957.

[20] V. Efimov, "Level spectrum of three resonantly interacting particles," Sov. Phys. JETP Lett., 16, 34, 1972, [ZhETF Pis. Red. 16 (1972) 50].

[21] V. Efimov, "Energy levels of three resonantly interacting particles." Nuclear Physics A, 210, $157-188,1973$.

[22] R. D. Amado and J. V. Noble, "Efimov's Effect: A New Pathology of Three-Particle Systems. II." Phys. Rev. D, 5, 1992-2002, Apr 1972.

[23] T. K. Lim, "Efimov effect and spin-polarized hypernuclear-atom systems." Phys. Rev. A, 34, 4424-4425, Nov 1986.

[24] D. V. Fedorov, A. S. Jensen, and K. Riisager, "Efimov States in Halo Nuclei." Phys. Rev. Lett., 73, 2817-2820, Nov 1994.

[25] T. K. Lim, S. K. Duffy, and W. C. Damert, "Efimov State in the ${ }^{4}$ He Trimer." Phys. Rev. Lett., 38, 341-343, Feb 1977.

[26] W. Schöllkopf and J. P. Toennies, "Nondestructive Mass Selection of Small van der Waals Clusters." Science, 266, 1345-1348, 1994.

[27] R. Brühl, A. Kalinin, O. Kornilov, J. P. Toennies, G. C. Hegerfeldt, and M. Stoll, "Matter Wave Diffraction from an Inclined Transmission Grating: Searching for the Elusive ${ }^{4}$ He Trimer Efimov State." Phys. Rev. Lett., 95, 063002, Aug 2005. 
[28] E. Tiesinga, B. J. Verhaar, and H. T. C. Stoof, "Threshold and resonance phenomena in ultracold ground-state collisions." Phys. Rev. A, 47, 4114-4122, May 1993.

[29] S. Inouye, M. R. Andrews, J. Stenger, H.-J. Miesner, D. M. Stamper-Kurn, and W. Ketterle, "Observation of Feshbach resonances in a Bose-Einstein condensate," Nature, 392, 151-154, March 1998.

[30] P. Courteille, R. S. Freeland, D. J. Heinzen, F. A. van Abeelen, and B. J. Verhaar, "Observation of a Feshbach Resonance in Cold Atom Scattering." Phys. Rev. Lett., 81, 69-72, Jul 1998.

[31] B. D. Esry, C. H. Greene, and J. P. Burke, "Recombination of Three Atoms in the Ultracold Limit." Phys. Rev. Lett., 83, 1751-1754, Aug 1999.

[32] T. Kraemer, M. Mark, P. Waldburger, J. G. Danzl, C. Chin, B. Engeser, A. D. Lange, K. Pilch, A. Jaakkola, H.-C. Nägerl, and R. Grimm, "Evidence for Efimov quantum states in an ultracold gas of caesium atoms." Nature, 440, 315-318, 2006.

[33] F. Ferlaino, S. Knoop, M. Berninger, W. Harm, J. D'Incao, H.-C. Nägerl, and R. Grimm, "Evidence for Universal Four-Body States Tied to an Efimov Trimer." Phys. Rev. Lett., 102, 140401, Apr 2009.

[34] H.-W. Hammer and L. Platter, "Universal properties of the four-body system with large scattering length." The European Physical Journal A, 32, 113-120, 2007.

[35] J. von Stecher, J. P. D'Incao, and C. H. Greene, "Signatures of universal four-body phenomena and their relation to the Efimov effect." Nature Physics, 5, 417-421, 2009.

[36] N. Gross, Z. Shotan, O. Machtey, S. Kokkelmans, and L. Khaykovich, "Study of Efimov physics in two nuclear-spin sublevels of 7Li." Comptes Rendus Physique, 12, 4 - 12, 2011, few body problem - Problème à petit nombre de corps.

[37] M. Berninger, A. Zenesini, B. Huang, W. Harm, H.-C. Nägerl, F. Ferlaino, R. Grimm, P. Julienne, and J. Hutson, "Universality of the Three-Body Parameter for Efimov States in Ultracold Cesium." Phys. Rev. Lett., 107, 120401, Sep 2011.

[38] J. Wang, J. D'Incao, B. Esry, and C. Greene, "Origin of the Three-Body Parameter Universality in Efimov Physics." Phys. Rev. Lett., 108, 263001, Jun 2012.
[39] P. Naidon, S. Endo, and M. Ueda, "Physical origin of the universal three-body parameter in atomic Efimov physics." Phys. Rev. A, 90, 022106, Aug 2014.

[40] B. Huang, L. A. Sidorenkov, R. Grimm, and J. M. Hutson, "Observation of the Second Triatomic Resonance in Efimov's Scenario." Phys. Rev. Lett., 112, 190401, May 2014.

[41] S.-K. Tung, K. Jiménez-García, J. Johansen, C. V. Parker, and C. Chin, "Geometric Scaling of Efimov States in a ${ }^{6} \mathrm{Li}^{133} \mathrm{Cs}$ Mixture." Phys. Rev. Lett., 113, 240402, Dec 2014.

[42] R. Pires, J. Ulmanis, S. Häfner, M. Repp, A. Arias, E. D. Kuhnle, and M. Weidemüller, "Observation of Efimov Resonances in a Mixture with Extreme Mass Imbalance." Phys. Rev. Lett., 112, 250404, Jun 2014.

[43] N. F. Mott and H. S. W. Massey, The theory of atomic collisions. Oxford, 1965.

[44] E. Fermi, "Motion of neutrons in hydrogenous substances," Ricerca Scientifica, 7, 13-52, July 1936.

[45] S. Weinberg, The Quantum Theory Of Fields, Volume I Foundations. Cambridge University Press, 1995, chapter 12.

[46] K. A. Brueckner and K. Sawada, "Bose-Einstein Gas with Repulsive Interactions: General Theory." Phys. Rev., 106, 1117-1127, Jun 1957.

[47] A. L. Fetter and J. D. Walecka, Quantum Theory of Many-Particle Systems. Dover, 2003, chapter 10 , Section 35.

[48] K. Huang, Statistical Mechanics. New York: Wiley, 1987.

[49] H. Bethe and R. Peierls, "Quantum Theory of the Diplon," Proceedings of the Royal Society of London A: Mathematical, Physical and Engineering Sciences, 148, 146-156, 1935.

[50] D. Fedorov and A. Jensen, "Efimov effect in coordinate space Faddeev equations." Phys. Rev. Lett., 71, 4103-4106, Dec 1993.

[51] C. Wetterich, "Exact evolution equation for the effective potential." Physics Letters B, 301, 90 94, 1993.

[52] S. Moroz, S. Floerchinger, R. Schmidt, and C. Wetterich, "Efimov effect from functional renormalization." Phys. Rev. A, 79, 042705, Apr 2009.

[53] P. Bedaque, H.-W. Hammer, and U. van Kolck, "The three-boson system with shortrange interactions." Nuclear Physics A, 646, 444 - 466, 1999. 
[54] L. Faddeev, "Scattering theory for a system of three particles," Zh. Eksp. Teor. Fiz., 39, 14591467, 1961, [Sov. Phys. JETP 12, 1014 (1961)].

[55] L. Delves, "Tertiary and general-order collisions." Nuclear Physics, 9, 391 - 399, 1958-1959.

[56] R. G. Newton, Scattering Theory of Waves and Particles. Dover Publications, 2013.

[57] W. M. Frank, D. J. Land, and R. M. Spector, "Singular Potentials." Rev. Mod. Phys., 43, 36-98, Jan 1971.

[58] R. A. Minlos and L. D. Faddeev, "Comment on the problem of three particles with point interactions," JETP, 41, 1850, 1961.

[59] G. S. Danilov, "On the three-body problem with short-range forces," JETP, 40, 498, 1961, [Sov. Phys. JETP 13, 349 (1961)].

[60] V. F. Kharchenko, "Solution of the Skornyakov - Ter-Martirosyan equations for three nucleons with cutoff at large momenta," Yad. Fiz., 16, 310-315, 1972, [Sov. J. Nucl. Phys. 16, 173 (1972)].

[61] G. S. Danilov and V. I. Lebedev, "Calculation of the doublet neutron-deuteron scattering length in the theory of zero-range forces," $J$. Exptl. Theoret. Phys. (U.S.S.R.), 44, 1509-1517, May 1963, [Soviet Physics JETP, 17, 1015-1020 (1963)].

[62] G. N. J. Añaños, H. E. Camblong, C. Gorrichátegui, E. Hernández, and C. R. Ordóñez, "Anomalous commutator algebra for conformal quantum mechanics." Phys. Rev. D, 67, 045018, Feb 2003.

[63] E. Braaten, H.-W. Hammer, and M. Kusunoki, "Universal equation for Efimov states." Phys. Rev. A, 67, 022505, Feb 2003.

[64] Y. Wang, J. P. D'Incao, H.-C. Nägerl, and B. D. Esry, "Colliding Bose-Einstein Condensates to Observe Efimov Physics." Phys. Rev. Lett., 104, 113201, Mar 2010.

[65] Y. Wang and B. D. Esry, "Universal threebody physics at finite energy near Feshbach resonances." New Journal of Physics, 13, 035025, 2011.

[66] M. Thøgersen, D. V. Fedorov, and A. S. Jensen, "Universal properties of Efimov physics beyond the scattering length approximation." Phys. Rev. A, 78, 020501, Aug 2008.

[67] B. E. Grinyuk, M. V. Kuzmenko, and I. V. Simenog, "Precise study of the Efimov threeparticle spectrum and structure function within variational approach," Ukr. J. Phys., 48, 101423, 2003.

[68] L. W. Bruch and K. Sawada, "Inequality Relating the Ground-State Energies of Two and Three Bosons." Phys. Rev. Lett., 30, 25-27, Jan 1973.

[69] P. M. A. Mestrom, J. Wang, C. H. Greene, and J. P. D'Incao, "Efimov universality for ultracold atoms with positive scattering lengths," arXiv: 1609.02857, 2016.

[70] H. Bethe, "Theory of the Effective Range in Nuclear Scattering." Phys. Rev., 76, 38-50, Jul 1949.

[71] V. Efimov, "Force-range correction in the threebody problem: Application to three-nucleon systems." Phys. Rev. C, 44, 2303-2310, Dec 1991.

[72] V. Efimov, "Effective interaction of three resonantly interacting particles and the force range." Phys. Rev. C, 47, 1876-1884, May 1993.

[73] M. Thøgersen, D. V. Fedorov, A. S. Jensen, B. D. Esry, and Y. Wang, "Conditions for Efimov physics for finite-range potentials." Phys. Rev. A, 80, 013608, Jul 2009.

[74] P. K. Sorensen, D. V. Fedorov, A. S. Jensen, and N. T. Zinner, "Finite-range effects in energies and recombination rates of three identical bosons." J. Phys. B, 46, 075301, 2013.

[75] S. Nakajima, M. Horikoshi, T. Mukaiyama, P. Naidon, and M. Ueda, "Nonuniversal Efimov Atom-Dimer Resonances in a Three-Component Mixture of ${ }^{6}$ Li." Phys. Rev. Lett., 105, 023201 , Jul 2010.

[76] P. Massignan and H. T. C. Stoof, "Efimov states near a Feshbach resonance." Phys. Rev. A, 78, 030701, Sep 2008.

[77] D. Petrov, "Three-Boson Problem near a Narrow Feshbach Resonance." Phys. Rev. Lett., 93, 143201, Sep 2004.

[78] S. Nakajima, M. Horikoshi, T. Mukaiyama, P. Naidon, and M. Ueda, "Measurement of an Efimov Trimer Binding Energy in a ThreeComponent Mixture of ${ }^{6} \mathrm{Li} . "$ Phys. Rev. Lett., 106, 143201, Apr 2011.

[79] P. Naidon and M. Ueda, "The Efimov effect in lithium 6." Comptes Rendus Physique, 12, 13 26, 2011, few body problem - Problème à petit nombre de corps.

[80] Y. Yamaguchi, "Two-Nucleon Problem When the Potential Is Nonlocal but Separable. I." Phys. Rev., 95, 1628-1634, Sep 1954. 
[81] P. Naidon, E. Hiyama, and M. Ueda, "Universality and the three-body parameter of ${ }^{4} \mathrm{He}$ trimers." Phys. Rev. A, 86, 012502, Jul 2012.

[82] P. Naidon, S. Endo, and M. Ueda, "Microscopic Origin and Universality Classes of the Efimov Three-Body Parameter." Phys. Rev. Lett., 112, 105301, Mar 2014.

[83] L. Platter, C. Ji, and D. R. Phillips, "Range corrections to three-body observables near a Feshbach resonance." Phys. Rev. A, 79, 022702, Feb 2009.

[84] C. Ji, D. R. Phillips, and L. Platter, "Beyond universality in three-body recombination: An effective field theory treatment." EPL (Europhysics Letters), 92, 13003, 2010.

[85] L. Platter and D. R. Phillips, "The Three-Boson System at Next-To-Next-To-Leading Order." Few Body Syst., 40, 35-55, 2006.

[86] L. Platter, "Three-nucleon system at next-tonext-to-leading order." Phys. Rev. C, 74, 037001, Sep 2006.

[87] C. Ji, D. R. Phillips, and L. Platter, "The three-boson system at next-to-leading order in an effective field theory for systems with a large scattering length." Annals of Physics, 327, 1803 - 1824, 2012, july 2012 Special Issue.

[88] A. Kievsky and M. Gattobigio, "Universal nature and finite-range corrections in elastic atom-dimer scattering below the dimer breakup threshold." Phys. Rev. A, 87, 052719, May 2013.

[89] E. Garrido, M. Gattobigio, and A. Kievsky, "Recombination rates from potential models close to the unitary limit." Phys. Rev. A, 88, 032701, Sep 2013.

[90] A. Kievsky, N. K. Timofeyuk, and M. Gattobigio, " $N$-boson spectrum from a discrete scale invariance." Phys. Rev. A, 90, 032504, Sep 2014.

[91] C. Ji, E. Braaten, D. R. Phillips, and L. Platter, "Universal relations for range corrections to Efimov features." Phys. Rev. A, 92, 030702, Sep 2015 .

[92] A. Deltuva, A. C. Fonseca, and P. U. Sauer, "Momentum-space treatment of the Coulomb interaction in three-nucleon reactions with two protons." Phys. Rev. C, 71, 054005, May 2005.

[93] H.-W. Hammer and R. Higa, "A model study of discrete scale invariance and long-range interactions." The European Physical Journal A, 37, 193-200, 2008.
[94] A. Griesmaier, J. Werner, S. Hensler, J. Stuhler, and T. Pfau, "Bose-Einstein Condensation of Chromium." Phys. Rev. Lett., 94, 160401, Apr 2005.

[95] J. Stuhler, A. Griesmaier, T. Koch, M. Fattori, T. Pfau, S. Giovanazzi, P. Pedri, and L. Santos, "Observation of Dipole-Dipole Interaction in a Degenerate Quantum Gas." Phys. Rev. Lett., 95, 150406, Oct 2005.

[96] M. Lu, N. Q. Burdick, S. H. Youn, and B. L. Lev, "Strongly Dipolar Bose-Einstein Condensate of Dysprosium." Phys. Rev. Lett., 107, 190401, Oct 2011.

[97] K. Aikawa, A. Frisch, M. Mark, S. Baier, A. Rietzler, R. Grimm, and F. Ferlaino, "BoseEinstein Condensation of Erbium." Phys. Rev. Lett., 108, 210401, May 2012.

[98] A. Frisch, M. Mark, K. Aikawa, F. Ferlaino, J. L. Bohn, C. Makrides, A. Petrov, and S. Kotochigova, "Quantum chaos in ultracold collisions of gas-phase erbium atoms," Nature, 507, 475$479,2014$.

[99] J. M. Doyle, B. Friedrich, J. Kim, and D. Patterson, "Buffer-gas loading of atoms and molecules into a magnetic trap." Phys. Rev. A, 52, R2515-R2518, Oct 1995.

[100] K.-K. Ni, S. Ospelkaus, D. Wang, G. Quéméner, B. Neyenhuis, M. De Miranda, J. Bohn, J. Ye, and D. Jin, "Dipolar collisions of polar molecules in the quantum regime," Nature, 464, 1324-1328, 2010 .

[101] B. Neyenhuis, B. Yan, S. A. Moses, J. P. Covey, A. Chotia, A. Petrov, S. Kotochigova, J. Ye, and D. S. Jin, "Anisotropic Polarizability of Ultracold Polar ${ }^{40} \mathrm{~K}^{87} \mathrm{Rb}$ Molecules." Phys. Rev. Lett., 109, 230403, Dec 2012.

[102] M. Marinescu and L. You, "Controlling AtomAtom Interaction at Ultralow Temperatures by dc Electric Fields." Phys. Rev. Lett., 81, 4596-4599, Nov 1998.

[103] V. Roudnev and M. Cavagnero, "Universal resonant ultracold molecular scattering." Phys. Rev. A, 79, 014701, Jan 2009.

[104] C. Ticknor and S. T. Rittenhouse, "Three Body Recombination of Ultracold Dipoles to Weakly Bound Dimers." Phys. Rev. Lett., 105, 013201, Jul 2010.

[105] J. L. Bohn, M. Cavagnero, and C. Ticknor, "Quasi-universal dipolar scattering in cold and ultracold gases." New Journal of Physics, 11, 055039, 2009. 
[106] Y. Wang, J. D'Incao, and C. Greene, "Efimov Effect for Three Interacting Bosonic Dipoles." Phys. Rev. Lett., 106, 233201, Jun 2011.

[107] Y. Wang, J. P. D'Incao, and C. H. Greene, "Universal Three-Body Physics for Fermionic Dipoles." Phys. Rev. Lett., 107, 233201, Nov 2011.

[108] S. Moroz, J. P. D'Incao, and D. S. Petrov, "Generalized Efimov Effect in One Dimension." Phys. Rev. Lett., 115, 180406, Oct 2015.

[109] N. L. Guevara, Y. Wang, and B. D. Esry, "New Class of Three-Body States." Phys. Rev. Lett., 108, 213202, May 2012.

[110] F. Calogero, "Solution of a Three-Body Problem in One Dimension." Journal of Mathematical Physics, 10, 2191-2196, 1969.

[111] Y. Castin, C. Mora, and L. Pricoupenko, "Four-Body Efimov Effect for Three Fermions and a Lighter Particle." Phys. Rev. Lett., 105, 223201, Nov 2010.

[112] J. V. Lindesey and H. P. Noyer, "Minimal Relativistic Three-Particle Equation and On the relativistic Efimov effect." SLAC-PUB, 2515, 1980 .

[113] J. V. Lindesey and H. P. Noyer, "Zero Range Scattering Theory II. Minimal Relativistic Three-Particle Equation and The Efimov effect." SLAC-PUB, 2932, 1986.

[114] T. Frederico, "Null-plane model of three bosons with zero-range interaction." Physics Letters B, 282, $409-414,1992$.

[115] J. Carbonell and V. A. Karmanov, "Three-boson relativistic bound states with zero-range twobody interaction." Phys. Rev. C, 67, 037001, Mar 2003.

[116] T. Frederico and G. Salmè, "Projecting the Bethe-Salpeter Equation onto the Light-Front and Back: A Short Review." Few-Body Systems, 49, 163-175, 2011.

[117] B. D. Esry, C. D. Lin, and C. H. Greene, "Adiabatic hyperspherical study of the helium trimer." Phys. Rev. A, 54, 394-401, Jul 1996.

[118] P. Giannakeas and C. H. Greene, "Van der Waals Universality in Homonuclear Atom-Dimer Elastic Collisions." Few-Body Systems, 58, 20, 2016.

[119] M. Lee, T. Köhler, and P. Julienne, "Excited Thomas-Efimov levels in ultracold gases." Phys. Rev. A, 76, 012720, Jul 2007.
[120] F. Werner, "Efimov states with strong threebody losses." EPL (Europhysics Letters), 86, 66006, 2009.

[121] J. Voigtsberger, S. Zeller, J. Becht, N. Neumann, F. Sturm, H.-K. Kim, M. Waitz, F. Trinter, M. Kunitski, A. Kalinin, J. Wu, W. Schöllkopf, D. Bressanini, A. Czasch, J. B. Williams, K. Ullmann-Pfleger, L. P. H. Schmidt, M. S. Schöffler, R. E. Grisenti, T. Jahnke, and R. Dörner, "Imaging the structure of the trimer systems ${ }^{4} \mathrm{He}_{3}$ and ${ }^{3} \mathrm{He}^{4} \mathrm{He}_{2}$." Nature Communications, 5, 2014.

[122] R. Higa, H.-W. Hammer, and U. van Kolck, " $\alpha \alpha$ scattering in halo effective field theory." Nuclear Physics A, 809, 171 - 188, 2008.

[123] R. A. Aziz and M. J. Slaman, "An examination of abinitio results for the helium potential energy curve." The Journal of Chemical Physics, 94, 8047-8053, 1991.

[124] S. Ali and A. Bodmer, "Phenomenological $\alpha-\alpha$ potentials." Nuclear Physics, 80, 99 - 112, 1966.

[125] H. Suno, Y. Suzuki, and P. Descouvemont, "Triple $-\alpha$ continuum structure and Hoyle resonance of ${ }^{12} \mathrm{C}$ using the hyperspherical slow variable discretization." Phys. Rev. C, 91, 014004, Jan 2015.

[126] C. Chin, R. Grimm, P. Julienne, and E. Tiesinga, "Feshbach resonances in ultracold gases." Rev. Mod. Phys., 82, 1225-1286, Apr 2010.

[127] T. Cornelius and W. Glöckle, "Efimov states for three 4He atoms?" The Journal of Chemical Physics, 85, 3906-3912, 1986.

[128] E. A. Kolganova, A. K. Motovilov, and W. Sandhas, "The $4 \mathrm{He}$ Trimer as an Efimov System." Few-Body Systems, 51, 249-257, 2011.

[129] H. Feshbach, "Unified theory of nuclear reactions." Annals of Physics, 5, 357 - 390, 1958 .

[130] H. Feshbach, "A unified theory of nuclear reactions. $\{\mathrm{II}\} . "$ Annals of Physics, 19, 287 $313,1962$.

[131] U. Fano, "Effects of Configuration Interaction on Intensities and Phase Shifts." Phys. Rev., 124, 1866-1878, Dec 1961.

[132] F. Ferlaino, A. Zenesini, M. Berninger, B. Huang, H.-C. Nägerl, and R. Grimm, "Efimov Resonances in Ultracold Quantum Gases." Few-Body Syst., 51, 113-133, 2011.

[133] Y. Wang and B. D. Esry, "Efimov Trimer Formation via Ultracold Four-Body Recombination." Phys. Rev. Lett., 102, 133201, Mar 2009. 
[134] E. Braaten and H.-W. Hammer, "Three-Body Recombination into Deep Bound States in a Bose Gas with Large Scattering Length." Phys. Rev. Lett., 87, 160407, Oct 2001.

[135] E. Braaten and H.-W. Hammer, "Enhanced dimer relaxation in an atomic and molecular Bose-Einstein condensate." Phys. Rev. A, 70, 042706, Oct 2004.

[136] E. Braaten, H.-W. Hammer, D. Kang, and L. Platter, "Three-body recombination of identical bosons with a large positive scattering length at nonzero temperature." Phys. Rev. A, 78, 043605 , Oct 2008.

[137] B. S. Rem, A. T. Grier, I. Ferrier-Barbut, U. Eismann, T. Langen, N. Navon, L. Khaykovich, F. Werner, D. S. Petrov, F. Chevy, and C. Salomon, "Lifetime of the Bose Gas with Resonant Interactions." Phys. Rev. Lett., 110, 163202, Apr 2013.

[138] S. Knoop, F. Ferlaino, M. Mark, M. Berninger, H. Schoebel, H.-C. Naegerl, and R. Grimm, "Observation of an Efimov-like trimer resonance in ultracold atom-dimer scattering." Nature Physics, 5, 227, 2009.

[139] M. Zaccanti, B. Deissler, C. D'Errico, M. Fattori, M. Jona-Lasinio, S. Müller, G. Roati, M. Inguscio, and G. Modugno, "Observation of an Efimov spectrum in an atomic system." Nature Physics, 5, 586-591, 2009.

[140] S. E. Pollack, D. Dries, and R. G. Hulet, "Universality in Three- and Four-Body Bound States of Ultracold Atoms." Science, 326, 1683-1685, 2009.

[141] S. Roy, M. Landini, A. Trenkwalder, G. Semeghini, G. Spagnolli, A. Simoni, M. Fattori, M. Inguscio, and G. Modugno, "Test of the Universality of the Three-Body Efimov Parameter at Narrow Feshbach Resonances." Phys. Rev. Lett., 111, 053202, Aug 2013.

[142] P. Dyke, S. E. Pollack, and R. G. Hulet, "Finiterange corrections near a Feshbach resonance and their role in the Efimov effect." Phys. Rev. A, 88, 023625, Aug 2013.

[143] N. Gross, Z. Shotan, S. Kokkelmans, and L. Khaykovich, "Observation of Universality in Ultracold ${ }^{7} \mathrm{Li}$ Three-Body Recombination." Phys. Rev. Lett., 103, 163202, Oct 2009.

[144] M. Stoll and T. Köhler, "Production of threebody Efimov molecules in an optical lattice." Phys. Rev. A, 72, 022714, Aug 2005.
[145] O. Machtey, Z. Shotan, N. Gross, and L. Khaykovich, "Association of Efimov Trimers from a Three-Atom Continuum." Phys. Rev. Lett., 108, 210406, May 2012.

[146] Y. Nishida, "Electron spin resonance in a dilute magnon gas as a probe of magnon scattering resonances." Phys. Rev. B, 88, 224402, Dec 2013.

[147] Y. Nishida, S. Moroz, and D. T. Son, "Super Efimov Effect of Resonantly Interacting Fermions in Two Dimensions." Phys. Rev. Lett., 110, 235301, Jun 2013.

[148] J. Omachi, T. Suzuki, K. Kato, N. Naka, K. Yoshioka, and M. Kuwata-Gonokami, "Observation of Excitonic $N$-Body Bound States: Polyexcitons in Diamond." Phys. Rev. Lett., 111, 026402, Jul 2013.

[149] H. Watanabe and H. Murayama, "Unified Description of Nambu-Goldstone Bosons without Lorentz Invariance." Phys. Rev. Lett., 108, 251602, Jun 2012.

[150] J. Frenkel, "On the Transformation of light into Heat in Solids. I." Phys. Rev., 37, 17-44, Jan 1931.

[151] G. H. Wannier, "The Structure of Electronic Excitation Levels in Insulating Crystals." Phys. Rev., 52, 191-197, Aug 1937.

[152] K. Yoshioka, E. Chae, and M. KuwataGonokami, "Transition to a Bose-Einstein condensate and relaxation explosion of excitons at sub-Kelvin temperatures," Nature Communications, 2, 328, 2011.

[153] A. G. Steele, W. G. McMullan, and M. L. W. Thewalt, "Discovery of Polyexcitons." Phys. Rev. Lett., 59, 2899-2902, Dec 1987.

[154] M. Thewalt, V. Karasyuk, D. Harrison, and D. Huber, "Near-Infrared Photoluminescence of Polyexcitons in Silicon," Proceedings of the 23rd ICPS, 1, 341, 1996.

[155] C. A. Regal, C. Ticknor, J. L. Bohn, and D. S. Jin, "Tuning $p$-Wave Interactions in an Ultracold Fermi Gas of Atoms." Phys. Rev. Lett., 90, 053201, Feb 2003.

[156] J. Zhang, E. G. M. van Kempen, T. Bourdel, L. Khaykovich, J. Cubizolles, F. Chevy, M. Teichmann, L. Tarruell, S. J. J. M. F. Kokkelmans, and C. Salomon, "P-wave Feshbach resonances of ultracold ${ }^{6}$ Li." Phys. Rev. A, 70, 030702, Sep 2004. 
[157] C. H. Schunck, M. W. Zwierlein, C. A. Stan, S. M. F. Raupach, W. Ketterle, A. Simoni, E. Tiesinga, C. J. Williams, and P. S. Julienne, "Feshbach resonances in fermionic ${ }^{6} \mathrm{Li} . "$ Phys. Rev. A, 71, 045601, Apr 2005.

[158] T. Nakasuji, J. Yoshida, and T. Mukaiyama, "Experimental determination of $p$-wave scattering parameters in ultracold ${ }^{6} \mathrm{Li}$ atoms." Phys. Rev. A, 88, 012710, Jul 2013.

[159] J. H. Macek and J. Sternberg, "Properties of Pseudopotentials for Higher Partial Waves." Phys. Rev. Lett., 97, 023201, Jul 2006.

[160] E. Braaten, P. Hagen, H.-W. Hammer, and L. Platter, "Renormalization in the three-body problem with resonant $p$-wave interactions." Phys. Rev. A, 86, 012711, Jul 2012.

[161] Y. Nishida, "Impossibility of the Efimov effect for $p$-wave interactions." Phys. Rev. A, 86, 012710, Jul 2012.

[162] M. Jona-Lasinio, L. Pricoupenko, and Y. Castin, "Three fully polarized fermions close to a $p$-wave Feshbach resonance." Phys. Rev. A, 77, 043611, Apr 2008.

[163] L. Pricoupenko, "Modeling Interactions for Resonant p-Wave Scattering." Phys. Rev. Lett., 96, 050401, Feb 2006.

[164] H.-W. Hammer and D. Lee, "Causality and universality in low-energy quantum scattering," Physics Letters B, 681, 500-503, 2009.

[165] H.-W. Hammer and D. Lee, "Causality and the effective range expansion," Annals of Physics, 325, 2212-2233, 2010.

[166] J. Levinsen, N. R. Cooper, and V. Gurarie, "Strongly Resonant p-Wave Superfluids." Phys. Rev. Lett., 99, 210402, Nov 2007.

[167] V. Gurarie, L. Radzihovsky, and A. V. Andreev, "Quantum Phase Transitions across a $p$-Wave Feshbach Resonance." Phys. Rev. Lett., 94, 230403, Jun 2005.

[168] C.-H. Cheng and S.-K. Yip, "Anisotropic Fermi Superfluid via $p$-Wave Feshbach Resonance." Phys. Rev. Lett., 95, 070404, Aug 2005.

[169] V. Gurarie and L. Radzihovsky, "Resonantly paired fermionic superfluids," Annals of Physics, 322, 2-119, 2007.

[170] J. Levinsen, N. R. Cooper, and V. Gurarie, "Stability of fermionic gases close to a $p$-wave Feshbach resonance." Phys. Rev. A, 78, 063616, Dec 2008.
[171] H. Suno, B. D. Esry, and C. H. Greene, "Recombination of Three Ultracold Fermionic Atoms." Phys. Rev. Lett., 90, 053202, Feb 2003.

[172] A. G. Volosniev, D. V. Fedorov, A. S. Jensen, and N. T. Zinner, "Borromean ground state of fermions in two dimensions." Journal of Physics B: Atomic, Molecular and Optical Physics, 47, 185302, 2014.

[173] C. Gao, J. Wang, and Z. Yu, "Revealing the origin of super Efimov states in the hyperspherical formalism." Phys. Rev. A, 92, 020504, Aug 2015.

[174] D. K. Gridnev, "Three resonating fermions in flatland: proof of the super Efimov effect and the exact discrete spectrum asymptotics," Journal of Physics A: Mathematical and Theoretical, 47, 505204, 2014.

[175] C. Gao and Z. Yu, "Three Identical Fermions with Resonant p-wave Interactions in Two Dimensions." arXiv:1401.0965, 2014.

[176] S. Moroz and Y. Nishida, "Super Efimov effect for mass-imbalanced systems." Phys. Rev. A, 90, 063631, Dec 2014.

[177] M. A. Efremov and W. P. Schleich, "QuasiCoulomb series in a two-dimensional three-body system." arXiv:1407.3352, 2014.

[178] P. Kornilovitch, "Ferromagnetism and Borromean Binding in Three-Fermion Clusters." Phys. Rev. Lett., 112, 077202, Feb 2014.

[179] B. Acharya, C. Ji, and L. Platter, "Effectivefield-theory analysis of Efimov physics in heteronuclear mixtures of ultracold atomic gases." Phys. Rev. A, 94, 032702, Sep 2016.

[180] K. Helfrich, H.-W. Hammer, and D. S. Petrov, "Three-body problem in heteronuclear mixtures with resonant interspecies interaction." Phys. Rev. A, 81, 042715, Apr 2010.

[181] D. S. Petrov and F. Werner, "Three-body recombination in heteronuclear mixtures at finite temperature." Phys. Rev. A, 92, 022704, Aug 2015.

[182] M. Mikkelsen, A. S. Jensen, D. V. Fedorov, and N. T. Zinner, "Three-body recombination of two-component cold atomic gases into deep dimers in an optical model." Journal of Physics B: Atomic, Molecular and Optical Physics, 48, 085301, 2015.

[183] O. I. Kartavtsev and A. V. Malykh, "Low-energy three-body dynamics in binary quantum gases." Journal of Physics B: Atomic, Molecular and Optical Physics, 40, 1429, 2007. 
[184] E. Nielsen, D. Fedorov, A. Jensen, and E. Garrido, "The three-body problem with short-range interactions." Physics Reports, 347, $373-459,2001$

[185] J. P. D'Incao and B. D. Esry, "Mass dependence of ultracold three-body collision rates." Phys. Rev. A, 73, 030702, Mar 2006.

[186] S. Endo, P. Naidon, and M. Ueda, "Universal Physics of 2+1 Particles with Non-Zero Angular Momentum." Few-Body Systems, 51, 207-217, 2011.

[187] K. Helfrich and H.-W. Hammer, "On the Efimov effect in higher partial waves." Journal of Physics B: Atomic, Molecular and Optical Physics, 44, 215301, 2011.

[188] O. Kartavtsev and A. Malykh, "Universal description of the rotational-vibrational spectrum of three particles with zero-range interactions." JETP Letters, 86, 625-629, 2008.

[189] A. C. Fonseca, E. F. Redish, and P. Shanley, "Efimov effect in an analytically solvable model." Nuclear Physics A, 320, 273 - 288, 1979.

[190] D. S. Petrov, "Three-body problem in Fermi gases with short-range interparticle interaction." Phys. Rev. A, 67, 010703, Jan 2003.

[191] J. Levinsen and D. Petrov, "Atom-dimer and dimer-dimer scattering in fermionic mixtures near a narrow Feshbach resonance," The European Physical Journal D, 65, 67-82, 2011.

[192] M. Jag, M. Zaccanti, M. Cetina, R. S. Lous, F. Schreck, R. Grimm, D. S. Petrov, and J. Levinsen, "Observation of a Strong Atom-Dimer Attraction in a Mass-Imbalanced Fermi-Fermi Mixture." Phys. Rev. Lett., 112, 075302 , Feb 2014.

[193] S. Endo, P. Naidon, and M. Ueda, "Crossover trimers connecting continuous and discrete scaling regimes." Phys. Rev. A, 86, 062703, Dec 2012.

[194] A. Safavi-Naini, S. T. Rittenhouse, D. Blume, and H. R. Sadeghpour, "Nonuniversal bound states of two identical heavy fermions and one light particle." Phys. Rev. A, 87, 032713, Mar 2013.

[195] Y. Nishida, D. T. Son, and S. Tan, "Universal Fermi Gas with Two- and Three-Body Resonances." Phys. Rev. Lett., 100, 090405, Mar 2008.

[196] O. I. Kartavtsev and A. V. Malykh, "Universal description of three two-component fermions." EPL (Europhysics Letters), 115, 36005, 2016.
[197] A. Bulgac and V. Efimov, "Spin dependence of the level spectrum of three resonantly interacting particles," Sov. J. Nucl. Phys., 22, 153, 1976, [Yad. Fiz. 22 (1975) 296].

[198] V. Galitski and I. B. Spielman, "Spin-orbit coupling in quantum gases." Nature, 494, 49-54, February 2013.

[199] Z.-Y. Shi, X. Cui, and H. Zhai, "Universal Trimers Induced by Spin-Orbit Coupling in Ultracold Fermi Gases." Phys. Rev. Lett., 112, 013201, Jan 2014.

[200] Z.-Y. Shi, H. Zhai, and X. Cui, "Efimov physics and universal trimers in spin-orbitcoupled ultracold atomic mixtures." Phys. Rev. A, 91, 023618, Feb 2015.

[201] A. G. Sitenko and V. F. Kharchenko, "On the binding and scattering of the three nucleon system," Nuclear Physics, 49, 15-28, 1963.

[202] V. Efimov and E. Tkachenko, "Explanation of the Phillips line in the three-nucleon problem." Physics Letters B, 157, 108 - 114, 1985.

[203] V. Efimov and E. Tkachenko, "On the correlation between the triton binding energy and the neutron-deuteron doublet scattering length." Few-Body Systems, 4, 71-88, 1988.

[204] P. F. Bedaque, G. Rupak, H. W. Grießhammer, and H.-W. Hammer, "Low energy expansion in the three body system to all orders and the triton channel." Nuclear Physics A, 714, 589 610, 2003.

[205] R. W. Hackenburg, "Neutron-proton effective range parameters and zero-energy shape dependence." Phys. Rev. C, 73, 044002, Apr 2006.

[206] A. Phillips, "Consistency of the low-energy three-nucleon observables and the separable interaction model." Nuclear Physics A, 107, 209 - 216, 1968.

[207] V. Kharchenko, N. Petrov, and S. Storozhenko, "Binding energy of triton and n-d scattering lengths with separable potentials." Nuclear Physics A, 106, 464-475, 1967.

[208] T. Frederico, "Universal Aspects of Neutron Halos in Light Exotic Nuclei." Few-Body Systems, $55,651-657,2014$.

[209] I. Tanihata, H. Savajols, and R. Kanungo, "Recent experimental progress in nuclear halo structure studies." Progress in Particle and Nuclear Physics, 68, 215 - 313, 2013. 
[210] C. Ji, C. Elster, and D. R. Phillips, " ${ }^{6}$ He nucleus in halo effective field theory." Phys. Rev. C, 90, 044004, Oct 2014.

[211] S. Dasgupta, I. Mazumdar, and V. S. Bhasin, "Three-body model for ${ }^{11} \mathrm{Li}$ with separable potentials." Phys. Rev. C, 50, R550-R554, Aug 1994.

[212] I. Mazumdar and V. S. Bhasin, "Efimov effect in the nuclear halo ${ }^{14}$ Be nucleus." Phys. Rev. C, 56, R5-R7, Jul 1997.

[213] I. Mazumdar, V. Arora, and V. S. Bhasin, "Three-body analysis of the occurrence of Efimov states in $2 n$ halo nuclei such as ${ }^{19} \mathrm{~B},{ }^{22} \mathrm{C}$, and ${ }^{20}$ C." Phys. Rev. C, 61, 051303, Apr 2000.

[214] I. Mazumdar, A. R. P. Rau, and V. S. Bhasin, "Efimov States and their Fano Resonances in a Neutron-Rich Nucleus." Phys. Rev. Lett., 97, 062503, Aug 2006.

[215] A. E. A. Amorim, T. Frederico, and L. Tomio, "Universal aspects of Efimov states and light halo nuclei." Phys. Rev. C, 56, R2378-R2381, Nov 1997.

[216] M. Yamashita, T. Frederico, and L. Tomio, "Trajectory of neutron-neutron- ${ }^{18} \mathrm{C}$ excited threebody state." Physics Letters B, 660, 339 - 344, 2008.

[217] M. Yamashita, T. Frederico, and L. Tomio, "Neutron- ${ }^{19} \mathrm{C}$ scattering near an Efimov state." Physics Letters B, 670, 49 - 54, 2008.

[218] D. L. Canham and H.-W. Hammer, "Universal properties and structure of halo nuclei." Eur. Phys. J. A, 37, 367-380, 2008.

[219] D. L. Canham and H.-W. Hammer, "Range corrections for two-neutron halo nuclei in effective theory." Nuclear Physics A, 836, 275 292, 2010.

[220] K. Tanaka, T. Yamaguchi, T. Suzuki, T. Ohtsubo, M. Fukuda, D. Nishimura, M. Takechi, K. Ogata, A. Ozawa, T. Izumikawa, T. Aiba, N. Aoi, H. Baba, Y. Hashizume, K. Inafuku, N. Iwasa, K. Kobayashi, M. Komuro, Y. Kondo, T. Kubo, M. Kurokawa, T. Matsuyama, S. Michimasa, T. Motobayashi, T. Nakabayashi, S. Nakajima, T. Nakamura, H. Sakurai, R. Shinoda, M. Shinohara, H. Suzuki, E. Takeshita, S. Takeuchi, Y. Togano, K. Yamada, T. Yasuno, and M. Yoshitake, "Observation of a Large Reaction Cross Section in the Drip-Line Nucleus ${ }^{22}$ C." Phys. Rev. Lett., 104, 062701, Feb 2010.

[221] Y. Togano, T. Nakamura, Y. Kondo, J. Tostevin, A. Saito, J. Gibelin, N. Orr, N. Achouri, T. Aumann, H. Baba, F. Delaunay, P. Doornenbal,
N. Fukuda, J. Hwang, N. Inabe, T. Isobe, D. Kameda, D. Kanno, S. Kim, N. Kobayashi, T. Kobayashi, T. Kubo, S. Leblond, J. Lee, F. Marqués, R. Minakata, T. Motobayashi, D. Murai, T. Murakami, K. Muto, T. Nakashima, N. Nakatsuka, A. Navin, S. Nishi, S. Ogoshi, H. Otsu, H. Sato, Y. Satou, Y. Shimizu, H. Suzuki, K. Takahashi, H. Takeda, S. Takeuchi, R. Tanaka, A. Tuff, M. Vandebrouck, and K. Yoneda, "Interaction cross section study of the two-neutron halo nucleus 22C." Physics Letters B, 761, 412 - 418, 2016.

[222] M. Yamashita, R. M. de Carvalho, T. Frederico, and L. Tomio, "Constraints on two-neutron separation energy in the Borromean ${ }^{22} \mathrm{C}$ nucleus." Physics Letters B, 697, 90 - 93, 2011.

[223] B. Acharya, C. Ji, and D. Phillips, "Implications of a matter-radius measurement for the structure of Carbon-22." Physics Letters B, 723, 196 200, 2013.

[224] B. Acharya, 2016, private communication.

[225] S. Mosby, N. Badger, T. Baumann, D. Bazin, M. Bennett, J. Brown, G. Christian, P. DeYoung, J. Finck, M. Gardner, J. Hinnefeld, E. Hook, E. Lunderberg, B. Luther, D. Meyer, M. Mosby, G. Peaslee, W. Rogers, J. Smith, J. Snyder, A. Spyrou, M. Strongman, and M. Thoennessen, "Search for ${ }^{21} \mathrm{C}$ and constraints on ${ }^{22} \mathrm{C}$." Nuclear Physics A, 909, 69 - 78, 2013.

[226] M. Yamashita, L. Tomio, and T. Frederico, "Radii in weakly-bound light halo nuclei." Nuclear Physics A, 735, 40 - 54, 2004.

[227] F. Marqués, M. Labiche, N. Orr, J. Angélique, L. Axelsson, B. Benoit, U. Bergmann, M. Borge, W. Catford, S. Chappell, N. Clarke, G. Costa, N. Curtis, A. D'Arrigo, F. de Oliveira Santos, E. de Góes Brennand, O. Dorvaux, M. Freer, B. Fulton, G. Giardina, C. Gregori, S. Grévy, D. Guillemaud-Mueller, F. Hanappe, B. Heusch, B. Jonson, C. L. Brun, S. Leenhardt, M. Lewitowicz, M. López, K. Markenroth, M. Motta, A. Mueller, T. Nilsson, A. Ninane, G. Nyman, I. Piqueras, K. Riisager, M. S. Laurent, F. Sarazin, S. Singer, O. Sorlin, and L. Stuttgé, "Two-neutron interferometry as a probe of the nuclear halo." Physics Letters B, 476, 219 - 225, 2000 .

[228] T. Moriguchi, A. Ozawa, S. Ishimoto, Y. Abe, M. Fukuda, I. Hachiuma, Y. Ishibashi, Y. Ito, T. Kuboki, M. Lantz, D. Nagae, K. Namihira, D. Nishimura, T. Ohtsubo, H. Ooishi, T. Suda, H. Suzuki, T. Suzuki, M. Takechi, K. Tanaka, and T. Yamaguchi, "Density distributions of 
${ }^{11} \mathrm{Li}$ deduced from reaction cross-section measurements." Phys. Rev. C, 88, 024610, Aug 2013.

[229] R. S. Bloom, M.-G. Hu, T. D. Cumby, and D. S. Jin, "Tests of Universal Three-Body Physics in an Ultracold Bose-Fermi Mixture." Phys. Rev. Lett., 111, 105301, Sep 2013.

[230] M.-G. Hu, R. S. Bloom, D. S. Jin, and J. M. Goldwin, "Avalanche-mechanism loss at an atom-molecule Efimov resonance." Phys. Rev. A, 90, 013619, Jul 2014.

[231] K. Kato. Ph.D. thesis, University of Tokyo, 2015 .

[232] K. Kato, Y. Wang, J. Kobayashi, P. S. Julienne, and S. Inouye, "Isotopic shift of atom-dimer Efimov resonances in K-Rb mixtures: Critical effect of multichannel Feshbach physics." arXiv:1610.07900, 2016.

[233] Y. Wang, J. Wang, J. P. D'Incao, and C. H. Greene, "Universal Three-Body Parameter in Heteronuclear Atomic Systems." Phys. Rev. Lett., 109, 243201, Dec 2012.

[234] G. Barontini, C. Weber, F. Rabatti, J. Catani, G. Thalhammer, M. Inguscio, and F. Minardi, "Observation of Heteronuclear Atomic Efimov Resonances." Phys. Rev. Lett., 103, 043201, Jul 2009 .

[235] J. J. Zirbel, K.-K. Ni, S. Ospelkaus, J. P. D'Incao, C. E. Wieman, J. Ye, and D. S. Jin, "Collisional Stability of Fermionic Feshbach Molecules." Phys. Rev. Lett., 100, 143201, Apr 2008.

[236] L. J. Wacker, N. B. Jørgensen, D. Birkmose, N. Winter, M. Mikkelsen, J. Sherson, N. Zinner, and J. J. Arlt, "Universal Three-Body Physics in Ultracold KRb Mixtures." Phys. Rev. Lett., 117, 163201, Oct 2016.

[237] R. A. W. Maier, M. Eisele, E. Tiemann, and C. Zimmermann, "Efimov Resonance and Three-Body Parameter in a Lithium-Rubidium Mixture." Phys. Rev. Lett., 115, 043201, Jul 2015.

[238] T. Ottenstein, T. Lompe, M. Kohnen, A. Wenz, and S. Jochim, "Collisional Stability of a ThreeComponent Degenerate Fermi Gas." Phys. Rev. Lett., 101, 203202, Nov 2008.

[239] T. Lompe, T. Ottenstein, F. Serwane, K. Viering, A. Wenz, G. Zürn, and S. Jochim, "Atom-Dimer Scattering in a Three-Component Fermi Gas." Phys. Rev. Lett., 105, 103201, Sep 2010.
[240] T. Lompe, T. B. Ottenstein, F. Serwane, A. N. Wenz, G. ZÃCErn, and S. Jochim, "RadioFrequency Association of Efimov Trimers." Science, 330, 940-944, 2010.

[241] J. Huckans, J. Williams, E. Hazlett, R. Stites, and K. O'Hara, "Three-Body Recombination in a Three-State Fermi Gas with Widely Tunable Interactions." Phys. Rev. Lett., 102, 165302, Apr 2009.

[242] J. Williams, E. Hazlett, J. Huckans, R. Stites, Y. Zhang, and K. O'Hara, "Evidence for an Excited-State Efimov Trimer in a ThreeComponent Fermi Gas." Phys. Rev. Lett., 103, 130404, Sep 2009.

[243] E. Braaten, H.-W. Hammer, D. Kang, and L. Platter, "Three-Body Recombination of ${ }^{6} \mathrm{Li}$ Atoms with Large Negative Scattering Lengths." Phys. Rev. Lett., 103, 073202, Aug 2009.

[244] P. Naidon and M. Ueda, "Possible Efimov Trimer State in a Three-Hyperfine-Component Lithium6 Mixture." Phys. Rev. Lett., 103, 073203, Aug 2009.

[245] E. Braaten, H. W. Hammer, D. Kang, and L. Platter, "Efimov physics in ${ }^{6} \mathrm{Li}$ atoms." Phys. Rev. A, 81, 013605, Jan 2010.

[246] A. Wenz, T. Lompe, T. Ottenstein, F. Serwane, G. Zürn, and S. Jochim, "Universal trimer in a three-component Fermi gas." Phys. Rev. A, 80, 040702, Oct 2009.

[247] S. T. Rittenhouse, "Magnetic-field dependence and Efimov resonance broadening in ultracold three-body recombination." Phys. Rev. A, 81, 040701, Apr 2010.

[248] B. Huang, K. M. O’Hara, R. Grimm, J. M. Hutson, and D. S. Petrov, "Three-body parameter for Efimov states in ${ }^{6} \mathrm{Li} . "$ Phys. Rev. A, 90, 043636, Oct 2014.

[249] G. Zürn, T. Lompe, A. N. Wenz, S. Jochim, P. S. Julienne, and J. M. Hutson, "Precise Characterization of ${ }^{6} \mathrm{Li}$ Feshbach Resonances Using Trap-Sideband-Resolved RF Spectroscopy of Weakly Bound Molecules." Phys. Rev. Lett., 110, 135301, Mar 2013.

[250] L. W. Bruch and J. A. Tjon, "Binding of three identical bosons in two dimensions." Phys. Rev. A, 19, 425-432, Feb 1979.

[251] T. K. Lim and P. A. Maurone, "Nonexistence of the Efimov effect in two dimensions." Phys. Rev. B, 22, 1467-1469, Aug 1980. 
[252] S. Vugal'ter and G. Zhislin, "On finiteness of the discrete spectrum of the energy operators of multiatomic molecules." Theoretical and Mathematical Physics, 55, 357-365, 1983.

[253] E. H. Lieb and W. Liniger, "Exact Analysis of an Interacting Bose Gas. I. The General Solution and the Ground State." Phys. Rev., 130, 1605-1616, May 1963.

[254] J. B. McGuire, "Study of Exactly Soluble One-Dimensional N-Body Problems." Journal of Mathematical Physics, 5, 622-636, 1964.

[255] O. Kartavtsev, A. Malykh, and S. Sofianos, "Bound states and scattering lengths of three two-component particles with zero-range interactions under one-dimensional confinement." Journal of Experimental and Theoretical Physics, 108, 365-373, 2009.

[256] M. Girardeau, "Relationship between Systems of Impenetrable Bosons and Fermions in One Dimension." Journal of Mathematical Physics, 1, 516-523, 1960.

[257] Y. Nishida and D. T. Son, "Universal fourcomponent Fermi gas in one dimension." Phys. Rev. A, 82, 043606, Oct 2010.

[258] H.-W. Hammer and D. T. Son, "Universal Properties of Two-Dimensional Boson Droplets." Phys. Rev. Lett., 93, 250408, Dec 2004.

[259] O. I. Kartavtsev and A. V. Malykh, "Universal low-energy properties of three two-dimensional bosons." Phys. Rev. A, 74, 042506, Oct 2006.

[260] L. Platter, H.-W. Hammer, and U.-G. Meißner, "Universal Properties of the Four-Boson System in Two Dimensions." Few-Body Systems, 35, 169-174, 2004.

[261] L. Pricoupenko and P. Pedri, "Universal $(1+2)-$ body bound states in planar atomic waveguides." Phys. Rev. A, 82, 033625, Sep 2010.

[262] F. F. Bellotti, T. Frederico, M. T. Yamashita, D. V. Fedorov, A. S. Jensen, and N. T. Zinner, "Scaling and universality in two dimensions: three-body bound states with short-ranged interactions." Journal of Physics B: Atomic, Molecular and Optical Physics, 44, 205302, 2011.

[263] I. Bloch, "Ultracold quantum gases in optical lattices." Nature Physics, 1, 23-30, Oct 2005.

[264] J. Levinsen, P. Massignan, and M. M. Parish, "Efimov Trimers under Strong Confinement." Phys. Rev. X, 4, 031020, Jul 2014.
[265] D. S. Petrov and G. V. Shlyapnikov, "Interatomic collisions in a tightly confined Bose gas." Phys. Rev. A, 64, 012706, Jun 2001.

[266] J. Levinsen and M. M. Parish, "Bound States in a Quasi-Two-Dimensional Fermi Gas." Phys. Rev. Lett., 110, 055304, Jan 2013.

[267] M. T. Yamashita, F. F. Bellotti, T. Frederico, D. V. Fedorov, A. S. Jensen, and N. T. Zinner, "Weakly bound states of two- and three-boson systems in the crossover from two to three dimensions." Journal of Physics B: Atomic, Molecular and Optical Physics, 48, 025302, 2015.

[268] S. Lammers, I. Boettcher, and C. Wetterich, "Dimensional crossover of nonrelativistic bosons." Phys. Rev. A, 93, 063631, Jun 2016.

[269] Y. Nishida and S. Tan, "Universal Fermi Gases in Mixed Dimensions." Phys. Rev. Lett., 101, 170401, Oct 2008.

[270] Y. Nishida and S. Tan, "Liberating Efimov Physics from Three Dimensions." Few-Body Systems, 51, 191-206, 2011.

[271] Z. Nussinov and S. Nussinov, "Triviality of the BCS-BEC crossover in extended dimensions: Implications for the ground state energy." Phys. Rev. A, 74, 053622, Nov 2006.

[272] Y. Nishida and S. Tan, "Confinement-induced Efimov resonances in Fermi-Fermi mixtures." Phys. Rev. A, 79, 060701, Jun 2009.

[273] Y. Nishida, "Phases of a bilayer Fermi gas." Phys. Rev. A, 82, 011605, Jul 2010.

[274] T. Yin, P. Zhang, and W. Zhang, "Stable heteronuclear few-atom bound states in mixed dimensions." Phys. Rev. A, 84, 052727, Nov 2011.

[275] G. Lamporesi, J. Catani, G. Barontini, Y. Nishida, M. Inguscio, and F. Minardi, "Scattering in Mixed Dimensions with Ultracold Gases." Phys. Rev. Lett., 104, 153202, Apr 2010.

[276] F. Minardi, G. Barontini, J. Catani, G. Lamporesi, Y. Nishida, and M. Inguscio, "Bose-Bose mixtures in reduced dimensions." Journal of Physics: Conference Series, 264, 012016, 2011.

[277] A. Gogolin, C. Mora, and R. Egger, "Analytical Solution of the Bosonic Three-Body Problem." Phys. Rev. Lett., 100, 140404, Apr 2008.

[278] V. Flambaum, G. Gribakin, and C. Harabati, "Analytical calculation of cold-atom scattering." Phys. Rev. A, 59, 1998-2005, Mar 1999. 
[279] S. Moszkowski, S. Fleck, A. Krikeb, L. Theußl, J.-M. Richard, and K. Varga, "Binding three or four bosons without bound subsystems." Phys. Rev. A, 62, 032504, Aug 2000.

[280] J. P. D'Incao, C. H. Greene, and B. D. Esry, "The short-range three-body phase and other issues impacting the observation of Efimov physics in ultracold quantum gases." Journal of Physics B: Atomic, Molecular and Optical Physics, 42, 044016, 2009.

[281] N. Gross, Z. Shotan, S. Kokkelmans, and L. Khaykovich, "Nuclear-Spin-Independent Short-Range Three-Body Physics in Ultracold Atoms." Phys. Rev. Lett., 105, 103203, Sep 2010.

[282] R. J. Wild, P. Makotyn, J. M. Pino, E. A. Cornell, and D. S. Jin, "Measurements of Tan's Contact in an Atomic Bose-Einstein Condensate." Phys. Rev. Lett., 108, 145305, Apr 2012.

[283] S. Knoop, J. Borbely, W. Vassen, and S. Kokkelmans, "Universal three-body parameter in ultracold ${ }^{4} \mathrm{He}^{*}$." Phys. Rev. A, 86, 062705, Dec 2012.

[284] C. Chin, "Universal scaling of Efimov resonance positions in cold atom systems." arXiv:1111.1484, 2011.

[285] E. Hiyama and M. Kamimura, "Universality in Efimov-associated tetramers in ${ }^{4}$ He." Phys. Rev. A, 90, 052514, Nov 2014.

[286] D. Blume, "Efimov Physics and the Three-Body Parameter for Shallow van der Waals Potentials." Few-Body Systems, 56, 859-867, 2015.

[287] J. E. Jones, "On the Determination of Molecular Fields. II. From the Equation of State of a Gas," Proceedings of the Royal Society of London A: Mathematical, Physical and Engineering Sciences, 106, 463-477, 1924.

[288] P. Sørensen, D. Fedorov, A. Jensen, and N. Zinner, "Efimov physics and the three-body parameter within a two-channel framework." Phys. Rev. A, 86, 052516, Nov 2012.

[289] B. M. Axilrod and E. Teller, "Interaction of the van der Waals Type Between Three Atoms." The Journal of Chemical Physics, 11, 299-300, 1943.

[290] D. Ernst, C. Shakin, and R. Thaler, "Separable Representations of Two-Body Interactions." Phys. Rev. C, 8, 46-52, Jul 1973.

[291] G. F. Gribakin and V. V. Flambaum, "Calculation of the scattering length in atomic collisions using the semiclassical approximation." Phys. Rev. A, 48, 546-553, Jul 1993.
[292] B. Gao, "Quantum-defect theory of atomic collisions and molecular vibration spectra." Phys. Rev. A, 58, 4222-4225, Nov 1998.

[293] G. Pöschl and E. Teller, "Bemerkungen zur Quantenmechanik des anharmonischen Oszillators." Zeitschrift für Physik, 83, 143-151, 1933.

[294] S. Flügge, Practical Quantum Mechanics. Springer, 1999.

[295] H. Yukawa, S. Sakata, and M. Taketani, "On the Interaction of Elementary Particles. III." Progress of Theoretical Physics Supplement, 1, 24-45, 1955.

[296] P. M. Morse, "Diatomic Molecules According to the Wave Mechanics. II. Vibrational Levels." Phys. Rev., 34, 57-64, Jul 1929.

[297] Y. Horinouchi and M. Ueda, "Onset of a Limit Cycle and Universal Three-Body Parameter in Efimov Physics." Phys. Rev. Lett., 114, 025301, Jan 2015.

[298] Y. Nishida, "New Type of Crossover Physics in Three-Component Fermi Gases." Phys. Rev. Lett., 109, 240401, Dec 2012.

[299] Y. Castin and E. Tignone, "Trimers in the resonant $(2+1)$-fermion problem on a narrow Feshbach resonance: Crossover from Efimovian to hydrogenoid spectrum." Phys. Rev. A, 84, 062704, Dec 2011.

[300] S. Endo and Y. Castin, "Unitary bosonboson and boson-fermion mixtures: third virial coefficient and three-body parameter on a narrow Feshbach resonance." The European Physical Journal D, 70, 238, 2016.

[301] R. Schmidt, S. P. Rath, and W. Zwerger, "Efimov physics beyond universality." Eur. Phys. J. B, 85, 386, 2012.

[302] Y. Wang and P. S. Julienne, "Universal van der Waals physics for three cold atoms near Feshbach resonances," Nature Physics, 10, 768-773, Aug 2014.

[303] B. Gao, E. Tiesinga, C. J. Williams, and P. S. Julienne, "Multichannel quantum-defect theory for slow atomic collisions." Phys. Rev. A, 72, 042719, Oct 2005.

[304] B. Gao, "Analytic description of atomic interaction at ultracold temperatures. II. Scattering around a magnetic Feshbach resonance." Phys. Rev. A, 84, 022706, Aug 2011.

[305] R. D. Amado and F. C. Greenwood, "There Is No Efimov Effect for Four or More Particles." Phys. Rev. D, 7, 2517-2519, Apr 1973. 
[306] A. Deltuva, "Universal Four-Boson System: Dimer-Atom-Atom Efimov Effect and Recombination Reactions." Few-Body Systems, 54, 1517-1521, 2013.

[307] J. Tjon, "Bound states of ${ }^{4} \mathrm{He}$ with local interactions." Physics Letters B, 56, 217 - 220, 1975 .

[308] J. Tjon, "The three and four nucleon systems (theory)." Nuclear Physics A, 353, 47 - 60, 1981.

[309] S. K. Adhikari, T. Frederico, and I. D. Goldman, "Perturbative Renormalization in Quantum FewBody Problems." Phys. Rev. Lett., 74, 487-491, Jan 1995.

[310] M. T. Yamashita, L. Tomio, A. Delfino, and T. Frederico, "Four-boson scale near a Feshbach resonance." Europhys. Lett., 75, 555, 2006.

[311] M. R. Hadizadeh, M. T. Yamashita, L. Tomio, A. Delfino, and T. Frederico, "Scaling Properties of Universal Tetramers." Phys. Rev. Lett., 107, 135304, Sep 2011.

[312] L. Platter, H.-W. Hammer, and U.-G. Meißner, "Four-boson system with short-range interactions." Phys. Rev. A, 70, 052101, Nov 2004.

[313] O. Yakubovsky, "On the integral equations in the theory of N particle scattering," Sov. J. Nucl. Phys., 5, 937, 1967.

[314] A. Deltuva, "Efimov physics in bosonic atomtrimer scattering." Phys. Rev. A, 82, 040701, Oct 2010.

[315] A. Deltuva, "Efimov Physics in Four-Boson Scattering." Few-Body Systems, 50, 391-394, 2010.

[316] A. Deltuva, "Shallow Efimov tetramer as inelastic virtual state and resonant enhancement of the atom-trimer relaxation," EPL (Europhysics Letters), 95, 43002, 2011.

[317] A. Deltuva, "Universality in bosonic dimer-dimer scattering." Phys. Rev. A, 84, 022703, Aug 2011.

[318] A. Deltuva, R. Lazauskas, and L. Platter, "Universality in Four-Body Scattering." FewBody Systems, 51, 235-247, 2011.

[319] A. Deltuva, "Dimer-atom-atom Recombination in the Universal Four-boson System." Few-Body Systems, 54, 2419-2425, 2012.

[320] A. Deltuva, "Momentum-space calculation of four-boson recombination." Phys. Rev. A, 85, 012708, Jan 2012.
[321] A. Deltuva, "Universal bosonic tetramers of dimer-atom-atom structure." Phys. Rev. A, 85, 042705, Apr 2012.

[322] A. Deltuva, "Properties of Universal Bosonic Tetramers." Few-Body Systems, 54, 569-577, 2012.

[323] P. Grassberger and W. Sandhas, "Systematical treatment of the non-relativistic n-particle scattering problem." Nuclear Physics B, 2, 181 - 206, 1967.

[324] E. O. Alt, P. Grassberger, and W. Sandhas, "Systematical and practical treatment of the fewbody problem," JINR Report, E46688, 1, 1972.

[325] Y. Wang, W. B. Laing, J. von Stecher, and B. D. Esry, "Efimov Physics in Heteronuclear Four-Body Systems." Phys. Rev. Lett., 108, 073201 , Feb 2012.

[326] D. Blume and Y. Yan, "Generalized Efimov Scenario for Heavy-Light Mixtures." Phys. Rev. Lett., 113, 213201, Nov 2014.

[327] H. W. L. Naus and J. A. Tjon, "The Efimov effect in a four-body system." Few-Body Systems, 2, 121-126, 1987.

[328] J. von Stecher, "Weakly bound cluster states of Efimov character." Journal of Physics B: Atomic, Molecular and Optical Physics, 43, $101002,2010$.

[329] J. von Stecher, "Five- and Six-Body Resonances Tied to an Efimov Trimer." Phys. Rev. Lett., 107, 200402, Nov 2011.

[330] J. Horne, J. A. Salas, and K. Varga, "Energy and Structure of Few-Boson Systems." Few-Body Systems, 55, 1245-1252, 2014.

[331] M. Gattobigio and A. Kievsky, "Universality and scaling in the $N$-body sector of Efimov physics." Phys. Rev. A, 90, 012502, Jul 2014.

[332] Y. Yan and D. Blume, "Energy and structural properties of $N$-boson clusters attached to three-body Efimov states: Two-body zero-range interactions and the role of the three-body regulator." Phys. Rev. A, 92, 033626, Sep 2015.

[333] Y. Yan and D. Blume, "Temperature dependence of small harmonically trapped atom systems with Bose, Fermi, and Boltzmann statistics." Phys. Rev. A, 90, 013620, Jul 2014.

[334] A. N. Nicholson, "N-Body Efimov States from Two-Particle Noise." Phys. Rev. Lett., 109, 073003, Aug 2012. 
[335] A. Zenesini, B. Huang, M. Berninger, S. Besler, H.-C. Nägerl, F. Ferlaino, R. Grimm, C. H. Greene, and J. von Stecher, "Resonant five-body recombination in an ultracold gas of bosonic atoms." New Journal of Physics, 15, 043040, 2013.

[336] D. S. Petrov, C. Salomon, and G. V. Shlyapnikov, "Scattering properties of weakly bound dimers of fermionic atoms." Phys. Rev. A, 71, 012708, Jan 2005.

[337] D. S. Petrov, C. Salomon, and G. V. Shlyapnikov, "Weakly Bound Dimers of Fermionic Atoms." Phys. Rev. Lett., 93, 090404, Aug 2004.

[338] A. J. Leggett, "Diatomic molecules and Cooper pairs," in Modern trends in the theory of condensed matter. Springer, 1980, 13-27.

[339] P. Nozieres and S. Schmitt-Rink, "Bose condensation in an attractive fermion gas: From weak to strong coupling superconductivity," Journal of Low Temperature Physics, 59, 195-211, 1985.

[340] T. Bourdel, L. Khaykovich, J. Cubizolles, J. Zhang, F. Chevy, M. Teichmann, L. Tarruell, S. J. J. M. F. Kokkelmans, and C. Salomon, "Experimental Study of the BEC-BCS Crossover Region in Lithium 6." Phys. Rev. Lett., 93, 050401, Jul 2004.

[341] C. A. R. Sá de Melo, M. Randeria, and J. R. Engelbrecht, "Crossover from BCS to Bose superconductivity: Transition temperature and time-dependent Ginzburg-Landau theory." Phys. Rev. Lett., 71, 3202-3205, Nov 1993.

[342] R. Haussmann, "Crossover from BCS superconductivity to Bose-Einstein condensation: A selfconsistent theory," Zeitschrift für Physik B Condensed Matter, 91, 291-308, 1993.

[343] Y. Ohashi, "Effective interaction between molecules in the strong-coupling BEC regime of a superfluid Fermi gas," Journal of the Physical Society of Japan, 74, 2659-2662, 2005.

[344] S. Diehl, S. Floerchinger, H. Gies, J. Pawlowkski, and C. Wetterich, "Functional renormalization group approach to the BCS-BEC crossover," Annalen der Physik, 522, 615-656, 2010.

[345] J. Levinsen and V. Gurarie, "Properties of strongly paired fermionic condensates." Phys. Rev. A, 73, 053607, May 2006.

[346] P. Pieri and G. C. Strinati, "Strong-coupling limit in the evolution from BCS superconductivity to Bose-Einstein condensation." Phys. Rev. B, 61, 15 370-15 381, Jun 2000.
[347] M. C. Birse, B. Krippa, and N. R. Walet, "Convergence of a renormalization-group approach to dimer-dimer scattering." Phys. Rev. A, 83, 023621, Feb 2011.

[348] I. V. Brodsky, M. Y. Kagan, A. V. Klaptsov, R. Combescot, and X. Leyronas, "Exact diagrammatic approach for dimer-dimer scattering and bound states of three and four resonantly interacting particles." Phys. Rev. A, 73, 032724, Mar 2006.

[349] J. P. D'Incao, S. T. Rittenhouse, N. P. Mehta, and C. H. Greene, "Dimer-dimer collisions at finite energies in two-component Fermi gases." Phys. Rev. A, 79, 030501, Mar 2009.

[350] D. S. Petrov, C. Salomon, and G. V. Shlyapnikov, "Diatomic molecules in ultracold Fermi gases novel composite bosons." Journal of Physics B: Atomic, Molecular and Optical Physics, 38, S645, 2005.

[351] M. Iskin and C. A. R. Sá de Melo, "Fermi-Fermi mixtures in the strong-attraction limit." Phys. Rev. A, 77, 013625, Jan 2008.

[352] F. Alzetto, R. Combescot, and X. Leyronas, "Dimer-dimer scattering length for fermions with different masses: Analytical study for large mass ratio." Phys. Rev. A, 87, 022704, Feb 2013.

[353] D. Blume, "Universal Four-Body States in Heavy-Light Mixtures with a Positive Scattering Length." Phys. Rev. Lett., 109, 230404, Dec 2012 .

[354] S. Endo and Y. Castin, "Absence of a four-body Efimov effect in the $2+2$ fermionic problem." Phys. Rev. A, 92, 053624, Nov 2015.

[355] D. Blume and K. M. Daily, "Breakdown of Universality for Unequal-Mass Fermi Gases with Infinite Scattering Length." Phys. Rev. Lett., 105, 170403, Oct 2010.

[356] A. Michelangeli and P. Pfeiffer, "Stability of the $(2+2)$-fermionic system with zero-range interaction." Journal of Physics A: Mathematical and Theoretical, 49, 105301, 2016.

[357] B. Bazak and D. S. Petrov, "Five-Body Efimov Effect and Universal Pentamer in Fermionic Mixtures." Phys. Rev. Lett., 118, 083002, Feb 2017.

[358] D. Blume and K. M. Daily, "Few-body resonances of unequal-mass systems with infinite interspecies two-body $s$-wave scattering length." Phys. Rev. A, 82, 063612, Dec 2010. 
[359] D. J. MacNeill and F. Zhou, "Pauli Blocking Effect on Efimov States near a Feshbach Resonance." Phys. Rev. Lett., 106, 145301, Apr 2011.

[360] P. Niemann and H.-W. Hammer, "Pauli-blocking effects and Cooper triples in three-component Fermi gases." Phys. Rev. A, 86, 013628, Jul 2012.

[361] N. G. Nygaard and N. T. Zinner, "Efimov three-body states on top of a Fermi sea." New Journal of Physics, 16, 023026, 2014.

[362] L. N. Cooper, "Bound Electron Pairs in a Degenerate Fermi Gas." Phys. Rev., 104, 1189-1190, Nov 1956.

[363] N. G. Zinner, N. T.and Nygaard, "Efimov States in Li-Cs Mixtures within a Minimal Model." Few-Body Systems, 56, 125-131, 2015.

[364] C. J. M. Mathy, M. M. Parish, and D. A. Huse, "Trimers, Molecules, and Polarons in Mass-Imbalanced Atomic Fermi Gases." Phys. Rev. Lett., 106, 166404, Apr 2011.

[365] N. T. Zinner, "Efimov states of heavy impurities in a Bose-Einstein condensate." EPL (Europhysics Letters), 101, 60009, 2013.

[366] N. Bogolubov, "On the theory of superfluidity," Journal of Physics (USSR), 11, 23, 1947.

[367] C. J. Pethick and H. Smith, Bose-Einstein Condensation in Dilute Gases. . Cambridge University Press, 2002.

[368] N. B. Jørgensen, L. Wacker, K. T. Skalmstang, M. M. Parish, J. Levinsen, R. S. Christensen, G. M. Bruun, and J. J. Arlt, "Observation of Attractive and Repulsive Polarons in a BoseEinstein Condensate." Phys. Rev. Lett., 117, 055302, Jul 2016.

[369] M.-G. Hu, M. J. Van de Graaff, D. Kedar, J. P. Corson, E. A. Cornell, and D. S. Jin, "Bose Polarons in the Strongly Interacting Regime." Phys. Rev. Lett., 117, 055301, Jul 2016.

[370] J. Levinsen, M. M. Parish, and G. M. Bruun, "Impurity in a Bose-Einstein Condensate and the Efimov Effect." Phys. Rev. Lett., 115, 125302, Sep 2015.

[371] L. A. P. Ardila and S. Giorgini, "Impurity in a Bose-Einstein condensate: Study of the attractive and repulsive branch using quantum Monte Carlo methods." Phys. Rev. A, 92, 033612, Sep 2015.
[372] S. Cowell, H. Heiselberg, I. E. Mazets, J. Morales, V. R. Pandharipande, and C. J. Pethick, "Cold Bose Gases with Large Scattering Lengths." Phys. Rev. Lett., 88, 210403, May 2002.

[373] T.-L. Ho, "Universal Thermodynamics of Degenerate Quantum Gases in the Unitarity Limit." Phys. Rev. Lett., 92, 090402, Mar 2004.

[374] Y.-L. Lee and Y.-W. Lee, "Universality and stability for a dilute Bose gas with a Feshbach resonance." Phys. Rev. A, 81, 063613, Jun 2010.

[375] J. M. Diederix, T. C. F. van Heijst, and H. T. C. Stoof, "Ground state of a resonantly interacting Bose gas." Phys. Rev. A, 84, 033618, Sep 2011.

[376] W. Li and T.-L. Ho, "Bose Gases near Unitarity." Phys. Rev. Lett., 108, 195301, May 2012.

[377] D. Borzov, M. S. Mashayekhi, S. Zhang, J.-L. Song, and F. Zhou, "Three-dimensional Bose gas near a Feshbach resonance." Phys. Rev. A, 85, 023620, Feb 2012.

[378] S.-J. Jiang, W.-M. Liu, G. W. Semenoff, and F. Zhou, "Universal Bose gases near resonance: A rigorous solution." Phys. Rev. A, 89, 033614, Mar 2014.

[379] S. Piatecki and K. Werner, "Efimov-driven phase transitions of the unitary Bose gas." Nat Commun, 5, 3503, March 2014.

[380] R. J. Fletcher, A. L. Gaunt, N. Navon, R. P. Smith, and Z. Hadzibabic, "Stability of a Unitary Bose Gas." Phys. Rev. Lett., 111, 125303, Sep 2013.

[381] P. Makotyn, C. E. Klauss, D. L. Goldberger, E. A. Cornell, and D. S. Jin, "Universal dynamics of a degenerate unitary Bose gas." Nat. Phys., 10, 116-119, Feb 2014.

[382] S. Tan, "Energetics of a strongly correlated Fermi gas." Annals of Physics, 323, 2952 - 2970, 2008.

[383] S. Tan, "Large momentum part of a strongly correlated Fermi gas." Annals of Physics, 323, $2971-2986,2008$.

[384] S. Tan, "Generalized virial theorem and pressure relation for a strongly correlated Fermi gas." Annals of Physics, 323, 2987 - 2990, 2008.

[385] R. Combescot, F. Alzetto, and X. Leyronas, "Particle distribution tail and related energy formula." Phys. Rev. A, 79, 053640, May 2009.

[386] Y. Castin and F. Werner, "Single-particle momentum distribution of an Efimov trimer." Phys. Rev. A, 83, 063614, Jun 2011. 
[387] A. M. J. Schakel, "Tan Relations in Dilute Bose Gases." arXiv:1007.3452, 2010.

[388] E. Braaten, D. Kang, and L. Platter, "Universal Relations for Identical Bosons from Three-Body Physics." Phys. Rev. Lett., 106, 153005, Apr 2011.

[389] D. H. Smith, E. Braaten, D. Kang, and L. Platter, "Two-Body and Three-Body Contacts for Identical Bosons near Unitarity." Phys. Rev. Lett., 112, 110402, Mar 2014.

[390] A. G. Sykes, J. P. Corson, J. P. D'Incao, A. P. Koller, C. H. Greene, A. M. Rey, K. R. A. Hazzard, and J. L. Bohn, "Quenching to unitarity: Quantum dynamics in a threedimensional Bose gas." Phys. Rev. A, 89, 021601, Feb 2014.

[391] M. Rossi, L. Salasnich, F. Ancilotto, and F. Toigo, "Monte Carlo simulations of the unitary Bose gas." Phys. Rev. A, 89, 041602, Apr 2014.

[392] T. Comparin and W. Krauth, "Momentum Distribution in the Unitary Bose Gas from First Principles." Phys. Rev. Lett., 117, 225301, Nov 2016.

[393] A. Pais and G. E. Uhlenbeck, "On the Quantum Theory of the Third Virial Coefficient." Phys. Rev., 116, 250-269, Oct 1959.
[394] Y. Castin and F. Werner, "Le troisième coefficient du viriel du gaz de Bose unitaire." Canadian Journal of Physics, 91, 382-389, 2013.

[395] C. Gao, S. Endo, and Y. Castin, "The third virial coefficient of a two-component unitary Fermi gas across an Efimov-effect threshold." EPL (Europhysics Letters), 109, 16003, 2015.

[396] M. Barth and J. Hofmann, "Efimov correlations in strongly interacting Bose gases." Phys. Rev. A, 92, 062716, Dec 2015.

[397] P. F. Bedaque and J. P. D'Incao, "Superfluid phases of the three-species fermion gas." Annals of Physics, 324, 1763 - 1768, 2009.

[398] S. Endo, A. M. García-García, and P. Naidon, "Universal clusters as building blocks of stable quantum matter." Phys. Rev. A, 93, 053611, May 2016.

[399] P. Naidon, S. Endo, and A. M. García-García, "Scattering of universal fermionic clusters in the resonating group method." Journal of Physics B: Atomic, Molecular and Optical Physics, 49, $034002,2016$.

[400] M. Jona-Lasinio and L. Pricoupenko, "Three Resonant Ultracold Bosons: Off-Resonance Effects." Phys. Rev. Lett., 104, 023201, Jan 2010.

\section{List of changes}

- Introduction: A short history of Efimov physics: the word "length" was added after "van der Waals".

- Section "4.5.2 Structure" [Section 2.1.5.2 in the published version], end of third paragraph: the following incorrect sentence was removed: "For the full wave function of equation (2.16), one obtains the average value of $\alpha$ to be close to $\pi / 6$, corresponding to an elongated triangle". See https://doi.org/10.1103/PhysRevA.104.059903.

- Section "4.7.1 Helium-4" [Section 2.1.7.1 in the published version]: "The range of this potential can be characterised by its van der Waals length, which is about $0.54 \mathrm{~nm}$ ", the value was changed to $0.27 \mathrm{~nm}$ to be consistent with the definition given in the van der Waals universality section.

- Section "5.2 The super-Efimov effect", after Eq. (5.5) [Section 2.2.2 after Eq. (2.57) in the published version]: the symbol log was replaced by $\ln$.

- Figure 11.3 [Figure 22 in the published version]: the top panel was corrected, due to a wrongly calculted curve in the original figure. See https://doi.org/10.1103/PhysRevA.104.059903.

- Figure 11.3 [Figure 22 in the published version]: the words "for a right angle between the Jacobi vectors" were added to the caption.

- Formula after Eq. (11.42) [Eq. (4.45) on page 54 in the published version]: a minus sign was added before the factor $2 \pi$ in the exponential and absolute value bars were added to $E(n)$. 